\title{
Photoinduced Nickel-Catalyzed Deaminative Cross-Electrophile Coupling
}

\author{
Tao Yang, ${ }^{\ddagger},[a]$ Yi Wei, ${ }^{\ddagger},[a]$ and Ming Joo Koh*[a]
[a] Prof. Dr. M. J. Koh
Department of Chemistry, National University of Singapore,
4 Science Drive 2, Republic of Singapore, 117544
*E-mail: chmkmj@nus.edu.sg
[†] These authors contributed equally.

\begin{abstract}
The construction of carbon-carbon bonds through crosscoupling between two electrophiles in the absence of excess metallic reducing agents is a desirable objective in chemistry. Here, we show that $\mathrm{N}$-alkylpyridinium salts can be efficiently merged with aryl or alkyl halides in an intermolecular fashion, affording products in up to $90 \%$ yield at ambient temperature. These reactions harness the ability of $\mathrm{N}$-alkylpyridinium salts to form electron donor-acceptor complexes with Hantzsch esters, enabling photoinduced single-electron transfer and fragmentation to afford alkyl radicals that are subsequently trapped by a Ni-based catalytic species to promote $\mathrm{C}\left(\mathrm{sp}^{2}\right)-\mathrm{C}\left(\mathrm{sp}^{3}\right)$ and $\mathrm{C}\left(\mathrm{sp}^{3}\right)-\mathrm{C}\left(\mathrm{sp}^{3}\right)$ bond formation. The operationally simple protocol is applicable to site-selective cross-coupling and tolerates diverse functional groups, including those that are sensitive towards metal reductants.
\end{abstract}

Transition metal-catalyzed C-C bond forming cross-coupling ${ }^{[1]}$ has revolutionized the way countless organic molecules are assembled, and is an indispensable tool in the modern synthetic chemist's arsenal. ${ }^{[2]}$ In particular, reductive cross-electrophile coupling has witnessed rapid development over the last two decades. ${ }^{[3]}$ These transformations obviate the need to presynthesize organometallic reagents, allowing the direct union of two relatively more stable electrophilic organohalides, ${ }^{[4]}$ pseudohalides $^{[5]}$ or redox-active substrates ${ }^{[6]}$ to deliver the product.

Owing to the ubiquity, low cost and ease of accessibility associated with aliphatic primary amines, recent advances have taken advantage of these feedstock chemicals to prepare air- and moisture-stable Katritzky pyridinium salts ${ }^{[7]}$ as alkylating precursors in reductive coupling ${ }^{[8]}$ (Scheme 1A). However, examples of these deaminative processes are limited and often involve elevated temperatures ${ }^{[8 \mathrm{a}-\mathrm{d}]}$, and superstoichiometric amounts of an expensive organic reagent (such as tetrakis(dimethylamino)ethylene) ${ }^{[9]}$, diboryl compound/base ${ }^{[10]}$ or a metal (such as $\mathrm{Mn}, \mathrm{Zn})^{[\mathrm{Ba}-\mathrm{d}]}$ are required as terminal reducing agents. Consequently, problems such as reaction reproducibility that may arise from inconsistencies in the quality of commercially available metallic reductants ${ }^{[4 a, 4 c, 11]}$, as well as environmental ${ }^{[12]}$ and toxicity ${ }^{[13]}$ issues during metal waste disposal, especially when the reactions are performed at large scale are inevitable. Adding to these challenges is the incompatibility of certain reactive functional groups to metal reductive conditions (see below for further discussion). It merits mention that methods for deaminative alkylation ${ }^{[\mathrm{bb}, 14]}$ are scarce, and the majority of these $\mathrm{C}\left(\mathrm{sp}^{3}\right)-\mathrm{C}\left(\mathrm{sp}^{3}\right)$ bond-forming processes rely on olefins as alkyl group precursors.

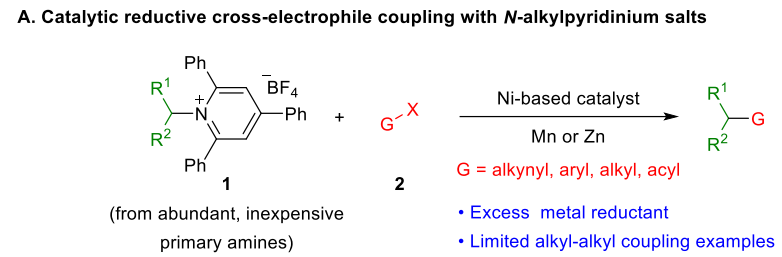

B. Catalyst-free photoinduced deaminative functionalizations via EDA complexes

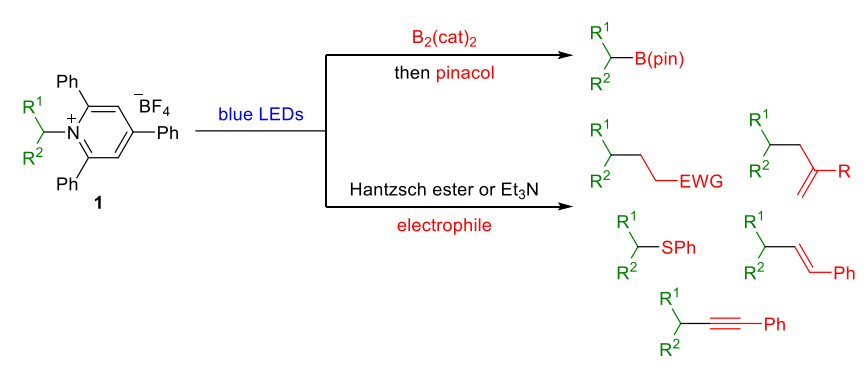

C. This work: Photoinduced Ni-catalyzed deaminative arylation and alkylation
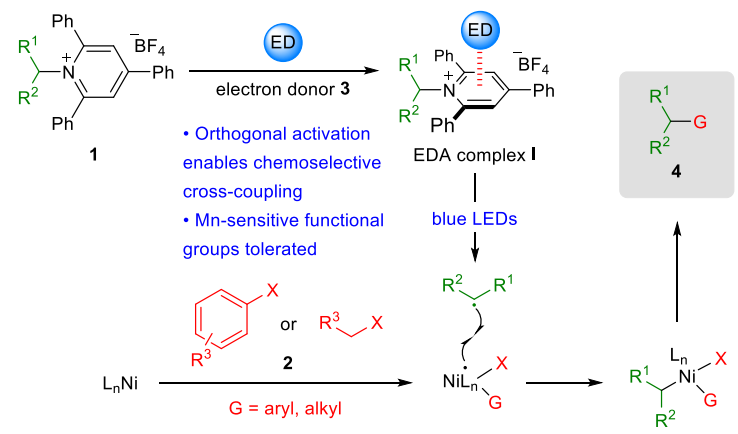

Scheme 1. Strategies for deaminative functionalizations with $\mathrm{N}$-alkylpyridinium salts.

In addition to metal-catalyzed cross-coupling ${ }^{[7 \mathrm{~d}, 14 \mathrm{~b}, 15]}$ and photocatalyzed $\mathrm{C}-\mathrm{C}$ bond formation ${ }^{[14 \mathrm{~d}-\mathrm{e}, 16]}$, the redox-active nature of $N$-alkylpyridinium salts was recently exploited in efficient photoexcitation of electron-donor-acceptor (EDA) complexes generated from the pyridinium salt with bis-(catecholato)diboron $\left(\mathrm{B}_{2} \mathrm{Cat}_{2}\right)^{[17]}$, Hantzsch ester ${ }^{[18]}$ or triethylamine ${ }^{[18]}$ to deliver $\mathrm{C}-\mathrm{X}(\mathrm{X}$ $=\mathrm{B}, \mathrm{C}, \mathrm{S})$ bonds under catalyst-free conditions (Scheme 1B). Inspired by these preceding observations, we speculated if we could leverage the photoactive EDA complex I formed between an $\mathrm{N}$-alkylpyridinium salt $\mathbf{1}$ and a relatively inexpensive electron donor 3, which under visible light irradiation would furnish an unactivated alkyl radical species that could be captured in a nickel catalytic cycle (involving the reaction of organohalide 2) to deliver the final adduct 4 (Scheme 1C). Through this new catalytic platform, stoichiometric metals or expensive reducing agents are avoided, enabling facile entry to a broader spectrum of multifunctional products that are otherwise inaccessible using 
previously established reductive regimes. Furthermore, the orthogonal activation of $\mathbf{1}$ and $\mathbf{2}$ means that difficult alkyl-alkyl cross-coupling reactions that are often susceptible to competitive homocoupling ${ }^{[4 b, 19]}$ side pathways can be achieved under mild base-free conditions at ambient temperature without an exorbitantly large excess of either reactant, thereby reducing waste generation. Herein, we describe the successful implementation of a photoinduced deaminative strategy to facilitate $\mathrm{C}\left(\mathrm{sp}^{2}\right)-\mathrm{C}\left(\mathrm{sp}^{3}\right)$ and $\mathrm{C}\left(\mathrm{sp}^{3}\right)-\mathrm{C}\left(\mathrm{sp}^{3}\right)$ cross-electrophile coupling with readily available organohalides.

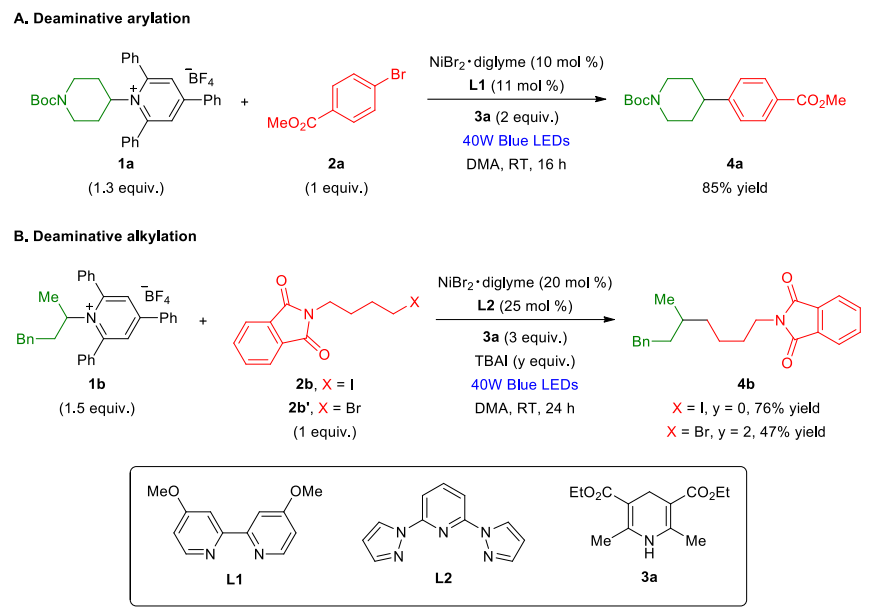

Scheme 2. Reaction conditions identified for photoinduced $\mathrm{Ni}$-catalyzed deaminative arylation and alkylation. Yields are for isolated and purified products.

A survey of various Ni-based salts, ligands, electron donors and solvents was carried out to merge pyridinium salt 1a with aryl bromide 2a under blue light irradiation at ambient temperature (see Supporting Information (SI) for details). In the event, we found that the desired deaminative arylation product $\mathbf{4 a}$ was obtained in $85 \%$ isolated yield within 16 hours, using $10 \mathrm{~mol} \%$ of $\mathrm{NiBr}_{2}$.diglyme and $11 \mathrm{~mol} \%$ of bipyridine $\mathbf{L 1}$ in the presence of Hantzsch ester $\mathbf{3} \mathbf{a}$ as reductant and DMA as solvent (Scheme 2A). Replacing the Ni complex, ligand or solvent with other aternatives led to lower yields, and no product was detected in the absence of the catalyst, $\mathbf{3 a}$ or light (see SI for details).

Reaction conditions were separately evaluated for the more challenging union of pyridinium salt $\mathbf{1} \mathbf{b}$ with alkyl iodide $\mathbf{2} \mathbf{b}$ (see $\mathrm{SI}$ for details), and $20 \mathrm{~mol} \%$ of the Ni-based complex derived from
$\mathrm{NiBr}_{2}$. diglyme and $\mathbf{L} 2$ was revealed to be optimal to furnish $\mathbf{4 b}$ in $76 \%$ isolated yield ( $83 \%$ GC yield; Scheme $2 \mathrm{~B})$. Decreasing the catalyst loading to $10 \mathrm{~mol} \%$ resulted in diminished efficiency (62\% GC yield). Remarkably, performing the alkylation using a $\mathbf{1 : 1}$ ratio of $\mathbf{1} \mathbf{b}$ and $\mathbf{2} \mathbf{b}$ delivered $\mathbf{4} \mathbf{b}$ with a respectable GC yield of $55 \%$ (see SI for details). This compares favorably with other reductive cross-electrophile protocols that employ superstoichiometric quantities of one cross-partner (2-3 equiv.) to suppress adventitious homocoupling ${ }^{[8 \mathrm{c}]}$, or entail strongly basic ${ }^{[7 \mathrm{~d} \text {, }}$ 14b] and cryogenic conditions. ${ }^{[5 \mathrm{~h}]}$ It is worth noting that the analogous alkylation with bromoalkane $\mathbf{2} \mathbf{b}$ ' in the presence of tetrabutylammonium iodide (TBAI) as additive could also generate $4 \mathrm{~b}$ in $47 \%$ yield.

With the established conditions in hand, we first examined the scope of deaminative arylation using a diverse array of pyridinium salts 1 (secondary and primary alkyl, cyclic and acyclic) as well as bromoarenes 2 (Scheme 3). In general, various electronically modified aryl bromides served as effective substrates in crosscoupling, affording products in up to $90 \%$ yield. These include molecules containing a nitrile (4c), an alcohol (4z), an aldehyde $(\mathbf{4 d})$, a ketone $(\mathbf{4 e}, \mathbf{4 j})$, an ester $(\mathbf{4 i}, \mathbf{4 k}, \mathbf{4 0}, \mathbf{4 s})$, an amide $(\mathbf{4 h}, \mathbf{4 q}$, $\mathbf{4 v - 4 z )}$, a sulfone (4f) and a sulfoamide (4g, $\mathbf{4 a c}-\mathbf{4 a d})$. Synthesis of Lewis basic heterocycle $\mathbf{4 r}$ as well as products derived from multifunctional bioactive compounds (4I-4n, 4aa-4ab, 4ae-4af) further underscores the exceptional functional group compatibility of the photoinduced catalytic reaction system.

Reactions with functionalized aliphatic iodides were subsequently investigated (Scheme 4). Deaminative alkylation was found to proceed efficiently to deliver $\mathbf{4 a g}-\mathbf{4 a t}$ in up to $73 \%$ yield. Commonly occurring functional groups such as a phthalimide (4ag, 4ai-4aj, 4ap-4aq), an amino ester (4al), an ether (4ar) as well as an electron-deficient enoate (4ah, 4as) are tolerated. Chemoselective cross-coupling with the iodoalkane motif enables access to $\mathbf{4 a k}$, which bears a reactive aryl bromide handle that could be subjected to further transformations. Similar to the processes in Scheme 3, deaminative alkylation with substrates appended to complex molecules could be effected to generate the corresponding products $4 \mathbf{a m}-\mathbf{4 a o}$. Of particular note, the formation of 4 at demonstrates a key advantage of the present photoinduced catalytic manifold, since alkyl-substituted aldehydes are found to be sensitive towards excess metallic reductants ${ }^{[\mathrm{bb}, 8 \mathrm{~d}]}$ (see SI for details). 

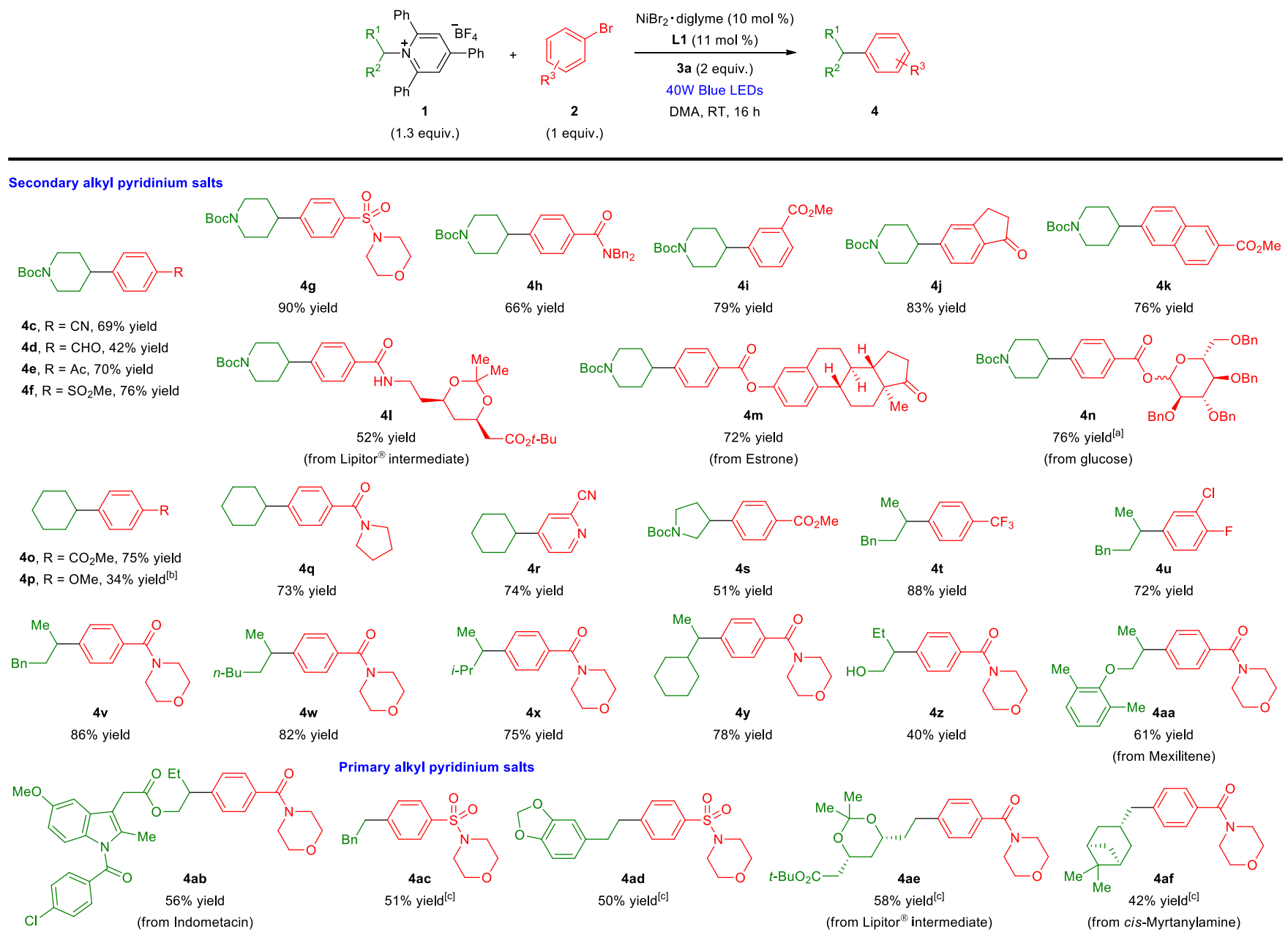

Scheme 3. The scope of deaminative arylation. Yields are for isolated and purified products. [a] $4 \mathrm{n}$ was obtained in 1:1 diastereomeric ratio. [b] The reaction was conducted with 20 mol\% Ni complex, 25 mol\% L1 and 2 equiv. pyridinium salt. [c] The reactions were conducted with 12 mol\% 4,4 '-di-t-Bu-bpy, 1.5 equiv. pyridinium salt and 3 equiv. $\mathrm{Et}_{3} \mathrm{~N}$.

(1.5 equiv.)

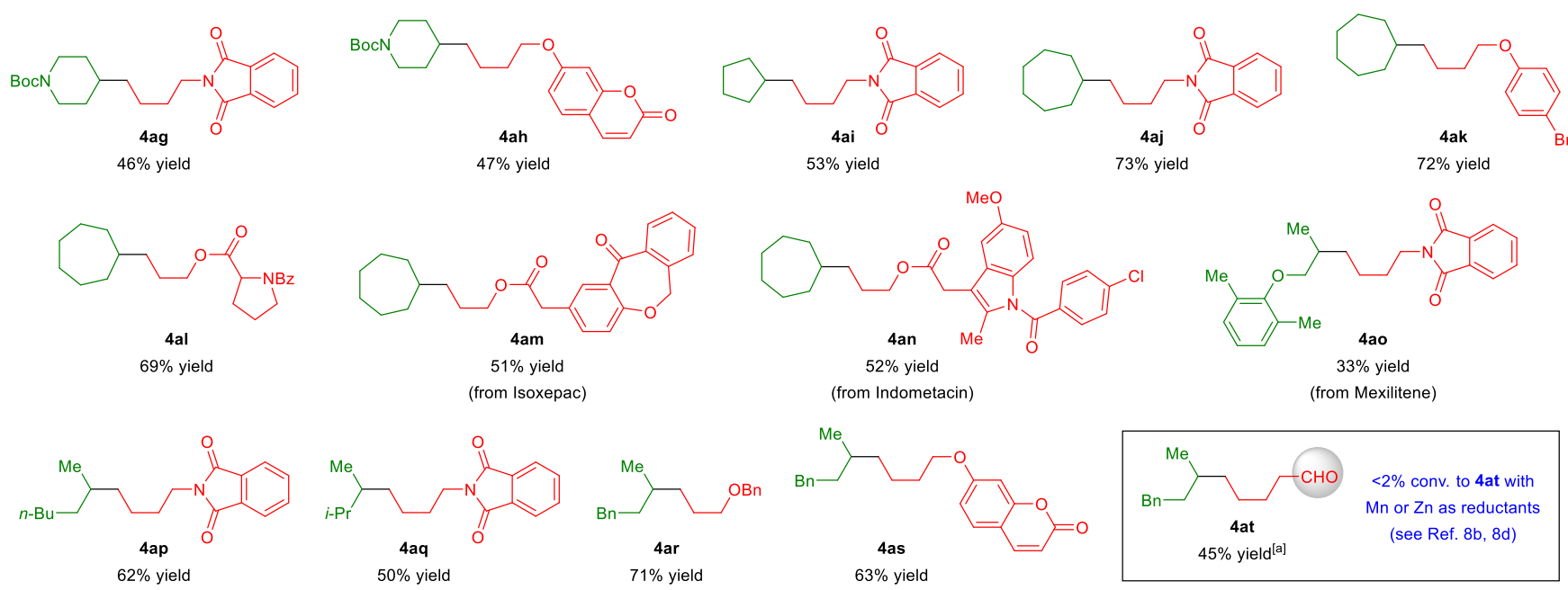

Scheme 4. The scope of deaminative alkylation. Yields are for isolated and purified products. [a] The reaction was conducted with 20 mol\% Ni complex and 25 mol\% L2. 
Formation of photoactive EDA complexes between $N$ alkylpyridinium salts $\mathbf{1}$ and $\mathbf{3 a}$ have already been documented in previous studies. ${ }^{[18]}$ Further control experiments were performed to provide evidence for the formation of radical intermediates (from 1) in our system (Scheme 5A).

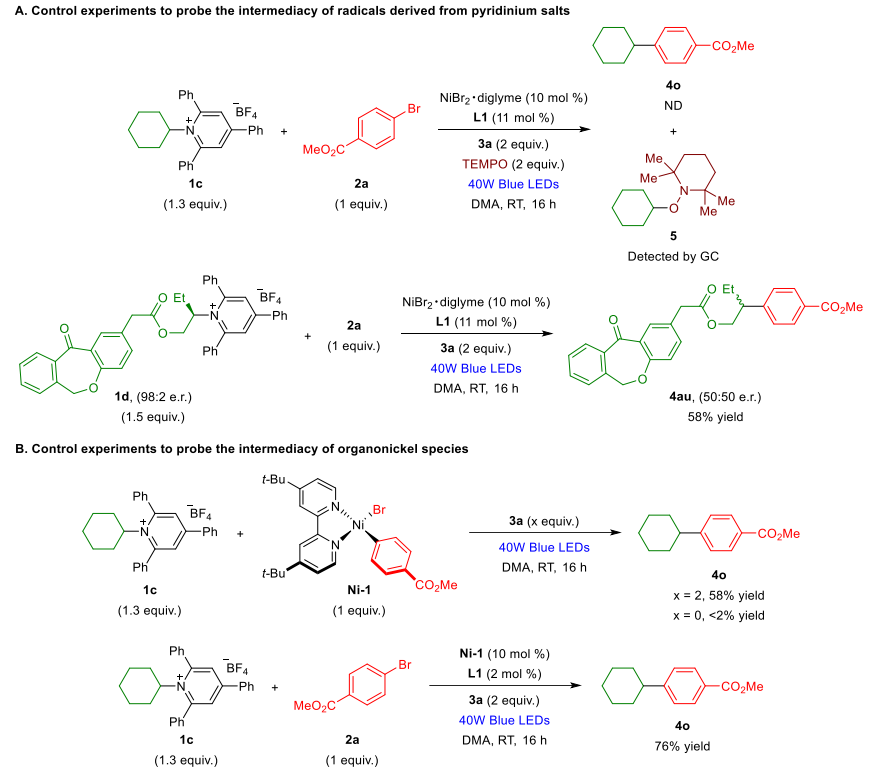

Scheme 5. Mechanistic studies.

When excess TEMPO was added to the standard reaction mixture, cross-electrophile coupling was suppressed and the TEMPO-trapped product 5 was detected by GC analysis. On the other hand, deaminative arylation of enantiomerically enriched $\mathbf{1 d}$ (prepared from commercially available $(R)$-2-amino-1-butanol) with $2 \mathrm{a}$ led to complete erosion of stereochemistry in the final product 4 au (isolated in $58 \%$ yield as a racemic mixture), suggesting the formation of a radical species through a putative single-electron transfer (SET) pathway from 1d. ${ }^{[7,8 c]}$

To probe the supposed intermediacy of organonickel species, a separately-synthesized bipyridine-Ni(II) complex $\mathrm{Ni}-1$ (derived from oxidative addition of the corresponding $\mathrm{Ni}(0)$ complex with 2a) was reacted with $\mathbf{1 c}$ under the established photoinduced cross-coupling conditions, and 40 was produced in $58 \%$ yield (Scheme 5B). As expected, $<2 \%$ conv. to 40 was observed in the absence of the electron donor $\mathbf{3 a}$. Furthermore, deaminative arylation of $\mathbf{1 c}$ with $\mathbf{2 a}$ was found to proceed smoothly in the presence of catalytic amounts of $\mathbf{N i - 1}$ and L1. These results substantiate the occurrence of an oxidative insertion process involving the organohalide $\mathbf{2}$ prior to alkylation with the pyridinium salt 1.

Based on our studies as well as past observations, we propose a tentative reaction mechanism as shown in Scheme 6. An EDA complex ${ }^{[18]}$ is likely formed between $\mathrm{N}$-alkylpyridinium salt $\mathbf{1}$ and Hantzsch ester $\mathbf{3 a}$, which then undergoes photoexcitation and SET to give dihydropyridine radical cation II and III. Subsequent deaminative fragmentation of III yields triphenylpyridine IV and alkyl radical $\mathbf{V}$, which presumably enters a $\mathrm{Ni}$ catalytic cycle by associating with $\mathrm{Ni}(\mathrm{II})$ complex ii, the oxidative insertion adduct derived from $\mathrm{Ni}(0)$ species $\mathbf{i}$ and organohalide 2 (either by direct oxidative addition or halogen atom abstraction/radical recombination $\left.{ }^{[3 a]}\right)$. The resulting alkylnickel species iii is poised to undergo reductive elimination to furnish iv with concomitant release of the desired product 4 . To turn over the cycle, we surmised that iv is reduced back to $\mathbf{i}$ in the presence of II and IV, affording protonated pyridine by-products VI and VII. Overall, the chemoselective activation of $\mathbf{1}$ and $\mathbf{2}$ enables the photoinduced catalytic cross-coupling to occur under mild ambient conditions. Further investigations to shed light on the exact nature of the in situ-generated organonickel species are ongoing and will be disclosed in due course.

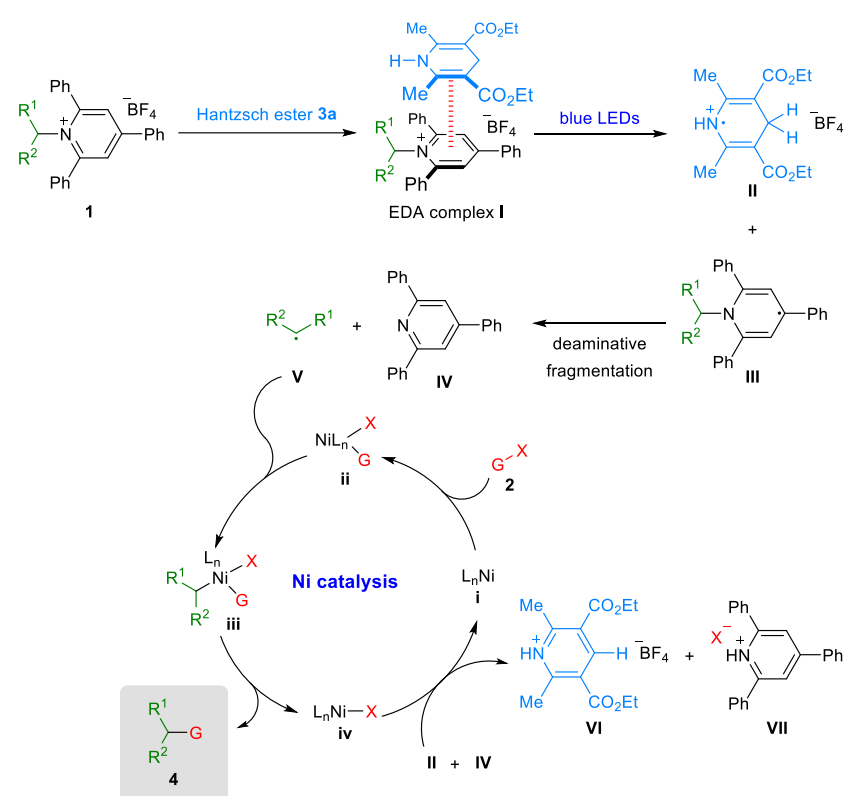

Scheme 6. Proposed mechanism for photoinduced Ni-catalyzed deaminative cross-coupling.

To showcase the value of our reaction system, dibromide $\mathbf{2 c}$ was subjected to sequences of photoinduced deaminative crosscoupling using $\mathrm{N}$-alkylpyridinium salts $\mathbf{1 b}$ and $\mathbf{1 e}$ (Scheme 7A). In the event, the alkyl bromide motif within $2 \mathrm{c}$ selectively underwent alkylation with 1e to give 6 in 55\% yield. Following another alkylation with 1b, $\mathbf{7}$ was obtained in $70 \%$ yield. In a second set of transformations, 2c was selectively alkylated at the bromoarene site using 1e to furnish 8 in $62 \%$ yield, which was further converted to 9 in $46 \%$ yield. Thus, access to both regioisomers 7 and 9 could be readily achieved without the need for tedious protection/deprotection strategies.

In stark contrast, treatment of $\mathbf{2 c}$ to previously reported reductive deaminative conditions (with $\mathrm{Mn}$ or $\mathrm{Zn}$ ) ${ }^{[8 \mathrm{bb}, 8 \mathrm{~d}]}$ only led to complex product mixtures (Scheme 7B). These results highlight the unique capability of our photoinduced catalytic regime to effectively discriminate the multiple reactive sites of a substrate, which is crucial for attaining site-selective cross-coupling. ${ }^{[20]}$

In conclusion, we have developed a new catalytic manifold for deaminative arylation and alkylation that operates under mild reaction conditions. Central to this approach is the facile generation of aliphatic radicals from photoexcitation of pyridinium salt EDA complexes, and their subsequent engagement with an appropriate organonickel species to promote carbon-carbon bond formation. The present method demonstrates excellent functional group tolerance and offers a reliable avenue for site-selective installation of carbogenic motifs through orthogonal halide activation. Further extension of the strategy to other related transformations is ongoing. 


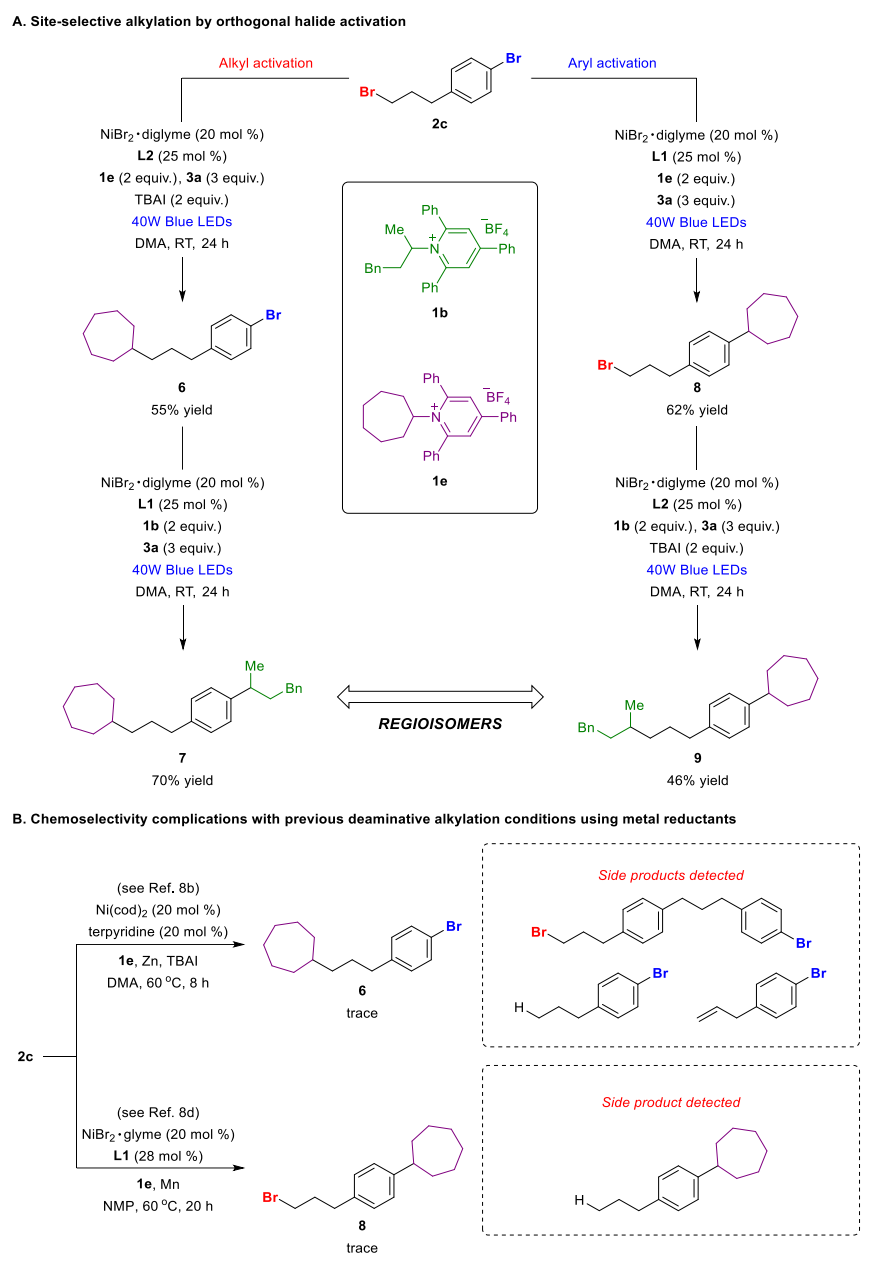

Scheme 7. Site- and chemoselective incorporation of aliphatic groups by photoinduced catalytic cross-coupling.

\section{Acknowledgements}

This research was supported by the National University of Singapore Academic Research Fund Tier 1: R-143-000-B57-114 (M.J.K.).

Keywords: Nickel • Photochemistry - Electron donor-acceptor complex $\bullet$ Cross-coupling $\cdot$ Pyridinium salt

[1] For reviews of transition metal-catalyzed cross-coupling reactions, see: a) Metal-Catalyzed Cross-Coupling Reactions, 2nd ed., ed. by A. de Meijere, F. Diederich, Wiley-VCH,Weinheim, 2004; b) Metal-Catalyzed Cross-Coupling Reactions, ed. by F. Diederich, P. J. Stang, Wiley-VCH, Weinheim, 1998; c) Handbook of Organopalladium Chemistry for Organic Synthesis, ed. by E. Negishi, A. de Meijere, Wiley-Interscience, New York, 2002, p. 1669; d) C. Liu, H. Zhang, W, Shi, A. Lei, Chem. Rev. 2011, 111, 1780; e) R. Jana, T. Pathak, M. S. Sigman. Chem. Rev. 2011, 111, 1417.

[2] For selected reviews see: a) A. Meijere, F. E. Meyer, Angew. Chem. Int. Ed. 1994, 33, 2379; b) K. C. Nicolaou, P. G. Bulger, D. Sarlah, Angew. Chem. Int. Ed. 2005, 44, 4442; c) A. B. Dounay, L. E. Overman, Chem. Rev. 2003, 103, 2945; d) K. W. Quasdorf, L. E. Overman, Nature 2014, $516,181$.

[3] For selected reviews and examples, see: a) D. J. Weix, Acc. Chem. Res. 2015, 48, 1767; b) J. Gu, X. Wang, W. Xue, H. Gong, Org. Chem. Front.
2015, 2, 1411; c) C. E. I. Knappke, S. Grupe, D. Gartner, M. Corpet, C. Gosmini, A. von. Jacobi von Wangelin, Chem.-Eur. J. 2014, 20, 6828; d) K. E. Poremba, S. E. Dibrell, S. E. Reisman, ACS Catal. 2020, 10, 8237; e) M. J. Goldfogel, L. Huang, D. J. Weix, In Nickel Catalysis in Synthesis: Methods and Reactions; Ogoshi, S., ed.; Wiley-VCH: Weinheim, 2020; 352.

[4] For cross-coupling of two organohalides, selected examples, see: a) D. A. Everson, R. Shrestha, D. J. Weix, J. Am. Chem. Soc. 2010, 132, 920; b) X. Yu, T. Yang, S. Wang, H. Xu, H. Gong, Org. Lett. 2011, 13, 2138; c) D. A. Everson, B. A. Jones, D. J. Weix, J. Am. Chem. Soc. 2012, 134 6146; d) S. Biswas, D. J. Weix, J. Am. Chem. Soc. 2013, 135, 16192; e) X. Wang, G. Ma, Y. Peng, C. E. Pitsch, B. J. Moll, T. D. Ly, X. Wang, H. Gong, J. Am. Chem. Soc. 2018, 140, 14490; f) J. Liu, Q. Ren, X. Zhang, H. Gong, Angew. Chem. Int. Ed. 2016, 55, 15544; g) A. H. Cherney, N. T. Kadunce, S. E. Reisman, J. Am. Chem. Soc. 2013, 135, 7442; h) S. Kim, M. J. Goldfogel, M. M. Gilbert, D. J. Weix, J. Am. Chem. Soc. 2020, 142, 9902; i) H. A. Sakai, W. Liu, C. "C." Le, D. W. C. MacMillan, J. Am. Chem. Soc. 2020, 142, 11691; j) J. Sheng, H.-Q. Ni, H.-R. Zhang, K.-F. Zhang, Y.-N. Wang, X.-S. Wang, Angew. Chem. Int. Ed. 2018, 57, 7634; k) F. Chen, K. Chen, Y. Zhang, Y. He, Y.-M. Wang, S. Zhu, J. Am. Chem. Soc. 2017, 139, 13929.

[5] For cross-coupling of organohalides and pseudohalides, selected examples, see: a) H. Chen, X. Jia, Y. Yu, Q. Qun, H. Gong, Angew. Chem. Int. Ed. 2017, 56, 13103; b) K. M. Arendt, A. G. Doyle, Angew. Chem. Int. Ed. 2015, 54, 9876; c) M. O. Konev, L. E. Hanna, E. R. Jarvo, Angew. Chem. Int. Ed. 2016, 55, 6730; d) Y. Ye, H. Chen, J. L. Sessler, H. Gong, J. Am. Chem. Soc. 2019, 141, 820; e) L. K. G. Ackerman, M. M. Lovell, D. J. Weix, Nature 2015, 524, 454; f) L. Huang, L. K. G. Ackerman, K. Kang, A. Parsons, D. J. Weix, J. Am. Chem. Soc. 2019, 141, 10978; g) K. Kang, L. Huang, D. J. Weix, J. Am. Chem. Soc. 2020, 142, 10634; h) J. H. Liu, C. T. Yang, X. Y. Lu, Z. Q. Zhang, L. Xu, M. Cui, X. Lu, B. Xiao, Y. Fu, L. Liu, Chem. Eur. J. 2014, 20, 15334; i) F. Cong, X.-Y. Lv, C. S. Day, R. Martin, J. Am. Chem. Soc. 2020, 142, 20594; j) V. R. Bhonde, B. T. OÏNeill, S. L. Buchwald, Angew. Chem. Int. Ed. 2016, $55,1849$.

[6] For cross-coupling of organohalide and redox-active esters, selected examples, see: a) K. M. M. Huihui, J. A. Caputo, Z. Melchor, A. M. Olivares, A. M. Spiewak, K. A. Johnson, T. A. DiBenedetto, S. Kim, L. K. G. Ackerman, D. J. Weix, J. Am. Chem. Soc. 2016, 138, 5016; b) L. Huang, A. M. Olivares, D. J. Weix, Angew. Chem. Int. Ed. 2017, 56, 11901 ; c) N. Suzuki, J. L. Hofstra, K. E. Poremba, S. E. Reisman, Org. Lett. 2017, 19, 2150.

[7] a) A. R. Katritzky, G. De Ville, R. C. Patel, Tetrahedron 1981, 37, 25. b) J. B. Bapat, R. J. Blade, A. J. Boulton, J. Epsztajn, A. R. Katritzky, J. Lewis, P. Molina-Buendia, P.-L. Nie, C. A. Ramsden, Tetrahedron Lett. 1976, 17, 2691; c) A. R. Katritzky, C. M. Marson, Angew. Chem. Int. Ed. 1984, 23, 420; d) C. H. Basch, J. Liao, J. Xu, J. J. Piane, M. P. Watson, J. Am. Chem. Soc. 2017, 139, 5313.

[8] a) H. Yue, C. Zhu, L. Shen, Q. Geng, K. J. Hock, T. Yuan, L. Cavallo, M. Reuping, Chem. Sci. 2019, 10, 4430; b) S. Ni, C. X. Li, Y. Mao, J. Han, Y. Wang, H. Yan, Y. Pan, Sci. Adv. 2019, 5: eaaw9516; c) J. Liao, C. H. Basch, M. E. Hoerrner, M. R. Boscoe, J. W. Tucker, M. R. Garnsey, M. P. Watson; Org. Lett. 2019, 21, 2941; d) R. Martin-Montero, V. Reddy Yatham, H. Yin, J. Davies, R. Martin, Org. Lett. 2019, 21, 2947; e) J. Yi, S. O. Badir, L. M. Kammer, M. Ribagorda, G. A. Molander, Org. Lett. 2019, 21, 3346.

[9] For previous reports employing TDAE with nickel catalysis, see: a) L. Anka-Lufford, K. M. M. Huihui, N. J. Gower, L. K. G. Ackerman, D. J Weix, Chem. - Eur. J. 2016, 22, 11564; b) J. Broggi, T. Terme, P. Vanelle, Angew. Chem., Int. Ed. 2014, 53, 384.

[10] H. Xu, C. Zhao, Q. Qian, W. Deng, H. Gong, Chem. Sci. 2013, 4, 4022.

[11] a) J. Yin, C. K. Maguire, N. Yasuda, A. P. J. Brunskill, A. Klapars, Org. Process Res. Dev. 2017, 21, 94; b) K. Takai, T. Kakiuchi, K. A. Utimoto, J. Org. Chem. 1994, 59, 2671; c) M. Durandetti, C. Gosmini, J. Perichon, Tetrahedron 2007, 63, 1146; d) M. Amatore, C. Gosmini, Chem. - Eur. J. 2010, 16, 5848; e) S. Wang, Q. Qian, H. Gong, Org. Lett. 2012, 14, 3352.

[12] M. Acemoglu, C. M. Krell, W. Marterer, Transition Metal-Catalyzed Couplings in Process Chemistry; Magano, J., Dunetz, J. R., Eds.; WileyVCH: Weinheim, Germany, 2013; pp 15-23.

[13] S.L. O'Neal, W. Zheng, Curr. Envir. Health Rpt. 2015, 2, 315-328. 
[14] a) S. L. Rössler, B. J. Jelier, E. Magnier, G. Dagousset, E. M. Carreira, A. Togni, Angew. Chem. Int. Ed. 2020, 59, 9264; b) S. Plunkett, C. H. Basch, S. O. Santana, M. P. Watson, J. Am. Chem. Soc. 2019, 141, 2257; c) S. Z. Sun, C. Romano, R. Martin, J. Am. Chem. Soc. 2019, 141, 16197; d) M. M. Zhang, F. Liu, Org. Chem. Front. 2018, 5, 3443; e) F. J. R. Klauck, H. Yoon, M. J. James, M. Lautens, F. Glorius, ACS Catal. 2019, 9, 236 f) K. M. Baker, D. L. Baca, S. P., M. E. Daneker, Mary P. Watson; Org. Lett. 2019, 21, 9738; g) M. A. Ashley, T. Rovis, J. Am. Chem. Soc. 2020, $142,18310$.

[15] a) J. Liao, W. Guan, B. P. Boscoe, J. W. Tucker, J. W. Tomlin, M. R. Garnsey, M. P. Watson, Org. Lett. 2018, 20, 3030; b) M. E. Hoerrner, K. M. Baker, C. H. Basch, E. M. Bampo, M. P. Watson, Org. Lett. 2019, 21 , 7356.

[16] a) F. J. R. Klauck, M. J. James, F. Glorius, Angew. Chem. Int. Ed. 2017, 56, 12336; b) X. Jiang, M.-M. Zhang, W. Xiong, L.-Q. Lu, W.-J. Xiao; Angew. Chem. Int. Ed. 2019, 58, 2402; c) Q. Xia, Y. Li, X. Wang, P. Dai, H. Deng, W.-H. Zhang, Org. Lett. 2020, 22, 7290.

[17] J. Wu, L. He, A. Noble, V. K. Aggarwal, J. Am. Chem. Soc. 2018, 140, 10700.

[18] J. Wu, P. S. Grant, X. Li, A. Noble, V. K. Aggarwal, Angew. Chem. Int. Ed. 2019, 58, 5697.

[19] a) S. M. Goldup, D. A. Leigh, R. T. McBurney, P. R. McGonigal, A. Plant, Chem. Sci. 2010, 1, 383; b) M. R. Prinsell, D. A. Everson, D. J. Weix, Chem. Commun. 2010, 46, 5743

[20] a) J. -R. Wang, K. Manabe, Synthesis 2009, 9, 1405; b) M. W. Giuliano, S. J. Miller; Top Curr. Chem. 2016, 372, 157. 



\section{Table of Contents}

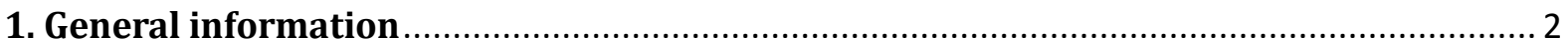

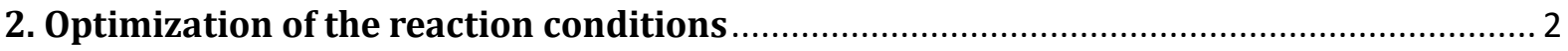

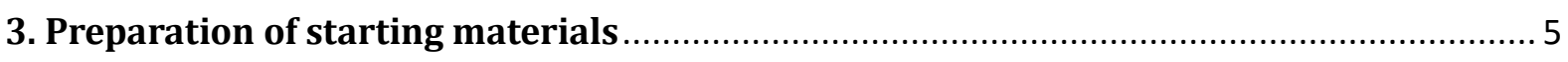

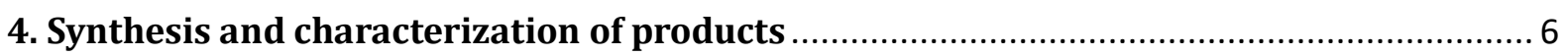

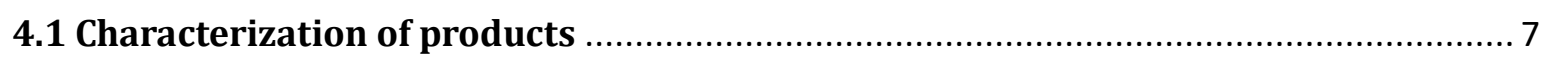

4.2 Site-selective alkylation by orthogonal halide activation ........................................ 23

4.3 Attempted deaminative alkylation using metal reductants...................................... 25

4.4 Attempted cross-coupling to access 4at using previous reductive methods............. 25

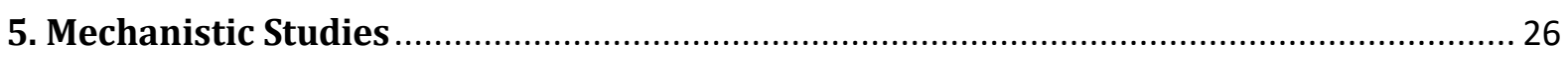

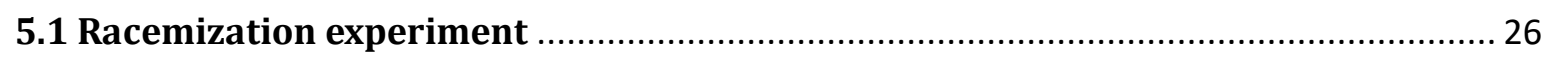

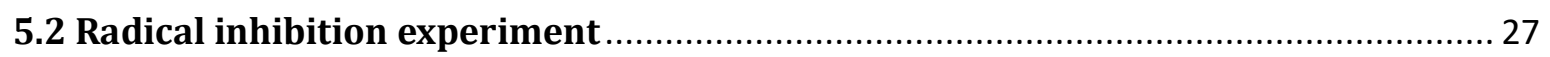

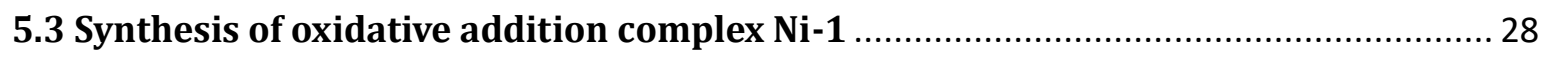

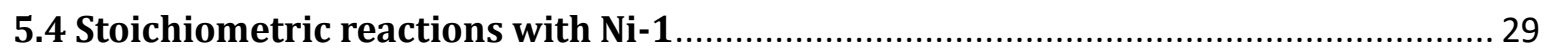

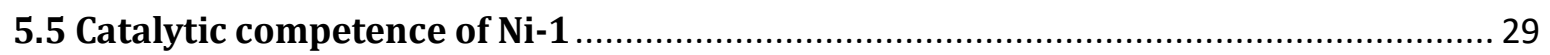

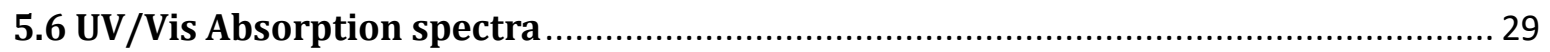

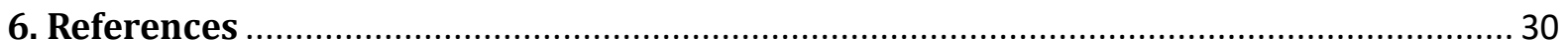

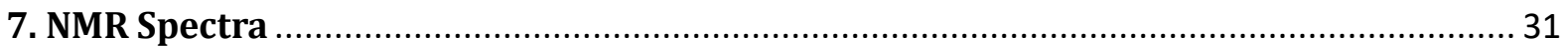




\section{General information}

All commercial reagents were used without additional purification, unless otherwise stated. Anhydrous solventwas purchased from commercial sources and transferred under an argon atmosphere. NMR spectra were recorded on Bruker $400 \mathrm{MHz}$ and Bruker DPX $500 \mathrm{MHz}$ spectrometer. Chemical shifts are reported in ppm from tetramethyl silane with the solvent resonance resulting from incomplete deuterium incorporation as the

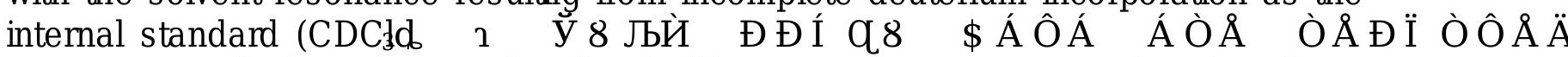
integration, multiplicity $(\mathrm{s}=$ singlet, $\mathrm{d}=$ doublet, $\mathrm{t}=$ triplet, $\mathrm{q}=$ quartet, $\mathrm{br}=$ broad, $\mathrm{m}=$ multiplet), and coupling constants (Hz). 13C NMR spectra were recorded on Bruker 400 $\mathrm{MHz}$ and Bruker DPX $500 \mathrm{MHz}$ spectrometer with complete proton decoupling. Chemical shifts are reported in ppm from tetramethyl silane with the solvent resonance as the internal standard (CDCldo $1.00 \breve{p p} \mathrm{p}$ obtained using Agilent 7200 QTOF and Bruker MicroTOFll. GEMS analysis was performed on Shimadzu GCM\$P2020 gas chromatography coupled to a Shimadzu QP2010 mass selective detector. Column conditionare reported in the experimental section below. Values foregioisomeric ratio of products were determined by GC (GC QP2030) and 1H NMR analysis.

Solvents

Solvents (acetonitrile, $\mathrm{CH}_{2} \mathrm{Cb}$, diethyl ether, tetrahydrofuran) were purified under a positive pressure of dry nitrogen gas by a modified Innovative Tohnologies purification system. N,N-Dimethylacetamide (anhydrous), N,N-dimethylformamide (anhydrous), dimethyl sulfoxide (anhydrous), dimethoxyethane (anhydrous), and 1,4lioxane (anhydrous) were used asreceived. All purification procedures of products were carried out with reagent grade solvents.

Materials

Unless otherwise noted, commercial reagents were purchased from Aldrich, Alfa Aesar, or other commercial suppliers and were used as receivedAnhydrous NiCb.DME and anhydrous $\mathrm{NiBr}_{2}$ (diglyme) were purchased from Sigma Aldrich.

\section{Optimization of the reaction conditions}

\section{General procedure for optimization of cross-coupling of pyridinium salts with aryl bromides}

To an ovendried $5 \mathrm{~mL}$ vial equipped with a magnetic stir bar was added aryl bromide $(0.1 \mathrm{mmol})$, pyridinium salts $(0.13 \mathrm{mmol}), \mathrm{HE}(0.2 \mathrm{mmol})$, and Ligand $(0.11 \mathrm{mmol})$. The vial was transferred to a $\mathrm{N}$-filled glovebox. $\mathrm{NiBr}$ (diglyme) $(0.01 \mathrm{mmol}$ ) was added to the vial, and the mixture was then dssolved in $1.0 \mathrm{mIsolvent}$. The vial was tightly sealed and removed from the glovebox. Te reaction was allowed to stir under $40 \mathrm{~W}$ blue LED irradiation for $16 \mathrm{~h}$ with fans to maintain the temperature below $30^{\circ} \mathrm{C}$ (the vial was placed about $3 \mathrm{~mm}$ from the LEDssee experimental setup)After that, the reaction was diluted with $6 \mathrm{~mL}$ EtOAc, and washed with saturatedq. $\mathrm{NaCl}(2 \times 3 \mathrm{~mL})$, the organic was separated and concentrated under reduced pressure he residue was subjected to flash silica gel columnchromatography to afford the pure product. 
Table S1 Optimization of cross-coupling of secondary pyridinium salts with aryl bromides $^{\mathrm{a}}$<smiles>COC(=O)c1ccc(Br)cc1</smiles><smiles>O=C(Oc1ccccc1)OC(F)(F)F</smiles>

$\mathrm{NiBr}_{2} \cdot$ diglyme $(10 \mathrm{~mol} \%)$ L1 (11 mol\%), 3a (2.0 eq.) DMA, 40 W Blue LED, rt. $16 \mathrm{~h}$<smiles>CC(=O)c1ccc(C2CCN(C(C)(C)C)CC2)cc1</smiles>
1.0 eq $1.3 \mathrm{eq}$

\begin{tabular}{|c|l|c|}
\hline Entry & Deviation standard conditions & Yield (\%) $^{\mathrm{b}}$ \\
\hline 1 & none & 85 \\
\hline 2 & L2 instead of L1 & 81 \\
\hline 3 & L3 instead of L1 & 68 \\
\hline 4 & NiCl $_{2}$ DME instead of $\mathrm{NiBr}_{2}$ 'diglyme & 77 \\
\hline 5 & DMF as solvent & 20 \\
\hline 6 & DMSO as solvent & 0 \\
\hline 7 & MeCN as solvent & trace \\
\hline 8 & THF as solvent & 0 \\
\hline 9 & TMEDA instead of 3a & 0 \\
\hline 10 & No Ni, L1 3a or light & 78 \\
\hline 11 & 0.5 mL DMA was used & . \\
\hline
\end{tabular}<smiles>[R]c1ccnc(-c2cc([R])ccn2)c1</smiles>

$\mathrm{L} 1 \mathrm{R}=\mathrm{OMe}$ $\mathrm{L} 3 \mathrm{R}={ }^{t} \mathrm{Bu}$ L4 $\mathrm{R}=\mathrm{H}$<smiles>CCOC(=O)C1=C(C)NC(C)=C(C(=O)OCC)C1</smiles>
a Reaction Conditions $\mathbf{2 a}(0.1 \mathrm{mmol})$, 1a $(0.13 \mathrm{mmol})$, Ni cat. (10 mol\%) Ligand (11 mol\%) 3a $(0.2 \mathrm{mmol})$, in solvent (1.0 mL) atRT under $40 \mathrm{~W}$ blue LED irradiation for $16 \mathrm{hb}$ Isolated yield.

Table S2 Optimization of cross-coupling ofprimary pyridinium salts with aryl bromides
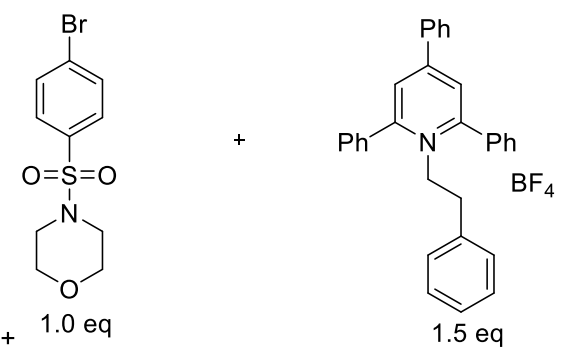

$\mathrm{NiBr}_{2} \cdot$ diglyme (10 mol\%) / L (12 mol\%)

3a (2.0 eq), DMA $(0.8 \mathrm{~mL})$, additive (3.0 eq),

$40 \mathrm{~W}$ blue LED, rt, $24 \mathrm{~h}$

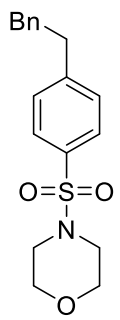

\begin{tabular}{|c|c|c|c|}
\hline entry & $\mathrm{L}$ & additive & Yield (\%) ${ }^{b}$ \\
\hline 1 & L1 & none & 0 \\
\hline 2 & L2 & none & 0 \\
\hline 3 & $\mathbf{L 3}$ & none & 0 \\
\hline 4 & $L 4$ & none & 0 \\
\hline 5 & $\mathbf{L 5}$ & none & 0 \\
\hline 6 & L6 & none & 0 \\
\hline 7 & L3 & TEA & $51^{c}$ \\
\hline 8 & L3 & DABCO & 6 \\
\hline 9 & L3 & ${ }^{\mathrm{i}} \mathrm{Pr}_{2} \mathrm{NEt}$ & 21 \\
\hline 10 & L3 & $\mathrm{K}_{2} \mathrm{CO}_{3}$ & 4 \\
\hline 11 & L3 & $\mathrm{K}_{3} \mathrm{PO}_{4}$ & 10 \\
\hline
\end{tabular}

a Reaction Conditions aryl bromide ( $0.1 \mathrm{mmol})$, py salt $(0.15 \mathrm{mmol})$, Ni cat. (10 mol\%), Ligand (12 $\mathrm{mol} \%)$, 3a $(0.2 \mathrm{mmol})$, and additive $(03 \mathrm{mmol})$ in DMA $(0.8 \mathrm{~mL})$ at RT under $40 \mathrm{~W}$ blue LED irradiation for $24 \mathrm{~h}$. Determined by GC analysis witm-tridecane as an intemal standardc Isolated yield. 


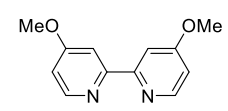

L1

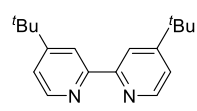

L3

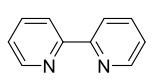

L4

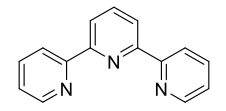

L5

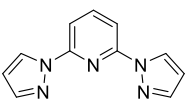

$\mathrm{L} 2$

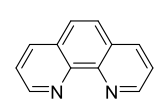

L6

\section{General procedure for optimization of cross-coupling of pyridinium salts with alkyl iodides}

To an ovendried $5 \mathrm{~mL}$ vial equipped with a magnetic stir bar was added alkyl iodide (0.1 $\mathrm{mmol})$, pyridinium salts $(0.15 \mathrm{mmol}), \mathrm{HE}(0.3 \mathrm{mmol})$, andigand $(0.025 \mathrm{mmol})$. The vial was transferred to a $\$$-filled glovebox. $\mathrm{NiBk}$ (diglyme) $(0.02 \mathrm{mmol})$ was added to the vial, and the mixture was then dissolved in 0.8 mIsolvent. The vial was tightly sealed and removed from the glovebox. Te reaction was allowed to stir under $40 \mathrm{~W}$ blue LED irradiation for $16 \mathrm{~h}$ with fans to maintain the temperature below $30{ }^{\circ} \mathrm{C}$ (the vial was placed about $3 \mathrm{~mm}$ from the LEDs, see experimental setupffter that, the reaction was diluted with $1 \mathrm{~mL}$ EtOAc, and them-tridecane (12 mg) as an internal standard was added

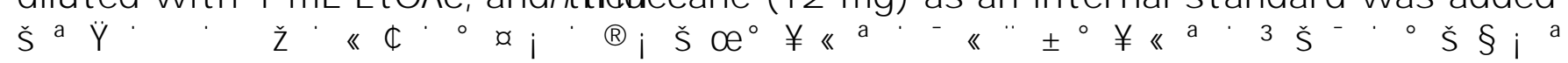

Table S3 Optimization of cross-coupling of pyridinium salts with alkyl iodides ${ }^{a}$

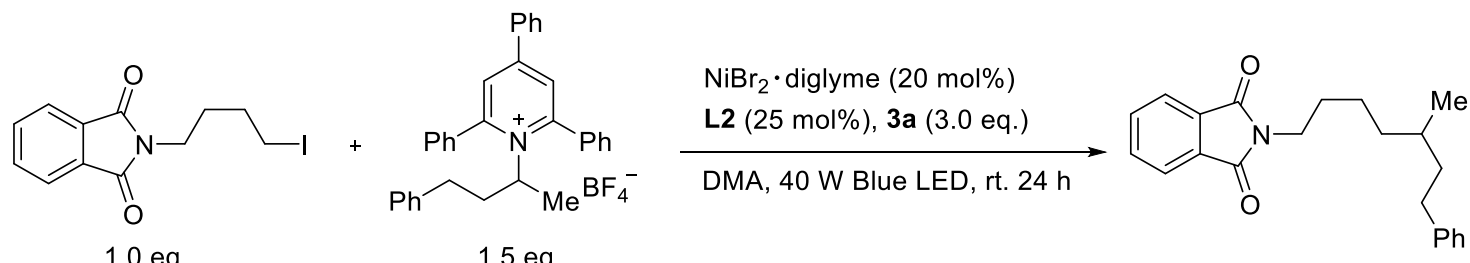

\begin{tabular}{|c|c|c|}
\hline Entry & Deviation standard conditions & Yield $(\%)^{b}$ \\
\hline 1 & none & $83 \%(76 \%)^{c}$ \\
\hline 2 & L5 instead of $\mathbf{L}$ & $5 \%$ \\
\hline 3 & $\mathbf{L 1}, \mathbf{L 3}$ or $\mathbf{L 4}$ instead of $\mathbf{L} \mathbf{2}$ & trace \\
\hline 5 & 10 mol\% $\mathrm{Ni}$ and $12.5 \mathrm{~mol} \% \mathbf{L}$ & $62 \%$ \\
\hline 6 & $15 \mathrm{~mol} \% \mathrm{Ni}$ and $18.5 \mathrm{~mol} \% \mathbf{L}$ & $71 \%$ \\
\hline 7 & DMF as solvent & $6 \%$ \\
\hline 8 & NMP as solvent & trace \\
\hline 9 & Alkyl bromide was used & ND \\
\hline 10 & Alkyl bromide and Nal were used & trace \\
\hline 11 & $0.6 \mathrm{~mL}$ DMA was used & $77 \%$ \\
\hline 12 & 1.0 eq py salt was used & $55 \%$ \\
\hline 13 & 1.8 eq py salt was used & $85 \%$ \\
\hline $\mathrm{MeO}$ & $=\mathrm{N}_{\mathrm{L}}$ & \\
\hline
\end{tabular}

a Reaction Conditions alkyl iodide $(0.1 \mathrm{mmol})$, py salt $(0.15 \mathrm{mmol})$, Ni cat. $(20 \mathrm{~mol} \%)$, Ligand $(25 \mathrm{~mol} \%)$, 3a $(0.3 \mathrm{mmol})$, in DMA $(0.8 \mathrm{~mL})$ at RT under $40 \mathrm{~W}$ blue LED imadiation for 24 hDetermined by GC analysis with $\mathrm{n}$-tridecane as an internal standardc Isolated yield.

Table S4 Optimization of Electrondonors with aryl bromide ${ }^{a}$ 
<smiles>O=C(c1ccc(Br)cc1)N1CCOCC1</smiles><smiles>CC(CBr)[n+]1c(-c2ccccc2)cc(-c2ccccc2)cc1-c1ccccc1</smiles>
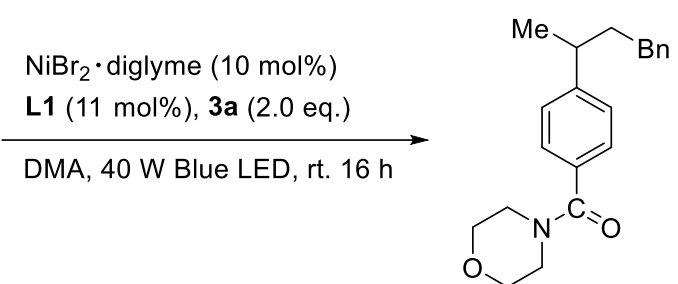

$1.0 \mathrm{eq}$

1.3 eq

\begin{tabular}{|c|l|c|}
\hline Entry & Deviation standard conditions & Yield (\%) \\
\hline 1 & none & 86 \\
\hline 2 & mNADH instead of 3a & 51 \\
\hline 3 & Et $3 \mathrm{~N}$ instead of 3a \\
\hline
\end{tabular}

a Reaction Conditions:aryl bromide $(0.1 \mathrm{mmol})$, py salt $(0.13 \mathrm{mmol}), \mathrm{NBr} \cdot \operatorname{diglyme}(10 \mathrm{~mol} \%), \mathbf{L 1}(11$ $\mathrm{mol} \%), 3 a(0.2 \mathrm{mmol})$, in DMA $(1.0 \mathrm{~mL})$ at RT under $40 \mathrm{~W}$ blue LED imadiation for 16 p.Isolated yield.

Table S5 Optimization of Electron donors with alkyl iodide

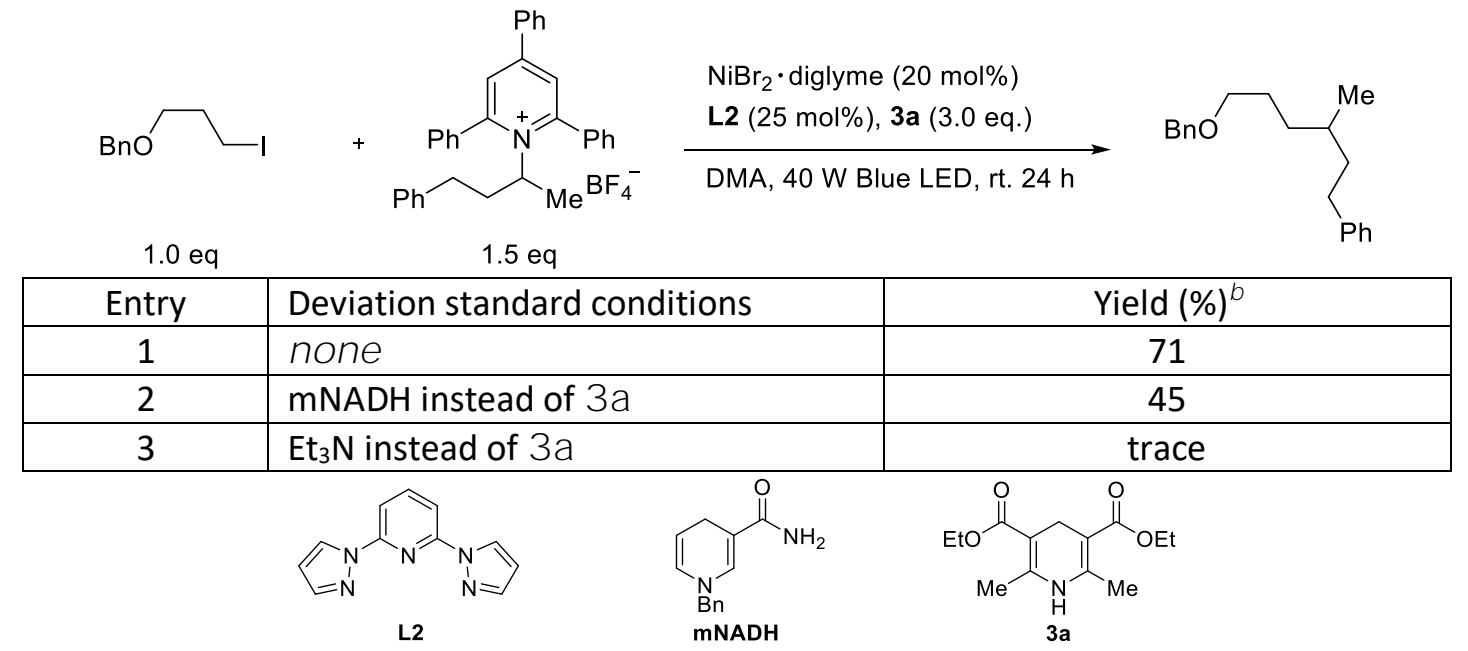

a Reaction Conditions:alkyl iodide $(0.1 \mathrm{mmol})$, py salt $(0.15 \mathrm{mmol})$, NiBr.diglyme $(20 \mathrm{~mol} \%)$, $\mathbf{L} 2$ (25 $\mathrm{mol} \%), 3 \mathbf{a}(0.3 \mathrm{mmol})$, in DMA $(0.8 \mathrm{~mL})$ at RT under $40 \mathrm{~W}$ blue LED irradiation for 24 hisolated yield.

\section{Preparation of starting materials}

\section{General Procedure 1 (GP1): Conversion of amines to pyridinium salts}

According to the procedure described by Watson et alPrimary amine (1.2 eq.) was added to a suspension of 2,4-6riphenylpyrylium tetrafluoroborate (1.0 eq.) and EtOH $(1.0 \mathrm{M})$ in a long Schlentube. The mixture was stirred and heated at reflux in an oil bath at $85^{\circ} \mathrm{C}$ to $90^{\circ} \mathrm{C}$ for $4 \mathrm{~h}$. The mixture was then lbowed to cool to room temperature. If product precipitation occurred after cooling to room temperature, the solid was filtered, washed with EtOH $(3 \times 5 \mathrm{~mL})$ and then $\mathrm{E} \Theta(3 \times 25 \mathrm{~mL})$, and dried under high vacuum. If product precipitation did not occur during reflux, the solution was diluted with $\mathrm{E} \nsubseteq \mathrm{O}$ $\left(2 z^{3} \times\right.$ volume of EtOH used) and vigorously stirred for $1 \mathrm{~h}$ to induce trituration. The resulting solid pyridinium salt was filtered and washed with $\mathrm{E} \nsubseteq \mathrm{O}(3 \times 25 \mathrm{~mL})$. If the pyridinium salt failed to precipitate at this point, it was subjected to flash column chromatography, eluting with acetone/DCM 
Pyridinium salts used in thisstudy1,2,3,4.<smiles>CC(=O)OC(=O)N1CCC([n+]2c(-c3ccccc3)cc(-c3ccccc3)cc2-c2ccccc2)CC1</smiles>

Py 51<smiles>CC(C1CCCCC1)[n+]1c(-c2ccccc2)cc(-c2ccccc2)cc1-c1ccccc1</smiles>

Py S6<smiles>FC(F)(F)c1cc(-c2ccccc2)cc(-c2ccccc2)[n+]1C1CCCCC1</smiles>

Py S2<smiles>FC(F)(F)c1cc(-c2ccccc2)cc(-c2ccccc2)[n+]1C1CCCC1</smiles>

Py 57<smiles>CC(CCc1ccccc1)[n+]1c(-c2ccccc2)cc(-c2ccccc2)cc1-c1ccccc1</smiles>

Py S3<smiles>CCC(CO)[n+]1c(-c2ccccc2)cc(-c2ccccc2)cc1-c1ccccc1</smiles>

Py S8<smiles>O=C1CCC([n+]2c(C(=O)c3ccccc3)cc(-c3ccccc3)cc2-c2ccccc2)O1</smiles>

Py 54<smiles></smiles>

Py S5<smiles>FC(F)(F)c1cc(-c2ccccc2)cc(-c2ccccc2)[n+]1C1CCCCC1</smiles>

Py 59<smiles>CCCCCC(C)[n+]1c(-c2ccccc2)cc(-c2ccccc2)cc1-c1ccccc1</smiles>

PyS10<smiles>Cc1cccc(C)c1OCC(C)[n+]1c(-c2ccccc2)cc(-c2ccccc2)cc1-c1ccccc1</smiles>

Py S11<smiles>FC(F)(F)c1cc(-c2ccccc2)cc(-c2ccccc2)[n+]1CCc1ccccc1</smiles>

Py S14<smiles>CCC(COC(=O)Cc1ccc2c(c1)C(=O)c1ccccc1CO2)[n+]1c(-c2ccccc2)cc(-c2ccccc2)cc1-c1ccccc1</smiles>

Py S12<smiles>CC(C)(C)OC(=O)C[C@H]1C[C@H](CC[n+]2c(-c3ccccc3)cc(-c3ccccc3)cc2-c2ccccc2)OC(C)(C)O1</smiles>

Py $\mathrm{S} 15$<smiles></smiles>

Py S16<smiles>CCC(COC(=O)Cc1c(C)n(C(=O)c2ccc(Cl)cc2)c2ccc(OC)cc12)[n+]1c(-c2ccccc2)cc(-c2ccccc2)cc1-c1ccccc1</smiles>

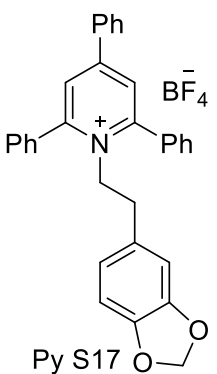

\section{Synthesis and characterization of products}

General Procedure 2 (GP2): Cross-coupling of pyridinium salts with aryl bromides To an ovendried $5 \mathrm{~mL}$ vial equipped with a magnetic stir bar was added aryl bromide (0.1 mmol), pyridinium salts $(0.13 \mathrm{mmol})$, HE $(0.2 \mathrm{mmol})$, and4,4'-dimethoxy-2,2'bipyridine $(0.011 \mathrm{mmol})$. The vial wastransferred to a $\mathrm{k}$-filled glovebox. $\mathrm{NiBk}$ (diglyme) (0.01 mmol) was added to the vial, andhte mixture was then dissolved in1.0 mL DMA. The vial was tightly sealed and removed from the gloveborthe reaction was allowed to stir under $40 \mathrm{~W}$ blue LED irradidion for $16 \mathrm{~h}$ with fans to maintain the temperature below $30{ }^{\circ} \mathrm{C}$ (the vial was placed about $3 \mathrm{~mm}$ from the LEDs, see experimental setupfter that, the reaction was diluted with $6 \mathrm{~mL}$ EtOAc, and washed withaturated aq. $\mathrm{NaCl}(2 \times 3 \mathrm{~mL})$, the organic was separated and concentrated under reduced pressurthe residue was subjected to flash silica gel column chromatography to afford the pure product. 
General Procedure 3 (GP3): Cross-coupling of pyridinium salts with alkyl iodides To an oven-dried $5 \mathrm{~mL}$ vial equipped with a magnetic stir bar was added alkyl iodide $(0.1$ mmol), pyridinium salts $(0.15 \mathrm{mmol}), \mathrm{HE}(0.3 \mathrm{mmol})$, and 2 , $6 \mathrm{li}(1 \mathrm{H}-$ pyrazol-1yl)pyridine $(0.025 \mathrm{mmol})$. The vial was transferred to a $\mathrm{N}$-filled glovebox. $\mathrm{NiBr}$ (diglyme) $(0.02 \mathrm{mmol})$ was added to the vial, andhte mixture was then dissolved in $0.8 \mathrm{~mL}$ DMA. The vial was tightly sealed and removed from the glovebox.he reaction was allowed to stir under $40 \mathrm{~W}$ blue LED irradiation for $4 \mathrm{~h}$ with fans to maintain the temperaturebelow $30{ }^{\circ} \mathrm{C}$ (the vial was placed about $3 \mathrm{~mm}$ from the LEDs, see experimental setuffter that, the reaction was diluted with $6 \mathrm{~mL}$ EtOAc, and washed with saturaterd. $\mathrm{NaCl}(2 \times 3 \mathrm{~mL})$, the organic was separated and concentrated under reduced pressurEhe residue was subjected to flash silica gel column chromatography to afford the pure product.

\section{Photochemical Reaction Setup}
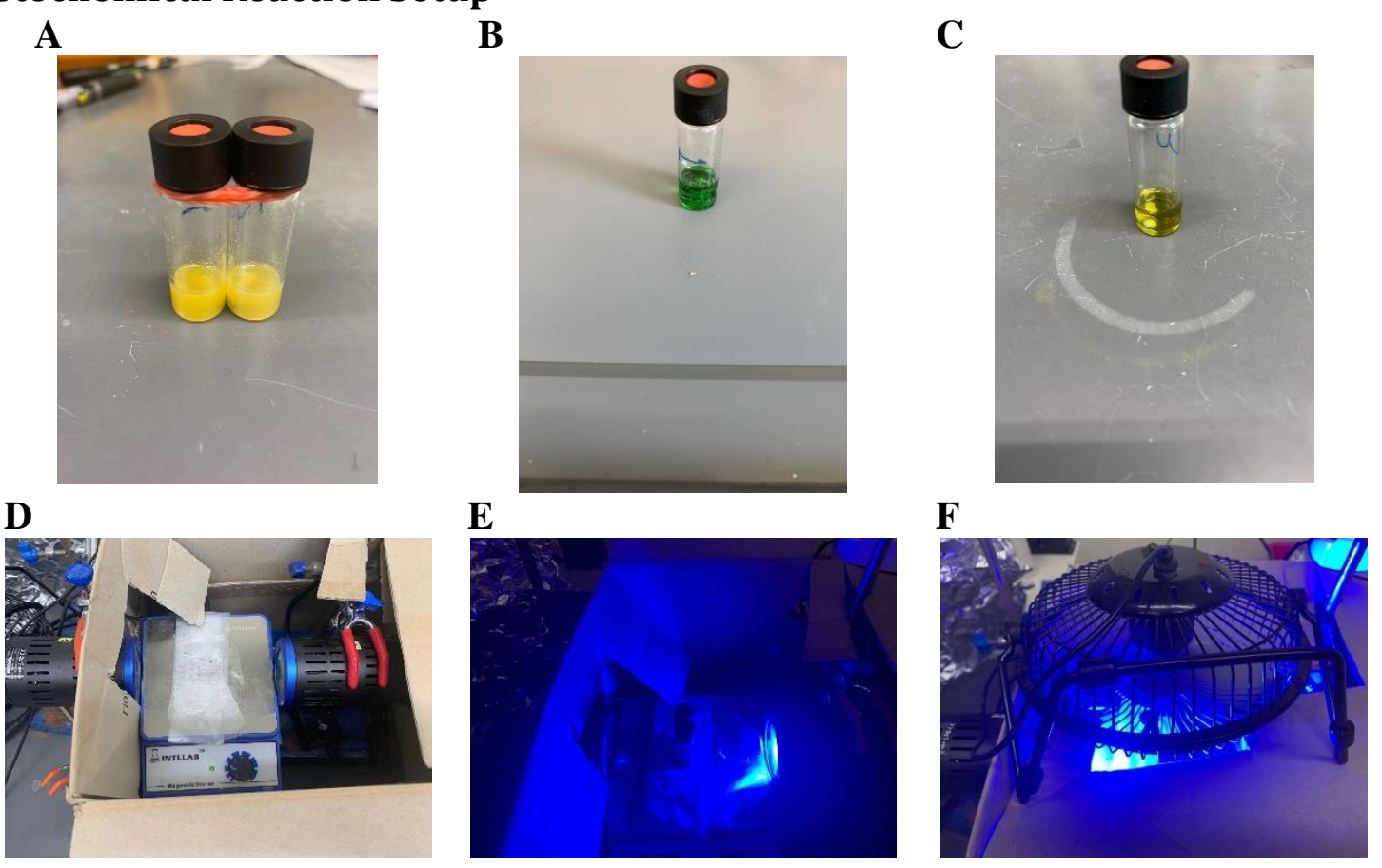

A. before reaction.B. Pyridinium salt with aryl bromide, after irradiation for 16 h. $\mathbf{C}$ Pyridinium salt with alkyl iodide, after irradiation for $24 \mathrm{~h}$. DEF. experiment setup.

\subsection{Characterization of products}

tent-Butyl 4-(4-(methoxycarbonyl)phenyl)piperidine-1-carboxylate $(4 a)^{5}$ :

Following the GP2, the title compound was prepared from methyl 4bromobenzoate (21.5 $\mathrm{mg}$ ) and Py S1 (75.1 mg) Purification using flash silica gel column chromatography (eluent: 15:1 hexane:EtOAc) gave the pure product as a colorless $2 \mathrm{i} \nabla(1 \mathrm{mg}, 85 \%$ yield).

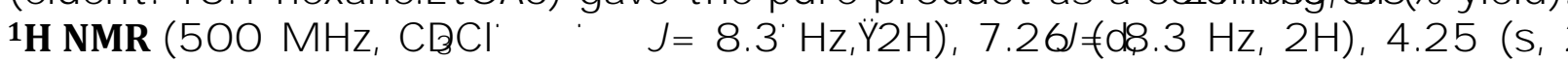
$3.89(\mathrm{~s}, 3 \mathrm{H}), 2.80(\mathrm{~s}, 2 \mathrm{H}), 2.70(\mathrm{t},=12.1,3.4 \mathrm{~Hz}, 1 \mathrm{H}), 1.82(\mathrm{~d},=12.8 \mathrm{~Hz}, 2 \mathrm{H}), 1.62(\mathrm{qd}$,

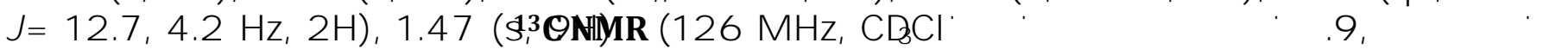
$128.3,126.8,79.5,52.0,44.2,42.8,32.8,28.4$ ppm. 
tert -Butyl 4-(4-cyanophenyl)piperidine-1-carboxylate $(4 \mathrm{c})^{5}$ :

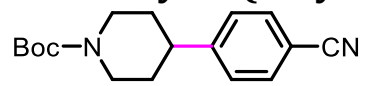

Following the GP2, the title compound was prepared from4-bromobenzonitrile (18.1 mg) and Py S1 (75.1 mg) Purification using flash silica gel column chromatography (eluent: 15:1 hexane:EtOAc) gave the pure product as a colorless di 8 (8 $\mathrm{mg}, 69 \%$ yield).

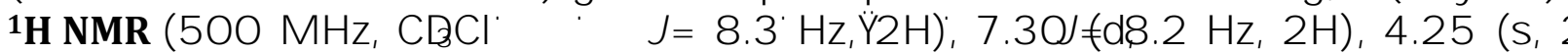
$2.79(\mathrm{~s}, 2 \mathrm{H}), 2.70(\mathrm{ttJ}=12.2,3.5 \mathrm{~Hz}, 1 \mathrm{H}), 1.80(\mathrm{~g},=13.0 \mathrm{~Hz}, 2 \mathrm{H}), 1.6 \notin 1.55(\mathrm{~m}, 2 \mathrm{H})$,

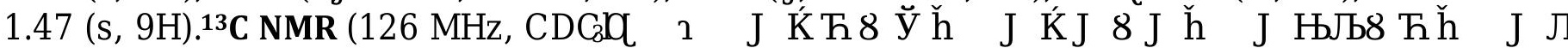
43.9, 42.9, 32.7, $28.4 \mathrm{ppm}$.

tert -Butyl 4-(4-formylphenyl)piperidine-1-carboxylate (4d) ${ }^{5}$ :

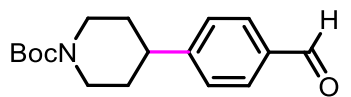

Following the GP2, the title compound was prepared from4-bromobenzaldehyde(18.4 mg) and Py S1 (75.1 mg) Purification using flash silica gel column chromatography (eluent: 15:1 hexane:EtOAc) gave the pure product as a colorless diR(2 mg, 42\% yield). ${ }^{1}$ H NMR (500 MHz, CDGQ 1 A 8 A y j $4.26(\mathrm{~s}, 2 \mathrm{H}), 2.81(\mathrm{~s}, 2 \mathrm{H}), 2.74(\mathrm{t},=12.1,3.5 \mathrm{~Hz}, 1 \mathrm{H}), 1.83(\mathrm{gl}=12.8 \mathrm{~Hz}, 2 \mathrm{H}), 1.6 \& 1.61$

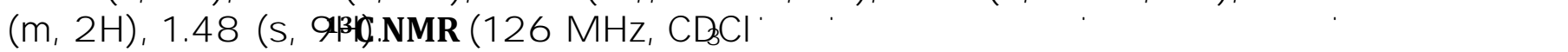
79.6, 44.1, 43.0, 32.8, $28.5 \mathrm{ppm}$.

tert -Butyl 4-(4-acetylphenyl)piperidine-1-carboxylate $(4 e)^{5}$ :

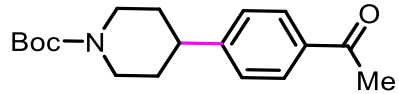

Following the GP2, the title compound was prepared from1-(4-bromophenyl)ethan-1one (20.0 mg) and Py S1 (75.1 mg) Purification using flash silica gel column chromatography (eluent: 15:1 hexane:EtOAc) gave the pure product as a colorless oil (21.2 mg, 70\%yield).

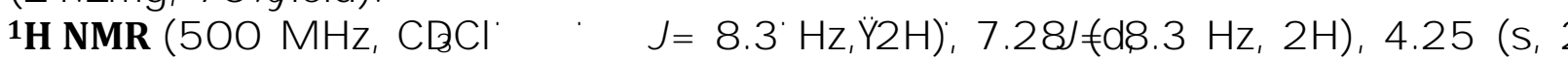
$2.80(\mathrm{~s}, 2 \mathrm{H}), 2.70(\mathrm{tt} J=12.0,3.5 \mathrm{~Hz}, 1 \mathrm{H}), 2.57(\mathrm{~s}, 3 \mathrm{H}), 1.831 .79(\mathrm{~m}, 2 \mathrm{H}), 1.67 \mathrm{z} 1.58(\mathrm{~m}$,

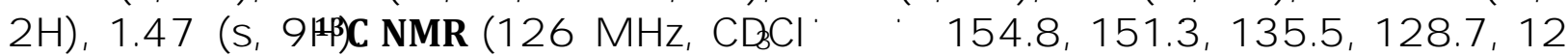
$79.5,44.2,42.8,32.8,28.4,26.5 \mathrm{ppm}$.

tert -Butyl 4-(4-(methylsulfonyl)phenyl)piperidine-1-carboxylate (4f) ${ }^{5}$ :<smiles>CC(C)(C)ON1CCC(c2ccc(S(=O)(=O)O)cc2)CC1</smiles>

Following the GP2, the title compound was prepared from1-bromo-4-(methylsulfonyl) benzene (23.5 mg) and Py S1 (75.1 mg) Purification using flash silica gel column chromatography (eluent: 5:1 hexane:EtOAc) gave the pure product as a colorless $2 \mathrm{~B} \mathbf{B} .(8$ mg, 76\% yield).

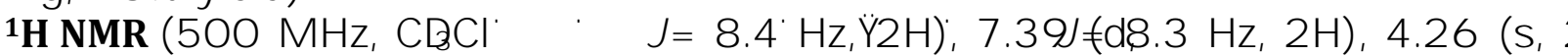
$3.03(\mathrm{~s}, 3 \mathrm{H}), 2.80(\mathrm{~s}, 2 \mathrm{H}), 2.782 .71(\mathrm{~m}, 1 \mathrm{H}), 1.82(\mathrm{dJ}=13.0 \mathrm{~Hz}, 2 \mathrm{H}), 1.6 \mathrm{Q} 1.58(\mathrm{~m}, 2 \mathrm{H})$,

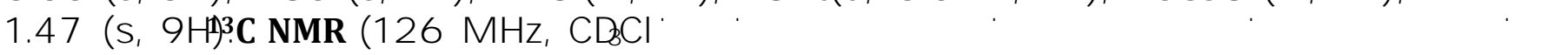
$44.0,42.7,328,28.4 \mathrm{ppm}$.

tert -Butyl 4-(4-(morpholinosulfonyl)phenyl)piperidine-1-carboxylate $(4 \mathrm{~g})^{6}$ :

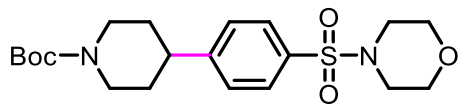


Following the GP2, the title compound was prepared from 4-((4-bromophenyl) sulfonyl)morpholine (31 $\mathrm{mg}$ ) and Py S1 (75.1 mg) Purification using flash silica gel column chromatography (eluent: 5:1 hexane:EtOAc) gave the pure product as a colorless oil ( $36.9 \mathrm{mg}, 90 \%$ yield).

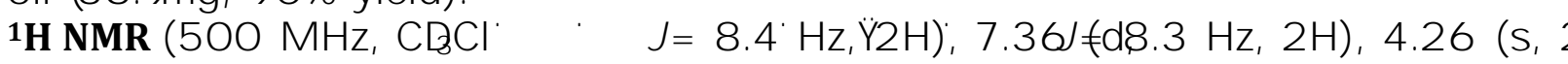
$3.74 \mathrm{z} 3.72(\mathrm{~m}, 4 \mathrm{H}), 2.99 \mathrm{z} 2.97(\mathrm{~m}, 4 \mathrm{H}), 2.80(\mathrm{~s}, 2 \mathrm{H}), 2.73(\mathrm{tg},=12.2,3.5 \mathrm{~Hz}, 1 \mathrm{H}), 1.82(\mathrm{~d}$, $\mathrm{J}=12.8 \mathrm{~Hz}, 2 \mathrm{H}), 1.62(\mathrm{qd},=12.7,4.2 \mathrm{~Hz}, 2 \mathrm{H}), 1.47(\mathrm{~s}, 9 \mathrm{H}) . \mathbf{C}$ NMR $(126 \mathrm{MHz}, \mathrm{CDG} \mathrm{Q}$ $154.7,151.3,133.0,128.2,127.5,976,66.0,45.9,44.1,42.7,32.8,28.4 \mathrm{ppm}$.

tert -Butyl 4-(4-(dibenzylcarbamoyl)phenyl)piperidine-1-carboxylate (4h)7:

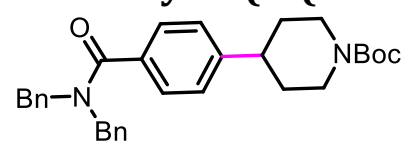

Following the GP2, the title compound was prepared from N,N-dibenzyl-4bromobenzamide( $38.0 \mathrm{mg}$ ) andPy S1 (75.1 mg)Purification using flash silica gel column chromatography (eluent: 15:1 hexane:EtOAc) gave the pure product as a colorless oil (32.0 mg, 66\% yield).

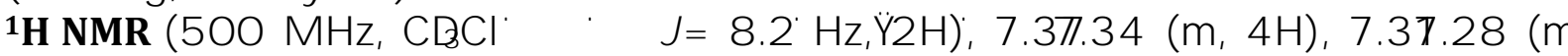
$4 \mathrm{H}), 7.20(\mathrm{~d} J=8.2 \mathrm{~Hz}, 2 \mathrm{H}), 7.15(\mathrm{dJ},=5.4 \mathrm{~Hz}, 2 \mathrm{H}), 4.69(\mathrm{~s}, 2 \mathrm{H}), 4.42(\mathrm{~s}, 2 \mathrm{H}), 4.23(\mathrm{~s}, 2 \mathrm{H})$, $2.78(\mathrm{~s}, 2 \mathrm{H}), 2.64(\mathrm{ttJ}=12.1,3.4 \mathrm{~Hz}, 1 \mathrm{H}), 1.79(\mathrm{~d},=12.1 \mathrm{~Hz}, 2 \mathrm{H}), 1.631 .60(\mathrm{~m}, 2 \mathrm{H})$,

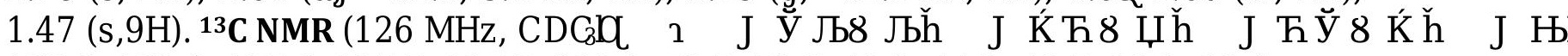
128.6, 128.4, 127.6, 127.5, 127.0, 126.9, 79.5, 51.6, 46.8, 44.3, 42.5, 32.9, 28.4 ppm.

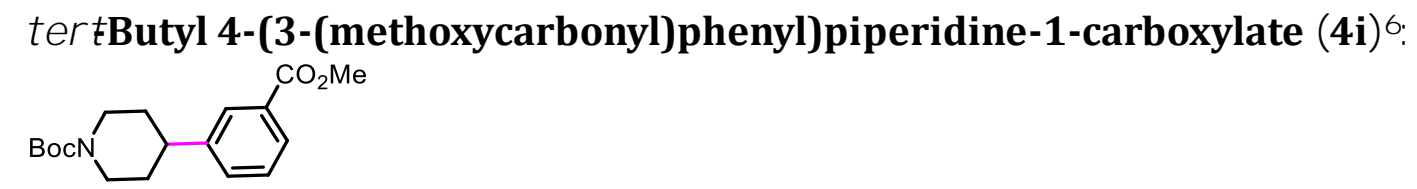

Following the GP2, the title compound was prepared frommethyl 3-bromobenzoate (21.5 mg) andPy S1 (75.1 mg)Purification using flash silica gel column chromatography (eluent: 15:1 hexane:EtOAc) gave the pure product ascalorless oil (25.2 $\mathrm{mg}, 79 \%$ yield).

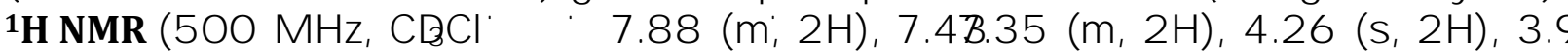
$3 \mathrm{H}), 2.80(\mathrm{~s}, 2 \mathrm{H}), 2.70(\mathrm{ttJ}=12.1,3.5 \mathrm{~Hz}, 1 \mathrm{H}), 1.83(\mathrm{~g},=12.9 \mathrm{~Hz}, 2 \mathrm{H}), 1.621 .63(\mathrm{~m}$,

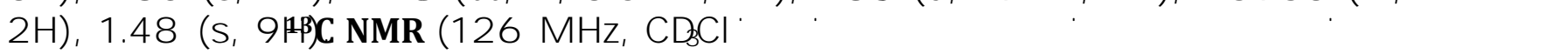
$128.0,127.6,79.5,52.1,44.3,42.5,33.0,28.5 \mathrm{ppm}$.

\section{tert -Butyl 4-(1-oxo-2,3-dihydro-1H-inden-5-yl)piperidine-1-carboxylate (4j):}

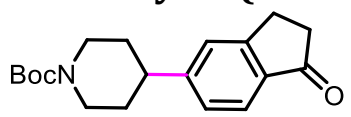

Following the GP2, the title compound was prepared from5-bromo-2,3-dihydro-1Hinden-1-one (21.1 mg) and Py S1 (75.1 mg),Purification using flash silica gel column chromatography (eluent: 15:1 hexane:EtOAc) gave the pure product as a colorless oil (26.2 $\mathrm{mg}, 83 \%$ yield).

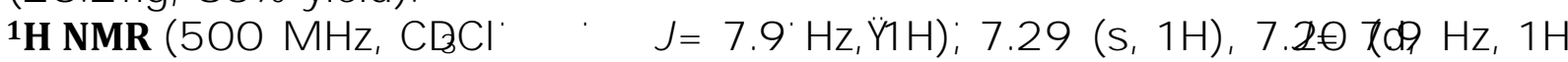
$4.25(\mathrm{~s}, 2 \mathrm{H}), 3.1 \mathrm{z} 3.09(\mathrm{~m}, 2 \mathrm{H}), 2.80(\mathrm{~s}, 2 \mathrm{H}), 2.72(\mathrm{tg},=12.1,3.4 \mathrm{~Hz}, 1 \mathrm{H}), 2.682 .65(\mathrm{~m}$, $2 \mathrm{H}), 1.82(\mathrm{~d} J=12.9 \mathrm{~Hz}, 2 \mathrm{H}), 1.68 \mathrm{c} 1.60(\mathrm{~m}, 2 \mathrm{H}), 1.47(\mathrm{~s}, 9 \mathrm{H}){ }^{13} \mathrm{C}$ NMR $\left(126 \mathrm{MHz}, \mathrm{CDG}_{\mathrm{B}}\right)$

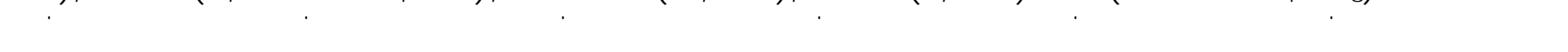
$25.7 \mathrm{ppm}$.

HRMS (ESI): Calcd. 338.1727 for $\mathrm{CH}_{25} \mathrm{NNaO}_{3}+(\mathrm{M}+\mathrm{Na})^{+}$, found 338.1729 . 
tert -Butyl 4-(6-(methoxycarbonyl)naphthalen-2-yl)piperidine-1-carboxylate (4k):<smiles>CC(=O)C1=Cc2ccc(C3CCN(C(=O)c4ccccc4)CC3)cc2CC1</smiles>

Following the GP2, the title compound was prepared frommethyl 6-bromo-2-naphthoate (26.5 mg) andPy S1 (75.1 mg)Purification using flash silica gelolumn chromatography (eluent: 15:1 hexane:EtOAc) gave the pure product as a colorless $2 i 8(1 \mathrm{mg}, 76 \%$ yield).

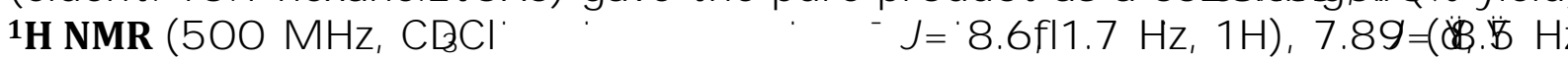
$1 \mathrm{H}), 7.82(\mathrm{~d} J=8.6 \mathrm{~Hz}, 1 \mathrm{H}), 7.66(\mathrm{~s}, 1 \mathrm{H}), 7.4(\mathrm{dd}, \mathrm{J}=8.5,1.7 \mathrm{~Hz}, 1 \mathrm{H}), 4.29(\mathrm{~s}, 2 \mathrm{H}), 3.97(\mathrm{~s}$, 3H), $2.86 \mathrm{z} 2.80(\mathrm{~m}, 3 \mathrm{H}), 1.91(\mathrm{dJ}=12.8 \mathrm{~Hz}, 2 \mathrm{H}), 1.73$ (ddd, =24.9, 12.4, $3.8 \mathrm{~Hz}, 2 \mathrm{H})$,

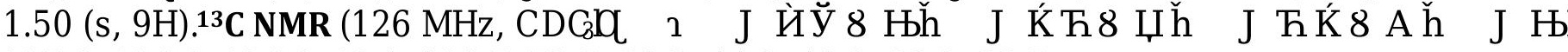
$127.8,126.9,126.6,12.4,124.7,79.5,52.2,44.3,42.9,33.0,28.5$ ppm.

HRMS (ESI): Calcd. 392.1832 for $62 \mathrm{H}_{27} \mathrm{NNaO}_{4}{ }^{+}(\mathrm{M}+\mathrm{Na})^{+}$, found 392.1829 .

tert-butyl 4-(4-((2-((4R,6R)-6-(2-(tert-butoxy)-2-oxoethyl)-2,2-dimethyl-1,3dioxan-4-yl)ethyl)carbamoyl)phenyl)piperidine-1-carboxylate (4l):

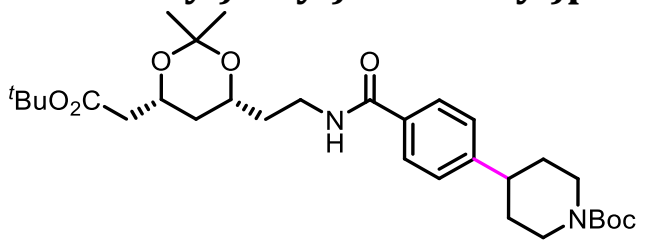

Following the GP2, the title compound was prepared from tert-butyl 2-(6-(2-(4bromobenzamido)ethyl)-2,2-dimethyl-1,3-dioxan-4-yl)acetate (46 mg) and Py S1 (75.1 mg), Purification using flash silica gel column chromatography (eluent: 5:1 hexane:EtOAc) gave the pure product as a colorless oal8(.4 mg, 52\% yield).

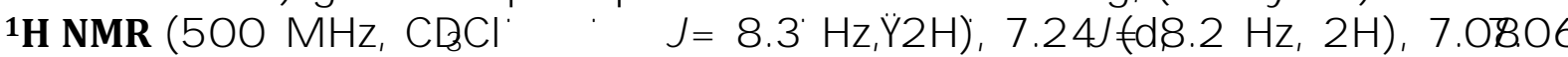
$(\mathrm{m}, 1 \mathrm{H}), 4.31 \mathrm{z} 4.25(\mathrm{~m}, 3 \mathrm{H}), 4.1 \mathrm{z} 4.07(\mathrm{~m}, 1 \mathrm{H}), 3.72(\mathrm{dddJ}=12.6,11.0,6.2 \mathrm{~Hz}, 1 \mathrm{H}), 3.41$ (qd, J = 8.4, 4.2 Hz, 1H), $2.79(\mathrm{~s}, 2 \mathrm{H}), 2.68(\mathrm{tt}=12.1,3.4 \mathrm{~Hz}, 1 \mathrm{H}) 2.43(\mathrm{dd}, \mathrm{J}=15.2,7.1$ $\mathrm{Hz}, 1 \mathrm{H}), 2.30(\mathrm{ddJ}=15.2,6.0 \mathrm{~Hz}, 1 \mathrm{H}), 1.8 \mathrm{Q} 1.79(\mathrm{~m}, 2 \mathrm{H}), 1.75 \mathrm{z} 1.54(\mathrm{~m}, 4 \mathrm{H}), 1.47(\mathrm{~s}$,

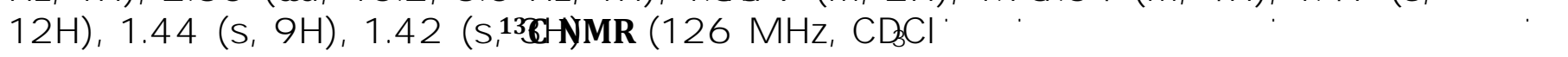
132.8, 127.0, 126.9, 98.8, 80.7, 79.5, 69.7, 6,644.1, 42.6, 42.5, 37.9, 36.0, 34.7, 32.9, 30.2, 29.7, 28.4, 28.1, $19.8 \mathrm{ppm}$.

HRMS (ESI): Calcd583.3354 for $\mathrm{C}_{31} \mathrm{H}_{48} \mathrm{~N}_{2} \mathrm{NaO}_{7}{ }^{+}(\mathrm{M}+\mathrm{Na})^{+}$, found 583.3348.

tert -Butyl $\quad 4-(4-c(((8 R, 9 S, 13 S, 14 \$-13-m e t h y l-17-0 x o-7,8,9,11,12,13,14,15,16,17-$ decahy dro-6H-cyclopenta[a]phenanthren-3-yl)oxy)carbonyl)phenyl)piperidine1-carboxylate $(4 \mathrm{~m})$ :

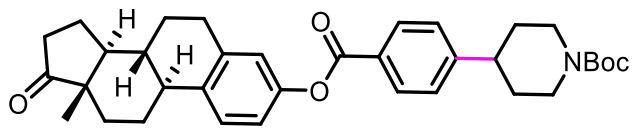

Following the GP2, the title compound was prepared from(8R,9S ,13S,14Sl 3-methyl-17oxo-7,8,9,11,12,13,14,15,16,1-7decahydro-6H-cyclopenta[a]phenanthren-3-yl 4-bromo benzoate (45.5 mg) and Py S1 (75.1 mg,) Purification using flash silica gel column chromatography (eluent: 10:1 hexane:EtOAc) gave the pure product as a colorless oil (40.2 $\mathrm{mg}, 72 \%$ yield).

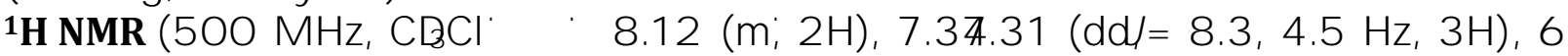
$(\mathrm{dd}, \mathrm{J}=8.4,2.5 \mathrm{~Hz}, 1 \mathrm{H}), 6.93(\mathrm{~d},=2.5 \mathrm{~Hz}, 1 \mathrm{H}), 4.27(\mathrm{~s}, 2 \mathrm{H}), 2.93(\mathrm{~d} d=9.7,4.9 \mathrm{~Hz}, 2 \mathrm{H})$, $2.82(\mathrm{~s}, 1 \mathrm{H}), 2.74(\mathrm{ttJ}=12.1,3.4 \mathrm{~Hz}, 1 \mathrm{H}), 2.50(\mathrm{dg},=19.0,8.6 \mathrm{~Hz}, 1 \mathrm{H}), 2.42 .40(\mathrm{~m}$, $1 \mathrm{H}), 2.31(\mathrm{td}, \mathrm{J}=10.8,4.0 \mathrm{~Hz}, 1 \mathrm{H}), 2.182 .11(\mathrm{~m}, 1 \mathrm{H}), 2.081 .95(\mathrm{~m}, 3 \mathrm{H}), 1.85(\mathrm{dJ}=12.8$ $\mathrm{Hz}, 2 \mathrm{H}), 1.70 \mathrm{z} 1.57(\mathrm{~m}, 4 \mathrm{H}), 1.56 \mathrm{z} 1.44(\mathrm{~m}, 4 \mathrm{H}), 1.49$ (s, 9H), 0.91 (s, 3H).C NMR (126 


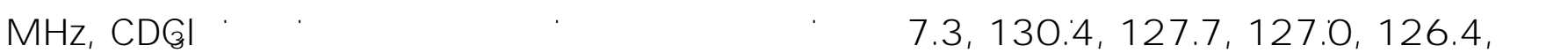
121.6, 118.8, 79.5, 50.4, 47.9, 44.1, 44.0, 42.8, 38.0, 35.8, 32.8, 31.5, 29.4, 28.4, 26.3, 25.7, 21.5, $13.8 \mathrm{ppm}$.

HRMS (ESI): Calcd. 580.3033 for $\mathrm{g}_{5} \mathrm{H}_{43} \mathrm{NNaO}_{5}+(\mathrm{M}+\mathrm{Na})^{+}$, found 580.3029 .

\section{tert -Butyl 4-(4-((( (3R,4S,5R,6B-3,4,5-tris(benzyloxy)-6-((benzyloxy)methyl)tetra} hydro-2H-pyran-2-yl)oxy)carbonyl)phenyl)piperidine-1-carboxylate (4n):

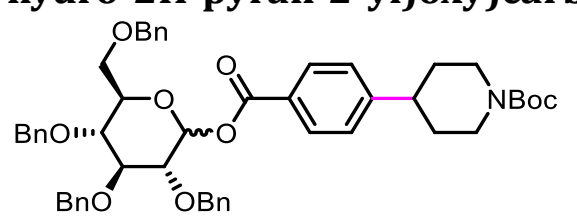

Following the GP2, the title compound was prepared from $(3 \mathrm{R}, 4 \mathrm{~S}, 5 \mathrm{R}, 6 \mathrm{R} 3,4,5$ tris(benzyloxy) -6-((benzyloxy)methyl)tetrahydro -2H-pyran-2-yl 4-bromobenzoate (73 $\mathrm{mg})$ andPy S1 (75.1 mg)P urification using flash silica gel column chromatography (eluent: 5:1 hexane:EtOAc) gave the pure product as a colorless $6 \mathrm{fiR}(9 \mathrm{mg}, 76 \%$ yield, : $1: 1)$

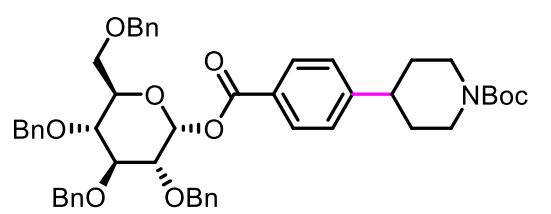

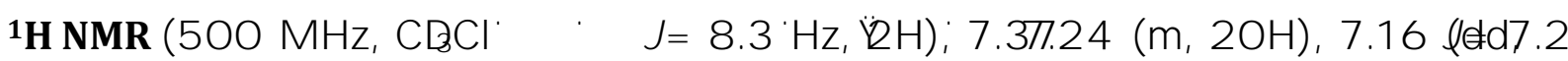
$2.1 \mathrm{~Hz}, 2 \mathrm{H}), 6.60(\mathrm{dJ}=3.5 \mathrm{~Hz}, 1 \mathrm{H}), 4.99(\mathrm{~d},=10.9 \mathrm{~Hz}, 1 \mathrm{H}), 4.88(\mathrm{~d},=6.7 \mathrm{~Hz}, 1 \mathrm{H}), 4.86$ $(\mathrm{d}, \mathrm{J}=7.1 \mathrm{~Hz}, 1 \mathrm{H}), 4.75(\mathrm{~d},=11.6 \mathrm{~Hz}, 1 \mathrm{H}), 4.66(\mathrm{~d},=11.6 \mathrm{~Hz}, 1 \mathrm{H}), 4.62(\mathrm{~d},=12.1 \mathrm{~Hz}$, $1 \mathrm{H}), 4.54(\mathrm{~d}, \mathrm{~J}=10.5 \mathrm{~Hz}, 1 \mathrm{H}), 4.48(\mathrm{~d},=12.1 \mathrm{~Hz}, 1 \mathrm{H}), 4.27(\mathrm{~s}, 2 \mathrm{H}), 4.06 \mathrm{gt}=9.3 \mathrm{~Hz}, 1 \mathrm{H})$, $3.99 \mathrm{z} 3.96(\mathrm{~m}, 1 \mathrm{H}), 3.84 \mathrm{z} 3.77(\mathrm{~m}, 3 \mathrm{H}), 3.65(\mathrm{ddJ}=10.9,1.8 \mathrm{~Hz}, 1 \mathrm{H}), 2.82(\mathrm{t} \mathrm{J}=11.1 \mathrm{~Hz}$, $1 \mathrm{H}), 2.73(\mathrm{tt}, \mathrm{J}=12.0,3.3 \mathrm{~Hz}, 1 \mathrm{H}), 1.83(\mathrm{~g},=12.7 \mathrm{~Hz}, 2 \mathrm{H}), 1.6 \mathrm{z} 1.62(\mathrm{~m}, 2 \mathrm{H}), 1.49(\mathrm{~s}$,

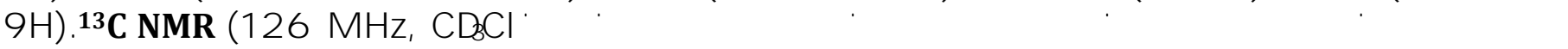
128.5, 128.4, 128.1, 128.1, 128.0, 127.927.8, 127.7, 127.6, 127.0, 90.5, 81.8, 79.6, 79.0, 77.0, 75.7, 75.5, 73.6, 73.1, 73.0, 68.1, 44.0, 42.8, 32.8, $28.5 \mathrm{ppm}$.

HRMS (ESI): Calcd. 850.3926 for ${ }_{4} \mathrm{H}_{57} \mathrm{NNaO}_{3}+(\mathrm{M}+\mathrm{Na})^{+}$, found 850.3916 .

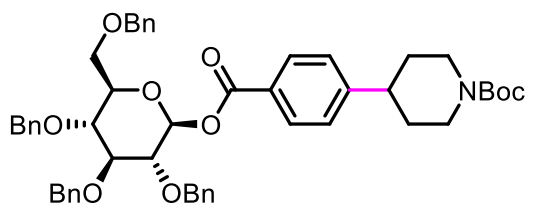

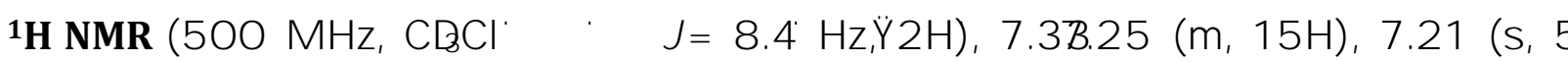
7.17 z $7.15(\mathrm{~m}, 2 \mathrm{H}), 5.88(\mathrm{dJ}=7.8 \mathrm{~Hz}, 1 \mathrm{H}), 4.91(\mathrm{~d},=11.0 \mathrm{~Hz}, 1 \mathrm{H}), 4.85(\mathrm{~d},=3.7 \mathrm{~Hz}$, $1 \mathrm{H}), 4.84 \mathrm{z} 4.82(\mathrm{~m}, 1 \mathrm{H}), 4.82(\mathrm{dJ}=11.1 \mathrm{~Hz}, 1 \mathrm{H}), 4.77(\mathrm{~g},=11.1 \mathrm{~Hz}, 1 \mathrm{H}), 4.61(\mathrm{~d},=12.1$ $\mathrm{Hz}, 1 \mathrm{H}), 4.56(\mathrm{dJ}=10.8 \mathrm{~Hz}, 1 \mathrm{H}), 4.48(\mathrm{~g},=12.1 \mathrm{~Hz}, 1 \mathrm{H}), 4.27(\mathrm{~s}, 2 \mathrm{H}), 3.843 .73(\mathrm{~m}, 5 \mathrm{H})$, 3.67 z $3.65(\mathrm{~m}, 1 \mathrm{H}), 2.82(\mathrm{tJ}=10.6 \mathrm{~Hz}, 2 \mathrm{H}), 2.72(\mathrm{ttJ}=12.1,3.4 \mathrm{~Hz}, 1 \mathrm{H}), 1.84(\mathrm{gl}=12.3$ $\mathrm{Hz}, 2 \mathrm{H}), 1.68 \mathrm{z} 1.63(\mathrm{~m}, 2 \mathrm{H}), 1.49(\mathrm{~s}, 9 \mathrm{H}) \mathbf{1}^{3} \mathbf{C} \mathbf{N M R}(126 \mathrm{MHz}, \mathrm{CDGQ}$ а J ЙЋ 8 y̆ Џh $151.8,138.4,138.0,137.9,137.8,130.4,128.4,128.3,128.0,127.9,127.9,127.8,127.8$, $127.7,127.7,127.6,127.4,126.9,94.6,84.9,80.9,79.6,77.3,75.7,75.6,75.0,73.5,68.1$, $44.4,42.8,32.8,29.7,28.5 \mathrm{ppm}$.

Methyl 4-cyclohexylbenzoate (4o) ${ }^{5}$ :

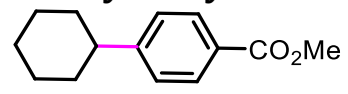


Following the GP2, the title compound was prepared from methyl 4bromobenzoate (21.5 $\mathrm{mg}$ ) andPy S2 (62 mg)Purification using flash silica gel column chromatography (eluent: 30:1 hexane:EtOAc) gave the pure product as a colorless olib( $4 \mathrm{mg}, 75 \%$ yield).

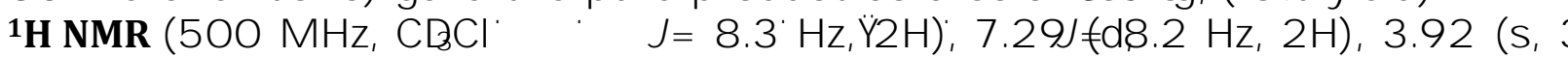
2.57 (ddd, J = 11.5, 7.3, 3.1 Hz, 1H), $1.901 .86(\mathrm{~m}, 4 \mathrm{H}), 1.80 \mathrm{z} 1.77(\mathrm{~m}, 1 \mathrm{H}), 1.49 \mathrm{z} 1.37$ (m, 4H), 1.33z 1.25 (m, 1H). ${ }^{13}$ C NMR (126 MHz, CDGQ I 2J1 в̈вЙ481297, 127.7, $126.8,519,44.7,34.1,26.7,26 . \phi p m$.

\section{1-Cyclohexyl-4-methoxybenzene (4p):<smiles>COc1ccc(C2CCCCC2)cc1</smiles>

Following the GP2, the title compound was prepared from 4methoxy-bromobenzene (19 $\mathrm{mg}$ ) and Py S2 (95 mg, $2.0 \mathrm{eq}$ ) using $20 \mathrm{~mol} \% \mathrm{Ni}$ and $25 \mathrm{~mol} \% \mathrm{~L}$, Purification using flash silica gel column chromatography (eluent30:1 hexane:EtOAc) gave the pure product as a colorless oil $6.5 \mathrm{mg}, 34 \%$ yield).

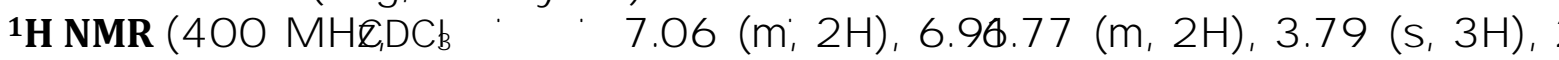
$2.38(\mathrm{~m}, 1 \mathrm{H}), 1.84(\mathrm{tg} J \mathrm{~J}=5.6,2.5 \mathrm{~Hz}, 4 \mathrm{H}), 1.7 \mathrm{Z} 1.68(\mathrm{~m}, 1 \mathrm{H}), 1.45 \mathrm{Z} 1.34(\mathrm{~m}, 4 \mathrm{H}), 1.29 \mathrm{Z}$

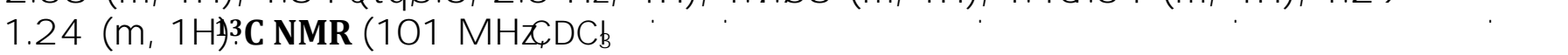
26.9, 26.2ppm.

HRMS (EI) for: $\mathrm{C}_{13} \mathrm{H}_{18} \mathrm{O}[\mathrm{M}]^{+}$: calcd190.1352, found 190.1357.

(4-Cyclohexylphenyl)(pyrrolidin-1-yl)methanone (4q):

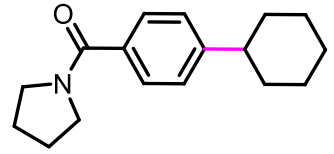

Following the GP2, the title compound was prepared from(4-bromophenyl)(pyrrolidin 1-yl)methanone (25.5 mg) andPy S2 (62 mg),Purification using flash silica gel column chromatography (eluent:2:1 hexane:EtOAc) gave the pure product as a colorless diB(8 $\mathrm{mg}, 73 \%$ yield).

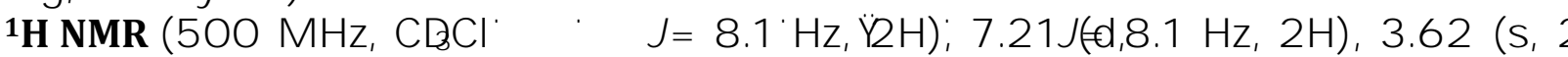
$3.45(\mathrm{~s}, 2 \mathrm{H}), 2.52 \mathrm{z} 2.48(\mathrm{~m}, 1 \mathrm{H}), 1.93 \mathrm{z} 1.83(\mathrm{~m}, 8 \mathrm{H}), 1.74(\mathrm{dJ}=12.6 \mathrm{~Hz}, 1 \mathrm{H}), 1.4$ 建 1.33 $(\mathrm{m}, 4 \mathrm{H}), 1.28 \mathrm{z} 1.23(\mathrm{~m}, 1 \mathrm{H}) .{ }^{13} \mathbf{C}$ NMR $\left(126 \mathrm{MHz}, \mathrm{CDG}_{8} \mathrm{l}\right.$ J Й $8 \mathrm{~A} \mathrm{~h}$ J Ћ A 8 A h̆ 126.6, 49.6, 46.2, 44.4, 34.2, 26.8, 26.4, Q,624.4 ppm.

HRMS (EI): Calcd. 256.1696 for $\mathrm{GH}_{22} \mathrm{NO}^{+}[\mathrm{M}-\mathrm{H}]+$, found 256.1700 .

\section{4-Cyclohexylpicolinonitrile (4r):}

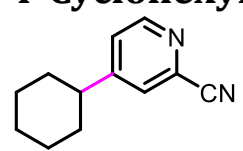

Following the GP2, the title compound was prepared from 4bromopicolinonitrile (18.3 $\mathrm{mg}$ ) and Py S2 (62 mg), Purification using flash silica gel column chromatography (eluent: $5: 1$ hexane:EtOAc) gave the pure product as a colorless diB( $8 \mathrm{mg}, 74 \%$ yield).

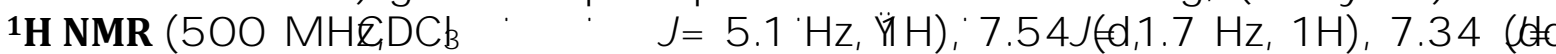
$5.2,1.7 \mathrm{~Hz}, 1 \mathrm{H}), 2.56(\mathrm{df},=9.9,6.4 \mathrm{~Hz}, 1 \mathrm{H}), 1.89(\mathrm{dd}=7.2,4.6 \mathrm{~Hz}, 4 \mathrm{H}), 1.821 .74(\mathrm{~m}$, 1H), $1.46 \mathrm{z} 1.34(\mathrm{~m}, 4 \mathrm{H}), 1.29 \mathrm{z} 1.23(\mathrm{~m}, 1 \mathrm{H}){ }^{13} \mathbf{C} \mathbf{~ N M R}(101 \mathrm{MHz}, \mathrm{CDG} \mathrm{Q}$ 1 J150. $133.9,127.3,125.6,117.5,43.5,33.3,26.3,25.6$.

HRMS (EI) for: $\mathrm{C}_{12} \mathrm{H}_{14} \mathrm{~N}_{2}[\mathrm{M}]^{+}$: calcd186.1151, found 186.1158 . 


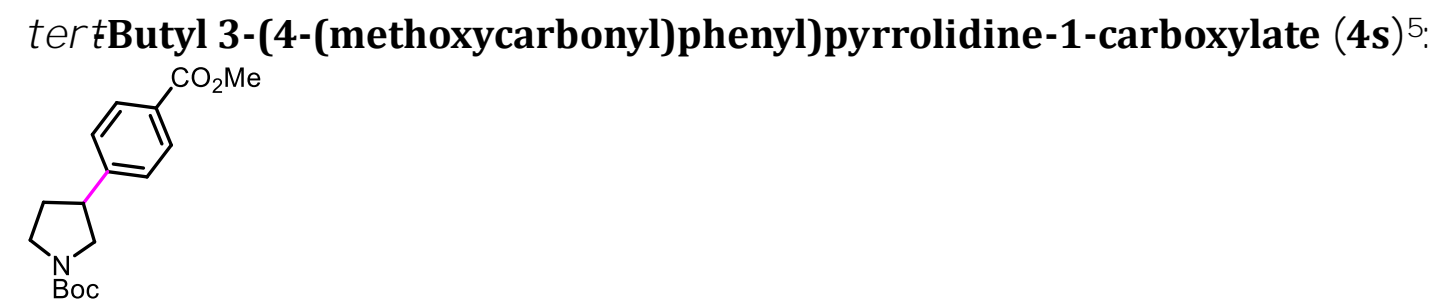

Following the GP2, the title compound was prepared from methyl 4bromobenzoate (21.5 $\mathrm{mg}$ ) andPy S4 (72 mg)Purification using flash silica gel column chromatography (eluent: 10:1 hexane:EtOAc) gave the pure product as a colorless $d i b(6 \mathrm{mg}, 51 \%$ yield). rotator isomer $(1: 1)$

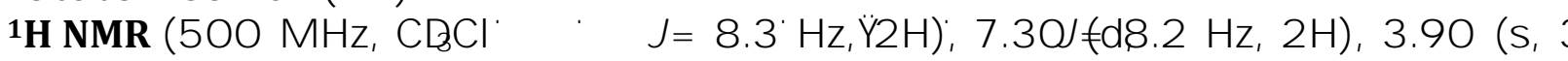
3.87 z $3.76(\mathrm{~m}, 1 \mathrm{H}), 3.66 \mathrm{z} 3.54(\mathrm{~m}, 1 \mathrm{H}), 3.4 \mathrm{z} 3.27(\mathrm{~m}, 3 \mathrm{H}), 2.28 \mathrm{z} 2.26(\mathrm{~m}, 1 \mathrm{H}), 1.99(\mathrm{dt}$,

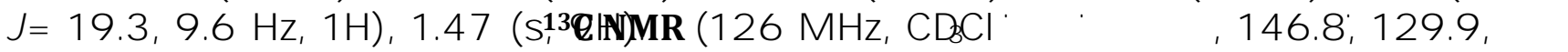
128.5, 127.1, 79.4, 52.2, 52.1, 51.5, 45.8, 45.5, 44.2, 43.3, 33.2, 32.3, 28.5 ppm.<smiles>CC(CCc1ccccc1)c1ccc(C(F)(F)F)cc1</smiles>

Following the GP2, the title compound was prepared from1-bromo-4-(trifluoromethyl) benzene (22.5 mg) and Py S3 (69 mg),Purification using flash silica gel column chromatography (eluent: 50:1 hexane:EtOAc) gave the pure product as a colorless oil (24.5 mg, 88\% yield).

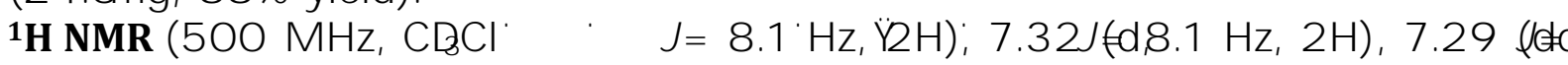
10.4, 4.6 Hz, 2H), $7.19(\mathbb{H},=7.4 \mathrm{~Hz}, 1 \mathrm{H}), 7.14(\mathrm{~d} J,=7.1 \mathrm{~Hz}, 2 \mathrm{H}), 2.8<2.77(\mathrm{~m}, 1 \mathrm{H}), 2.58$ z $2.48(\mathrm{~m}, 2 \mathrm{H}), 2.00 \mathrm{z} 1.90(\mathrm{~m}, 2 \mathrm{H}), 1.30(\mathrm{dJ}=7.0 \mathrm{~Hz}, 3 \mathrm{H}){ }^{\mathbf{1}} \mathbf{F}$ NMR $\left(471 \mathrm{MHz}, \mathrm{CDG}_{3} \mathrm{Q}\right.$ - $\mathrm{I}$

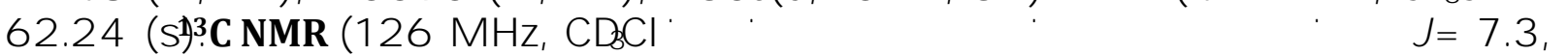
$3.6 \mathrm{~Hz}), 124.4(\mathrm{q} J=271.9 \mathrm{~Hz}) 39.7,39.4,33.8,22.2 \mathrm{ppm}$.

\section{2-Chloro-1-fluoro-4-(4-phenylbutan-2-yl)benzene (4u):}<smiles>CC(CCc1ccccc1)c1ccc(F)c(Cl)c1</smiles>

Following the GP2, the title compound was prepared from 4-bromo-2-chloro-1fluorobenzene $(21.0 \mathrm{mg}$ ) and Py S3 (69 mg),Purification using flash silica gel column chromatography (eluent: 50:1 hexane:EtOAc) gave the pure product as a colorless oil (18.9 mg, $72 \%$ yield).

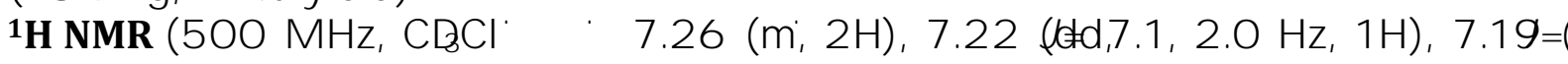
$7.3 \mathrm{~Hz}, 1 \mathrm{H}), 7.13(\mathrm{dJ}=7.0 \mathrm{~Hz}, 2 \mathrm{H}), 7.027 .03(\mathrm{~m}, 1 \mathrm{H}), 2.73 \mathrm{z} 2.66(\mathrm{~m}, 1 \mathrm{H}), 2.56 \mathrm{z} 2.47$ $(\mathrm{m}, 2 \mathrm{H}), 1.91 \mathrm{z} 1.86(\mathrm{~m}, 2 \mathrm{H}), 1.26(\mathrm{dJ}=7.0 \mathrm{~Hz}, 3 \mathrm{H}){ }^{\mathbf{1}} \mathbf{F} \mathbf{~ N M R}(471 \mathrm{MHz}, \mathrm{CDC} \mathrm{O}$ - 1119.78 (s). ${ }^{13}$ C NMR (126 MHz, CDGQ I J h́ 128.3, 128.3, 126.6 (dJ = 6.8 Hz), 125.8, $116.4(\mathrm{~d},=20.7 \mathrm{~Hz}), 39.8,38.7,33.7,22.4 \mathrm{ppm}$. HRMS (EI): Calcd. 262.0919 for ${ }_{66} \mathrm{H}_{16} \mathrm{ClF}^{+}[\mathrm{M}]+$, found 262.0916. 
Morpholino(4-(4-phenylbutan-2-yl)phenyl)methanone (4v):<smiles>CC(CCc1ccccc1)c1ccc(C(=O)N2CCOCC2)cc1</smiles>

Following the GP2, the title compound was prepared from (4-bromophenyl) (morpholino)methanone (27 mg) and Py S3 (69 mg),Purification using flash silica gel column chromatography (eluent:2:1 hexane:EtOAc) gave the pure product as a colorless oil ( $27.8 \mathrm{mg}, 86 \%$ yield).

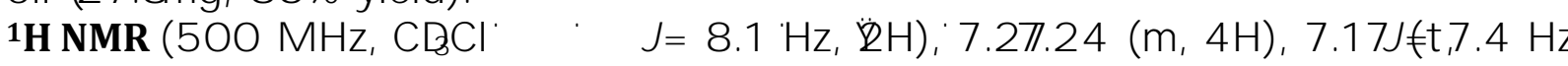
$1 \mathrm{H}), 7.12(\mathrm{~d}, \mathrm{~J}=7.1 \mathrm{~Hz}, 2 \mathrm{H}), 3.7 \mathrm{z} .52(\mathrm{~m}, 8 \mathrm{H}), 2.79 \mathrm{z} 2.72(\mathrm{~m}, 1 \mathrm{H}), 2.56 \mathrm{z} 2.46(\mathrm{~m}, 2 \mathrm{H})$,

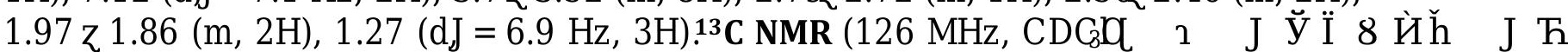
142.1, 132.9, 128.3, 127.3, 127.2, 125.7, 66.9, 48.3, 42.6, 39.7, 39.4, 33.8, 22.3 ppm.

HRMS (EI): Calcd. 322.1802 for $\mathrm{E}_{1} \mathrm{H}_{24} \mathrm{NO}_{2}+[\mathrm{M}-\mathrm{H}]^{+}$, found 322.1806 .

(4-(Heptan-2-yl)phenyl)(morpholino)methanone (4w):<smiles>CCCCC(C)c1ccc(C(=O)N2CCOCC2)cc1</smiles>

Following the GP2, the title compound was prepared from (4-bromophenyl) (morpholino)methanone $(27 \mathrm{mg}$ ) and Py S10 (74 mg),Purification using flash silica gel column chromatography (eluent:2:1 hexane:EtOAc) gave the pure product as a colorless oil ( $23.7 \mathrm{mg}, 82 \%$ yield).

1H NMR (500 MHz, CDGQ I $(\mathrm{m}, 8 \mathrm{H}), 2.69(\mathrm{hJ}=7.0 \mathrm{~Hz}, 1 \mathrm{H}), 1.5 \mathrm{q} 1.52(\mathrm{~m}, 2 \mathrm{H}), 1.26 \mathrm{z} 1.12(\mathrm{~m}, 9 \mathrm{H}), 0.83(\mathrm{tJ}=6.8$

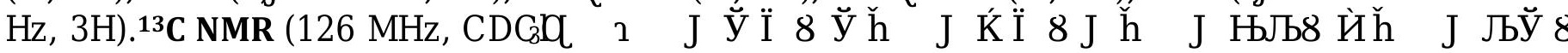
$39.8,38.2,31.8,27.2,22.5,22.1,14.0 \mathrm{ppm}$.

HRMS (EI): Calcd. 288.1958 for $\mathrm{C}_{8} \mathrm{H}_{26} \mathrm{NO}_{2}+[\mathrm{M}-\mathrm{H}]^{+}$, found 288.1952.

(4-(3-Methylbutan-2-yl)phenyl)(morpholino)methanone (4x):<smiles>CC(C)C(C)c1ccc(C(=O)N2CCOCC2)cc1</smiles>

Following the GP2, the title compound was prepared (4-bromophenyl) (morpholino)methanone (27 mg) and Py S5 (61 mg),Purification using flash silica gel column chromatography (eluent:3:1 hexane:EtOAc) gave the pure product as a colorless oil (19.6 mg, $75 \%$ yield).

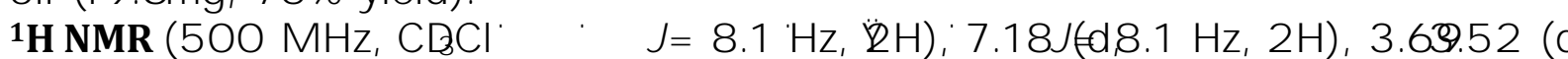
$\mathrm{J}=87.7 \mathrm{~Hz}, 8 \mathrm{H}), 2.44(\mathrm{pJ}=7.1 \mathrm{~Hz}, 1 \mathrm{H}), 1.721 .72(\mathrm{~m}, 1 \mathrm{H}), 1.22(\mathrm{dJ}=7.0 \mathrm{~Hz}, 3 \mathrm{H}), 0.92$ $(\mathrm{d}, \mathrm{J}=6.7 \mathrm{~Hz}, 3 \mathrm{H}), 0.74(\mathrm{~d},=6.7 \mathrm{~Hz}, 3 \mathrm{H}) \mathbf{1}^{\mathbf{3}} \mathbf{C} \mathbf{N M R}(126 \mathrm{MHz}, \mathrm{CDG} \mathrm{Q} \quad$ I $\quad$ J $127.8,127.0,66.9,48.2,46.7,42.6,34.3,21.1,20.1,18.6 \mathrm{ppm}$.

HRMS (EI): Calcd. 260.1645 for ${ }_{6} \mathrm{H}_{22} \mathrm{NO}_{2}+[\mathrm{M}-\mathrm{H}]^{+}$, found 260.1644 .

(4-(1-Cyclohexylethyl)phenyl)(morpholino)methanone (4y): 
<smiles>CC(c1ccc(C(=O)N2CCOCC2)cc1)C1CCCCC1</smiles>

Following the GP2, the title compound was prepared from (4-bromophenyl) (morpholino)methanone (27 mg) and Py S6 (66 mg) Purification using flash silica gel column chromatography (eluent:3:1 hexane:EtOAc) gave the pure product as a colorless oil ( $23.5 \mathrm{mg}, 78 \%$ yield).

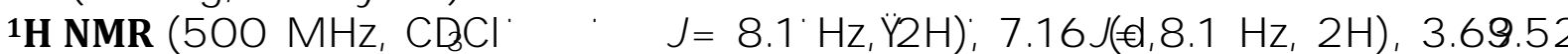
$(\mathrm{m}, 8 \mathrm{H}), 2.46(\mathrm{pJ}=7.2 \mathrm{~Hz}, 1 \mathrm{H}), 1.85(\mathrm{dJ}=12.7 \mathrm{~Hz}, 1 \mathrm{H}), 1.72(\mathrm{dd},=10.4,2.9 \mathrm{~Hz}, 1 \mathrm{H})$, $1.61 \mathrm{z} 1.59(\mathrm{~m}, 2 \mathrm{H}), 1.42 \mathrm{z} 1.35(\mathrm{~m}, 2 \mathrm{H}), 1.21(\mathrm{dJ}=7.0 \mathrm{~Hz}, 3 \mathrm{H}), 1.231 .16(\mathrm{~m}, 1 \mathrm{H}), 1.13$ z $1.03(\mathrm{~m}, 2 \mathrm{H}), 0.92$ (dddJ = 24.2, 12.5, 3.5 Hz, 1H), 0.830.73 (m, 1H). ${ }^{13}$ C NMR (126

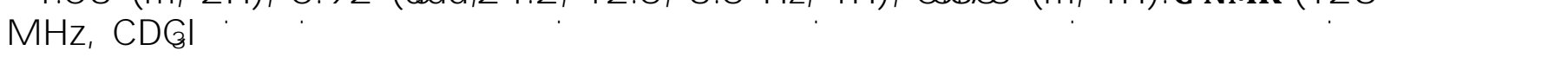
$26.4,26.4,18.6 \mathrm{ppm}$.

HRMS (EI): Calcd. 300.1958 for ${ }_{69} \mathrm{H}_{26} \mathrm{NO}_{2}+[\mathrm{M}-\mathrm{H}]^{+}$, found 300.1962 .

(4-(1-Hydroxybutan-2-yl)phenyl)(morpholino)methanone (4z):<smiles>CCC(CO)c1ccc(C(=O)N2CCOCC2)cc1</smiles>

Following the GP2, the title compound was prepared from(4-bromophenyl) (morpholino)methanone (27 mg) and Py S8 (61 mg), Purification using flash silica gel column chromatography (eluent:1:2 hexane:EtOAc) gave the pure product as a colorless oil (10.5 mg, 40\% yield).

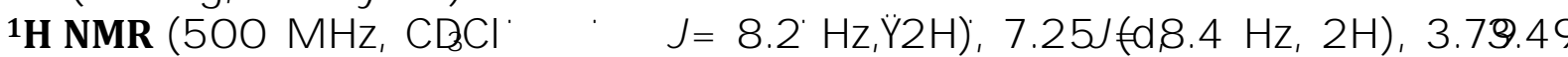
$(\mathrm{m}, 10 \mathrm{H}), 2.72(\mathrm{ddt} J=9.3,7.7,5.6 \mathrm{~Hz}, 1 \mathrm{H}), 1.821 .73(\mathrm{~m}, 1 \mathrm{H}), 1.62 z 1.53(\mathrm{~m}, 2 \mathrm{H}), 0.83$

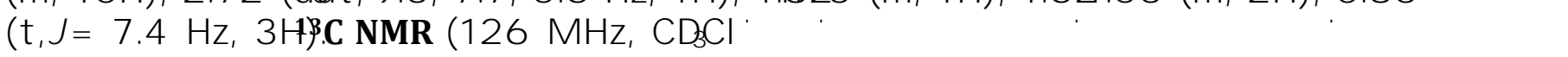
$66.9,50.3,48.2,42.6,24.9,11.9 \mathrm{ppm}$.

HRMS (ESI): Calcd. 286.1414 for ${ }_{5} \mathrm{H}_{21} \mathrm{NNaO}_{3}{ }^{+}(\mathrm{M}+\mathrm{Na})^{+}$, found 286.1419 .

(4-(1-(2,6-Dimethylphenoxy)propan-2-yl)phenyl)(morpholino)methanone (4aa):<smiles>Cc1cccc([N+](=O)[O-])c1OCC(c1ccc(C(=O)N2CCOCC2)cc1)[N+](=O)[O-]</smiles>

Following the GP2, the title compound was prepared from (4-bromophenyl) (morpholino)methanone (27 mg) and Py S11 (72 mg) Purification using flash silica gel column chromatography (eluent:2:1 hexane:EtOAc) gave the pure product as a colorless oil (21.6 mg, 61\% yield).

1H NMR (500 MHz, CDGQ a $1 \mathrm{H}), 3.85 \mathrm{z} 3.79(\mathrm{~m}, 2 \mathrm{H}), 3.73 \mathrm{z} 3.48(\mathrm{~m}, 8 \mathrm{H}), 3.32 \mathrm{z} 3.25(\mathrm{~m}, 1 \mathrm{H}), 2.14(\mathrm{~s}, 6 \mathrm{H}), 1.46(\mathrm{~d}=$

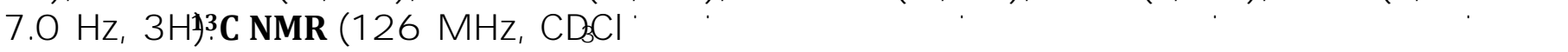
127.3, 123.8, 66.9, 48.3, 48, 40.6, 18.0, $16.1 \mathrm{ppm}$.

HRMS (EI): Calcd. 353.1985 for $6_{2} \mathrm{H}_{27} \mathrm{NO}_{3}{ }^{+}[\mathrm{M}]+$, found 353.1988 . 
2-(4-(Morpholine-4-carbonyl)phenyl)butyl 2-(1-(4-chlorobenzoyl)-5-methoxy-2methyl -1H-indol-3-yl)acetate (4ab):<smiles>CCC(COC(=O)Cc1c(C)n(C(=O)c2ccc(Cl)cc2)c2ccc(OC)cc12)c1ccc(C(=O)N2CCOCC2)cc1</smiles>

Following the GP2, the title compound was prepared from (4-bromophenyl) (morpholino)methanone (27 mg) and Py S13 (105 mg) Purification using flash silica gel column chromatography (eluent:2:1 hexane:EtOAc) gave the pure product as a colorless oil (33.8 mg, 56\% yield).

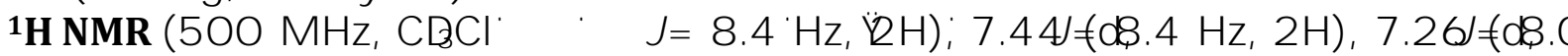
$\mathrm{Hz}, 2 \mathrm{H}), 7.07(\mathrm{dJ}=8.1 \mathrm{~Hz}, 2 \mathrm{H}), 6.89(\mathrm{dJ}=2.5 \mathrm{~Hz}, 1 \mathrm{H}), 6.85(\mathrm{~d},=9.0 \mathrm{~Hz}, 1 \mathrm{H}), 6.65$ (dd, $=9.0,2.5 \mathrm{~Hz}, 1 \mathrm{H}), 4.2 \mathrm{Z} 4.19(\mathrm{~m}, 2 \mathrm{H}), 3.80(\mathrm{~s}, 3 \mathrm{H}), 3.783 .40(\mathrm{~m}, 8 \mathrm{H}), 3.57(\mathrm{~s}, 2 \mathrm{H}), 2.77$ $(\mathrm{dq}, \mathrm{J}=9.3,6.4 \mathrm{~Hz}, 1 \mathrm{H}), 2.26(\mathrm{~s}, 3 \mathrm{H}), 1.67(\mathrm{dd} d \mathrm{~d}=14.5,10.1,6.6 \mathrm{~Hz}, 1 \mathrm{H}), 1.571 .48(\mathrm{~m}$,

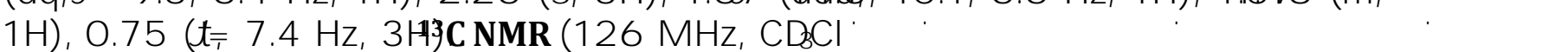
139.2 , 135.8, 133.8, 133.6, 131.1129.1, 128.5, 128.0, 127.3, 127.0, 68.3, 66.8, 55.7, 48.2, $46.5,42.6,30.3,25.1,13.2,11.7 \mathrm{ppm}$.

HRMS (ESI): Calcd. 625.2076 for ${ }_{4} \mathrm{H}_{35} \mathrm{ClN}_{2} \mathrm{NaO}_{6}+(\mathrm{M}+\mathrm{Na})^{+}$, found 625.2073.

4-((4-phenethylphenyl)sulfonyl)morpholine (4ac):<smiles>O=S(=O)(c1ccc(CCc2ccccc2)cc1)N1CCOCC1</smiles>

Following the GP2, the title compound was prepared from 4((4-bromophenyl)sulfonyl) morpholine (30.6 mg) and Py S14 (75 mg, 1.5 equiv) using $12 \mathrm{~mol} \%$-tHu-bpy as ligand and 3.0 equiv TEA as additive, Purification using flash silica gel column chromatography (eluent: 3:1 hexane:EtOAc) gave the pure product as a colorless 0 lib(9 mg, 51\% yield).

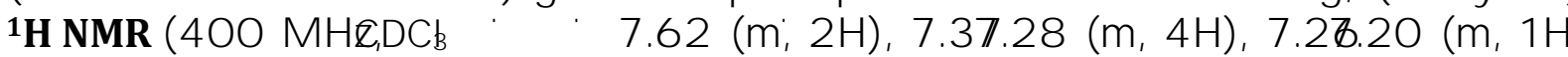
7.19 z $7.11(\mathrm{~m}, 2 \mathrm{H}), 3.86 \mathrm{z} 3.69(\mathrm{~m}, 4 \mathrm{H}), 3.15 \mathrm{z} 2.87(\mathrm{~m}, 8 \mathrm{H}) .{ }^{13} \mathbf{C}$ NMR $(101 \mathrm{MHz}, \mathrm{CDG})$

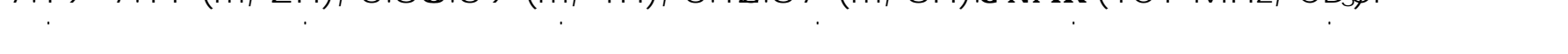
HRMS (EI) for: $\mathrm{C}_{18} \mathrm{H}_{21} \mathrm{NO}_{3} \mathrm{~S}$ [M]': calcd 331.1237, found 331.1235.

4-((4-(2-(Benzo[d][1,3]dioxol-5-yl)ethyl)phenyl)sulfonyl)morpholine (4ad):<smiles>O=S(=O)(c1ccc(CCc2ccc3c(c2)OCO3)cc1)N1CCOCC1</smiles>

Following the GP2, the title compound was prepared from 4((4-bromophenyl)sulfonyl) morpholine (30.6 mg) and Py S17 (81 mg, 1.5 equiv) using $12 \mathrm{~mol} \%$-t and 3.0 equiv TEA as additive, Purification using flash silica gel column chromatography (eluent: 3:1 hexane:EtOAc) gave the pure product as a white solid8.8 mg, 50\% yield).

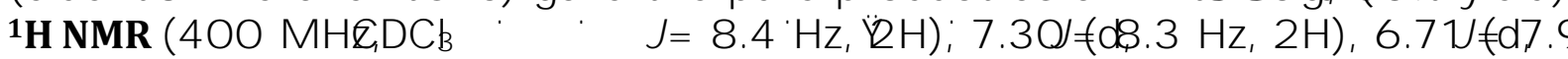
$\mathrm{Hz}, 1 \mathrm{H}), 6.62(\mathrm{dJ}=1.7 \mathrm{~Hz}, 1 \mathrm{H}), 6.55(\mathrm{dd},=7.9,1.7 \mathrm{~Hz}, 1 \mathrm{H}), 5.93(\mathrm{~s}, 2 \mathrm{H}), 3 . \& 03.69(\mathrm{~m}$, 
4H), 3.07z $2.91(\mathrm{~m}, 6 \mathrm{H}), 2.90 \mathrm{z} 2.80(\mathrm{~m}, 2 \mathrm{H}) .{ }^{13} \mathbf{C}$ NMR $(101 \mathrm{MHz}, \mathrm{CDG} \mathrm{q} \quad$ 1 J Ћ Ћ $145.9,134.4,132.6,129.2,127.9,121.2,108.8,108.2,100.9,66.1,46.0,37.9, \mathrm{pppm0}$

HRMS (EI) for: C19H21NO5S [M]+: calcd375.1135, found 375.1133.

tert-butyl 2-((4R,6R)-2,2-dimethyl-6-(4-(morpholine-4-carbonyl)phenethyl)-1,3dioxan-4-yl)acetate (4ae):<smiles>CC1(C)OC(COC(=O)OCc2ccccc2)CC(Cc2ccc(C(=O)N3CCOCC3)cc2)O1</smiles>

Following the GP2, the title compound was prepared from (4bromophenyl) (morpholino)methanone (27 mg) and Py S15 (98 mg, 1.équiv) using $12 \mathrm{~mol} \%$ ditBubpy as ligand and 3.0 equiv TEA as additive, Purification using flash silica gel column chromatography (eluent:2:1 hexane:EtOAc) gave the pure product as a colorless $\alpha \mathbb{\alpha}(7$ $\mathrm{mg}, 58 \%$ yield).

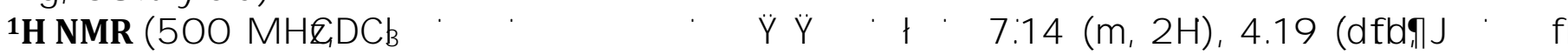
$=11.5,6.6,2.5 \mathrm{~Hz}, 1 \mathrm{H}), 3.873 .42(\mathrm{~m}, 9 \mathrm{H}), 2.82 \mathrm{z} 2.60(\mathrm{~m}, 2 \mathrm{H}), 2.41(\mathrm{dd}, \mathrm{J}=15.1,6.9 \mathrm{~Hz}$, 1H), $2.27(\mathrm{dt}, \mathrm{J}=15.1,4.8 \mathrm{~Hz}, 1 \mathrm{H}), 1.83 .75(\mathrm{~m}, 1 \mathrm{H}), 1.72 \mathrm{z} 1.61(\mathrm{~m}, 1 \mathrm{H}), 1.51(\mathrm{dtJ}=$ 12.8, 2.5 Hz, 1H), $1.42(\mathrm{~s}, 9 \mathrm{H}), 1.40(\mathrm{~s}, 3 \mathrm{H}), 1.37$ (s, 3H), 1.2416 (m, 1H). ${ }^{13} \mathbf{C}$ NMR (126 MHz, CDG.) 170.6, 170.3, 144.2, 132.7, 128.7, 127.3, 98.7, 80.6, 67.5, 66.9, 66.2, 42.7, 37.6, 36.5, 30.9, 30.1, 28.1, 19.8 .

HRMS (ESI) for: ${ }_{6} \mathrm{H}_{37} \mathrm{NO}_{6} \mathrm{Na}[\mathrm{M}+\mathrm{Na}]^{+}$: calcd470.2513, found 470.2509.

(4-(((1S,2R,59-6,6-Dimethylbicyclo[3.1.1]heptan-2-yl)methyl)phenyl) (morph olino)methanone (4af):<smiles>CC1(C)CCCC1Cc1ccc(C(=O)N2CCOCC2)cc1</smiles>

Following the GP2, the title compound was prepared from (4bromophenyl) (morpholino)methanone (27 mg) and Py S16 (80 mg, 1.5 equiv) using $12 \mathrm{~mol} \%$ - $\mathrm{EBu}$ bpy as ligand and 3.0 equiv TEA as additive, Purification using flash silica gel column chromatography (eluent:2:1 hexane:EtOAc) gave the pure product as a colorless diB(7 $\mathrm{mg}, 42 \%$ yield).

1H NMR (500 MHz,CDCłq a $93.0 \mathrm{~Hz}, 8 \mathrm{H}), 2.7 \mathrm{z} 2.63(\mathrm{~m}, 2 \mathrm{H}), 2.30$ (ddt, J = 12.3, 8.3, $4.1 \mathrm{~Hz}, 2 \mathrm{H}), 1 \mathrm{z} 98899(\mathrm{~m}, 2 \mathrm{H})$, $1.88 \mathrm{z} 1.79(\mathrm{~m}, 3 \mathrm{H}), 1.57 \mathrm{z} 1.47(\mathrm{~m}, 1 \mathrm{H}), 1.19(\mathrm{~s} 3 \mathrm{H}), 1.13(\mathrm{~s}, 3 \mathrm{H}), 0.84(\mathrm{~d}, \mathrm{~J}=9.6 \mathrm{~Hz}, 1 \mathrm{H})$. ${ }^{13}$ C NMR (126 MHz, CDGQ $38.7,33.7,29.7,28.2,26.4,23.5,22 \mathrm{ppm}$.

HRMS (EI) for: $\mathrm{C}_{21} \mathrm{H}_{29} \mathrm{NO}_{2}[\mathrm{M}]+$ : calcd327.2193, found 327.2204

2-(5-Methyl-7-phenylheptyl)isoindoline-1,3-dione (4b): 


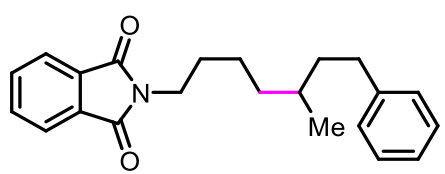

Following the GP3, the title compound was prepared from2-(4-iodobutyl)isoindoline 1,3-dione (33 mg) and Py S3 (79 mg,) Purification using flash silica gel column chromatography (eluent: 15:1 hexane:EtOAc) gave the pure product as a colorless oil (25.4 mg, 76\% yield).

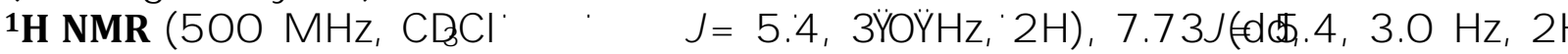
7.30 z $7.27(\mathrm{~m}, 2 \mathrm{H}), 7.20 \mathrm{z} 7.17(\mathrm{~m}, 3 \mathrm{H}), 3.70(\mathrm{t} J=7.3 \mathrm{~Hz}, 2 \mathrm{H}), 2.66$ (ddd, = 15.5, 10.6, $5.1 \mathrm{~Hz}, 1 \mathrm{H}), 2.57(\mathrm{dddJ}=13.7,9.9,6.1 \mathrm{~Hz}, 1 \mathrm{H}), 1.721 .60(\mathrm{~m}, 3 \mathrm{H}), 1.47 \mathrm{z} 1.21(\mathrm{~m}, 6 \mathrm{H})$,

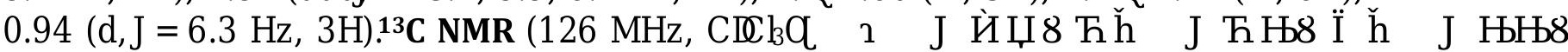
$128.2,125.5,123.1,38.8,38.0,36.4,33.4,32.3,28.9,24.2,19.5 \mathrm{ppm}$.

HRMS (EI): Calcd. 335.1880 for ${ }_{22} \mathrm{H}_{25} \mathrm{NO}_{2}+[\mathrm{M}]+$, found 335.1879 .

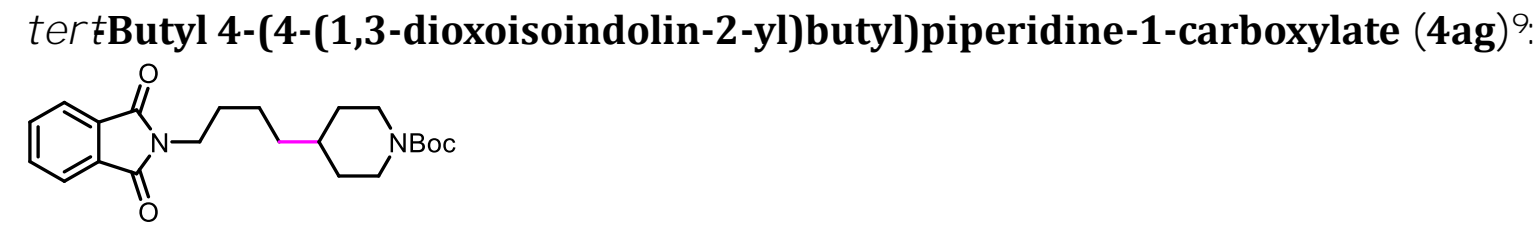

Following the GP3, the title compound was prepared from2-(4-iodobutyl)isoindoline 1,3-dione (33 mg) and Py S1 (87 mg) Purification using flash silica gel column chromatography (eluent: 6:1 hexane:EtOAc) gave theure product as a colorless oil 17.8 mg, $46 \%$ yield).

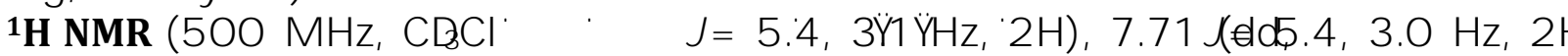
$4.04(\mathrm{~d}, \mathrm{~J}=11.0 \mathrm{~Hz}, 2 \mathrm{H}), 3.67(\mathrm{t}, \mathrm{J}=7.2 \mathrm{~Hz}, 2 \mathrm{H}), 2.64(\mathrm{t}, \mathrm{J}=12.3 \mathrm{~Hz}, 2 \mathrm{H}), 1.69 \mathrm{z} 1.63(\mathrm{~m}$, $4 \mathrm{H}), 1.44(\mathrm{~s}, 9 \mathrm{H}), 1.38 \mathrm{z} 1.32(\mathrm{~m}, 3 \mathrm{H}), 1.30 \mathrm{z} 1.25(\mathrm{~m}, 2 \mathrm{H}), 1.05$ (dddJ = 16.3, 12.6, $4.3 \mathrm{~Hz}$, 2H). ${ }^{13}$ C NMR (126 MHz, CDGQ I J Й $35.9,32.1,28.8,28.5,23.9 \mathrm{ppm}$.

tert -Butyl 4-(4-((2-oxo-2H-chromen-7-yl)oxy)butyl)piperidine-1-carboxylate (4ah):

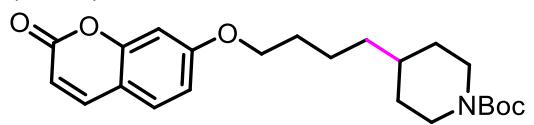

Following the GP3, the title compound was prepared from 7-(4-iodobutoxy)-2Hchromen-2-one (33.4 mg) and Py S1 (87mg) Purification using flash silica gel column chromatography (eluent:4:1 hexane:EtOAc) gave the pure product as a colorless diB(9 $\mathrm{mg}, 47 \%$ yield).

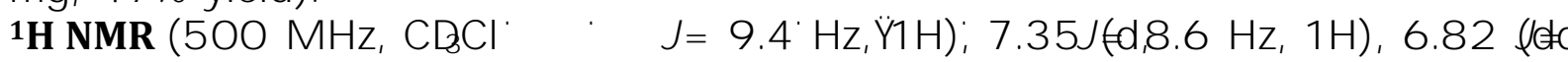
8.6, $2.4 \mathrm{~Hz}, 1 \mathrm{H}), 6.78(\mathrm{~g},=2.4 \mathrm{~Hz}, 1 \mathrm{H}), 6.23(\mathrm{~d},=9.5 \mathrm{~Hz}, 1 \mathrm{H}), 4.06(\mathrm{~s}, 2 \mathrm{H}), 4.00 \mathrm{gt}=6.4$ $\mathrm{Hz}, 2 \mathrm{H}), 2.66(\mathrm{~s}, 2 \mathrm{H}), 1.8 \mathrm{z} 1.77(\mathrm{~m}, 2 \mathrm{H}), 1.65(\mathrm{dJ}=12.7 \mathrm{~Hz}, 2 \mathrm{H}), 1.521 .47(\mathrm{~m}, 2 \mathrm{H})$, $1.44(\mathrm{~s}, 9 \mathrm{H}), 1.4 \mathrm{z} 1.37(\mathrm{~m}, 1 \mathrm{H}), 1.32 \mathrm{z} 1.27(\mathrm{~m}, 2 \mathrm{H}), 1.08(\mathrm{dddJ}=16.4,12.6,4.3 \mathrm{~Hz}, 2 \mathrm{H})$.

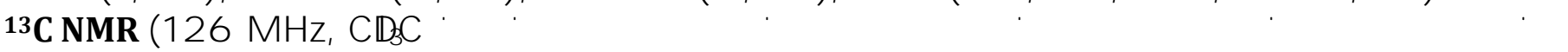
$101.3,79.2,68.5,44.0,36.2,35.9,32.1,29.1,28.4,23.0$ ppm.

HRMS (ESI): Calcd. 424.2093 for ${ }_{3} \mathrm{H}_{31} \mathrm{NNaO}_{5}{ }^{+}(\mathrm{M}+\mathrm{Na})^{+}$, found 424.2094.

2-(4-Cyclopentylbutyl)isoindoline-1,3-dione (4ai): 


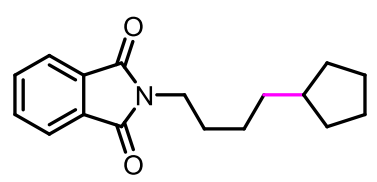

Following the GP3, the title compound was prepared from2-(4-iodobutyl)isoindoline 1,3-dione (33 mg) and Py S7 (70 mg) Purification using flash silica gel column chromatography (eluent: 15:1 hexane:EtOAc) gave the pumeroduct as a colorless oil (14.4 mg, 53\% yield).

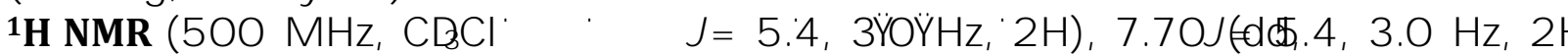
3.68 z $3.65(\mathrm{~m}, 2 \mathrm{H}), 1.73 \mathrm{z} 1.69(\mathrm{~m}, 3 \mathrm{H}), 1.68 \mathrm{z} 1.65(\mathrm{~m}, 2 \mathrm{H}), 1.60 \mathrm{z} 1.53(\mathrm{~m}, 2 \mathrm{H}), 1.52 \mathrm{z}$

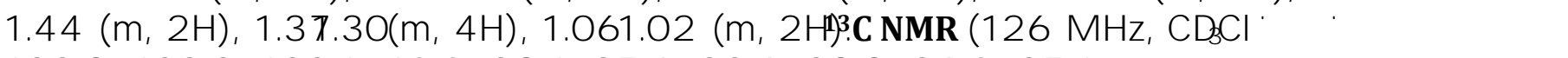
133.8, 132.2, 123.1, 40.0, 38.1, 35.6, 32.6, 28.8, 26.0, $25.1 \mathrm{ppm}$.

HRMS (EI): Calcd. 271.1567 for $\mathrm{GH}_{21} \mathrm{NO}_{2}+[\mathrm{M}]^{+}$, found 271.1562 .

\section{2-(4-Cycloheptylbutyl)isoindoline-1,3-dione (4aj):}

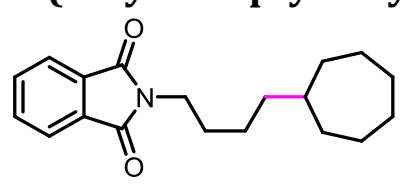

Following the GP3, the title compound was prepared from2-(4-iodobutyl)isoindoline 1,3-dione (33 mg) and Py S9 (74 mg) Purification using flash silica gel column chromatography (eluent: 15:1 hexane:EtOAc) gave the pure pokuct as a colorless oil (21.9 mg, 73\% yield).

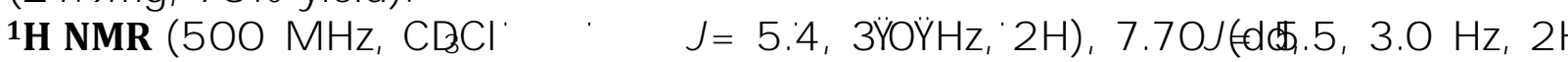
3.68 z $3.65(\mathrm{~m}, 2 \mathrm{H}), 1.67 \mathrm{z} 1.51(\mathrm{~m}, 9 \mathrm{H}), 1.47 \mathrm{z} 1.42(\mathrm{~m}, 2 \mathrm{H}), 1.41 \mathrm{z} 1.27(\mathrm{~m}, 2 \mathrm{H}), 1.34 \mathrm{z}$

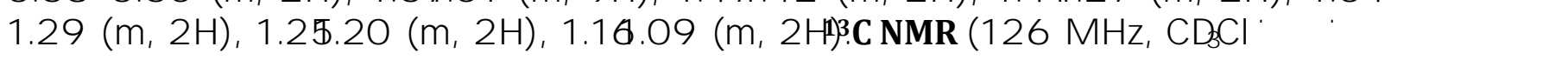
$133.8,132.2,123.1,39.1,38.1,37.7,34.5,28.9,52,26.4,24.7 \mathrm{ppm}$.

HRMS (EI): Calcd. 299.1880 for $\mathrm{C}_{9} \mathrm{H}_{25} \mathrm{NO}_{2}+[\mathrm{M}]+$, found 299.1871 .

\section{(4-(4-Bromophenoxy)butyl)cycloheptane (4ak):}<smiles>Brc1ccc(OCCCCC2CCCCCC2)cc1</smiles>

Following the GP3, the title compound was prepared from1-bromo-4-(4-iodobutoxy) benzene (35.5 mg) and Py S9 (74 mg), Purification using flash silica gel column chromatography (eluent: 50:1 hexane:EtOAc) gave the pure product as a colorless oil (23.4 mg, $72 \%$ yield).

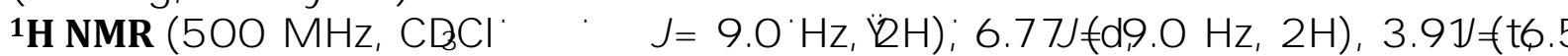
$\mathrm{Hz}, 2 \mathrm{H}), 1.77 \mathrm{z} 1.71(\mathrm{~m}, 2 \mathrm{H}), 1.70 \mathrm{z} 1.61(\mathrm{~m}, 6 \mathrm{H}), 1.51 \mathrm{z} 1.37(\mathrm{~m}, 7 \mathrm{H}), 1.29 \mathrm{z} 1.24(\mathrm{~m}, 2 \mathrm{H})$,

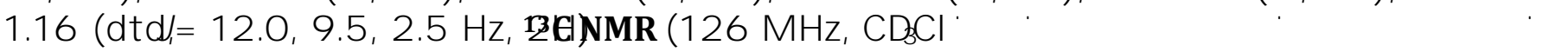
68.3, 39.2, 37.9, 34.6, 29.5, 28.36.5, 23.8 ppm.

HRMS (EI): Calcd. 324.1083 for $\mathrm{GH}_{25} \mathrm{BrO}^{+}[\mathrm{M}]^{+}$, found 324.1075 .

\section{3-Cycloheptylpropyl benzoylprolinate (4al):}<smiles>O=C(OCCCC1CCCCCC1)C1CCCN1C(=O)O</smiles>

Following the GP3, the title compound was prepared from3-iodopropyl benzoylprolinate (38.7 mg) and Py S9 (74 mg)Purification using flash silica gel column chromatography (eluent: 5:1 hexane:EtOAc) gave the pure product as a colorless ơA(7 mg, 69\% yield). 


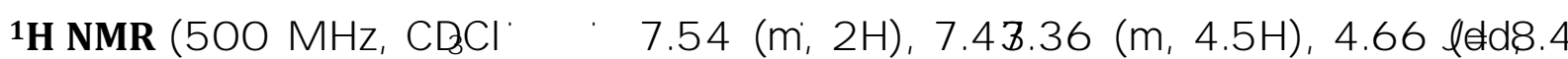
$5.1 \mathrm{~Hz}, 2 \mathrm{H}), 4.32(\mathrm{dJ}=6.4 \mathrm{~Hz}, 0.3 \mathrm{H}), 4.1 \& 4.09(\mathrm{~m}, 2 \mathrm{H}), 3.98 \mathrm{z} 3.93(\mathrm{~m}, 0.3 \mathrm{H}), 3.91 \mathrm{z}$ $3.86(\mathrm{~m}, 0.3 \mathrm{H}), 3.79(\mathrm{tJ}=6.9 \mathrm{~Hz}, 0.6 \mathrm{H}), 3.63(\mathrm{df},=10.5,7.0 \mathrm{~Hz}, 1 \mathrm{H}), 3.543 .49(\mathrm{~m}, 1 \mathrm{H})$, $2.35 \mathrm{z} 2.28(\mathrm{~m}, 1 \mathrm{H}), 2.24 \mathrm{z} 2.17(\mathrm{~m}, 0.3 \mathrm{H}), 2.05 \mathrm{z} 1.97(\mathrm{~m}, 3 \mathrm{H}), 1.90 \mathrm{z} 1.82(\mathrm{~m}, 1.3 \mathrm{H}), 1.69$ z $1.52(\mathrm{~m}, 10 \mathrm{H}), 1.48 \mathrm{z} 1.35(\mathrm{~m}, 8 \mathrm{H}), 1.29 \mathrm{z} 1.25(\mathrm{~m}, 3 \mathrm{H}), 1.18 \mathrm{z} 1.12(\mathrm{~m}, 3 \mathrm{H}) .{ }^{13} \mathrm{C}$ NMR

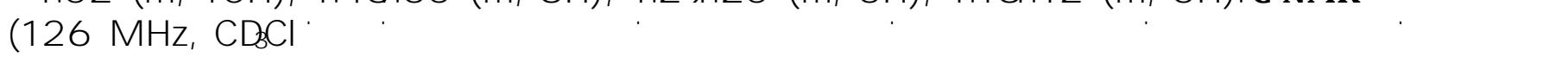
127.2 , 126.5, 65.7, 65.6, 61.5, 59.2 , 4946.5. 38.8, 38.8, 34.4, 34.4, 34.1, 33.9, 31.5, 29.4, $28.4,26.5,26.4,26.3,25.3,22.6 \mathrm{ppm}$.

HRMS (EI): Calcd. 357.2298 for $\mathrm{C}_{2} \mathrm{H}_{31} \mathrm{NO}_{3}+[\mathrm{M}]+$, found 357.2297.

\section{3-Cycloheptylpropyl 2-(11-oxo-6,11-dihydrodibenzo[b,e]oxepin-2-yl)acetate (4am):}<smiles>O=C(Cc1ccc2c(c1)C(=O)c1ccccc1CO2)OCCCC1CCCCC1</smiles>

Following the GP3, the title compound was prepared from3-iodopropyl 2-(11-oxo-6,11dihydrodibenzo[b,e]oxepin-2-yl)acetate (43.6 mg) and Py S9 (74 mg),Purification using flash silica gel column chromatography (eluent: 15:1hexane:EtOAc) gave the pure product as a colorless oil $20.7 \mathrm{mg}, 51 \%$ yield).

1H NMR (500 MHz, CDGQ I ЏJ8=2. BHHzj 1̈̈̈) $\mathrm{J}=7.5,1.3 \mathrm{~Hz}, 1 \mathrm{H}), 7.47(\mathrm{tg},=7.6,1.2 \mathrm{~Hz}, 1 \mathrm{H}), 7.43(\mathrm{dd}=8.4,2.4 \mathrm{~Hz}, 1 \mathrm{H}) 7.36(\mathrm{dd}, \mathrm{J}=$ 7.0, $0.5 \mathrm{~Hz}, 1 \mathrm{H}), 7.02(\mathrm{~g},=8.4 \mathrm{~Hz}, 1 \mathrm{H}), 5.18(\mathrm{~s}, 2 \mathrm{H}), 4.07 \mathrm{gt}=6.8 \mathrm{~Hz}, 2 \mathrm{H}), 3.63(\mathrm{~s}, 2 \mathrm{H})$, 1.63 z $1.53(\mathrm{~m}, 7 \mathrm{H}), 1.46 \mathrm{z} 1.36(\mathrm{~m}, 6 \mathrm{H}), 1.21(\mathrm{dddJ}=12.1,8.9,6.2 \mathrm{~Hz}, 2 \mathrm{H}), 1.13(\mathrm{ddj}=$

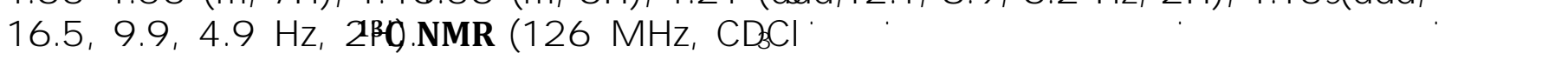
$135.5,132.7,132.4,129.5,129.2$, 128.0, 127.8, 125.1, 121.0, 73.6, 65.4, 40.3, 38.8, 34.4, $34.1,28.4,26.4,26.4 \mathrm{ppm}$.

HRMS (EI): Calcd. 406.2144 for ${ }_{66} \mathrm{H}_{30} \mathrm{O}_{4}+[\mathrm{M}]+$, found 406.2136 .

\section{3-Cycloheptylpropyl 2-(1-(4-chlorobenzoyl)-5-methoxy-2-methyl-1H-indol-3-yl) acetate (4an):}<smiles>COc1ccc2c(CC(=O)OCCCC3CCCCCC3)c(C)n(C(=O)c3ccc(Cl)cc3)c2c1</smiles>

Following the GP3, the title compound was prepared from3-iodopropyl 2-(1-(4chlorobenzoyl)-5-methoxy-2-methyl-1H-indol-3-yl)acetate (52.6 mg) and Py S9 (74mg), Purification using flash silica gel column chromatography (eluent: 15:1 hexane:EtOAc) gave the pure product as a colorless oiD5.8 $\mathrm{mg}, 52 \%$ yield).

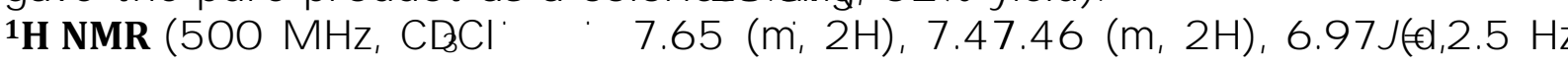
$1 \mathrm{H}), 6.87(\mathrm{~d}, \mathrm{~J}=9.0 \mathrm{~Hz}, 1 \mathrm{H}), 6.66(\mathrm{dd},=9.0,2.5 \mathrm{~Hz}, 1 \mathrm{H}), 4.07(\mathrm{f},=6.7 \mathrm{~Hz}, 2 \mathrm{H}), 3.83(\mathrm{~s}$, $3 \mathrm{H}), 3.65(\mathrm{~s}, 2 \mathrm{H}), 2.39(\mathrm{~s}, 3 \mathrm{H}), 1.631 .52(\mathrm{~m}, 9 \mathrm{H}), 1.49 \mathrm{Z} 1.41(\mathrm{~m}, 2 \mathrm{H}), 1.401 .34(\mathrm{~m}, 2 \mathrm{H})$,

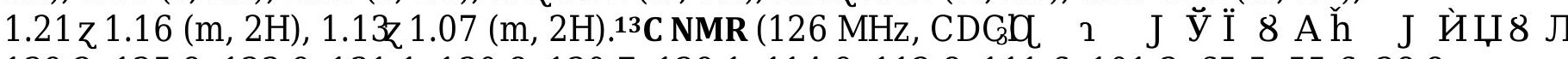
139.2 , 135.8, 133.9, 131.1, 130.8, 130.7, 129.1, 114.9, 112.8, 111.6, 101.3, 65.5, 55.6, 38.8, $34.4,34.1,30.4,28.4,26.5,26.4,13.3 \mathrm{ppm}$.

HRMS (ESI): Calcd. 518.2069 for $6 \mathrm{H}_{34} \mathrm{ClNNaQ}^{+}(\mathrm{M}+\mathrm{Na})^{+}$, found 518.2060. 
2-(6-(2,6-Dimethylphenoxy)-5-methylhexyl)isoindoline-1,3-dione (4ao):<smiles>Cc1cccc(C)c1OCC(C)CCCCN1C(=O)c2ccccc2C1=O</smiles>

Following the GP3, the title compound was prepared from2-(4-iodobutyl)isoindoline 1,3-dione (33 mg) and Py S11 (84 mg) Purification using flash silica gel column chromatography (eluent: 15:1 hexane:EtOAc) gave the pure product as a colorless oil (12.1 mg, 33\% yield).

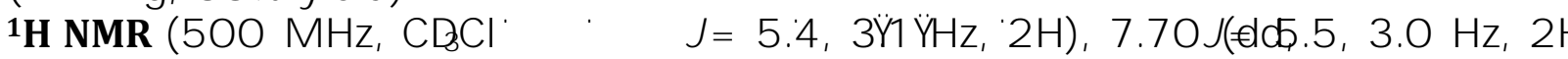
$6.98(\mathrm{~d}, \mathrm{~J}=7.5 \mathrm{~Hz}, 2 \mathrm{H}), 6.9 \mathrm{Q} 6.87(\mathrm{~m}, 1 \mathrm{H}), 3.71(\mathrm{tJ}=7.3 \mathrm{~Hz}, 2 \mathrm{H}), 3.58(\mathrm{dd},=8.8,5.8 \mathrm{~Hz}$, $1 \mathrm{H}), 3.52(\mathrm{dd}, \mathrm{J}=8.8,6.4 \mathrm{~Hz}, 1 \mathrm{H}), 2.24(\mathrm{~s}, 6 \mathrm{H}), 1.94(\mathrm{fd}=12.9,6.4 \mathrm{~Hz}, 1 \mathrm{H}), 1.781 .69$ $(\mathrm{m}, 1 \mathrm{H}), 1.67 \mathrm{z} 1.61(\mathrm{~m}, 1 \mathrm{H}), 1.54 \mathrm{z} 1.46(\mathrm{~m}, 1 \mathrm{H}), 1.43 \mathrm{z} 1.37(\mathrm{~m}, 1 \mathrm{H}), 1.34 \mathrm{z} 1.27(\mathrm{~m}, 1 \mathrm{H})$,

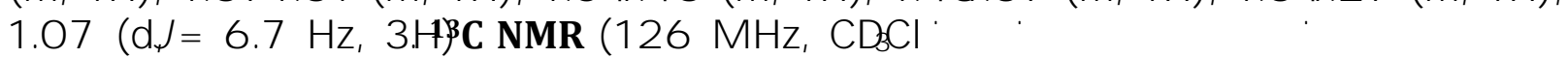
$128.8,123.6,123.1,77.0,38.0,34.2,33.0,28.9,24.4,17.0,16.2 \mathrm{ppm}$.

HRMS (EI): Calcd. 365.1985 for ${ }_{83} \mathrm{H}_{27} \mathrm{NO}_{3}+[\mathrm{M}]+$, found 365.1982 .

\section{2-(5-Methyldecyl)isoindoline-1,3-dione (4ap):}

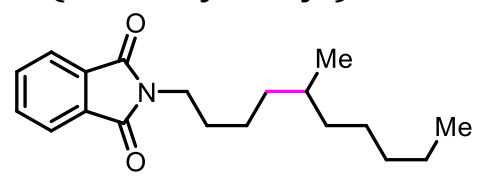

Following the GP3, the title compound was prepared from2-(4-iodobutyl)isoindoline 1,3-dione (33 mg) and Py S10 (74 mg), Purification using flash silica gel column chromatography (eluent: 15:1 hexane:EtOAc) gave the purerquduct as a colorless oil (18.7 mg, 62\% yield).

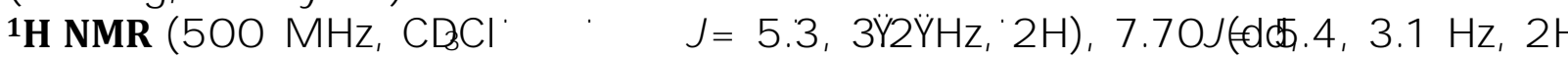
$3.67(\mathrm{t}, \mathrm{J}=7.4 \mathrm{~Hz}, 2 \mathrm{H}), 1.6 \mathrm{Z} 1.61(\mathrm{~m}, 2 \mathrm{H}), 1.37 \mathrm{z} 1.21(\mathrm{~m}, 11 \mathrm{H}), 1.15 \mathrm{z} 1.04(\mathrm{~m}, 2 \mathrm{H}), 0.86$ $(\mathrm{t}, \mathrm{J}=7.1 \mathrm{~Hz}, 3 \mathrm{H}), 0.82(\mathrm{~d},=6.4 \mathrm{~Hz}, 3 \mathrm{H}) \mathbf{1}^{\mathbf{3}} \mathbf{C} \mathbf{N M R}(126 \mathrm{MHz}, \mathrm{CDG} \mathrm{Q} \quad \mathrm{J}$ Й $123.1,38.1,36.9,36.5,32.6,32.2,28.9,26.7,24.3,22.7,19.6,14.1 \mathrm{ppm}$.

HRMS (EI): Calcd. 301.2036 for $\mathrm{C}_{9} \mathrm{H}_{27} \mathrm{NO}_{2}+[\mathrm{M}]+$, found 301.2040.

2-(5,6-Dimethylheptyl)isoindoline-1,3-dione (4aq):<smiles>CC(C)C(C)CCCN1C(=O)c2ccccc2C1=O</smiles>

Following the GP3, the title compound was prepared from2-(4-iodobutyl)isoindoline 1,3-dione (33 mg) and Py S5 (70 mg,) Purification using flash silica gel column chromatography (eluent: 15:1 hexane:EtOAc) gave the pure product as a colorless oil (13.7 $\mathrm{mg}, 50 \%$ yield).

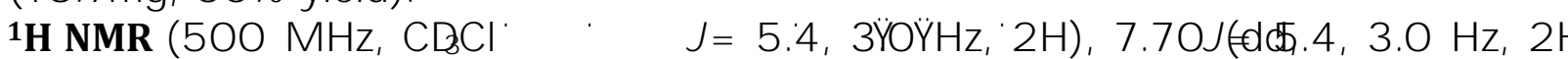
$3.67(\mathrm{t}, \mathrm{J}=7.4 \mathrm{~Hz}, 2 \mathrm{H}), 1.621 .63(\mathrm{~m}, 2 \mathrm{H}), 1.56 \mathrm{z} 1.48(\mathrm{~m}, 1 \mathrm{H}), 1.37 \mathrm{z} 1.24(\mathrm{~m}, 5 \mathrm{H}), 1.15$ z $1.07(\mathrm{~m}, 1 \mathrm{H}), 0.83(\mathrm{dJ}=6.8 \mathrm{~Hz}, 3 \mathrm{H}), 0.78(\mathrm{~d} J,=4.3 \mathrm{~Hz}, 3 \mathrm{H}), 0.77(\mathrm{~d},=4.2 \mathrm{~Hz}, 3 \mathrm{H}){ }^{13} \mathrm{C}$

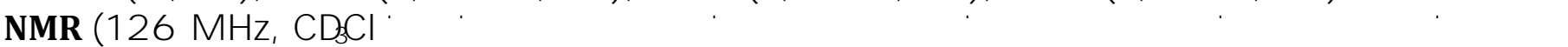
18.0, $15.3 \mathrm{ppm}$.

HRMS (EI): Calcd. 273.1723 for $\mathrm{GH}_{23} \mathrm{NO}_{2}+[\mathrm{M}]^{+}$, found 273.1724 .

(6-(Benzyloxy)-3-methylhexyl)benzene (4ar): 
<smiles>CC(CCCOCc1ccccc1)CCc1ccccc1</smiles>

Following the GP3, the title compound was prepared from((3-iodopropoxy)methyl) benzene (27.6 mg) and Py S3 (79 mg) Purification using flash silica gel column chromatography (eluent: 40:1 hexane:EtOAc) gave the pure product as a colorless oil (20.1 mg, 71\% yield).

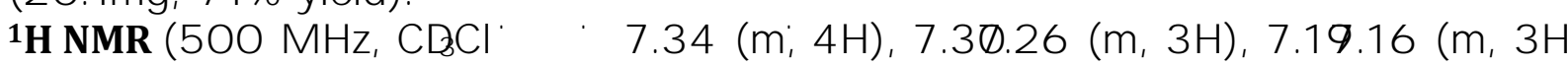
$4.51(\mathrm{~s}, 2 \mathrm{H}), 346(\mathrm{t}, \mathrm{J}=6.7 \mathrm{~Hz}, 2 \mathrm{H}), 2.65(\mathrm{ddd},=15.4,10.5,5.1 \mathrm{~Hz}, 1 \mathrm{H}), 2.57(\mathrm{dd} \bar{d}=$ 13.7, 9.8, $6.1 \mathrm{~Hz}, 1 \mathrm{H}), 1.7 \mathrm{\ell} 1.60(\mathrm{~m}, 3 \mathrm{H}), 1.51 \mathrm{z} 1.40(\mathrm{~m}, 3 \mathrm{H}), 1.29 \mathrm{z} 1.20(\mathrm{~m}, 1 \mathrm{H}), 0.95$

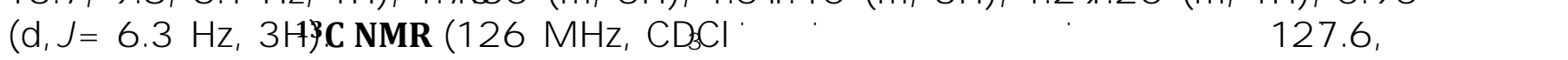
$127.5,127.2,127.1,125.5,72.9,70.8,38.8,33.4,33.2,32.3,27.2,19.5$ ppm.

HRMS (EI): Calcd. 282.1978 for ${ }_{60} \mathrm{H}_{26} \mathrm{O}^{+}[\mathrm{M}]+$, found 282.1986.

\section{7-((5-Methyl-7-phenylheptyl)oxy)-2H-chromen-2-one (4as):}<smiles>CC(CCCCOc1ccc2ccc(=O)oc2c1)CCc1ccccc1</smiles>

Following the GP3, the title compound was prepared from methyl 4bromobe7-(4iodobutoxy)-2H-chromen-2-onenzoate (34.4 mg) and Py S3 (79 mg),Purification using flash silica gel column chromatography (eluent: 5:1 hexane:EtOAc) gave the pure product as a colorless oil $\$ 2.1 \mathrm{mg}, 63 \%$ yield).

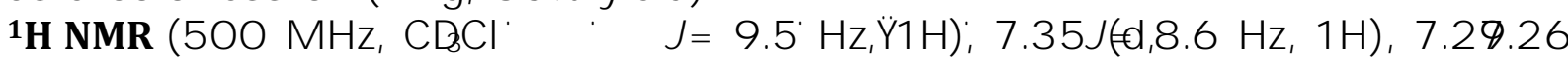
$(\mathrm{m}, 2 \mathrm{H}), 7.19 \mathrm{z} 7.16(\mathrm{~m}, 3 \mathrm{H}), 6.83(\mathrm{ddJ}=8.6,2.4 \mathrm{~Hz}, 1 \mathrm{H}), 6.80(\mathrm{~d},=2.3 \mathrm{~Hz}, 1 \mathrm{H}), 6.24(\mathrm{~d}$, $\mathrm{J}=9.5 \mathrm{~Hz}, 1 \mathrm{H}), 4.01(\mathrm{~g}=6.5 \mathrm{~Hz}, 2 \mathrm{H}), 2.66(\mathrm{ddd},=13.6,10.5,5.2 \mathrm{~Hz}, 1 \mathrm{H}), 2.57(\mathrm{dd} d=$ 13.7, 10.0, $6.1 \mathrm{~Hz}, 1 \mathrm{H}), 1.8 \mathrm{Z} 1.76(\mathrm{~m}, 2 \mathrm{H}), 1.68 \mathrm{z} 1.62(\mathrm{~m}, 1 \mathrm{H}), 1.52 \mathrm{z} 1.40(\mathrm{~m}, 5 \mathrm{H}), 1.28$

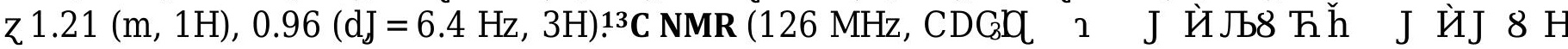
143.4, 142.9, 128.7, 128.3, 128.2, 125.56, 125.56, 113.0, 112.9, 112.3, 101.3, 68.6, 38.8, $36.5,33.4,32.4,29.2,23.3,19.5 \mathrm{ppm}$.

HRMS (EI): Calcd. 350.1876 for $\mathrm{E}_{3} \mathrm{H}_{26} \mathrm{O}^{+}[\mathrm{M}]^{+}$, found 350.1873 .

\section{6-Methyl-8-phenyloctanal (4at):}<smiles>CC(CCCCC=O)CCc1ccccc1</smiles>

Following the GP3, the title compound was prepared from 5iodopentanal (21 $\mathrm{mg}$ ) and Py S3 (79 mg) using 20 mol\% Ni and 25 mol\% L2, Purification using flash silica gel column chromatography (eluent: 15:1 hexane:EtOAc) gave the pure product as a colorløis( 9.8 $\mathrm{mg}, 45 \%$ yield).

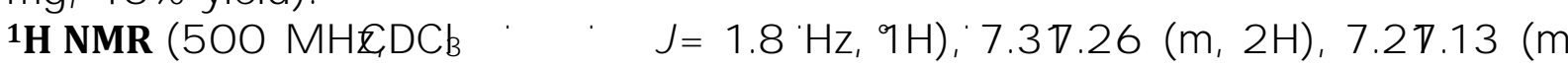
$3 \mathrm{H}), 2.65(\mathrm{ddd}, \mathrm{J}=13.5,10.5,5.2 \mathrm{~Hz}, 1 \mathrm{H}), 2.55(\mathrm{ddd}=13.6,9.9,6.1 \mathrm{~Hz}, 1 \mathrm{H}), 2.42(\mathrm{tg}=$ 7.4, $1.9 \mathrm{~Hz}, 1 \mathrm{H}), 1.6$ қ $1.55(\mathrm{~m}, 4 \mathrm{H}), 1.44(\mathrm{dttJ}=9.8,5.7,2.0 \mathrm{~Hz}, 2 \mathrm{H}), 1.3801 .28(\mathrm{~m}, 3 \mathrm{H})$,

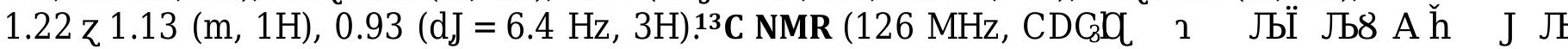
128.3, 125.6, 43.9, 38.8, 36.6, 33.4, 32.3, 26.5, 22.4, 1pqpm.

HRMS (ESI) for: $\mathrm{G}_{5} \mathrm{H}_{22} \mathrm{ONa}^{+}[\mathrm{M}+\mathrm{Na}]^{+}$: calcd 241.1563, found 241.1566. 
Methyl 4-(1-(2-(11-oxo-6,11-dihydrodibenzo[b,e]oxepin-2-yl)acetoxy)butan-2yl)benzoate (4au):<smiles>CCC(COC(=O)Cc1ccc2c(c1)C(=O)c1ccccc1CO2)c1ccc(C(C)=O)cc1</smiles>

Following the GP2, the title compound was prepared frommethyl 4-bromobenzoate (21.5 mg) and Py S12 (108 mg, 1.5 equiv) Purification using flash silica gel column chromatography (eluent: 15:1 hexane:EtOAc) gave the pure product as a colorless oil (26.5 mg, 58\% yield).

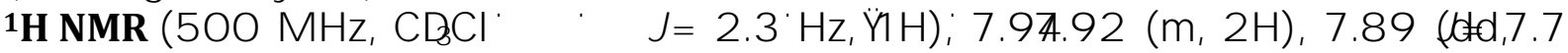
$1.1 \mathrm{~Hz}, 1 \mathrm{H}), 7.56(\mathrm{tdJ}=7.5,1.3 \mathrm{~Hz}, 1 \mathrm{H}), 7.47(\mathrm{tg},=7.6,1.2 \mathrm{~Hz}, 1 \mathrm{H}), 7.36(\mathrm{~g},=7.4 \mathrm{~Hz}$, $1 \mathrm{H}), 7.25(\mathrm{dd}, \mathrm{J}=8.4,2.4 \mathrm{~Hz}, 1 \mathrm{H}), 7.19(\mathrm{~d},=8.3 \mathrm{~Hz}, 2 \mathrm{H}), 6.95(\mathrm{~d},=8.4 \mathrm{~Hz}, 1 \mathrm{H}), 5.18(\mathrm{~s}$, 2H), $4.26 \mathrm{z} 4.25$ (m, 2H), $3.88(\mathrm{~s}, 3 \mathrm{H}), 3.54(\mathrm{~s}, 2 \mathrm{H}), 2.89$ (dgd $12.5,9.3,6.8 \mathrm{~Hz}, 1 \mathrm{H}), 1.79$ z $1.71(\mathrm{~m}, 1 \mathrm{H}), 1.63 z 1.54(\mathrm{~m}, 1 \mathrm{H}), 0.79(\mathrm{tJ}=7.4 \mathrm{~Hz}, 3 \mathrm{H}){ }^{\mathbf{1}} \mathbf{C}$ NMR $(126 \mathrm{MHz}, \mathrm{CDG} \mathrm{I}$ 190.7, 171.1, 166.9, 160.4, 147.1, 140.4, 136.2, 135.6, 132.7, 13224,.7, 129.5, 129.2, 128.6, 127.9, 127.8, 127.6, 125.0, 121.0, 73.6, 68.1, 52.0, 46.6, 40.2, 25.2, 11.7 ppm.

HRMS (ESI): Calcd. 481.1622 for $_{8} \mathrm{H}_{26} \mathrm{NaO}_{6}{ }^{+}(\mathrm{M}+\mathrm{Na})^{+}$, found 481.1618 .

\subsection{Site-selective alkylation by orthogonal halide activation}

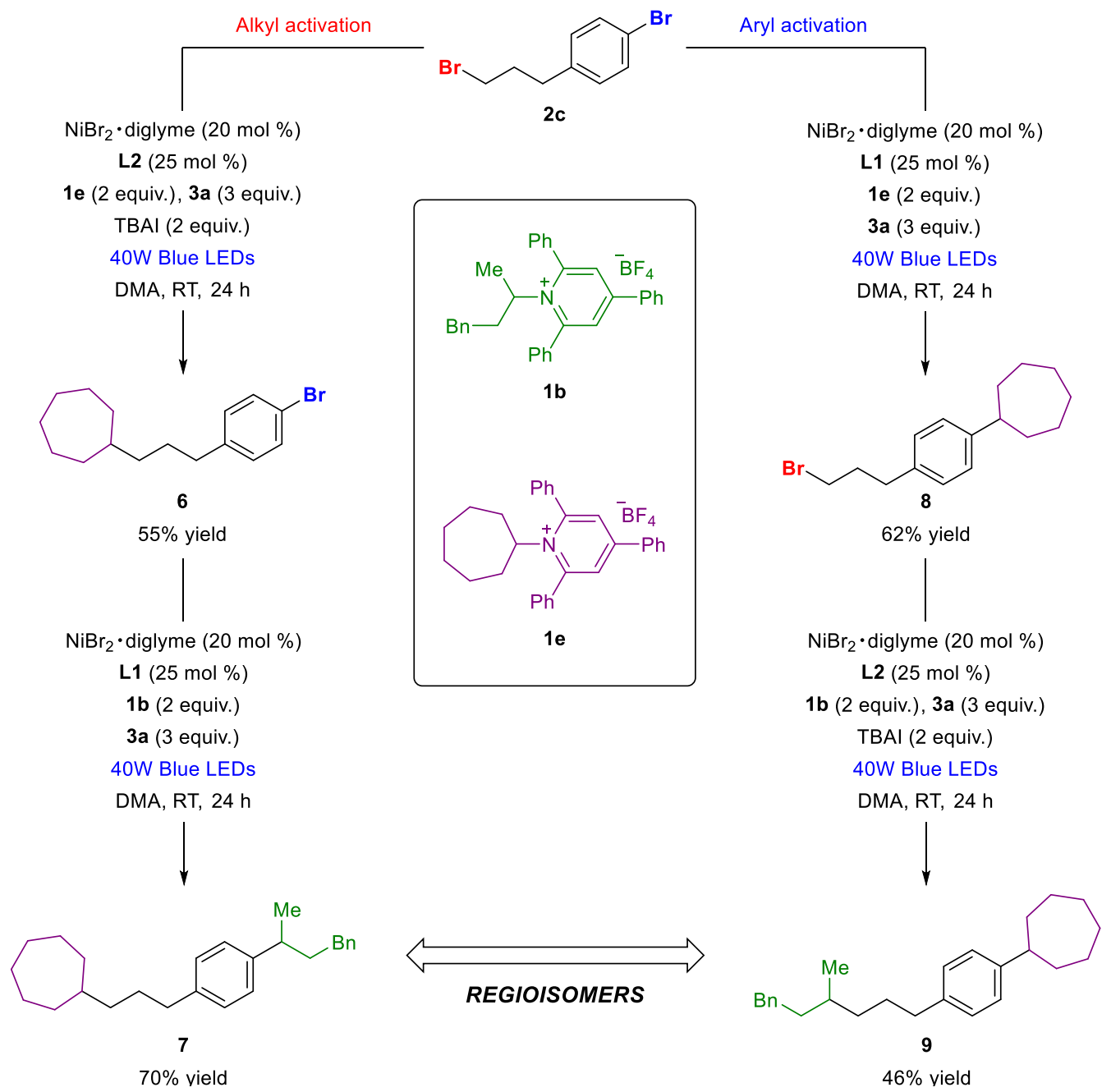




\section{(3-(4-Bromophenyl)propyl)cycloheptane (6):}<smiles>Brc1ccc(CCCC2CCCCC2)cc1</smiles>

Following the GP3, the title compound was prepared from1-bromo-4-(3-bromopropyl) benzene ( $28 \mathrm{mg}$ ) and 1e (98 $\mathrm{mg}, 2.0$ equiv.)using $20 \mathrm{~mol} \% \mathrm{NiBr} \cdot$ diglyme, $25 \mathrm{~mol} \% \mathbf{L 2}$, 3.0 equiv. 3a and 2.0 equiv. TBAhs addtitve, Purification using flash silica gel column chromatography (eluent: 50:1 hexane:EtOAc) gave the pure product as a colorless oil (16.2 mg, 55\% yield).

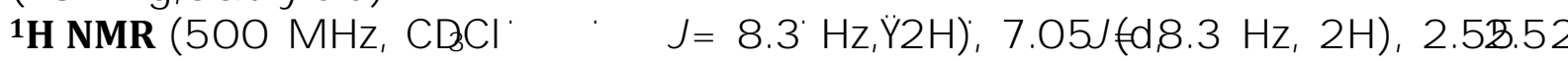
$(\mathrm{m}, 2 \mathrm{H}), 1.69 \mathrm{z} 1.54(\mathrm{~m}, 8 \mathrm{H}), 1.50 \mathrm{z} 1.36(\mathrm{~m}, 5 \mathrm{H}), 1.25 \mathrm{z} 1.21(\mathrm{~m}, 2 \mathrm{H}), 1.18 \mathrm{z} 1.11(\mathrm{~m}, 2 \mathrm{H})$. ${ }^{13}$ C NMR $(126$ MHz, CDGQ 1 J hJ 8 A h J Ы 8 Љh J ЊÏ 8 J h̆ J J A 8 Љh̆ $26.5 \mathrm{ppm}$.

HRMS (EI): Calcd. 294.0978 for $\mathrm{C}_{6} \mathrm{H}_{23} \mathrm{Br}^{+}[\mathrm{M}]+$, found 294.0983.

(3-(4-(4-Phenylbutan-2-yl)phenyl)propyl)cycloheptane (7):<smiles>C[C@@H](CCc1ccccc1)c1ccc(CCC2CCCCC2)cc1</smiles>

Following the GP2, the title compound was prepared from6 (29.4 mg) and $\mathbf{1 b}$ (105 mg, 2.0 equiv.) using $20 \mathrm{~mol} \% \mathrm{NiB}$ riglyme, $25 \mathrm{~mol} \% \mathbf{L 1}$ and 3.0 equiv3a, Purification using flash silica gel column chromatography (eluent:50:1 hexane:EtOAc) gave the pure product as a colorless oil $\$ 4.4 \mathrm{mg}, 70 \%$ yield).

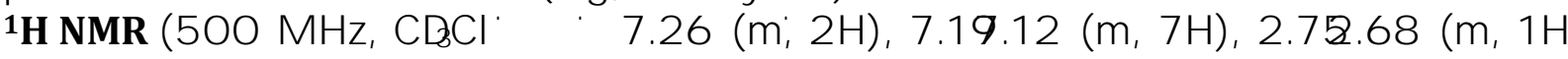
2.59 z $2.49(\mathrm{~m} 4 \mathrm{H}), 1.98 \mathrm{z} 1.85(\mathrm{~m}, 2 \mathrm{H}), 1.73 \mathrm{z} 1.69(\mathrm{~m}, 2 \mathrm{H}), 1.66 \mathrm{z} 1.57(\mathrm{~m}, 6 \mathrm{H}), 1.51 \mathrm{z}$ $1.38(\mathrm{~m}, 5 \mathrm{H}), 1.31 \mathrm{z} 1.27(\mathrm{~m}, 5 \mathrm{H}), 1.2 \mathrm{z} 1.15(\mathrm{~m}, 2 \mathrm{H}) ;{ }^{13} \mathbf{C}$ NMR $(126 \mathrm{MHz}, \mathrm{CDGQ} \quad 1$ J h h $8 \mathrm{~h} \mathrm{~h}$ 142.7, 140.5, 1284, 128.3, 128.2, 1269, 125.6, 40.0, 392, 39.1, 38.0, 35.9, 34.6, 3.0, 29.4, 28.5, 266, 22.5ppm.

HRMS (EI) for: $\mathrm{C}_{26} \mathrm{H}_{36}[\mathrm{M}]^{+}$: calcd 348.28D, found 348.2821.

(4-(3-Bromopropyl)phenyl)cycloheptane (8):<smiles>BrCCc1ccc(C2CCCCC2)cc1</smiles>

Following the GP2, the title compound was prepared from 1bromo-4-(3-bromopropyl) benzene ( $28 \mathrm{mg}$ ) andle (98 mg, 2.0 equiv.) using $20 \mathrm{~mol} \%$ NiBidiglyme, $25 \mathrm{~mol} \% \mathbf{L 1}$, and 3.0 equiv.3a, Purification using flash silica gel column chromatography (eluenÆ0:1 hexane:EtOAc) gave the pure product as a colorless oili( $2 \mathrm{mg}, 62 \%$ yield).

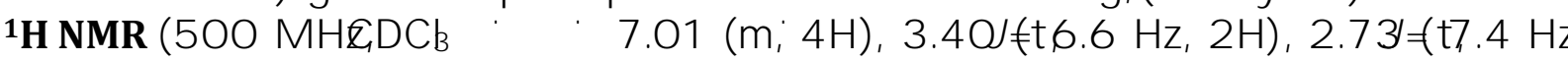
$2 \mathrm{H}), 2.64(\mathrm{tt}, \mathrm{J}=10.5,3.7 \mathrm{~Hz}, 1 \mathrm{H}), 2.16(\mathrm{dq},=8.3,6.6 \mathrm{~Hz}, 2 \mathrm{H}), 1.89(\mathrm{dddJ},=13.4,6.6$, $3.1,1.8 \mathrm{~Hz}, 2 \mathrm{H}), 1.79(\mathrm{dtd},=13.4,6.4,3.2 \mathrm{~Hz}, 2 \mathrm{H}), 1.731 .58(\mathrm{~m}, 6 \mathrm{H}), 1.53(\mathrm{ddJ}=9.7$,

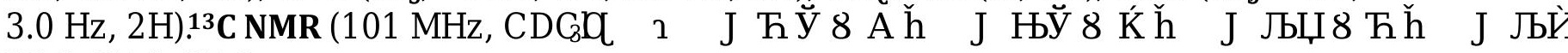
33.3, 27.9, 27.2 .

HRMS (EI) for: $\mathrm{C}_{16} \mathrm{H}_{23} \mathrm{Br}^{+}[\mathrm{M}]+$ : calcd 294.0978, found 294.0978.

(4-(4-Methyl-6-phenylhexyl)phenyl)cycloheptane (9): 
<smiles>CC(CCCc1ccc(C2CCCCC2)cc1)CCc1ccccc1</smiles>

Following the GP3, the title compound was prepared from6 (29.4 mg) and1 $\mathbf{b}$ ( $98 \mathrm{mg}, 2.0$ equiv.) using $20 \mathrm{~mol} \% \mathrm{NiBz}$ diglyme, $25 \mathrm{~mol} \% \mathbf{L 2}, 3.0$ equiv.3a and 2.0 equiv. TBAI as additive, Purification using flash silica gel column chromatography (eluent:50:1 hexane:EtOAc) gave the pure product as a colorless olib( $\mathrm{mg}, 46 \%$ yield).

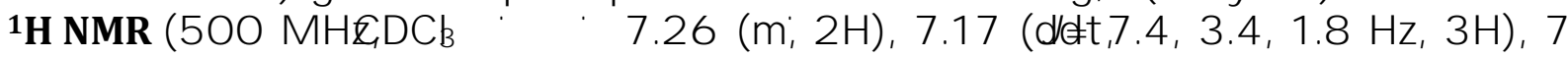
$7.06(\mathrm{~m}, 4 \mathrm{H}), 2.64(\mathrm{ddt} J=10.5,6.9,4.5 \mathrm{~Hz}, 2 \mathrm{H}), 2.5 \mathrm{~g} 2.50(\mathrm{~m}, 3 \mathrm{H}), 1.93 z 1.87(\mathrm{~m}, 2 \mathrm{H})$, $1.78(\mathrm{dp}, \mathrm{J}=10.0,3.3 \mathrm{~Hz}, 2 \mathrm{H}), 1.7 \mathrm{z} 1.59(\mathrm{~m}, 9 \mathrm{H}), 1.55 \mathrm{Z} 1.45(\mathrm{~m}, 3 \mathrm{H}), 1.461 .35(\mathrm{~m}, 2 \mathrm{H})$, 1.23 (tdd, J = 10.9, 6.1, $2.1 \mathrm{~Hz}, 1 \mathrm{H}), 0.93(\mathrm{~d}=6.4 \mathrm{~Hz}, 3 \mathrm{H}){ }^{3}{ }^{13} \mathbf{~ N M R}\left(101 \mathrm{MHz}, \mathrm{CDG}_{3} \mathrm{Q} \quad 1\right.$ $147.3,143.1,139.9,128.3,128.2,128.2,126.5,126.5,125.5,46.6,38.8,36.9,36.6,35.8$, $33.4,32.4,28.9,28.0,27.2,19$ ppom.

HRMS (EI) for: $\mathrm{C}_{26} \mathrm{H}_{36}[\mathrm{M}]^{+}$: calcd348.2812, found 348.2823.

\subsection{Attempted deaminative alkylation using metal reductants}

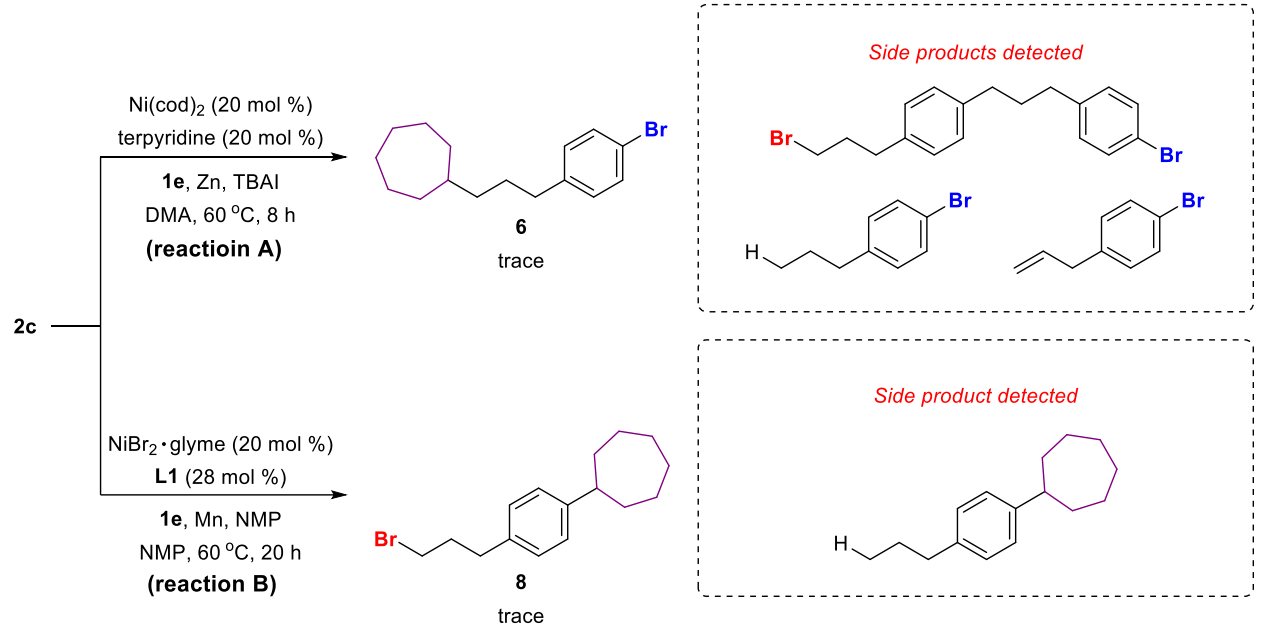

For reaction A, following the reported procedure ${ }^{10}$, after the reaction was stirred at indicated time, quenched with EtOActhe mixture was analysed by G.MS. Trace product was detected by GEMS and the side products were also analysed.

For reaction B, following the reported procedure, after the reaction was stirred at indicated time, quenched with EtOAc, the mixture wasnalysed by GeMS. Trace product was detected by GQMS and the side products were also analysed.

\subsection{Attempted cross-coupling to access 4 at using previous reductive methods}

The reactions were performed according to the reported procedures $!^{0}$ After the reaction mixture was stirred for the indicated time, the mixture was quenched with EtOAc and analyzed by GeMS. No crosscoupling product was detected and the alkyl iodide was completely consumed. 

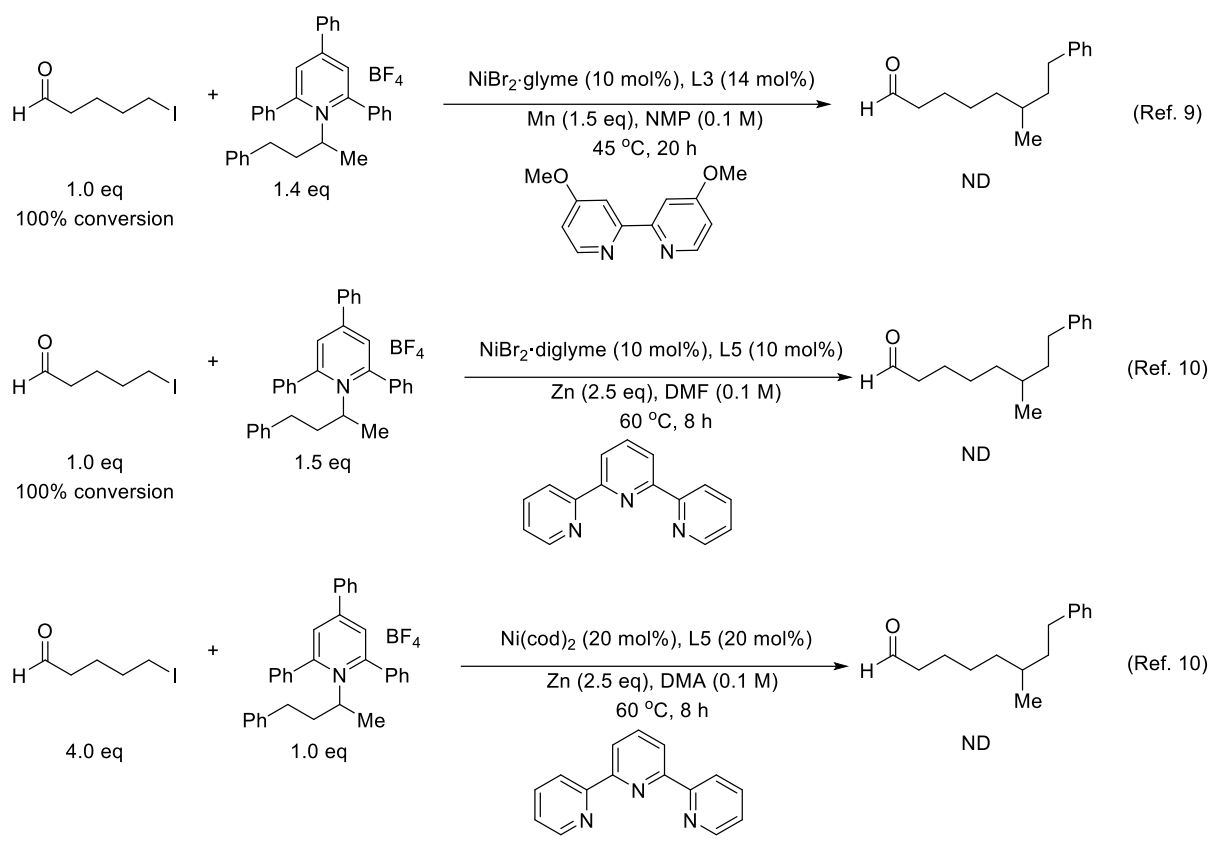

\section{Mechanistic Studies}

\subsection{Racemization experiment}

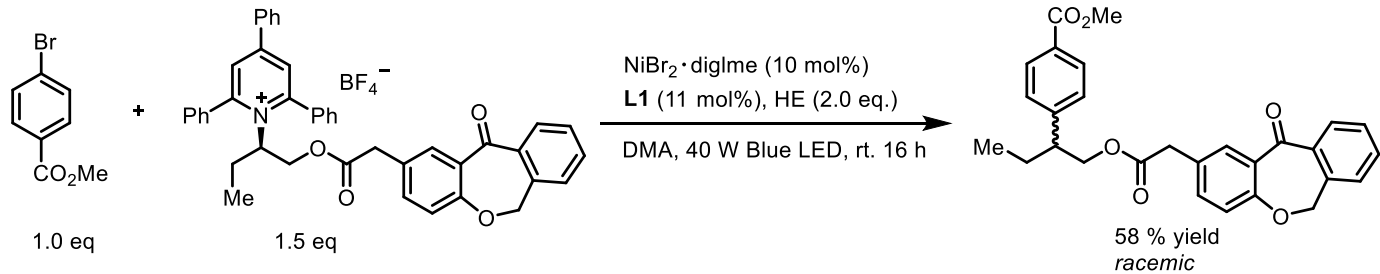

To an ovendried $5 \mathrm{~mL}$ vial equipped with a magnetic stir bar was added methyl -4 bromobenzoate $21.5 \mathrm{mg}, 0.1 \mathrm{mmol})$, pyridinium salts $(0.13 \mathrm{mmol}) 3 \mathbf{3 a}(51 \mathrm{mg}, 0.2 \mathrm{mmol})$, and 4,4 '-dimethoxy-2,2'-bipyridine $(0.011 \mathrm{mmol})$. The vial was transferred to a $\$$-filled glovebox. $\mathrm{NiBr}$ (diglyme) $(0.01 \mathrm{mmol})$ was added to the vial, and the mixture was then dissolved in $1.0 \mathrm{~mL}$ DMA. The vial was tightly sealed and removed from the glovebokeT reaction was allowed to stir under $40 \mathrm{~W}$ blue LED irradiation for $16 \mathrm{~h}$ with fans to maintain the temperature below $30^{\circ} \mathrm{C}$ (the vial was placed about $3 \mathrm{~mm}$ from the LEDs, see experimental setup)After that, the reaction was diluted with $6 \mathrm{~mL}$ EtOAc, and washed with saturated aq. $\mathrm{NaCl}(2 \times 3 \mathrm{~mL})$, the organic was separated and concentrated under reduced pressure.The residue was subjected to flash silica gel column chromatography to afford the pure product.

Enantiomeric excess was determined by HPLC (IB column, hexane/iPrOH 90/10, 0.60 $\mathrm{mL} / \mathrm{min}, 1254 \mathrm{~nm}): \mathrm{t}_{1}=34.3 \mathrm{~min}, \mathrm{t}_{2}=35.1 \mathrm{~min} .0 \%$ ee.

HPLC spectra 


\section{LabSolutions Analysis Report}

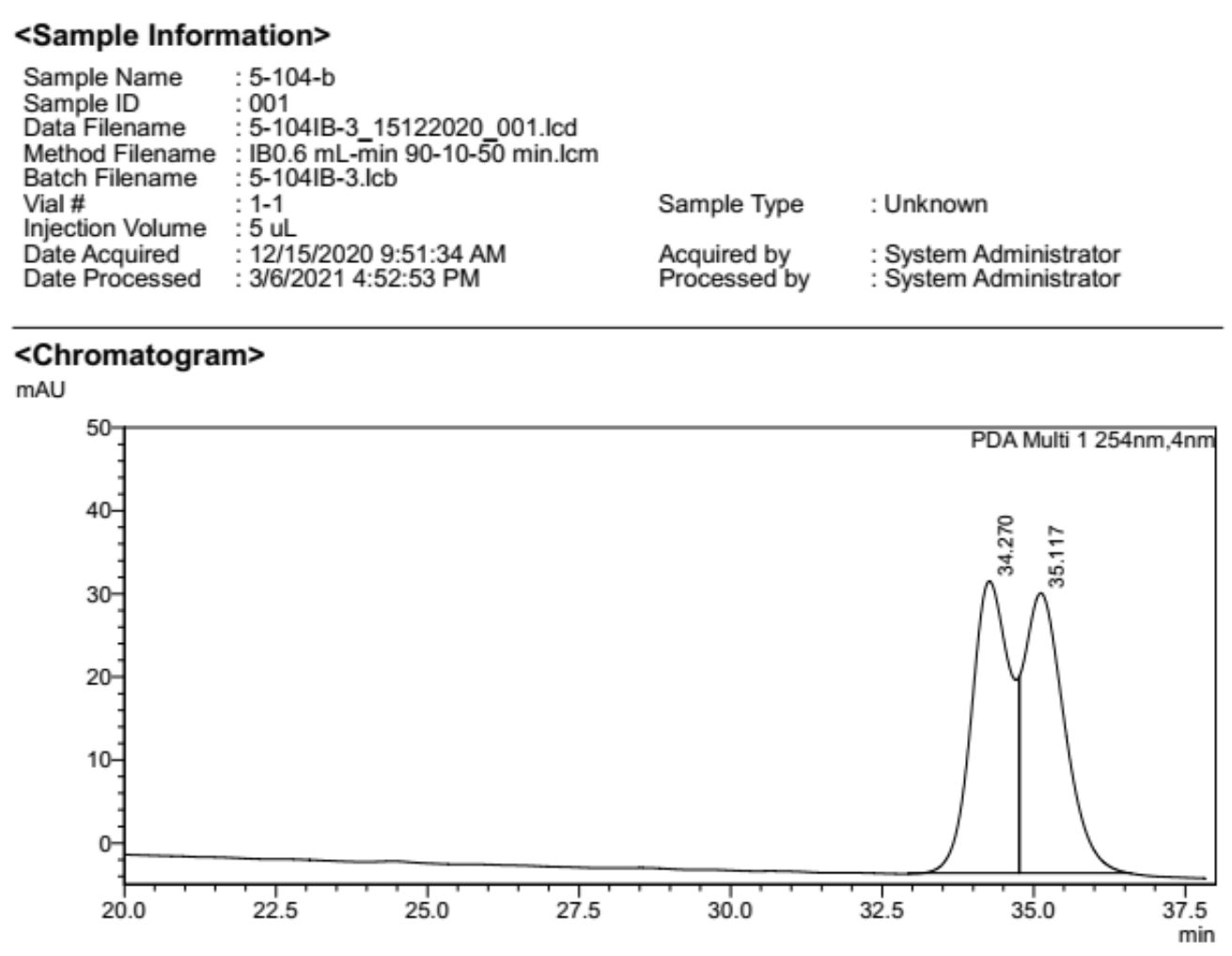

<Peak Table>

\begin{tabular}{|c|c|c|c|c|}
\hline \multicolumn{5}{|c|}{ PDA Ch1 254nm } \\
\hline Peak\# & Ret. Time & Area & Height & Area $\%$ \\
\hline 1 & 34.270 & 1564763 & 35125 & 50.168 \\
\hline 2 & 35.117 & 1554273 & 33683 & 49.832 \\
\hline Total & & 3119036 & 68808 & 100.000 \\
\hline
\end{tabular}

\subsection{Radical inhibition experiment}
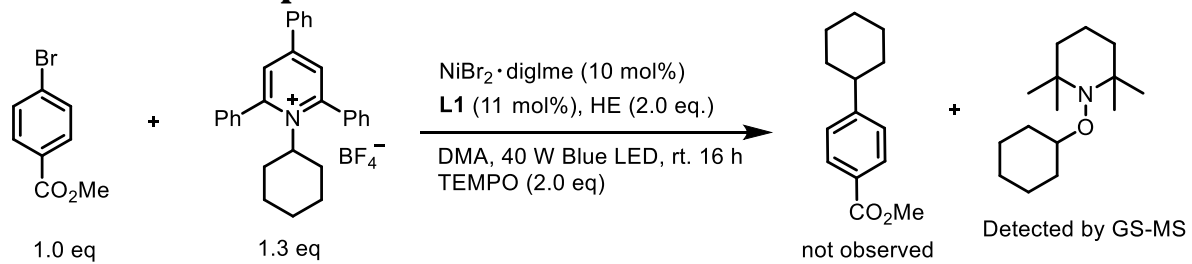

To an ovendried $5 \mathrm{~mL}$ vial equipped with a magnetic stir bar was added methyl 4 bromobenzoate $(21.5 \mathrm{mg}, 0.1 \mathrm{mmol})$, pyridinium salts $(0.13 \mathrm{mmol})$, $\mathrm{HE}$ (51 $\mathrm{mg}, 0.2$ $\mathrm{mmol}$ ), TEMPO (31 mg, $0.2 \mathrm{mmol})$, and 4; 4 imethoxy-2,2'-bipyridine $(0.011 \mathrm{mmol})$. The vial was transferred to a $\mathrm{N}$-filled glovebox. $\mathrm{NiBk}$ (diglyme) $(0.01 \mathrm{mmol})$ was added to the vial, and the mixture was then dissolved in $1.0 \mathrm{~mL}$ DMA. The vial was tightly sealed and removed from the glovebox. Te reaction was allowed to stir under $40 \mathrm{~W}$ blue LED irradiation for $16 \mathrm{~h}$ with fans to maintain the temperature below $30{ }^{\circ} \mathrm{C}$ (the vial was placed about $3 \mathrm{~mm}$ from the LEDs, see experimental setupffter that, the reaction 
mixture was analyzed by GEmass and the corresponding TEMP-Qdduct was detected by GC mass spectroscopy.
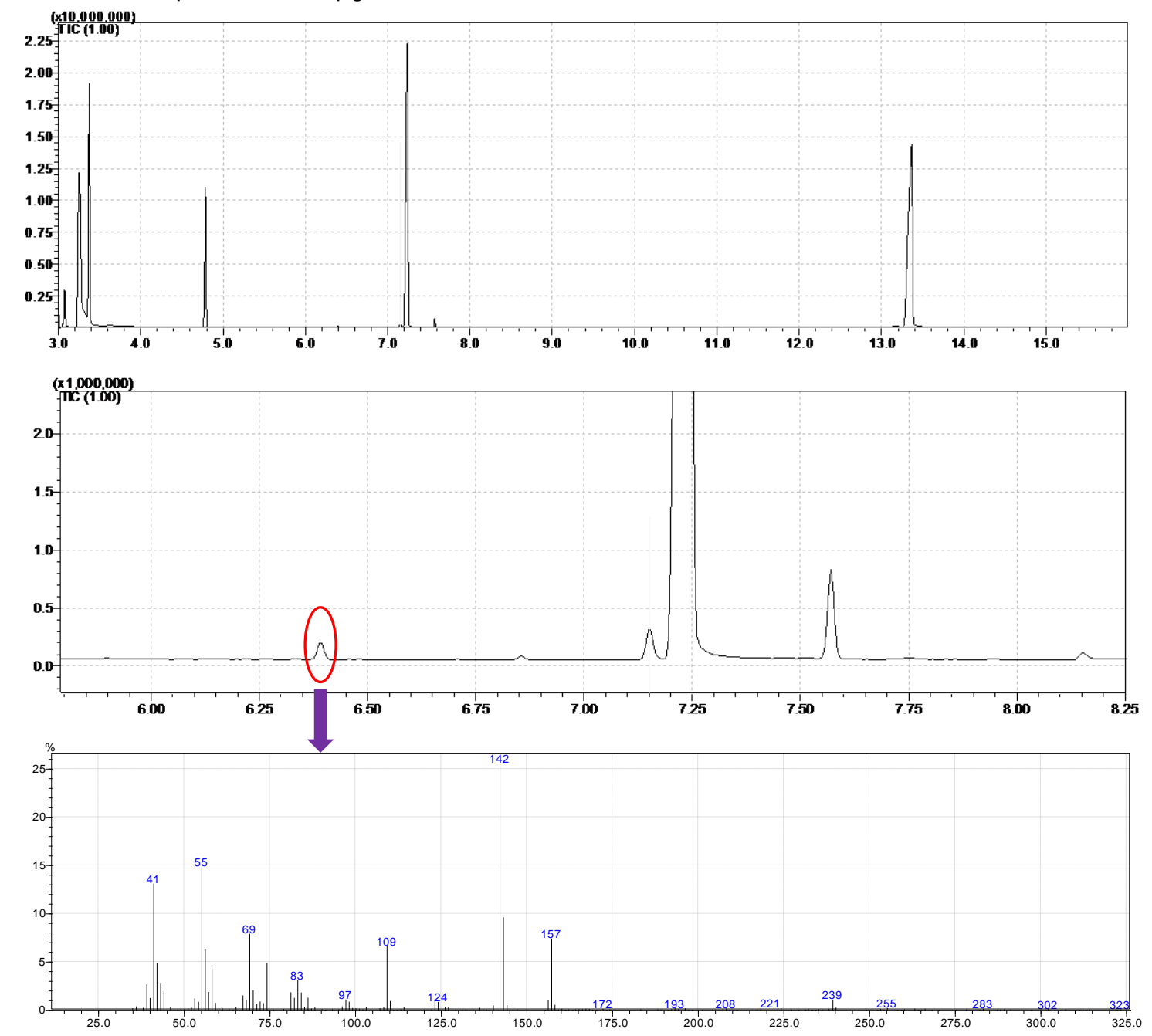

\subsection{Synthesis of oxidative addition complex $\mathrm{Ni}-1$}<smiles>[Mg]c1ccnc(-c2cc(Br)ccn2)c1</smiles>

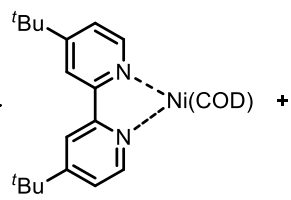<smiles>CC(=O)c1ccc(Br)cc1</smiles>

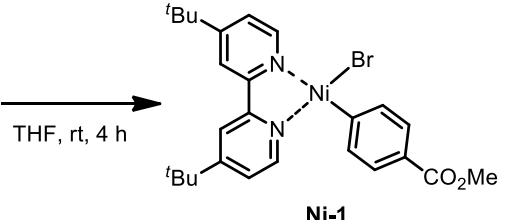

In a nitrogen filled glove box, a50 mL round bottom flask containing a stirring bar was charged with $\mathrm{Ni}(\mathrm{COD} 2$ (138 mg, $0.5 \mathrm{mmol}, 1.0$ equiv), 4,4'di-tert-butyl-2,2'-bipyridine (134 mg, $0.5 \mathrm{mmol}, 1.0$ equiv) and dry THF $\$ \mathrm{~mL}$ ) giving a dark purple mixture which was stirred for overnight at $25^{\circ} \mathrm{C}$. Methyl 4-bromobenzoate (1.1g, $5 \mathrm{mmol}, 10.0$ equiv) was added and stirred for additional4 $\mathrm{h}$. Dry pentane $30 \mathrm{~mL}$ ) was added to the deeped colored mixture and filtered. The resulting precipitate was washed with pentane $(3 \times 10$ $\mathrm{mL}$ ) and dried under vacum to afford Ni(II) complex (Ni-1) as a brown solid the $149 \mathrm{mg}$, $55 \%$ yield). The product was used without further purification. The complexwas stored in a nitrogen filled glove box at $30^{\circ} \mathrm{C} .{ }^{1} \mathbf{H}$ NMR $\left(500 \mathrm{MHz}, \mathrm{CDCb}{ }_{1}\right.$ A 8 ЉЙ j Óh $\mathrm{J}(\mathrm{q}$ $\mathrm{J}=14.5 \mathrm{~Hz}, 2 \mathrm{H}), 7.78(\mathrm{dJ}=7.8 \mathrm{~Hz}, 2 \mathrm{H}), 7.59(\mathrm{~d},=7.8 \mathrm{~Hz}, 2 \mathrm{H}), 7.55$ (s, 1H), 7.13 ( $\mathrm{d}=$ $29.6 \mathrm{~Hz}, 2 \mathrm{H}), 3.88(\mathrm{~s}, 3 \mathrm{H}), 1.42(\phi=35.3 \mathrm{~Hz}, 18 \mathrm{H})$. Spectral data was in agreement with the literature ${ }^{11}$. 


\subsection{Stoichiometric reactions with $\mathrm{Ni}-1$}<smiles>COC(=O)c1ccc([N+]2(Br)N3C=CC(Br)=CC3=C3C=C(C(C)(C)C)C=CN32)cc1</smiles>

$\mathrm{Ni}-1 \quad 1.0 \mathrm{eq}$<smiles>c1ccc(-c2cc(-c3ccccc3)c(-c3ccccc3)[n+](C3CCCCC3)c2)cc1</smiles>

$1.3 \mathrm{eq}$

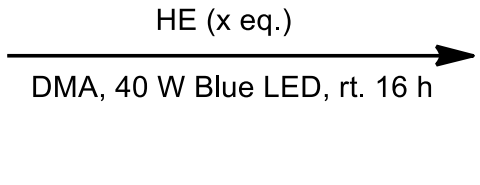

$$
\begin{aligned}
& x=2, G C \text { yield }=58 \% \\
& x=0, G C \text { yield }=0 \%
\end{aligned}
$$

To an ovendried $5 \mathrm{~mL}$ vial equipped with a magnetic stir bar was added pyridinium salts $(0.13 \mathrm{mmol})$ and HE (x equiv). The vial was transferred to a $\mathrm{N}$-filled glovebox. Ni-1 $(0.1$ mmol) was added to the vial, and the mixture was then dissolved in $1.0 \mathrm{~mL}$ DMA. The vial was tightly sealed and removed from the glovebox.he reaction was allowed to stir under $40 \mathrm{~W}$ blue LED irradiation for $16 \mathrm{~h}$ with fans to maintain the temperature below $30{ }^{\circ} \mathrm{C}$ (the vial was placed about $3 \mathrm{~mm}$ from the LEDs, see experimental setuffter that, the reaction was diluted with $1 \mathrm{~mL}$ EtOAc, and them-tridecane $(12 \mathrm{mg})$ as an internal

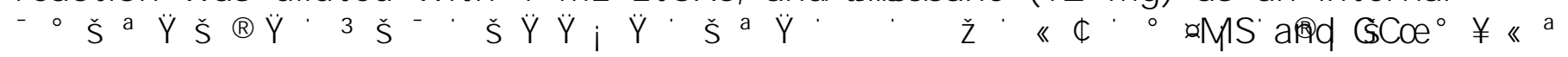
analysis.

\subsection{Catalytic competence of $\mathrm{Ni}-1$}<smiles>COc1ccc(Br)cc1</smiles><smiles>c1ccc(-c2cc(-c3ccccc3)c(-c3ccccc3)[n+](-c3ccccc3)c2)cc1</smiles>

$1.3 \mathrm{eq}$

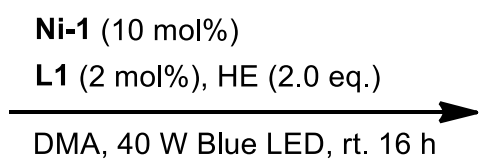

DMA, $40 \mathrm{~W}$ Blue LED, rt. $16 \mathrm{~h}$<smiles>COC(=O)c1ccc(C2CCCCC2)cc1</smiles>

GC yield $=76 \%$

To an ovendried $5 \mathrm{~mL}$ vial equipped with a magnetic stimar was added pyridinium salts (0.13 mmol), HE 2.0 equiv) and $\mathbf{L 1}(2 \mathrm{~mol} \%)$. The vial was transferred to a $\mathrm{N}$-filled glovebox.Ni-1 $(0.01 \mathrm{mmol})$ was added to the vial, anthe mixture was then dissolved in 1.0 mL DMA. The vial was tightly sealed and removficbm the glovebox. The reaction was allowed to stir under $40 \mathrm{~W}$ blue LED irradiation for $16 \mathrm{~h}$ with fans to maintain the temperature below $30{ }^{\circ} \mathrm{C}$ (the vial was placed about $3 \mathrm{~mm}$ from the LEDs, see experimental setup). After that, the reaction was diluted with $1 \mathrm{~mL}$ EtOAc, and them-

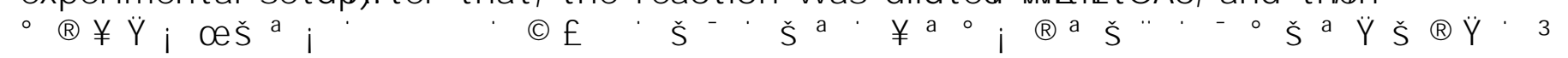
was taken out for GEMS and GC analysis.

\subsection{UV/Vis Absorption spectra}

The UV/Vis absorption spectra ofDMAsolutions of Hantzsch ester $(0 . \theta \mathrm{M})$, pyridinium salts $(0.0325 \mathrm{M})$, a mixture of $\mathrm{HE}(0.6 \mathrm{M})$ and pyridinium salts $(0.023 \mathrm{M}$ were recorded in $1 \mathrm{~cm}$ path quartz cuvettes using a UV800PC spectrophotometerA mixture of 1 and 3 displayed a significant redshift in absorbance, confirming formaton of the postulated EDA complex 

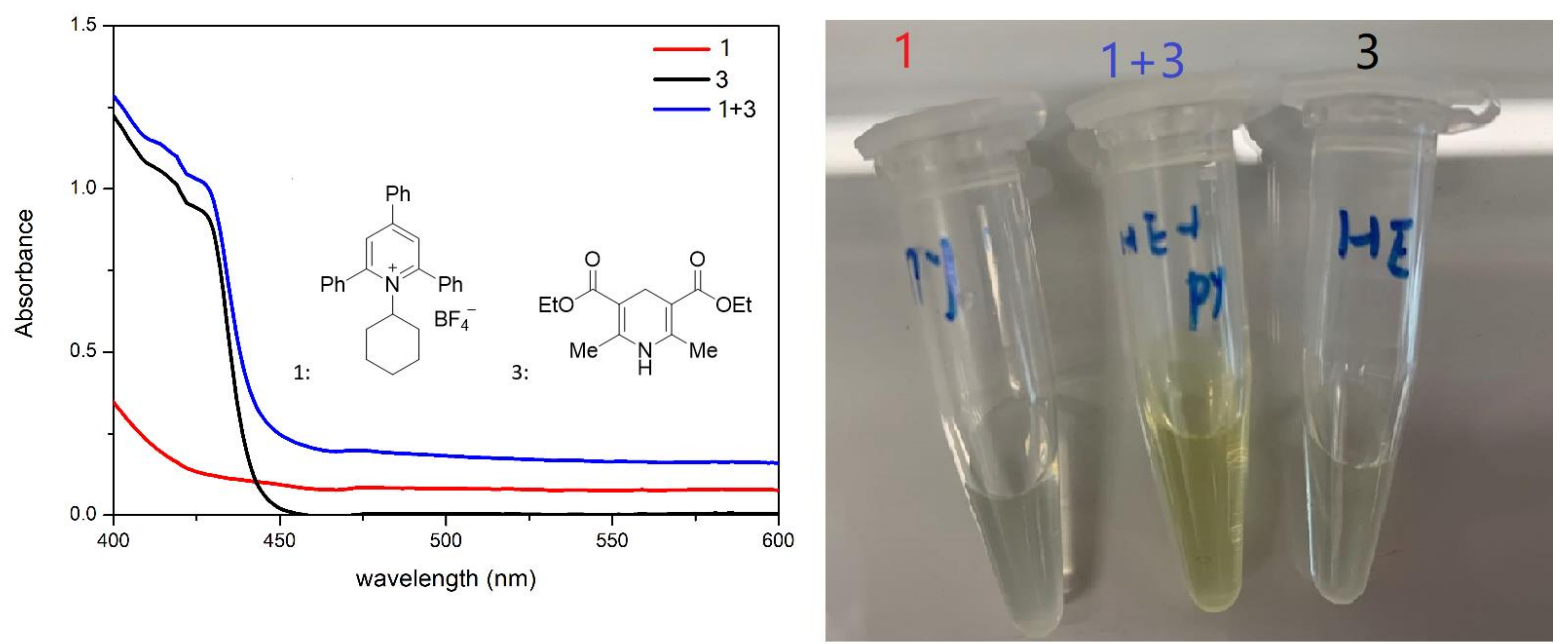

Figure S1 show the UV/Vis absorption spectra of DMA solutions of secondary alkyl pyridinium 1 and Hantzsch ester.

\section{References}

1. Basch,C. H: Liao,J; Xu,J; Piane,J. J.Watson,M. P.J . Am. Chem. S \&o17, 139, 5313.

2. Plunkett, S; Basch,C. H; Santana,S. O,.Watson, M. P.J. Am. Chem. S\&2019, 141, 2257.

3. Klauck,F. J. R. J ames,M. J;:Glonius, F.Angew. Chem. Int. Ed017, 56, 12336.

4. Sun,Z; Romano,C; Martin, R.J . Am. Chem. S\&019, 141, 16197.

5. Zhang,X.; MacMillan, D.W. CJ. Am. Chem. S\&016, 138, 13862.

6. Bhonde,V. R; OÏNeill, B. T; Buchwald,S. LAngew. Chem. Int. Ed016, 55, 1849.

7. Yi,J; Badir, S. O,.Kammer,L. M; Ribagorda,M.; Molander, G. A.Org. Lett 2019, 21, 3346.

8. Zhao, S.; Gensch, T.; Murray, B.; Niemeyer, Z. L.; Sigman, M. S.; Bisco€,chinBe 2018, 362, 670 .

9. Martin-Montero, R.; Reddy Yatham, V.; Y in, H.; Davies, J .; MartiD,rR.Lett. 2019, 21, 2947.

10. Ni, S.; Li, C. X.; Mao, Y.; Hang,Wang, Y.; Yan, H.; PanS đi. Adv2019, 5: eaaw9516

11. L. Guo, F. Song, S. Zhu, HLLChu,Nat. Commun 2018, 9, 4543. 


\section{NMR Spectra}

1H NMR (500 MHz, CDG) for $4 \mathbf{a}$

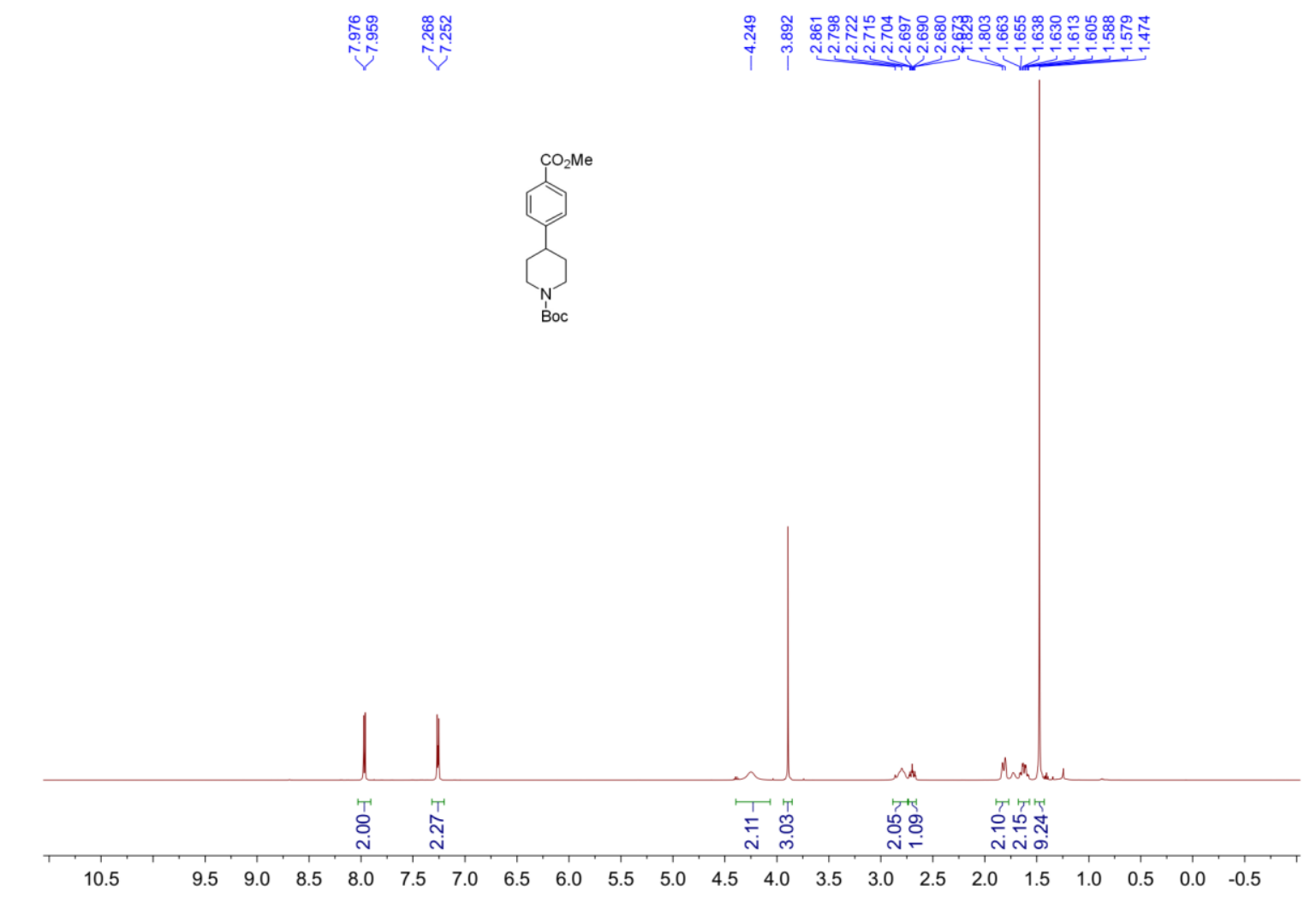

13C NMR (126 MHz, CDG) for 4a

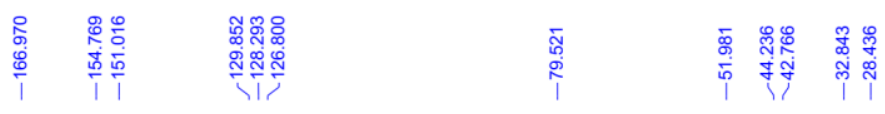
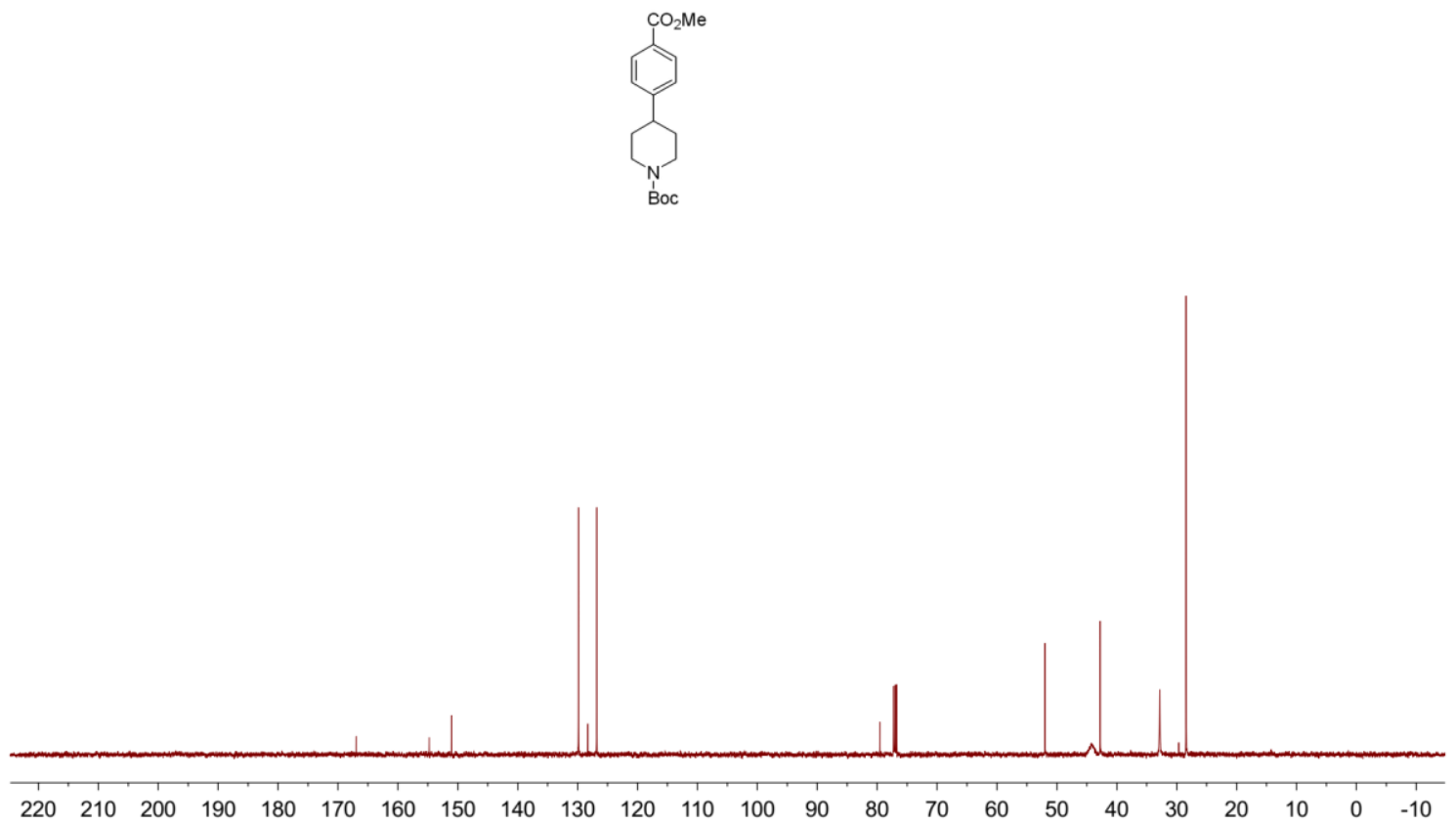
1H NMR (500 MHz, CDG) for 4c

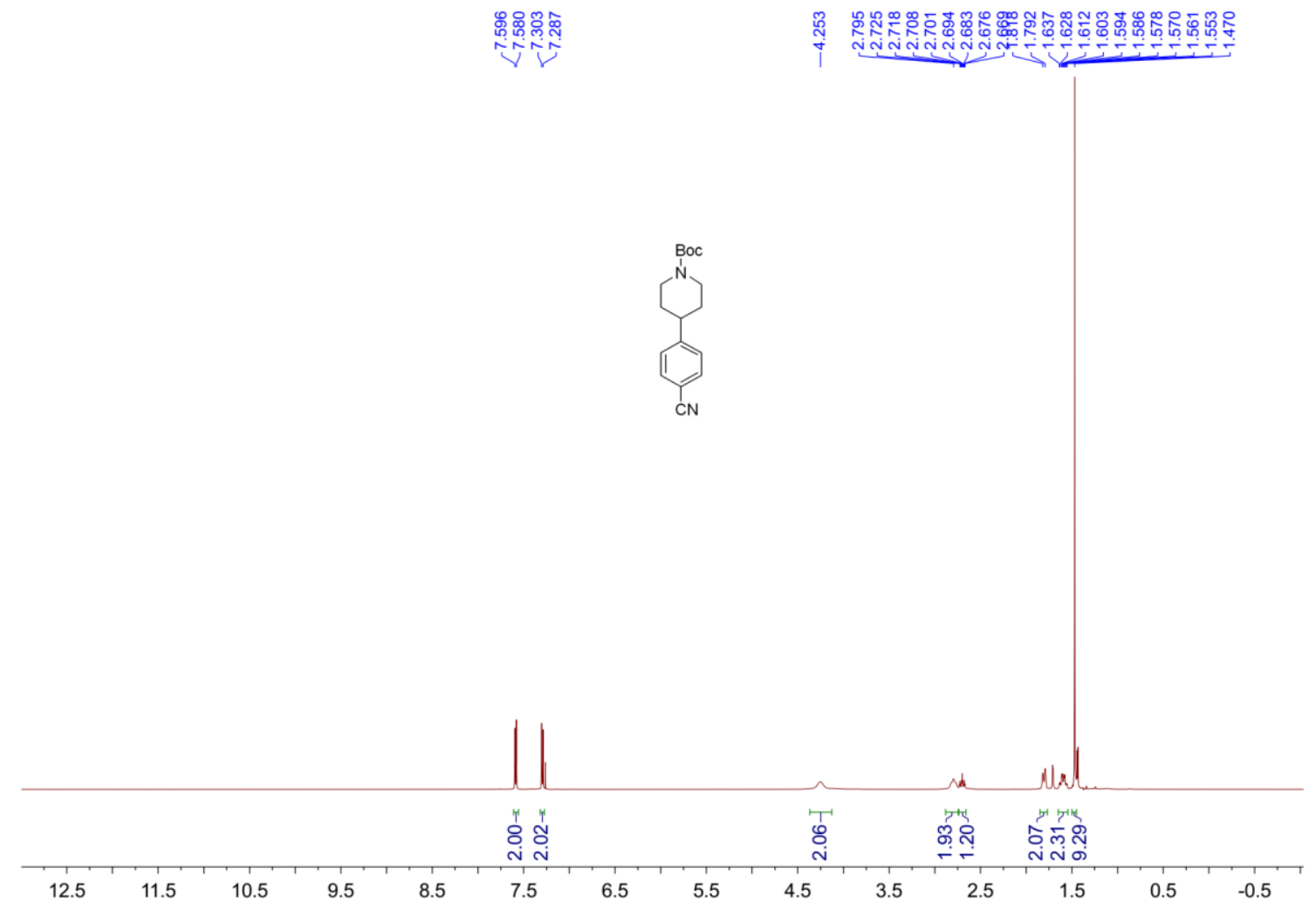

13C NMR (126 MHz, CDG) for 4c

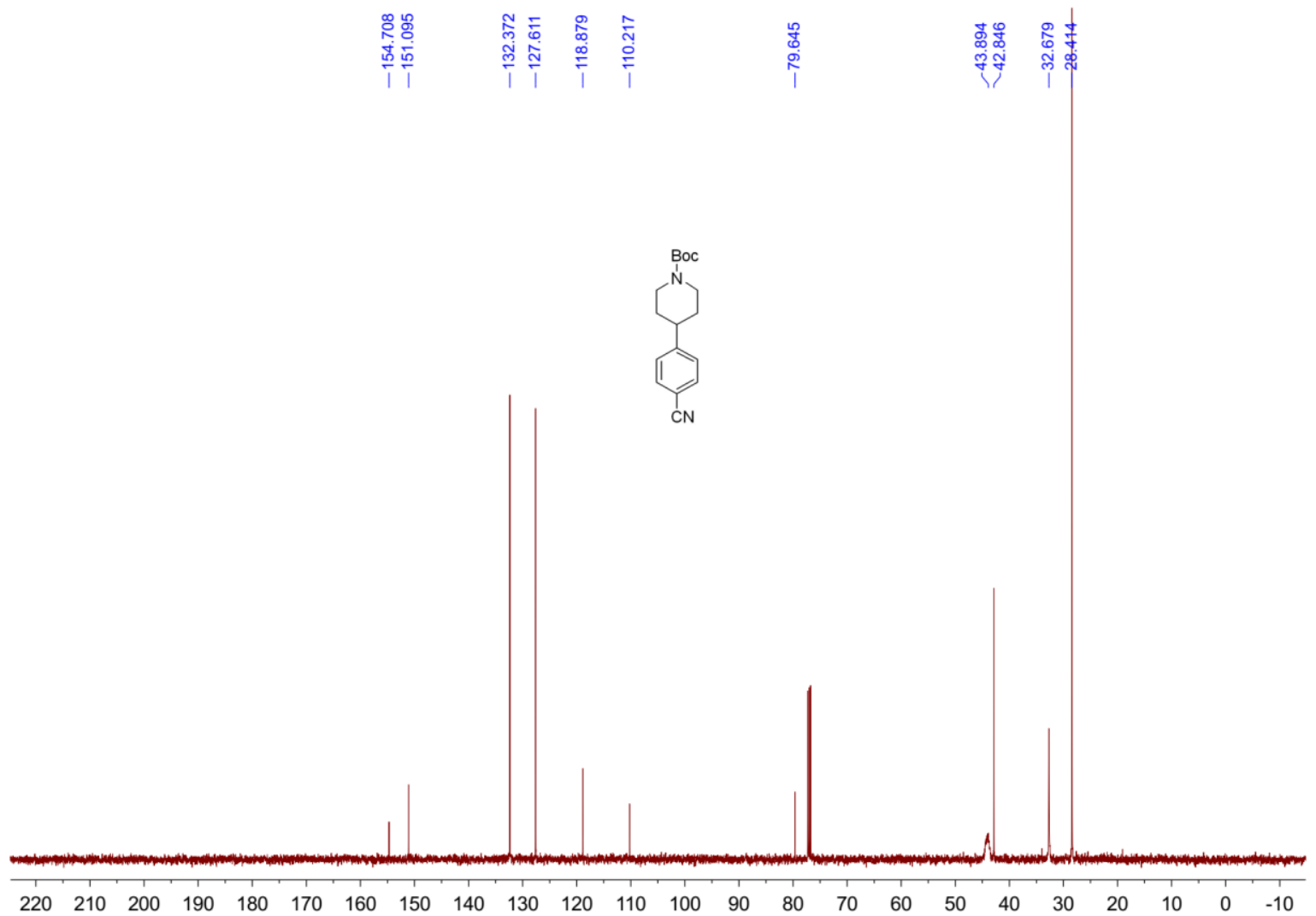


1H NMR (500 MHz, CDG) for 4d

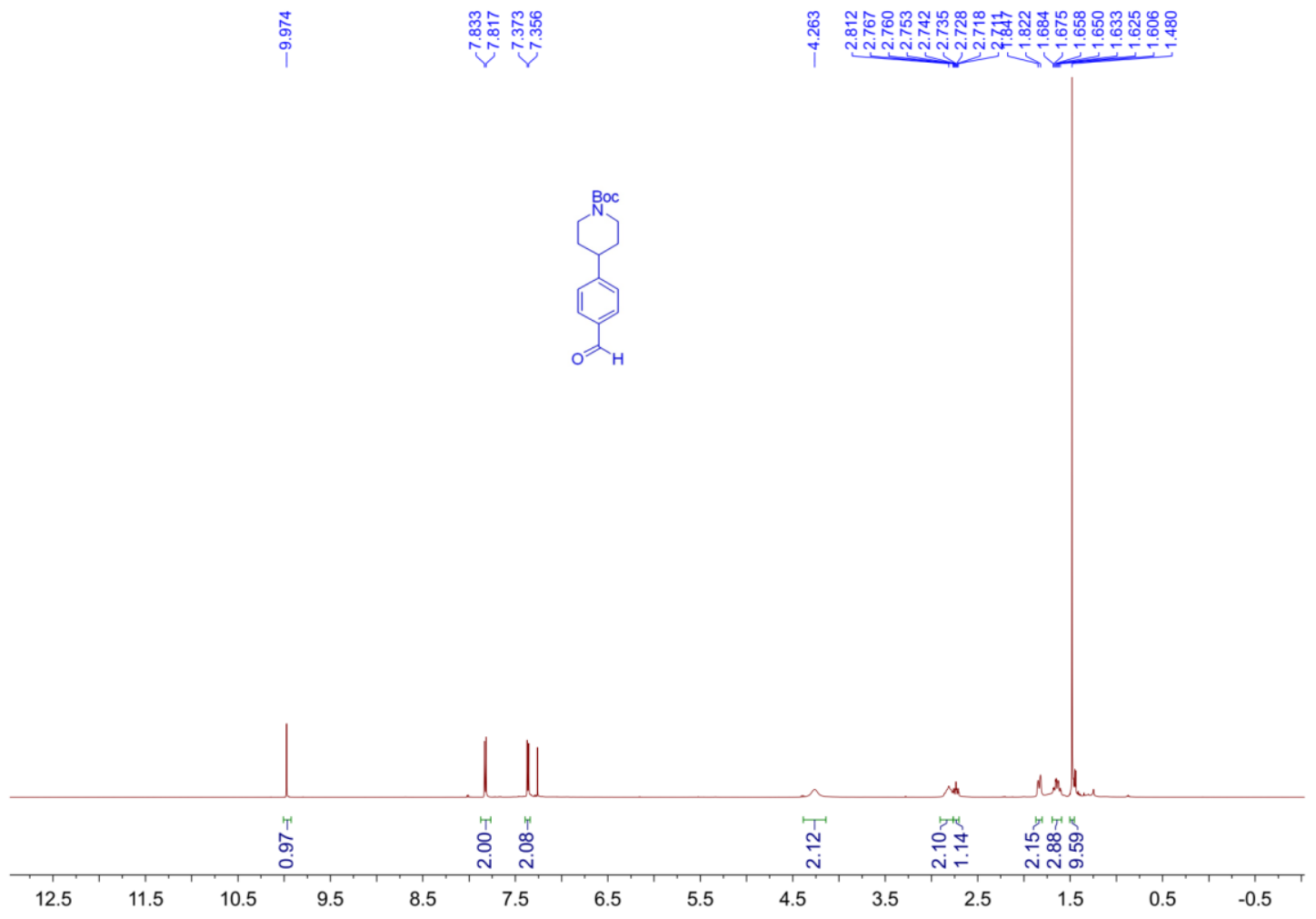

13C NMR (126 MHz, CDG) for 4d

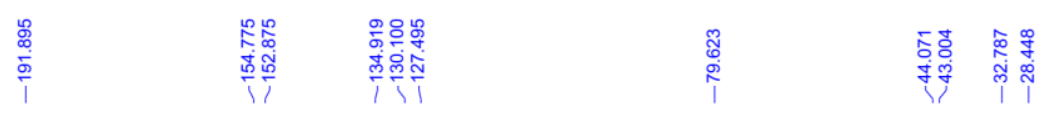
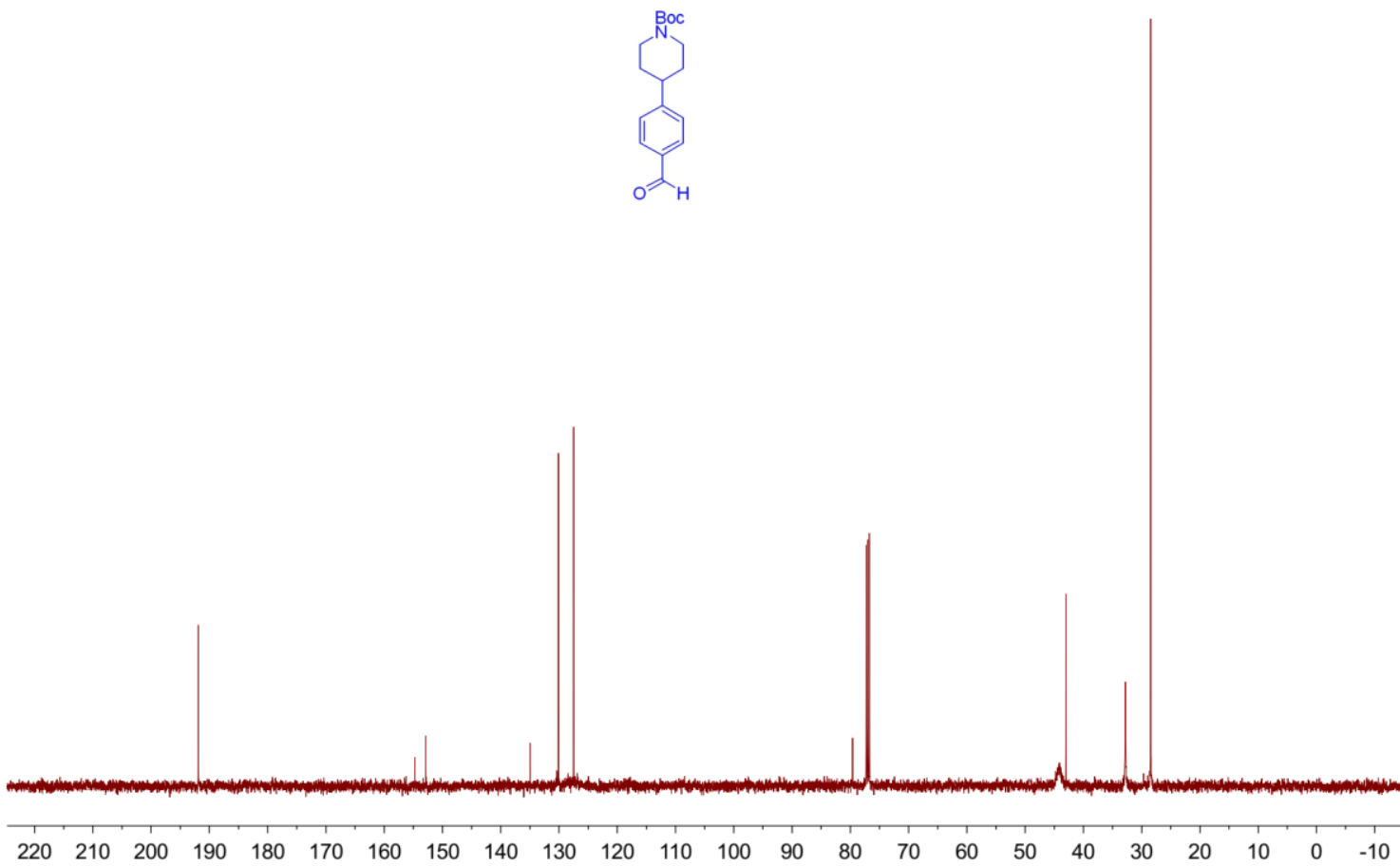
1H NMR (500 MHz, CDG) for $4 \mathbf{e}$

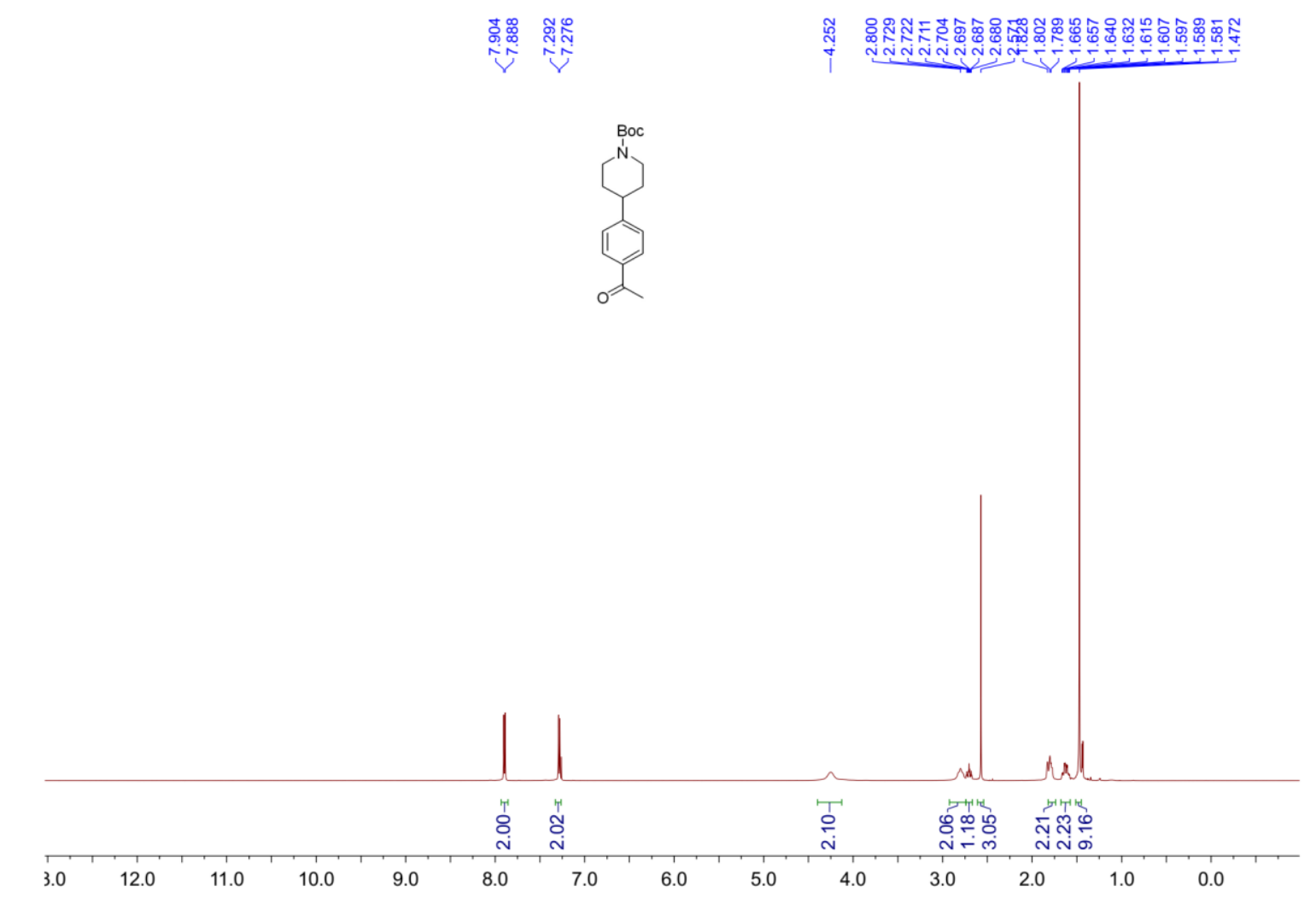

13C NMR (126 MHz, CDG) for 4e

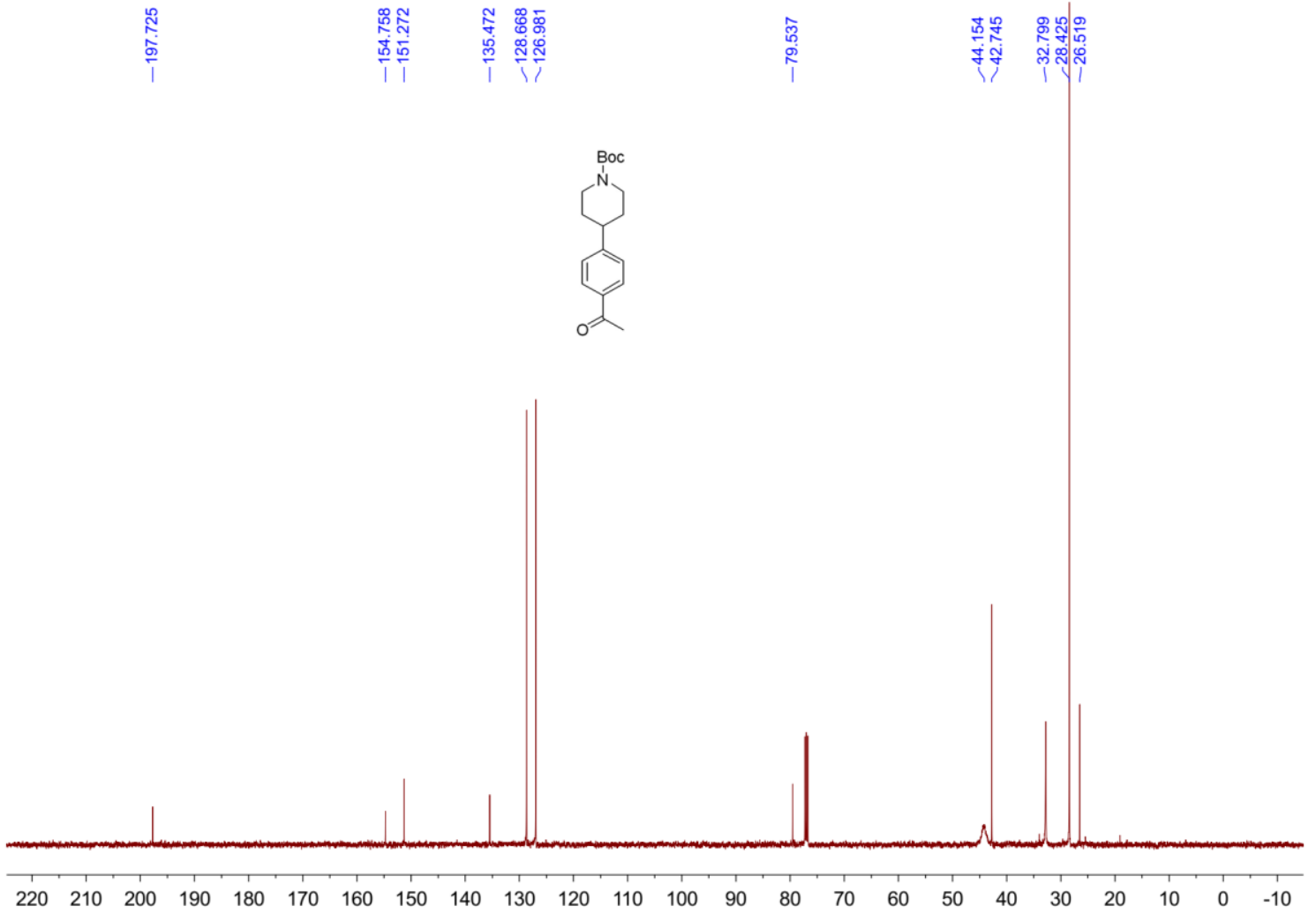


1H NMR (500 MHz, CDG) for $\mathbf{4 f}$

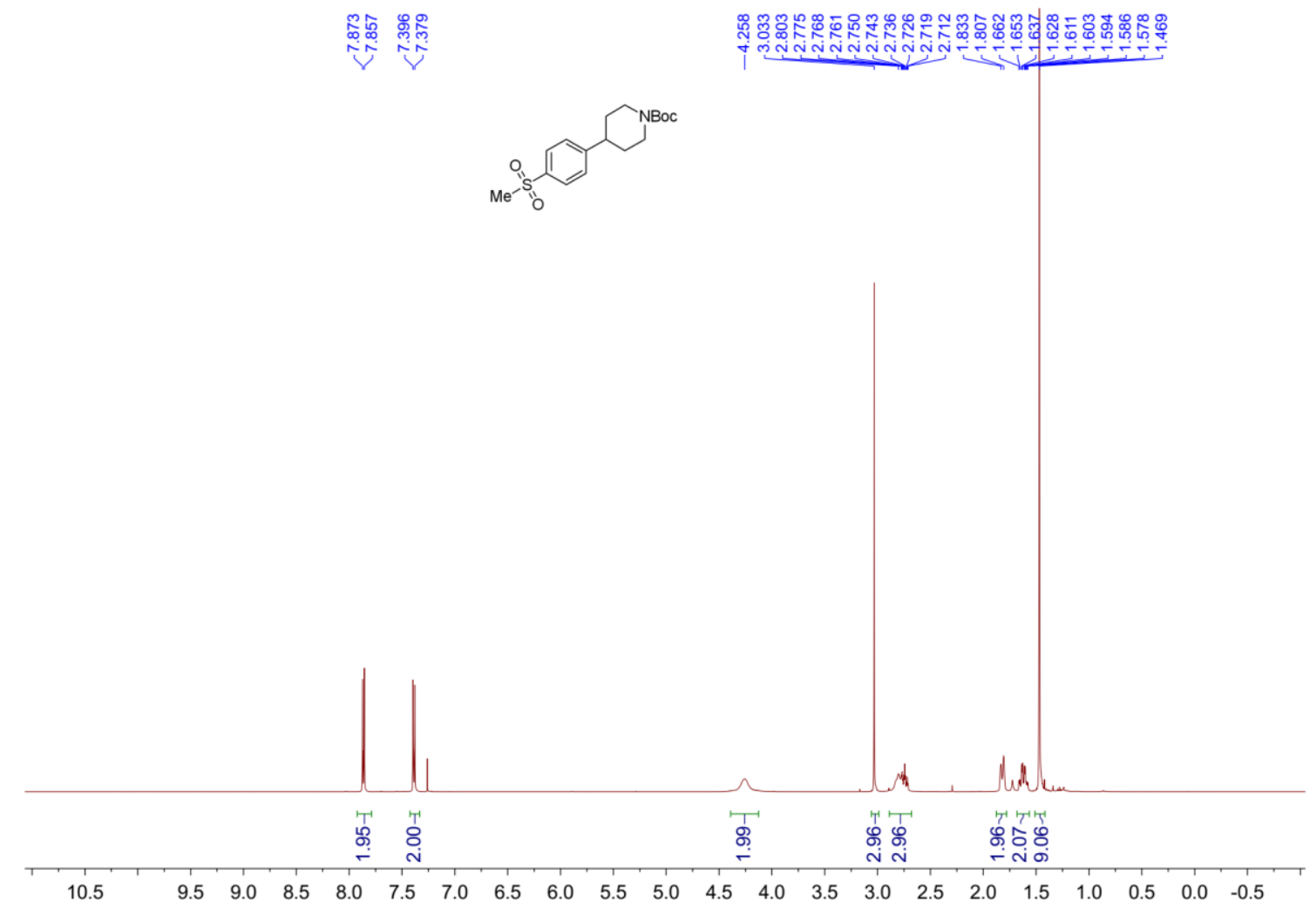

${ }^{13}$ C NMR (126 MHz, CDG) for $\mathbf{4 f}$
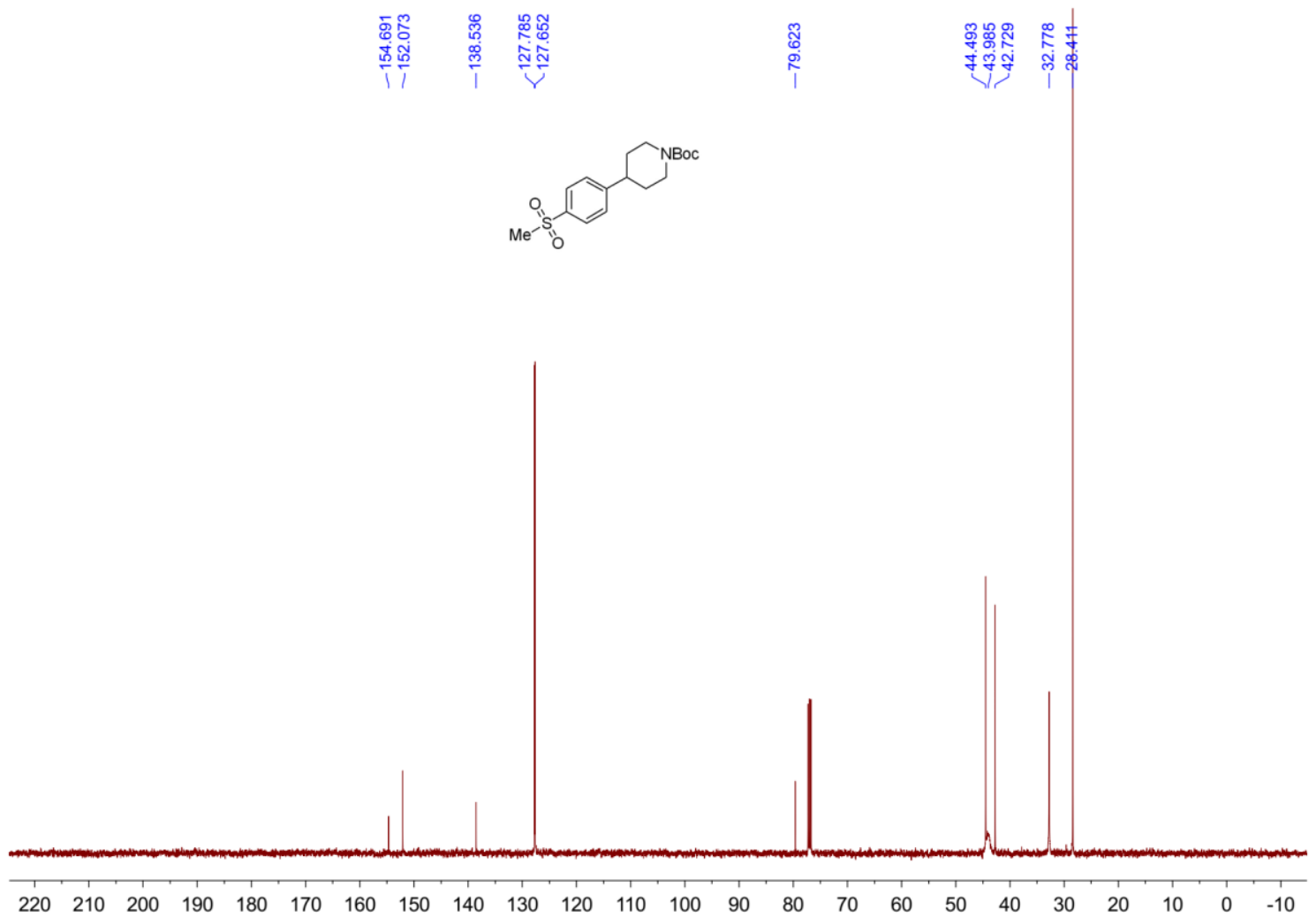
1H NMR (500 MHz, CDG) for 4g

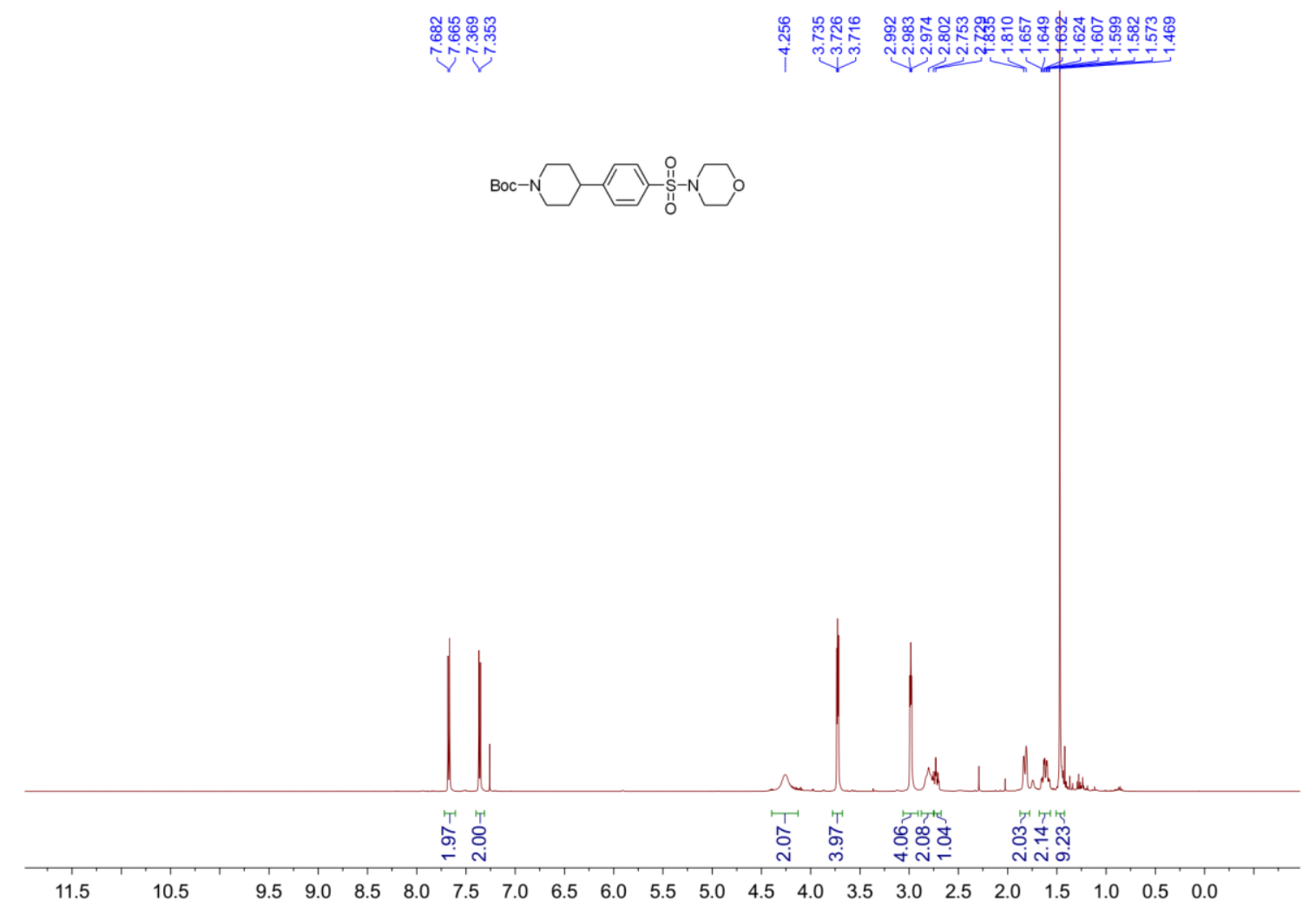

13C NMR (126 MHz, CDG) for $\mathbf{4 g}$
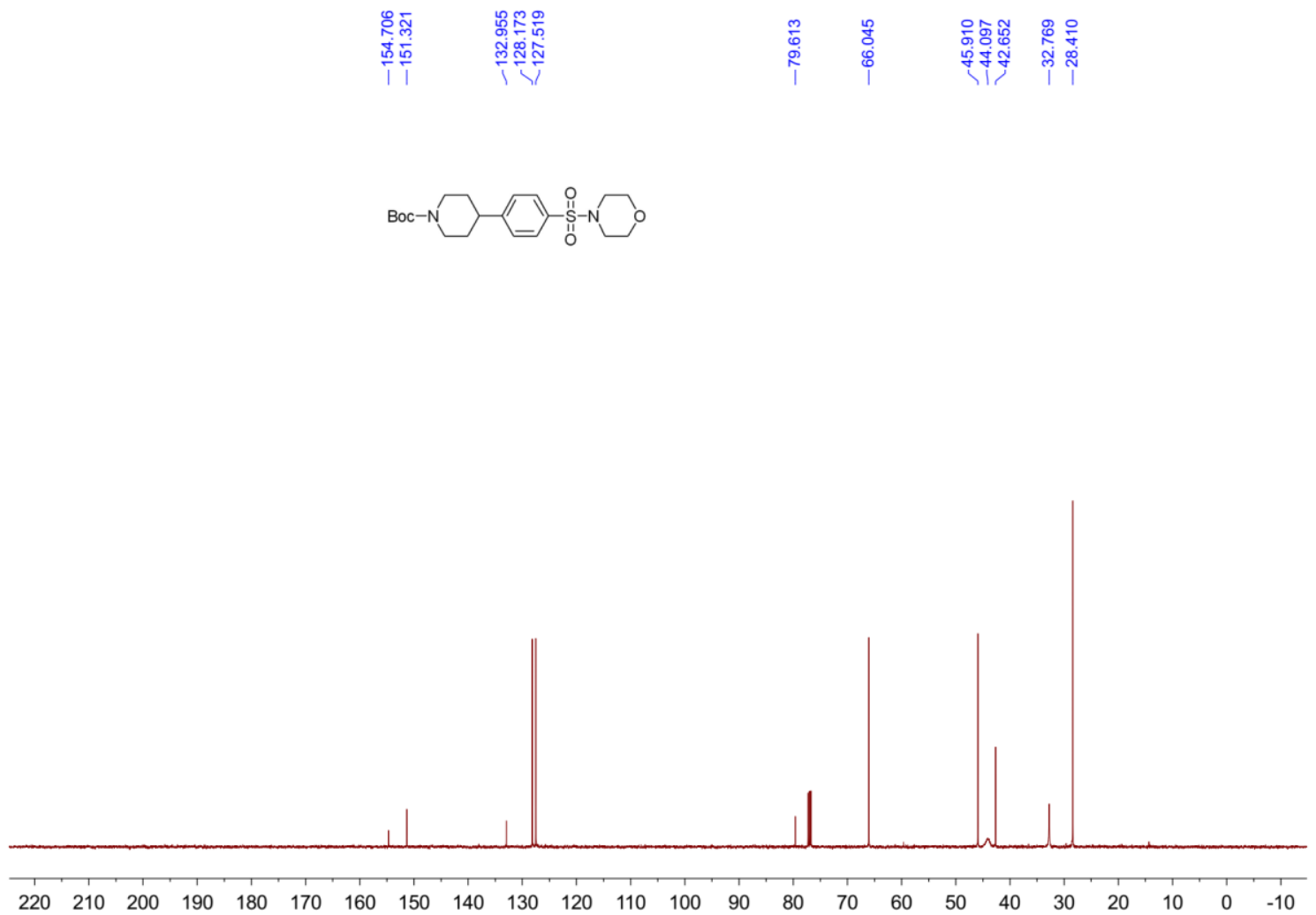
1H NMR (500 MHz, CDG) for $\mathbf{4 h}$

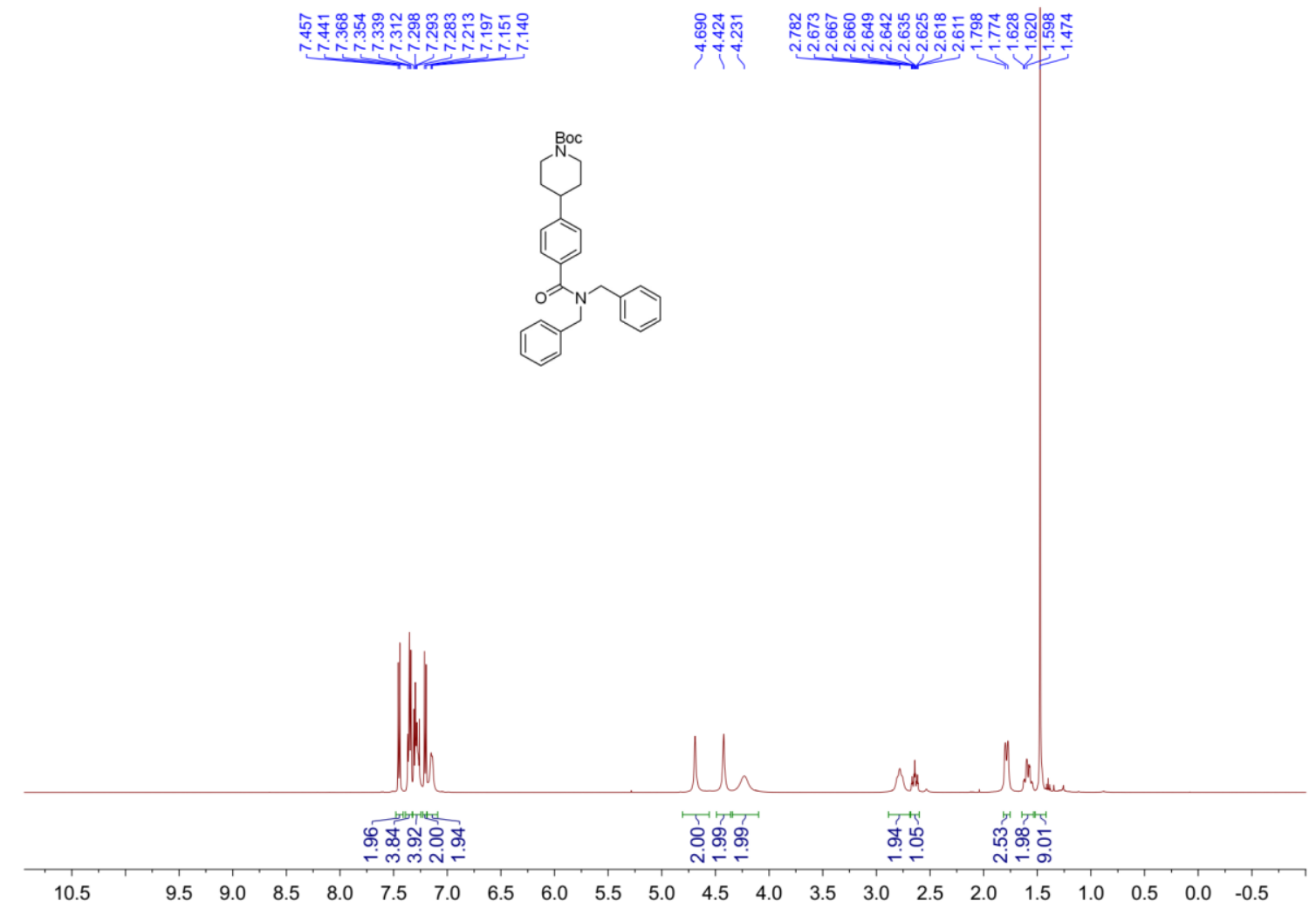

13C NMR (126 MHz, CDG) for $\mathbf{4 h}$

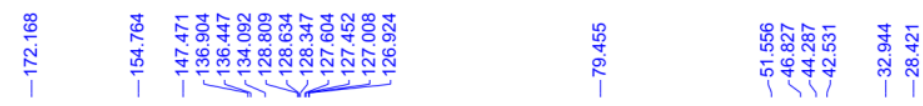

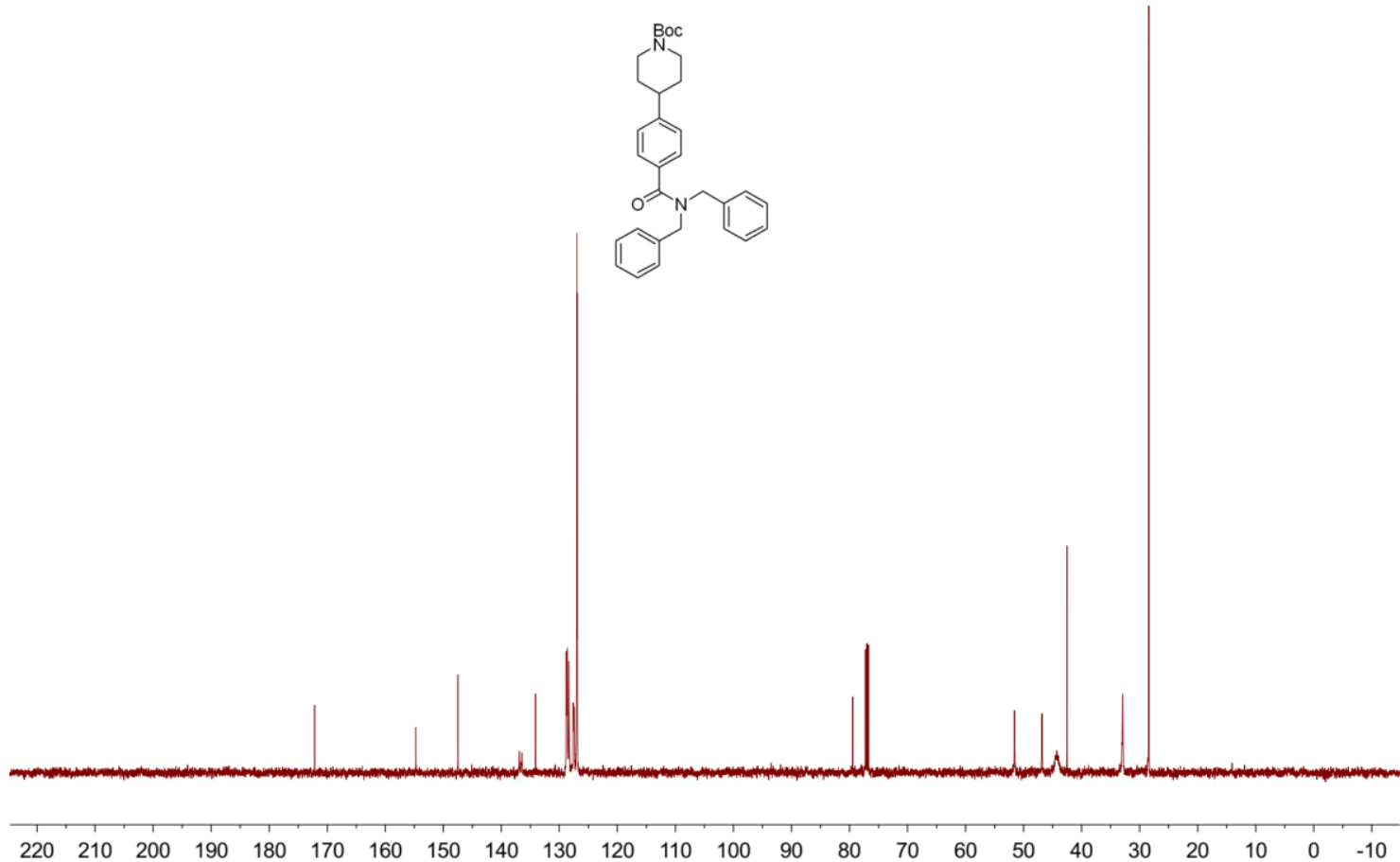


1H NMR (500 MHz, CDG) for 4i

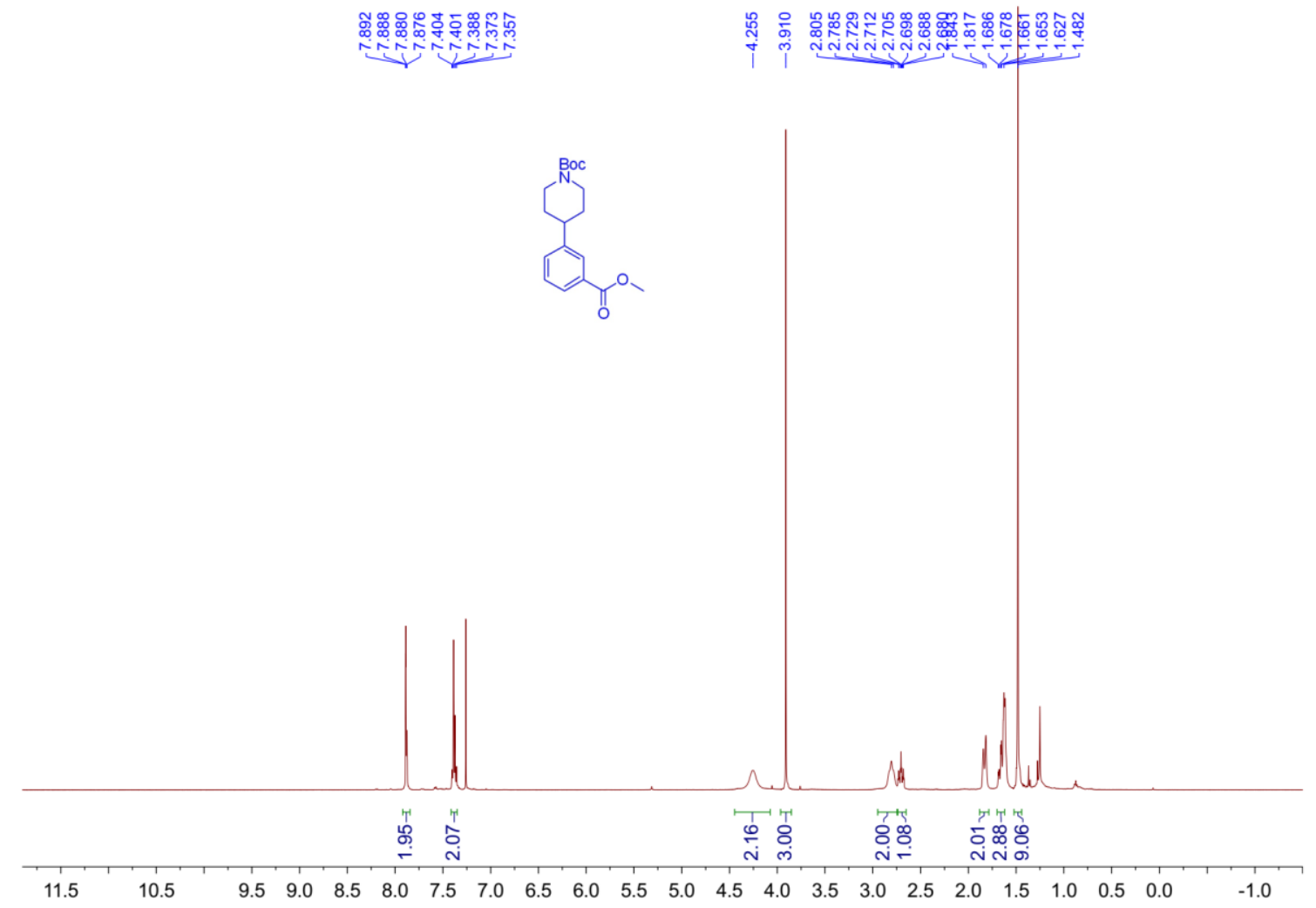

13C NMR (126 MHz, CDG) for $\mathbf{4 i}$

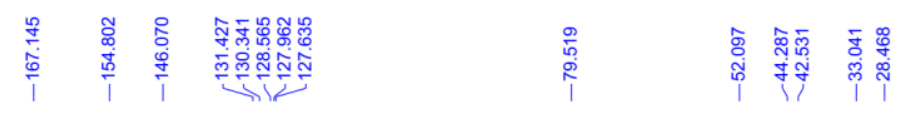<smiles>COC(=O)c1cccc(C2CC[NH2+]CC2)c1</smiles>

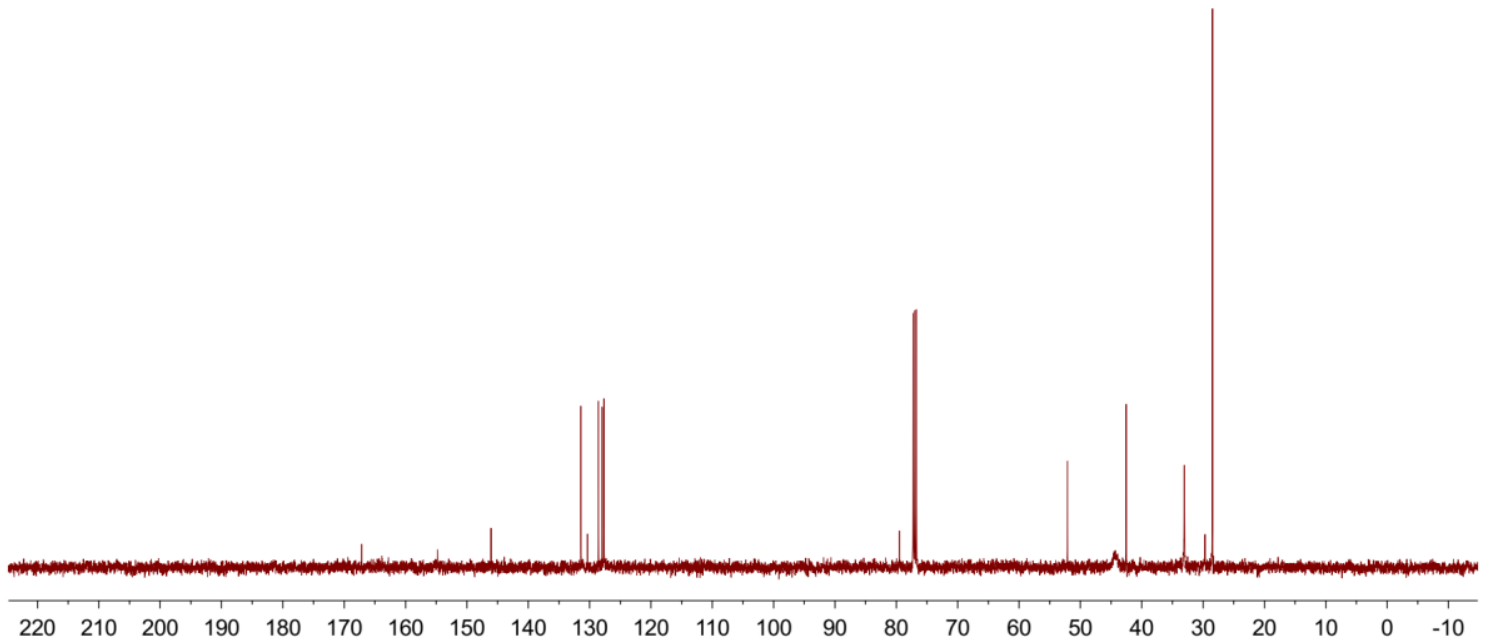


1H NMR (500 MHz, CDG) for 4j

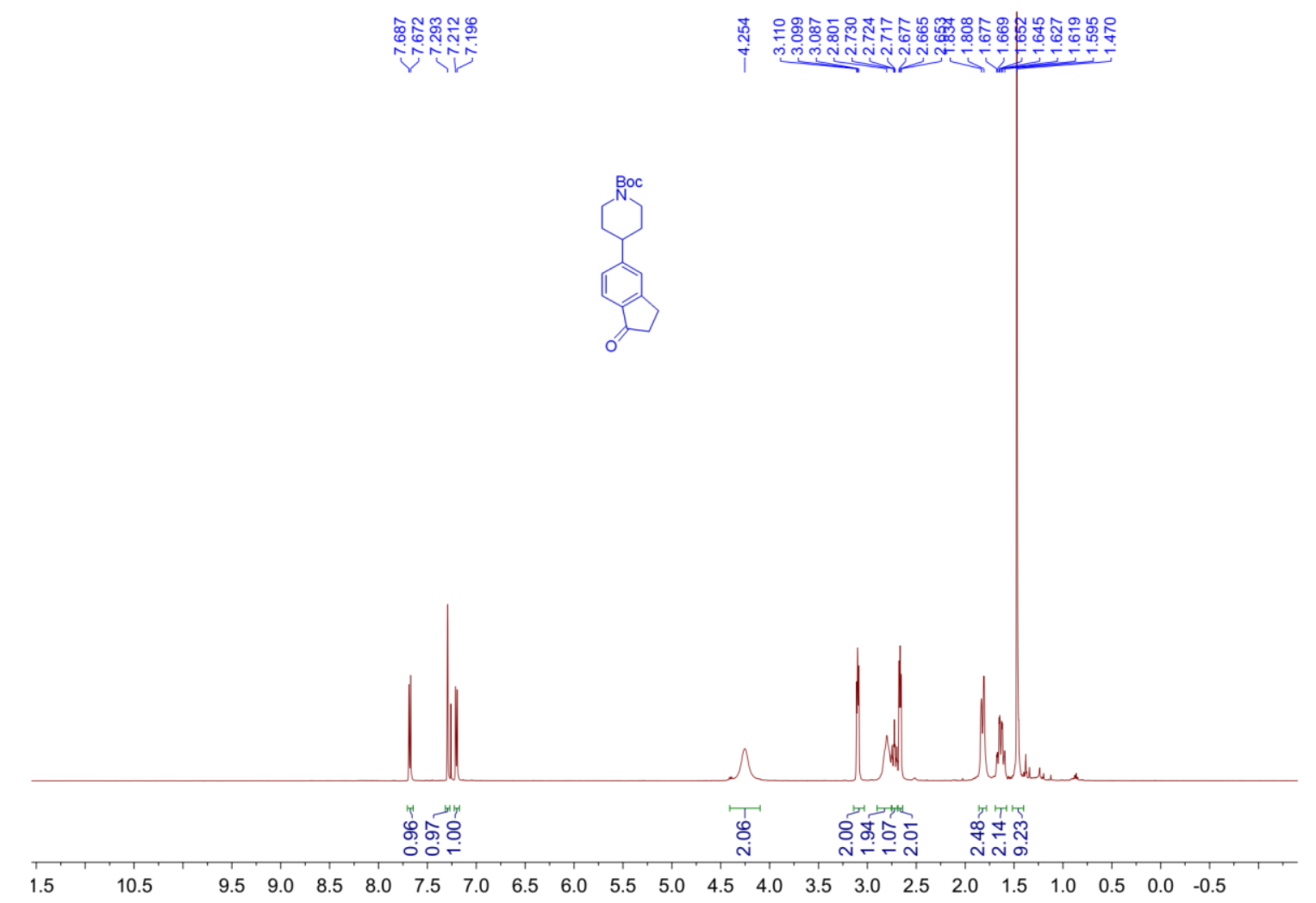

13C NMR (126 MHz, CDG) for 4j

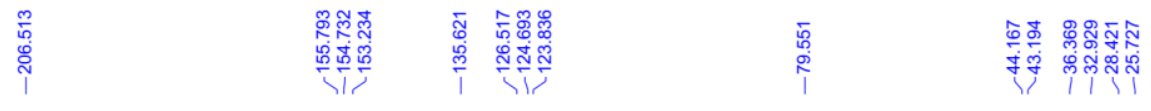

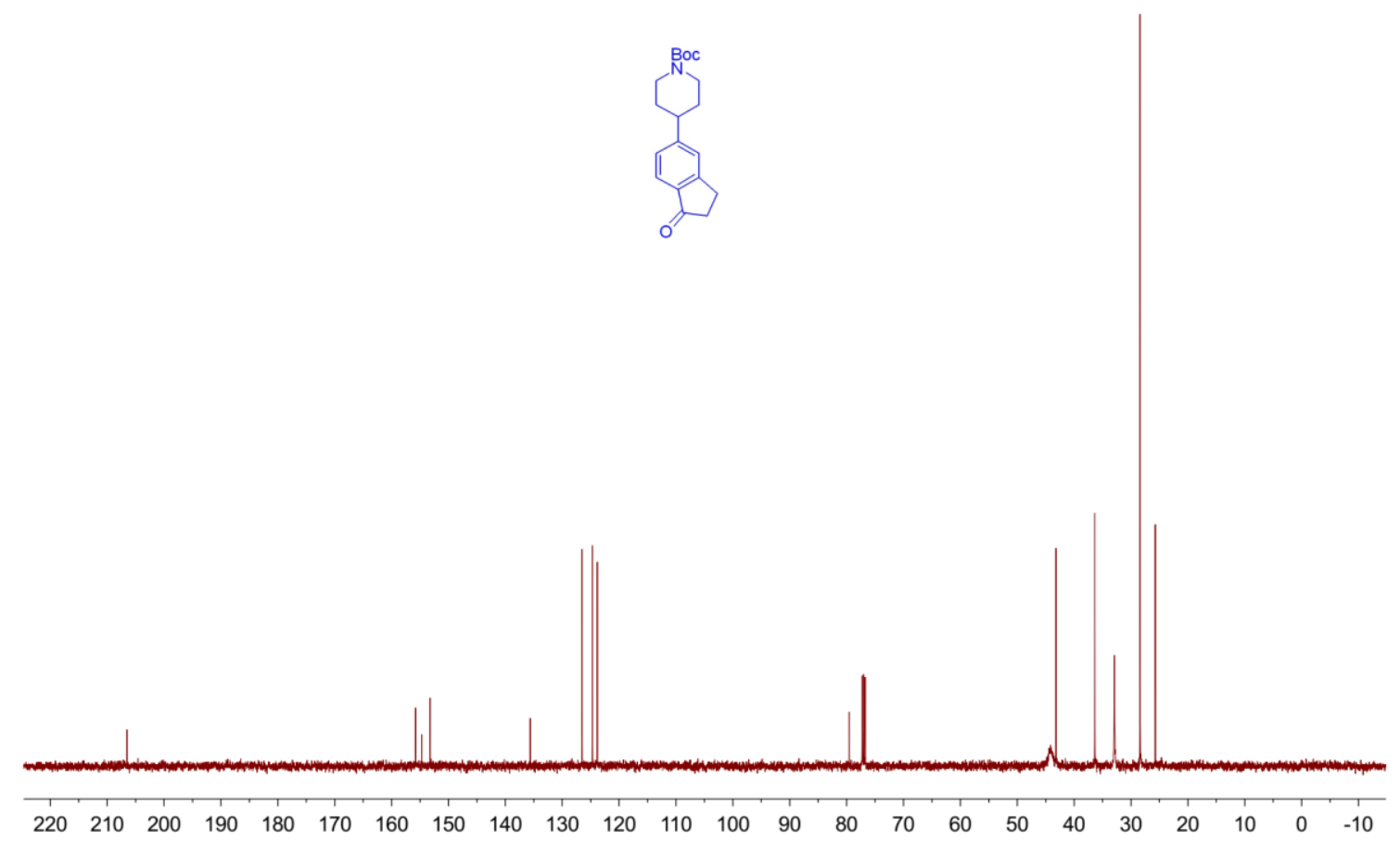


1H NMR (500 MHz, CDG) for $\mathbf{4 k}$

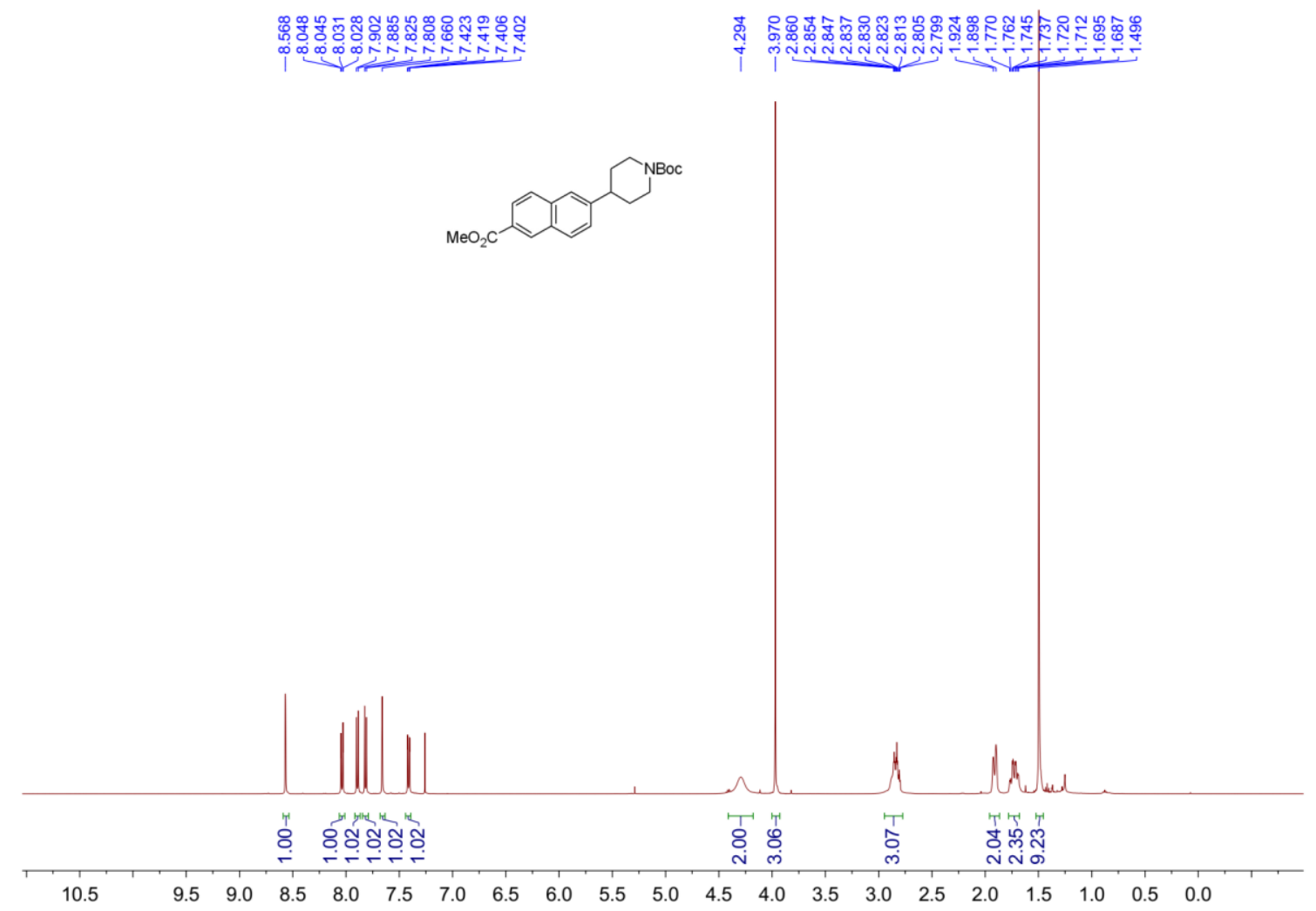

13C NMR (126 MHz, CDG) for $\mathbf{4 k}$

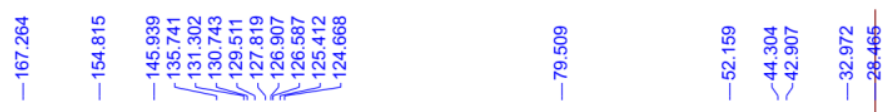
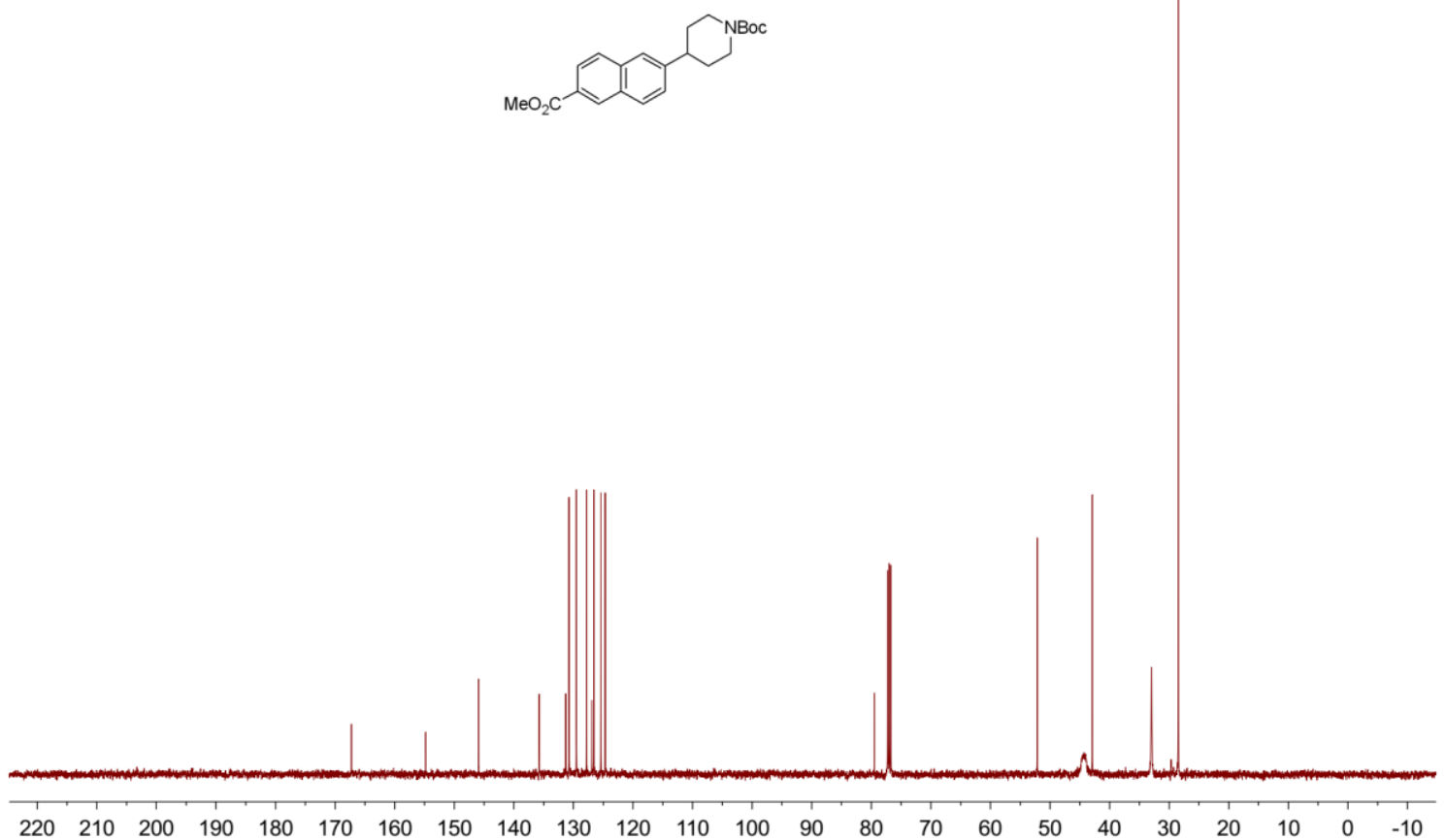
1H NMR (500 MHz, CDG) for $4 \mathbf{l}$

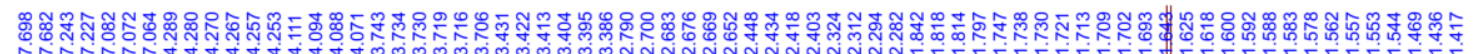

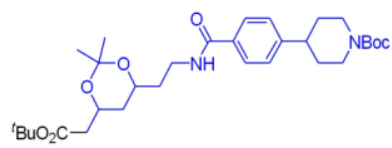

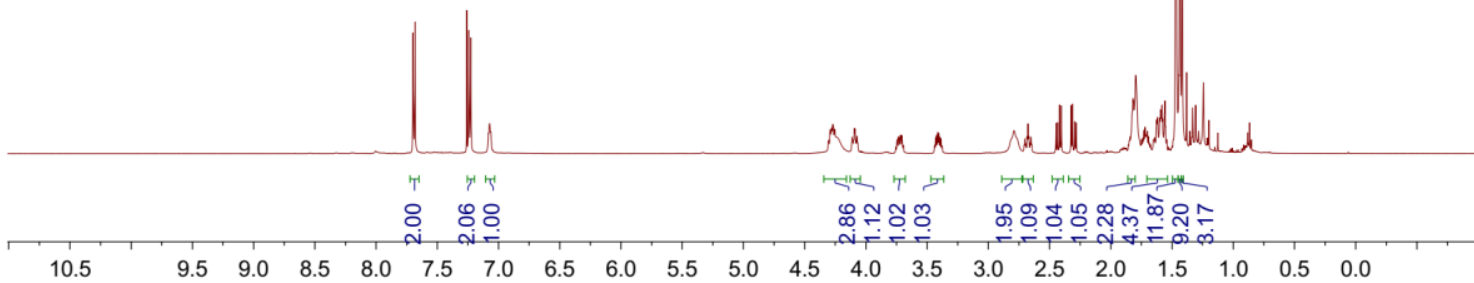

${ }^{13} \mathbf{C}$ NMR (126 MHz, CDG) for $4 \mathbf{l}$

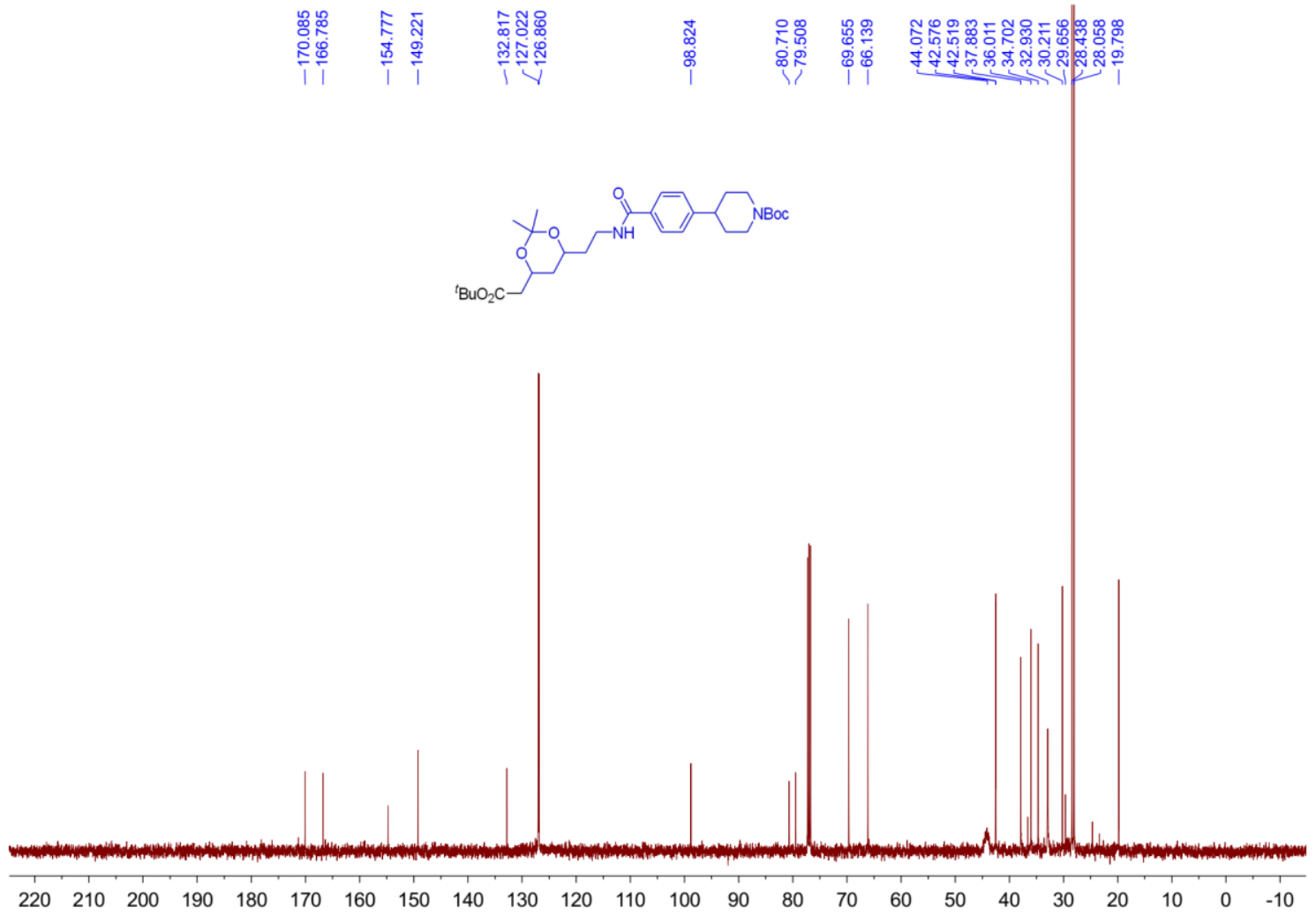


1H NMR (500 MHz, CDG) for $\mathbf{4 n - \boldsymbol { \alpha }}$

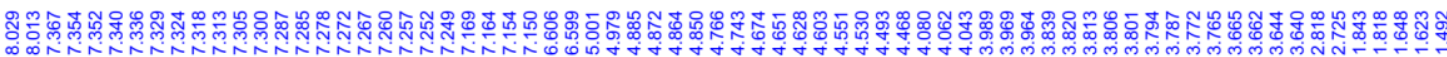

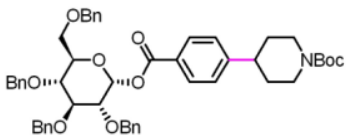

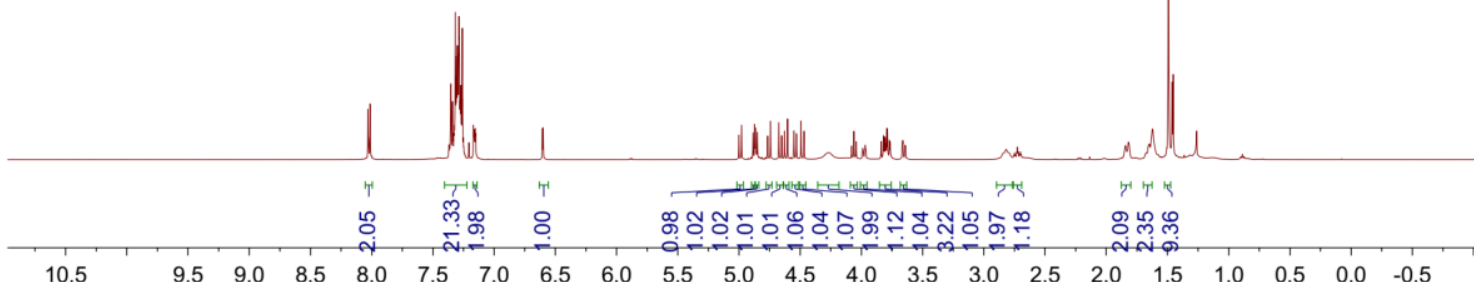

13C NMR (126 MHz, CDG) for $\mathbf{4 n - \boldsymbol { \alpha }}$

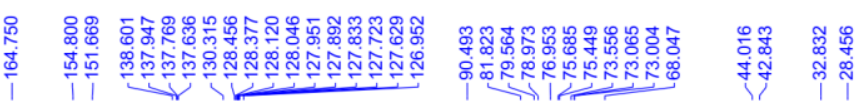
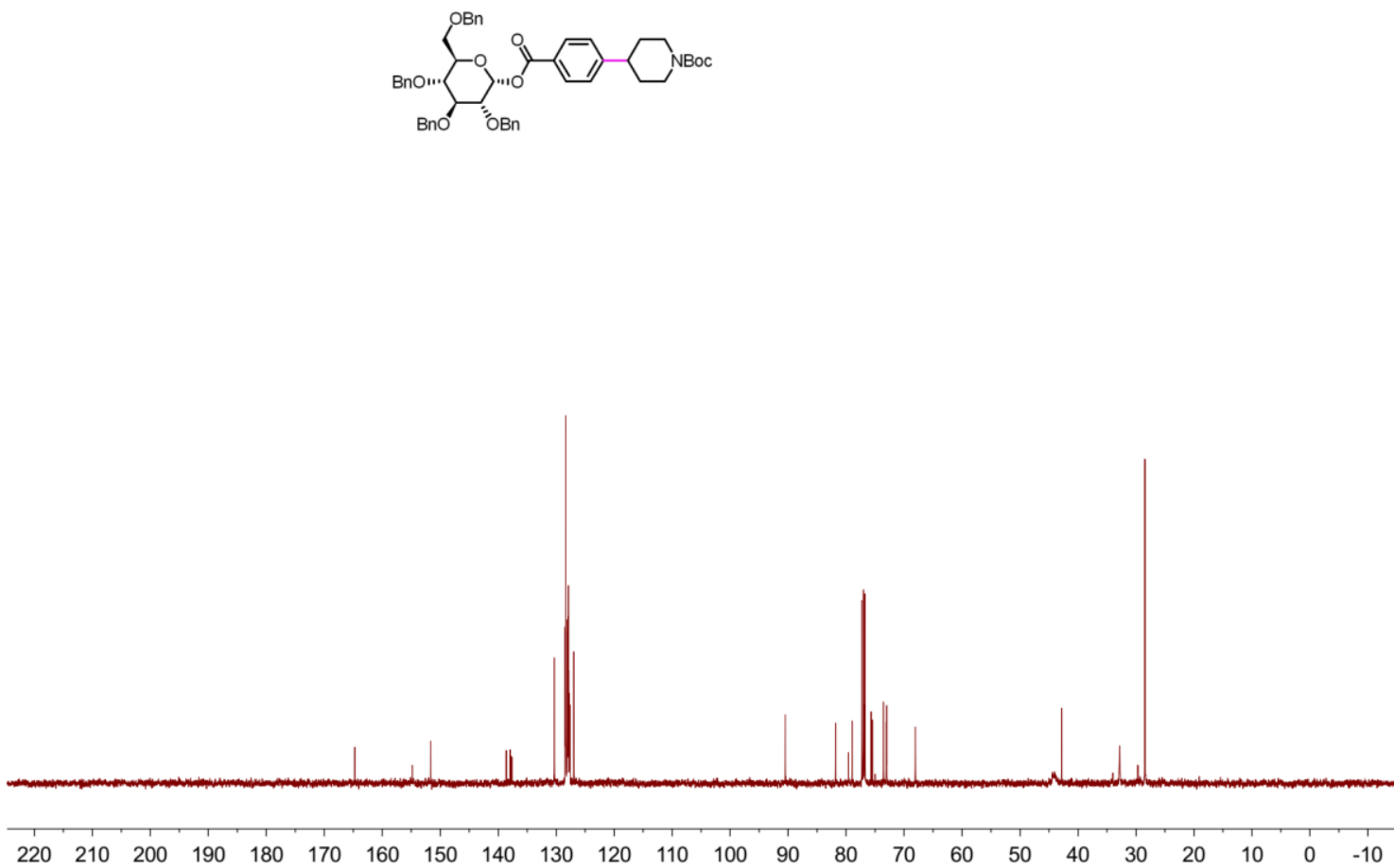
1H NMR (500 MHz, CDG) for $\mathbf{4 n - \boldsymbol { \beta }}$

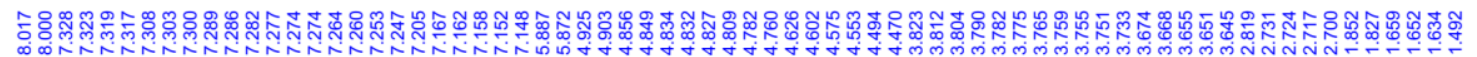

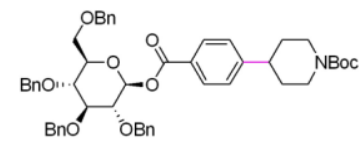

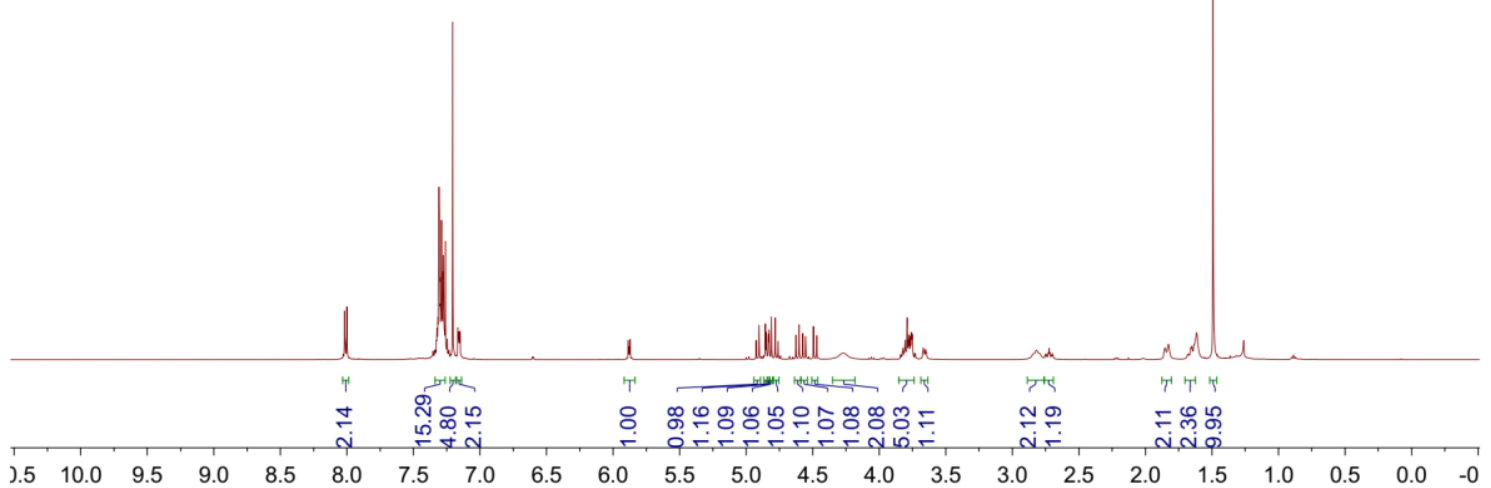

${ }^{13} \mathbf{C}$ NMR (126 MHz, CDG) for $\mathbf{4 n - \beta}$

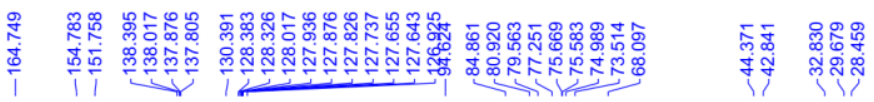

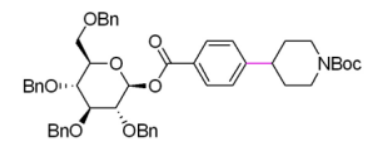

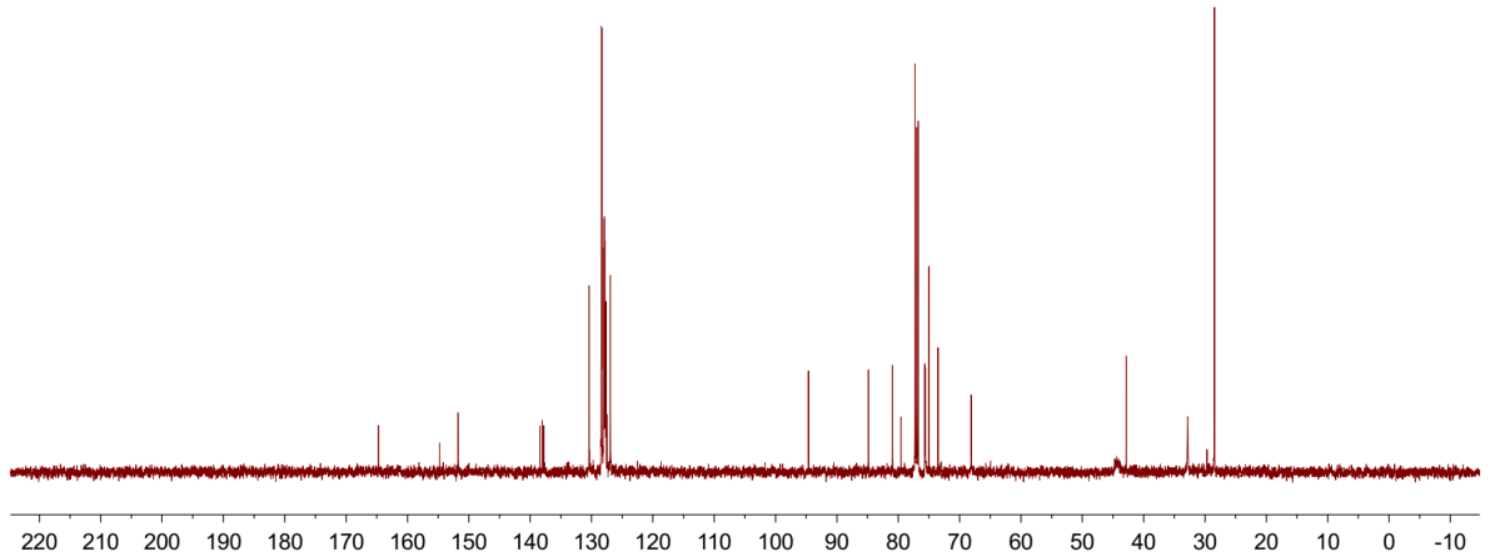


1H NMR (500 MHz, CDG) for 4 o

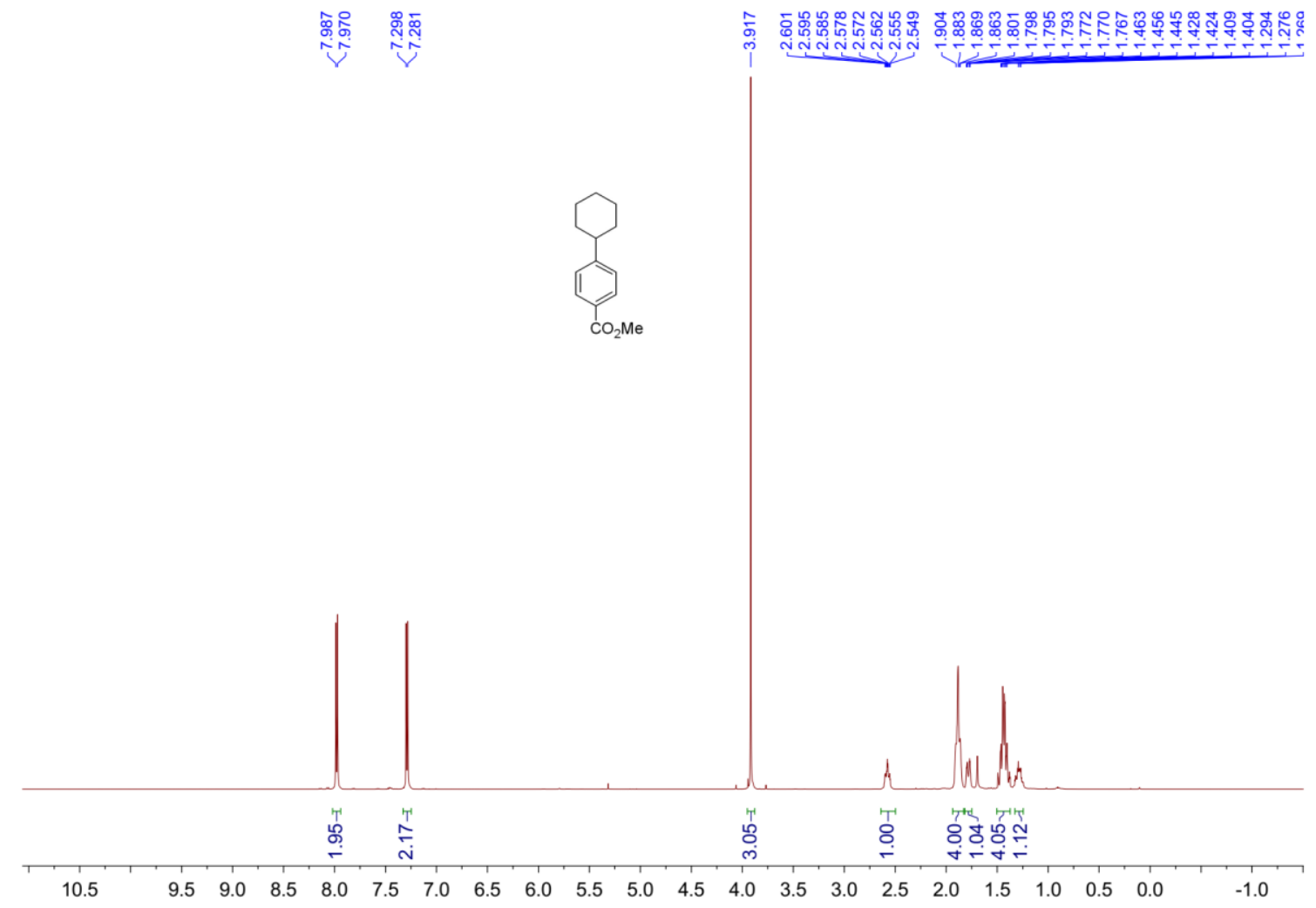
13C NMR (126 MHz, CDG) for 40

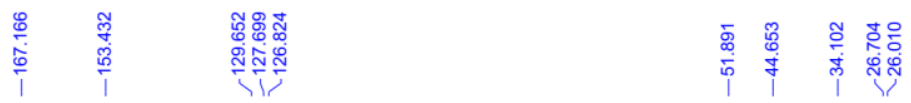

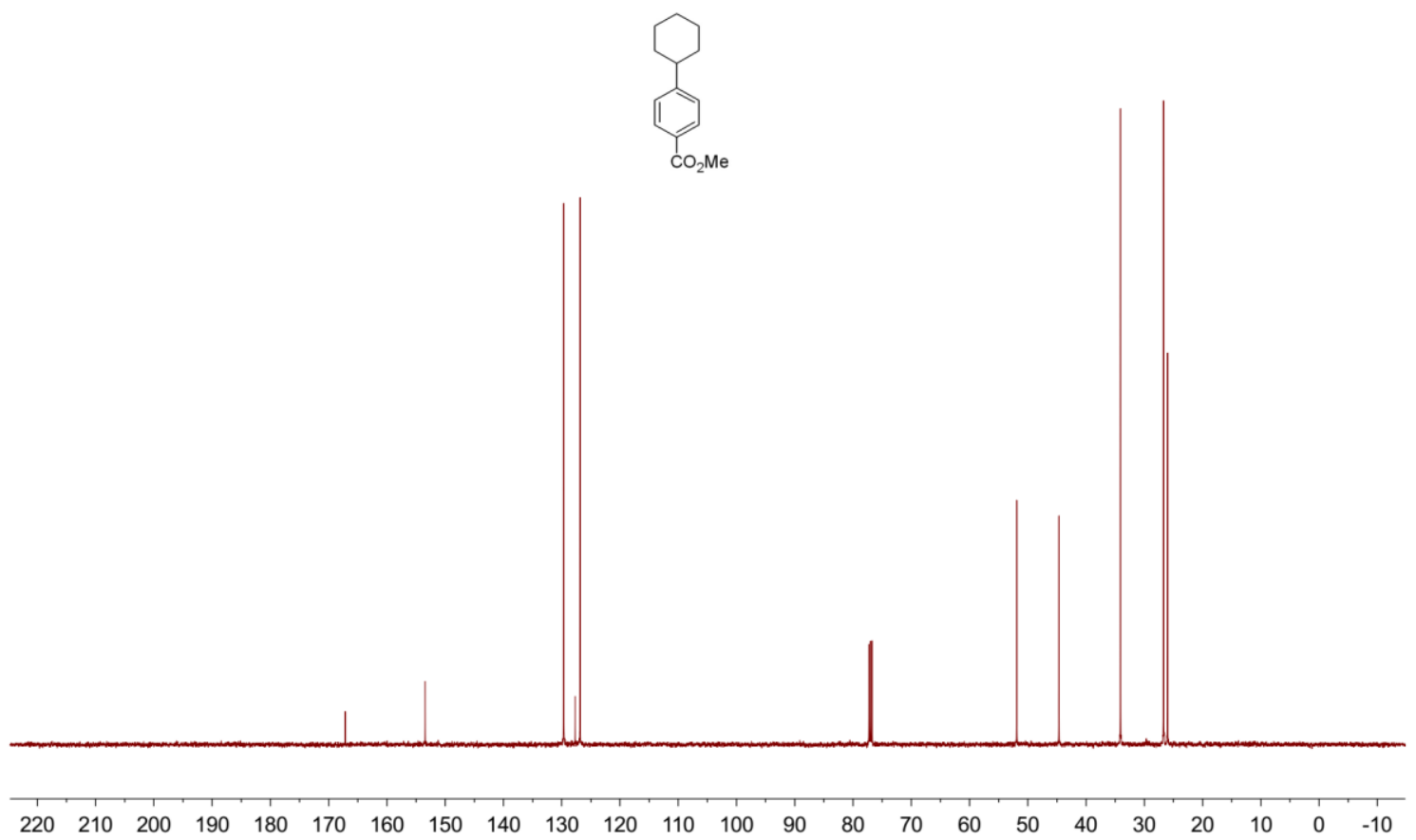


1H NMR (400 MHz,CDCł) for $\mathbf{4 p}$

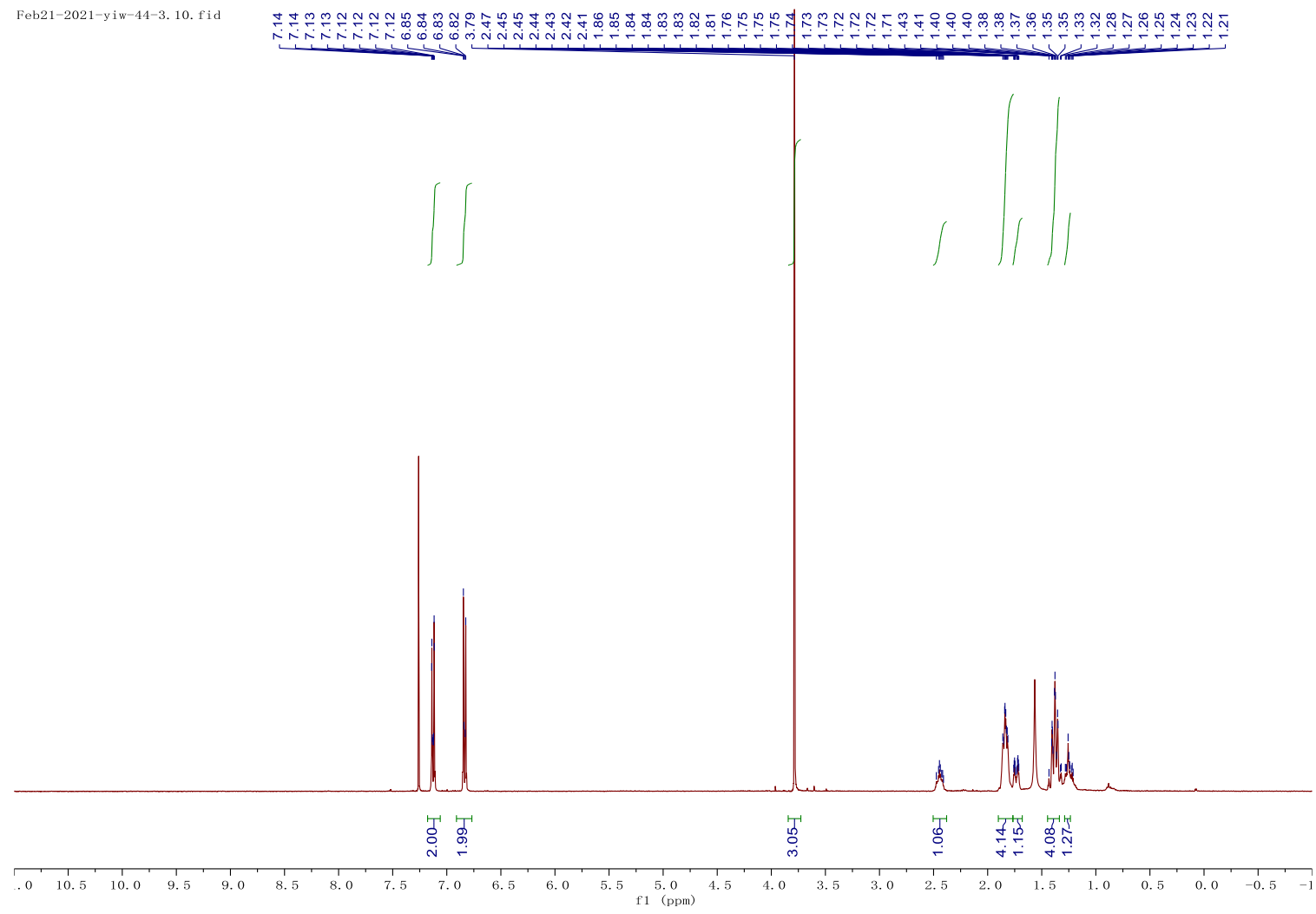

13C NMR (101 MHz,CDCł) for 4p
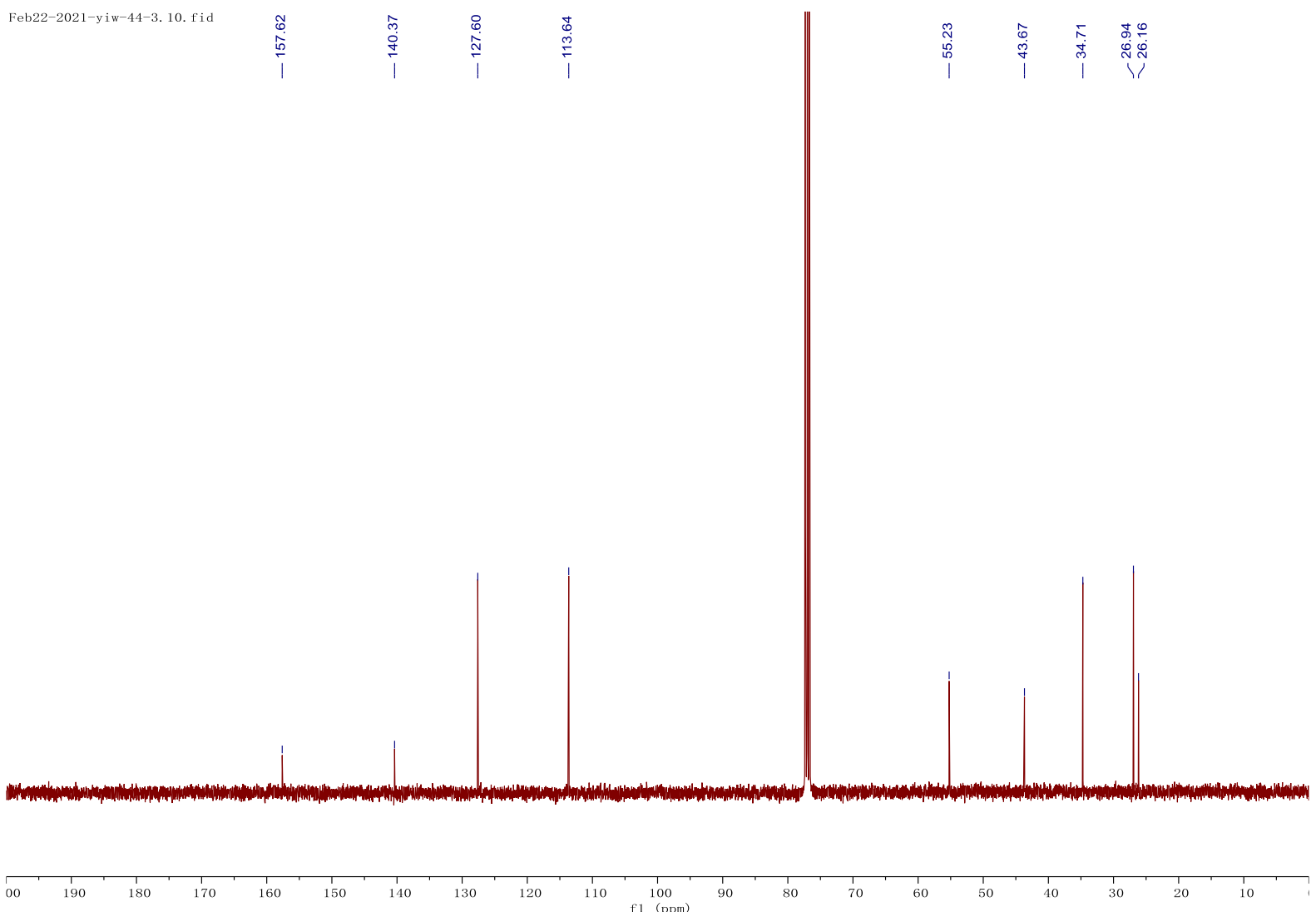
1H NMR (500 MHz, CDG) for 4q

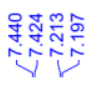

芯澹
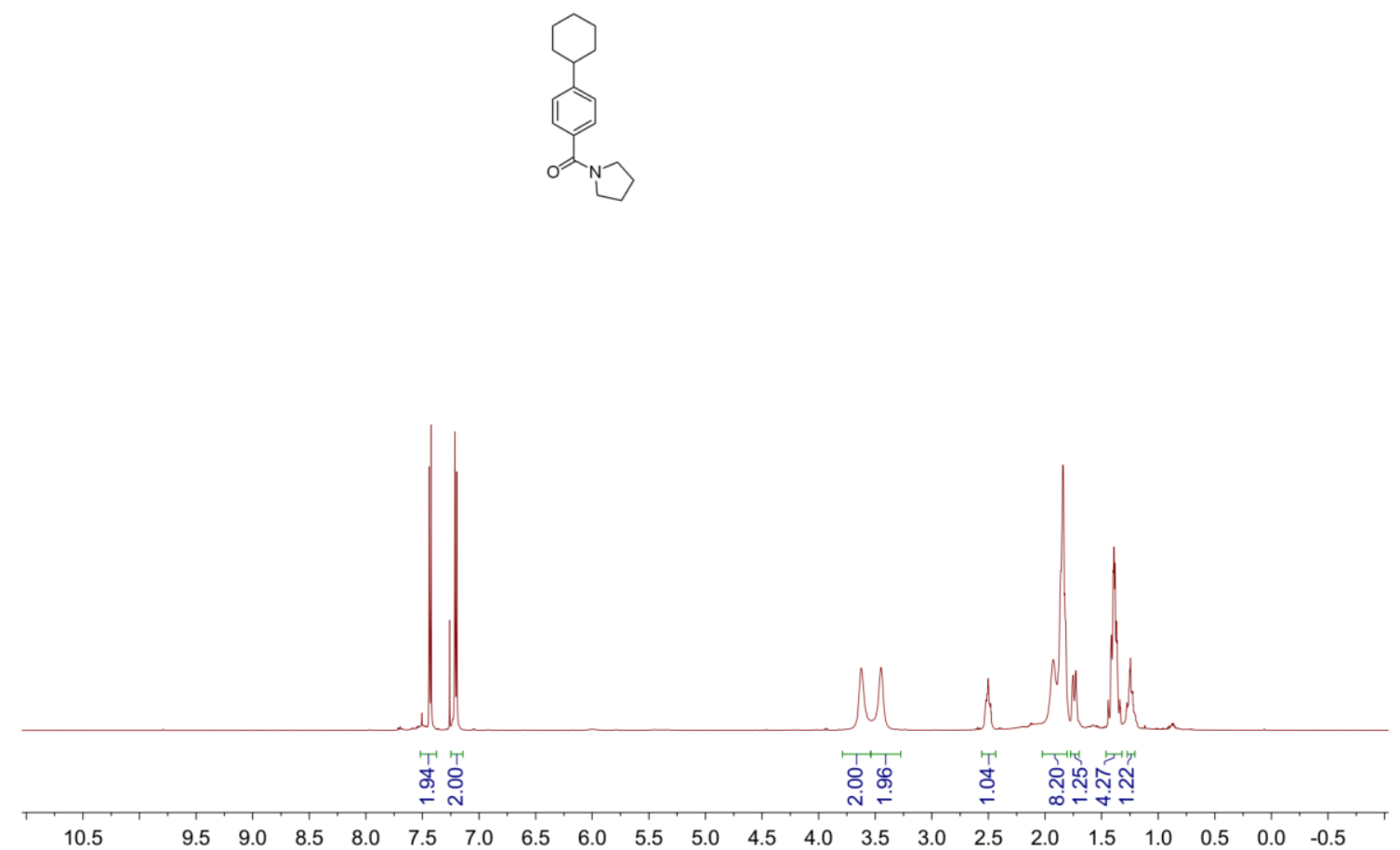

13C NMR (126 MHz, CDG) for $\mathbf{4 q}$

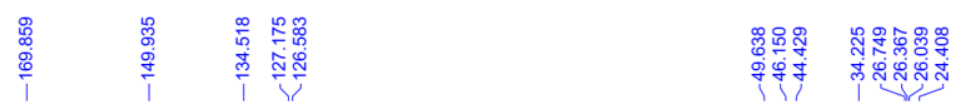

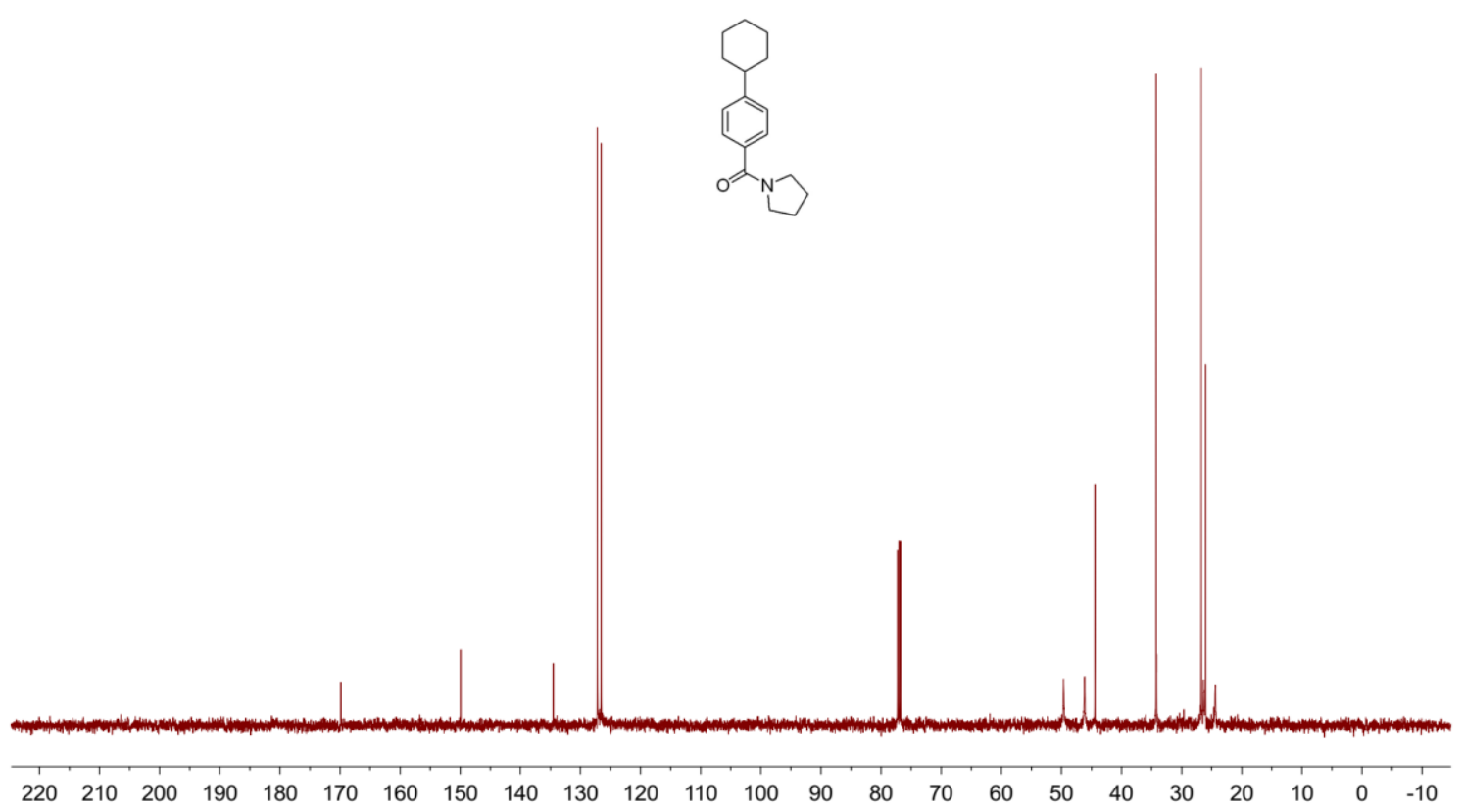


1H NMR (500 MHz, CDG) for $\mathbf{4 r}$

yiw44-1mn-H. 1. fid

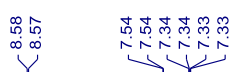

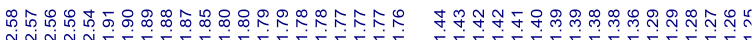

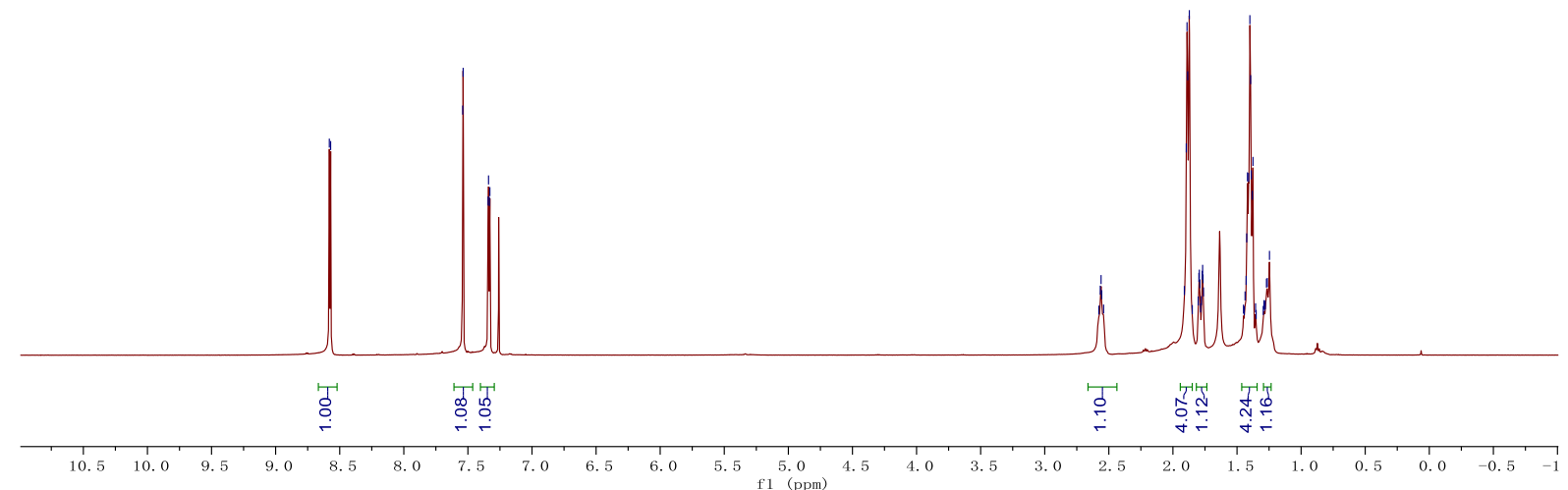

13C NMR (101 MHz, CDG) for 4r

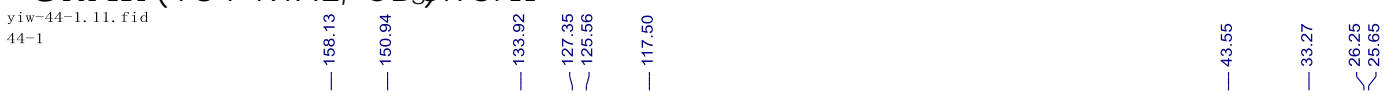
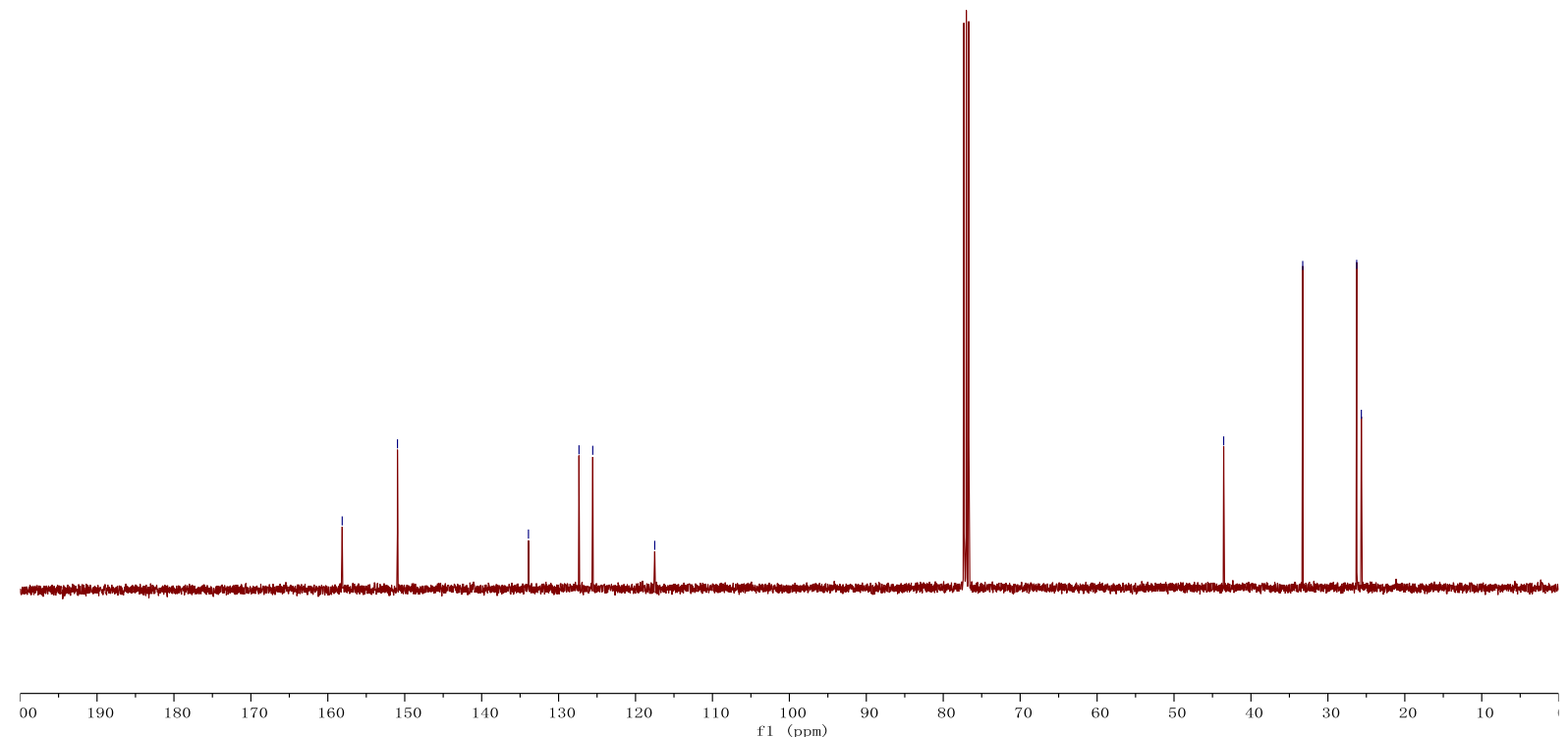
1H NMR (500 MHz, CDG) for 4s
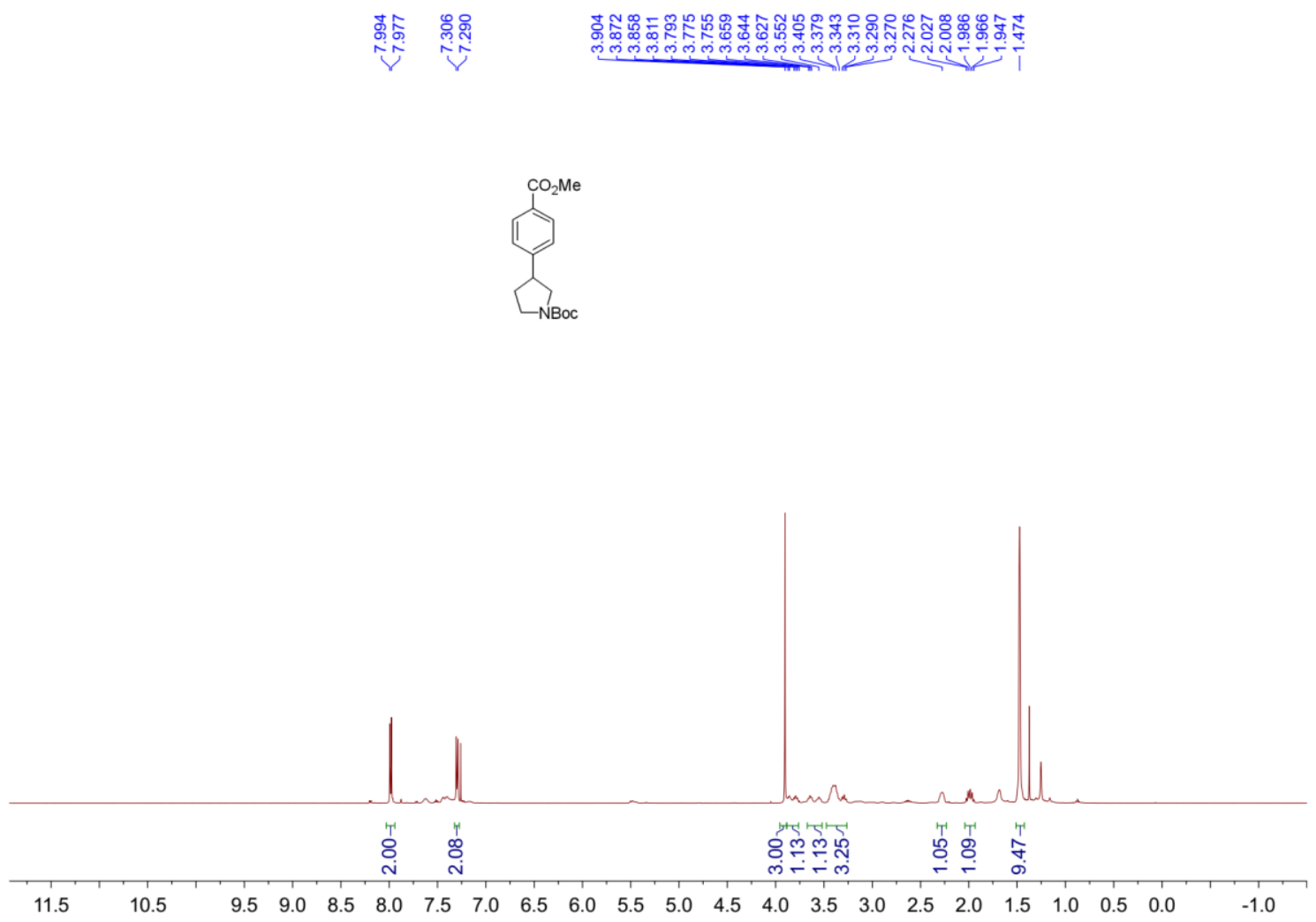

13C NMR (126 MHz, CDG) for $4 \mathbf{s}$

\begin{tabular}{|c|c|c|c|}
\hline 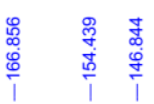 & 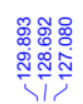 & 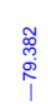 & 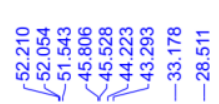 \\
\hline
\end{tabular}
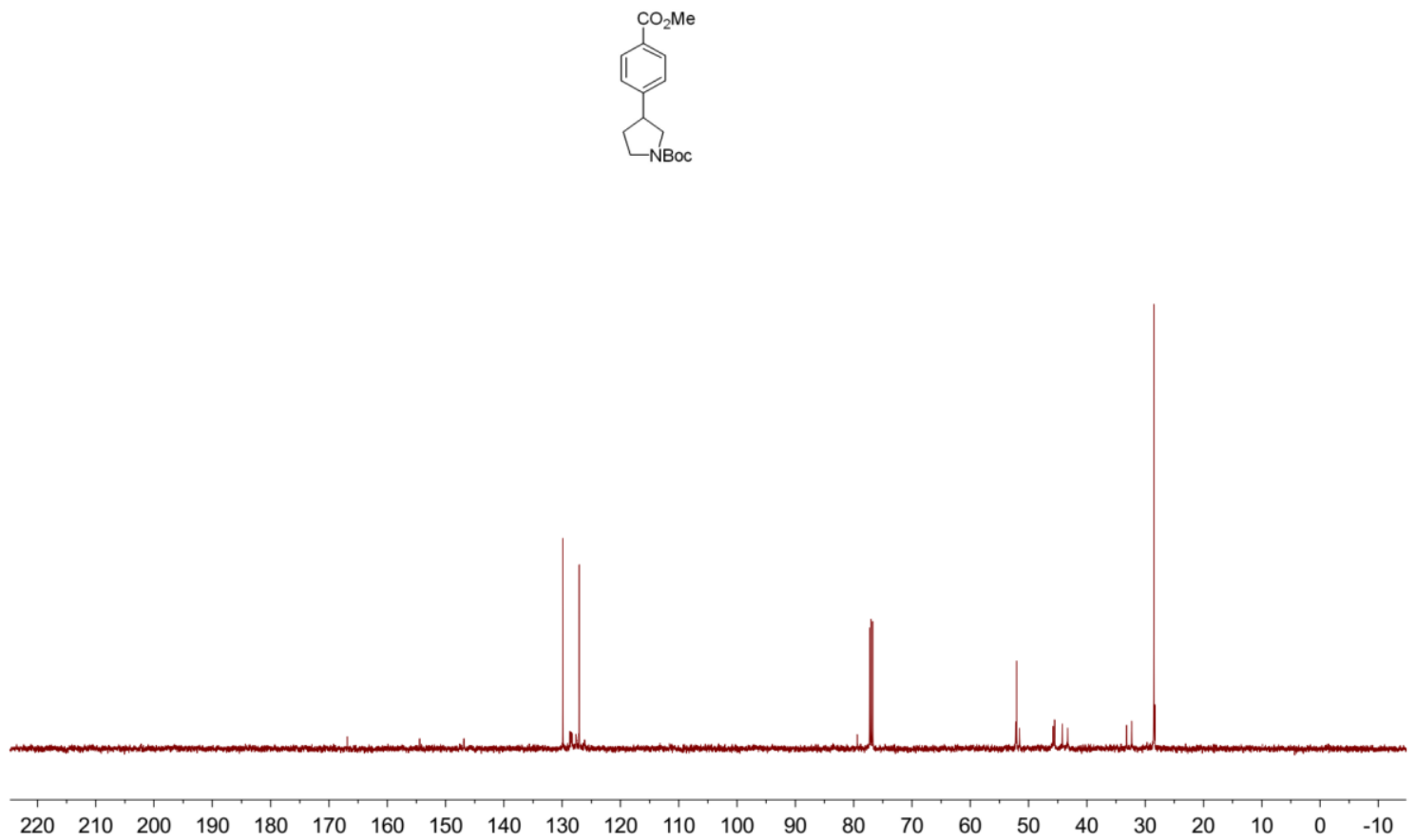
1H NMR (500 MHz, CDG) for $4 \mathbf{t}$

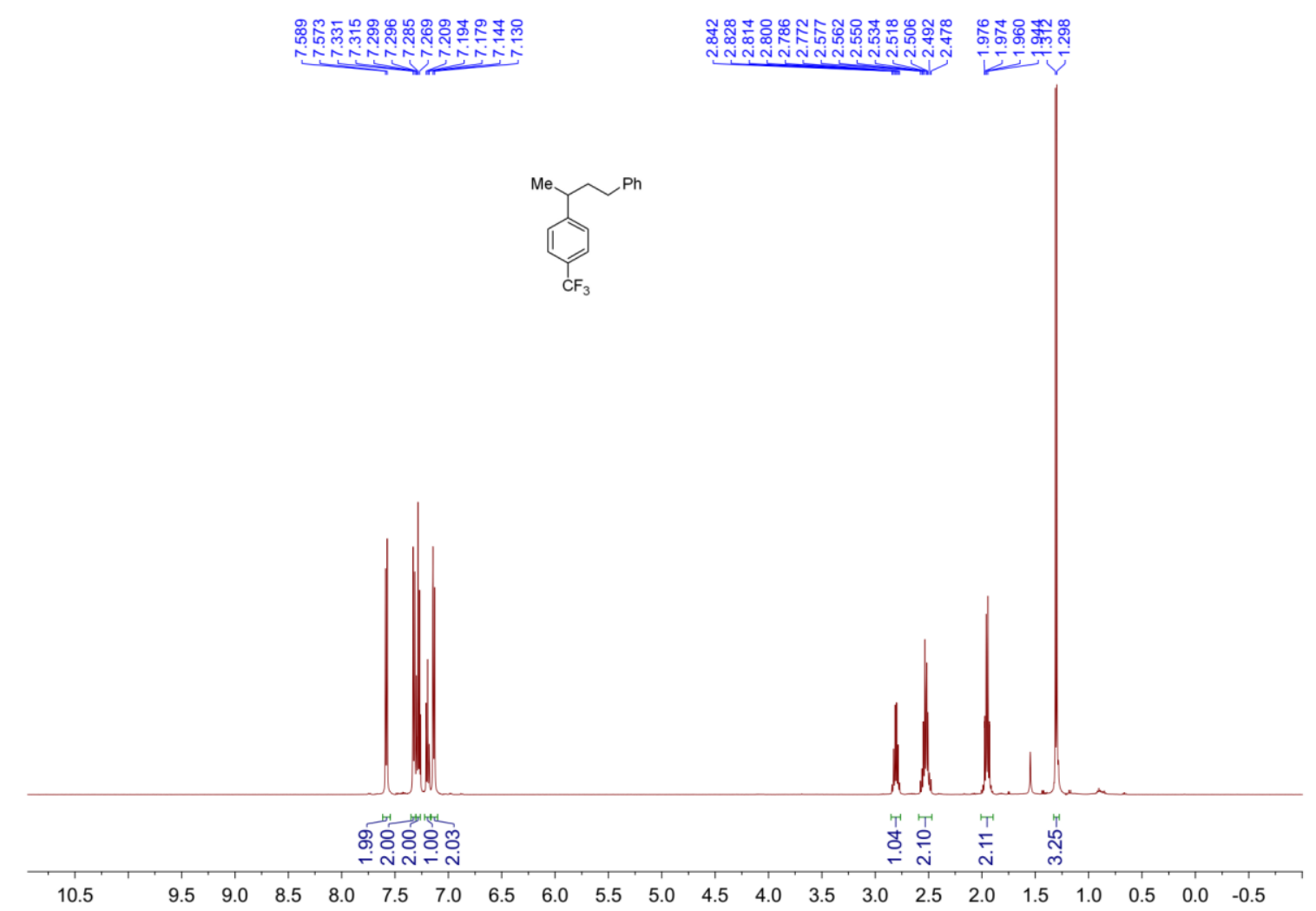

19F NMR (471 MHz, CDG) for $4 \mathbf{t}$

্ָণ<smiles>CC(CCCc1ccccc1)c1ccccc1</smiles>

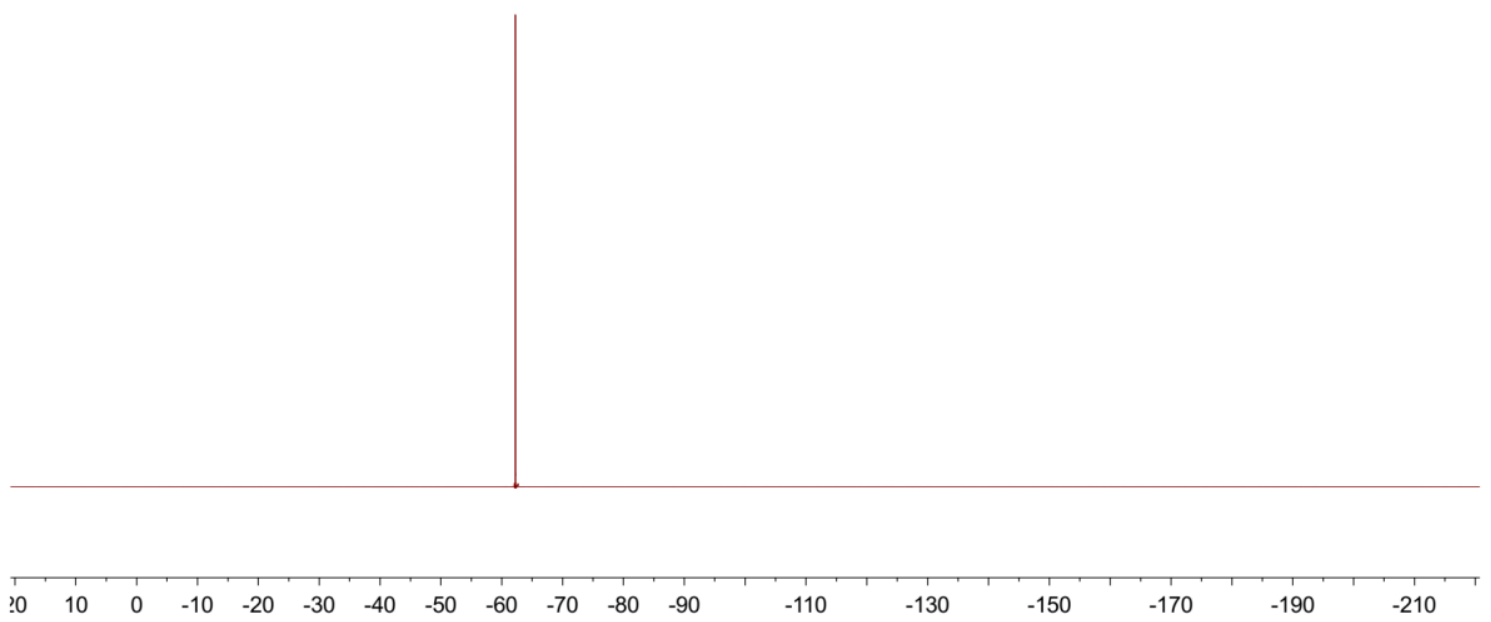


13C NMR (126 MHz, CDG) for $\mathbf{4 t}$

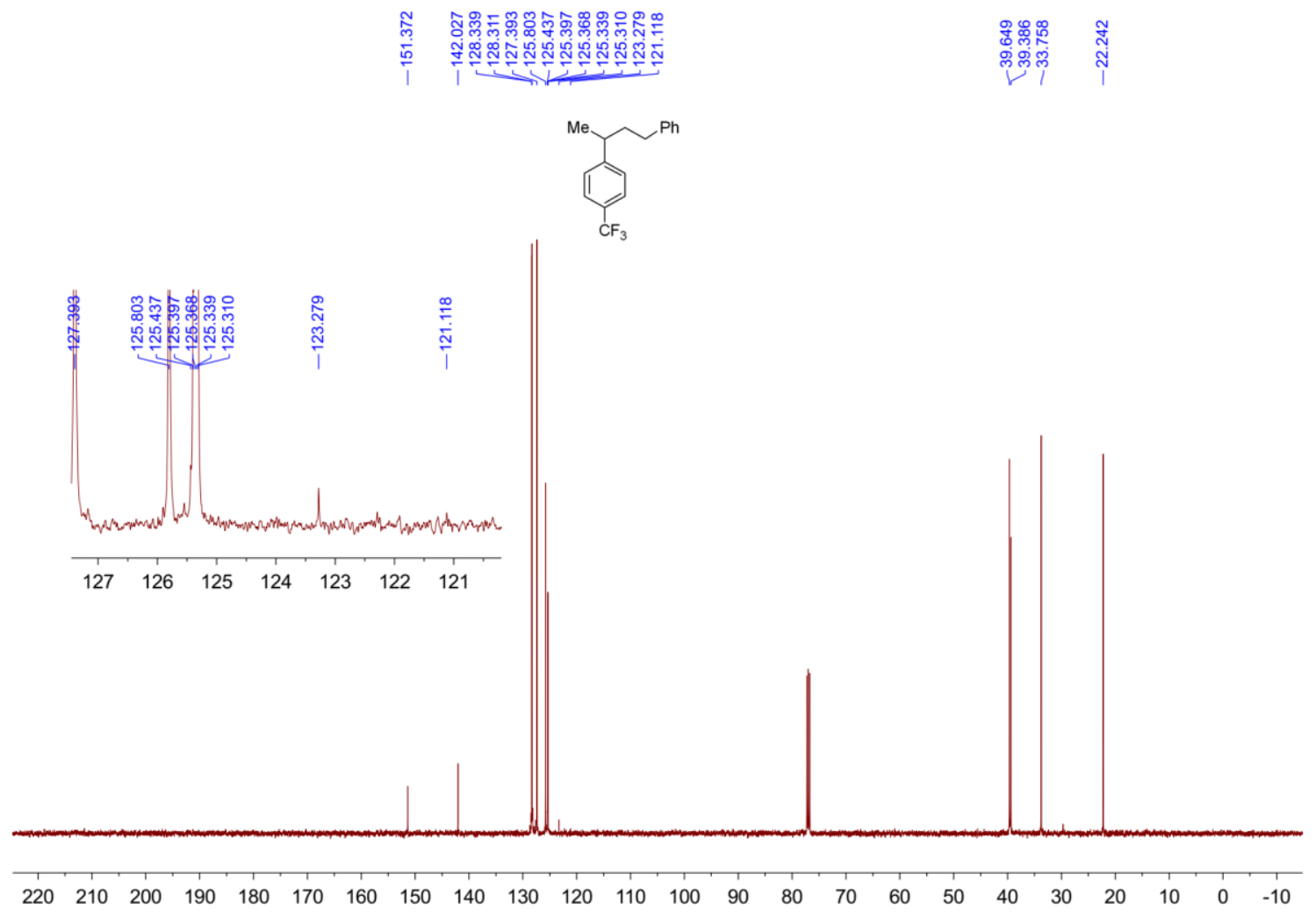


1H NMR (500 MHz, CDG) for $\mathbf{4 u}$

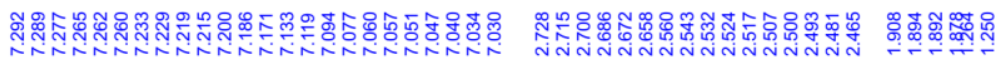

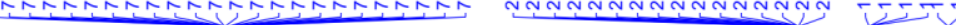
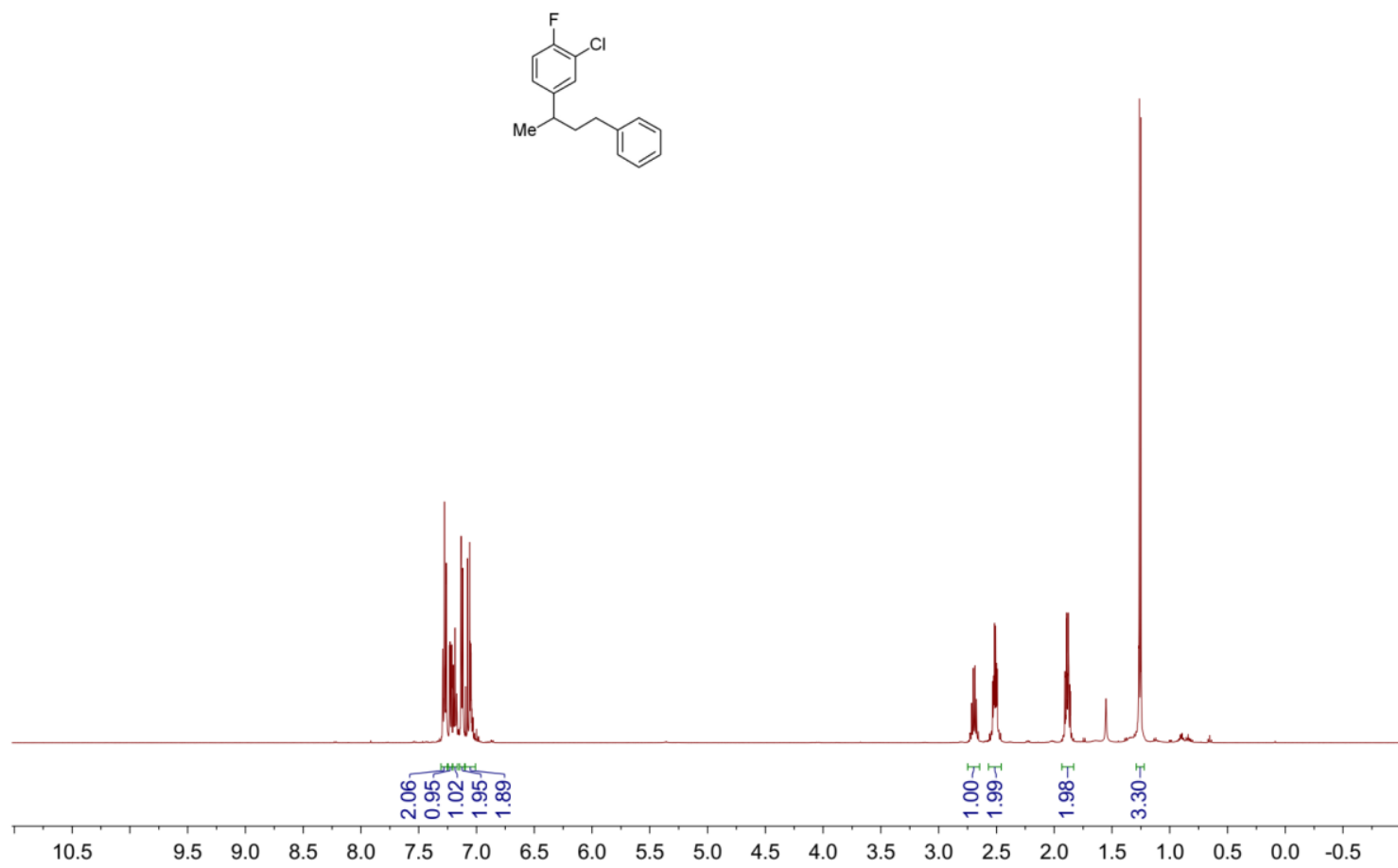

19F NMR (471 MHz, CDG) for $\mathbf{4 u}$
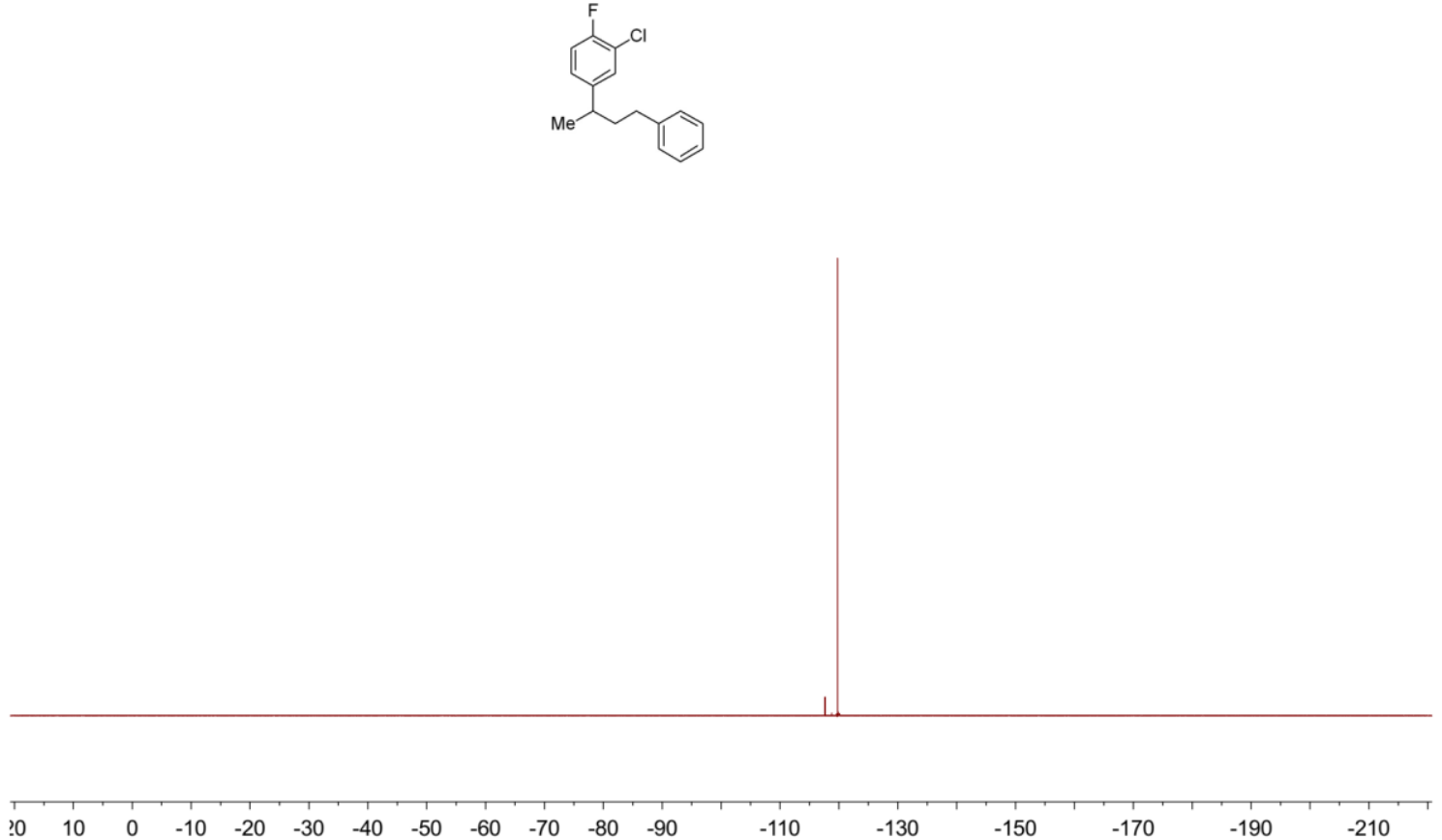
13C NMR (126 MHz, CDG) for $\mathbf{4 u}$

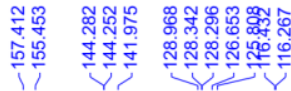

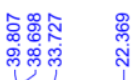
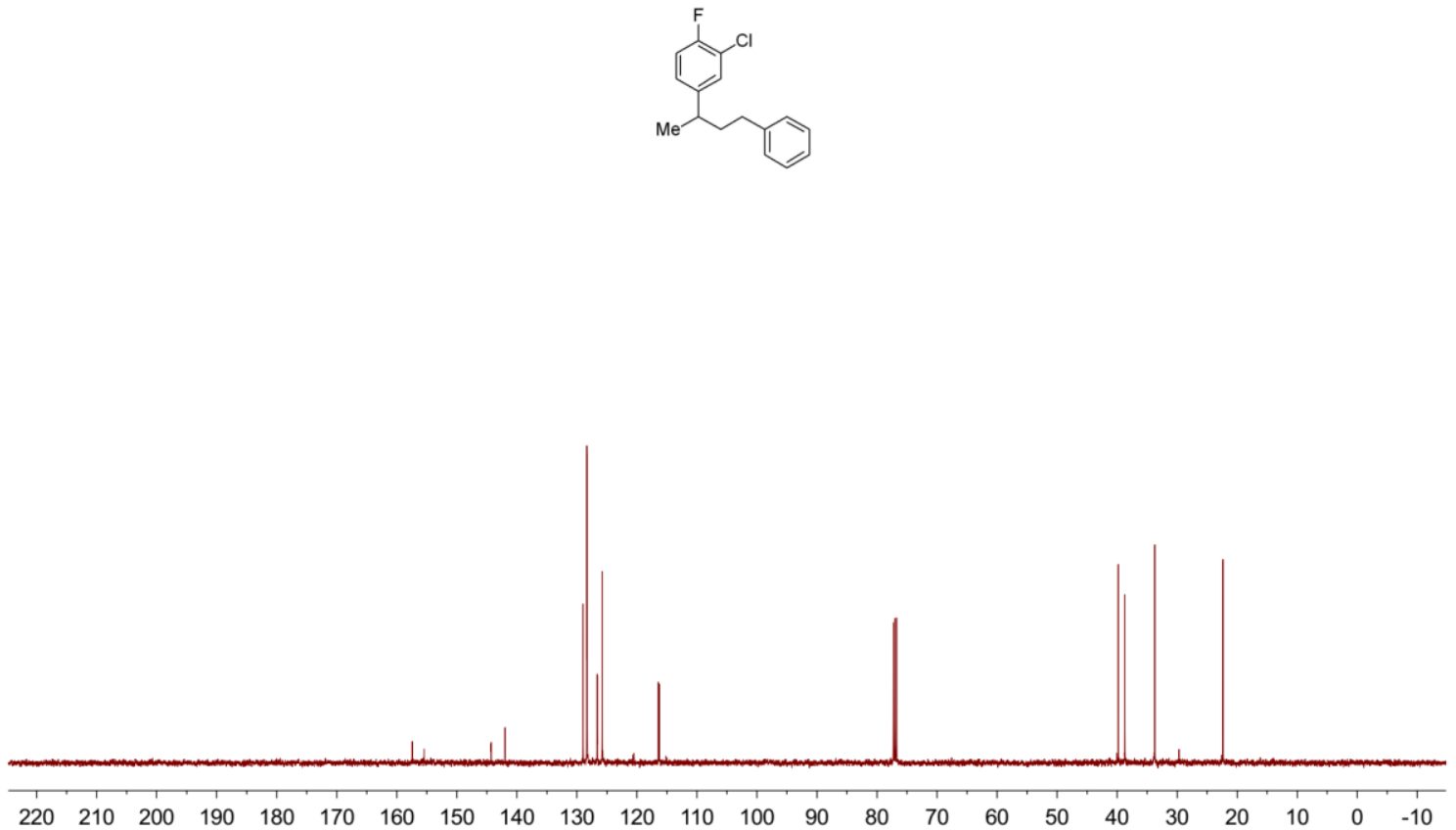
1H NMR (500 MHz, CDG) for $\mathbf{4 v}$

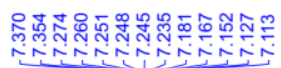

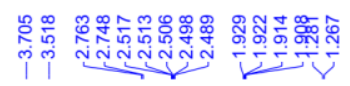
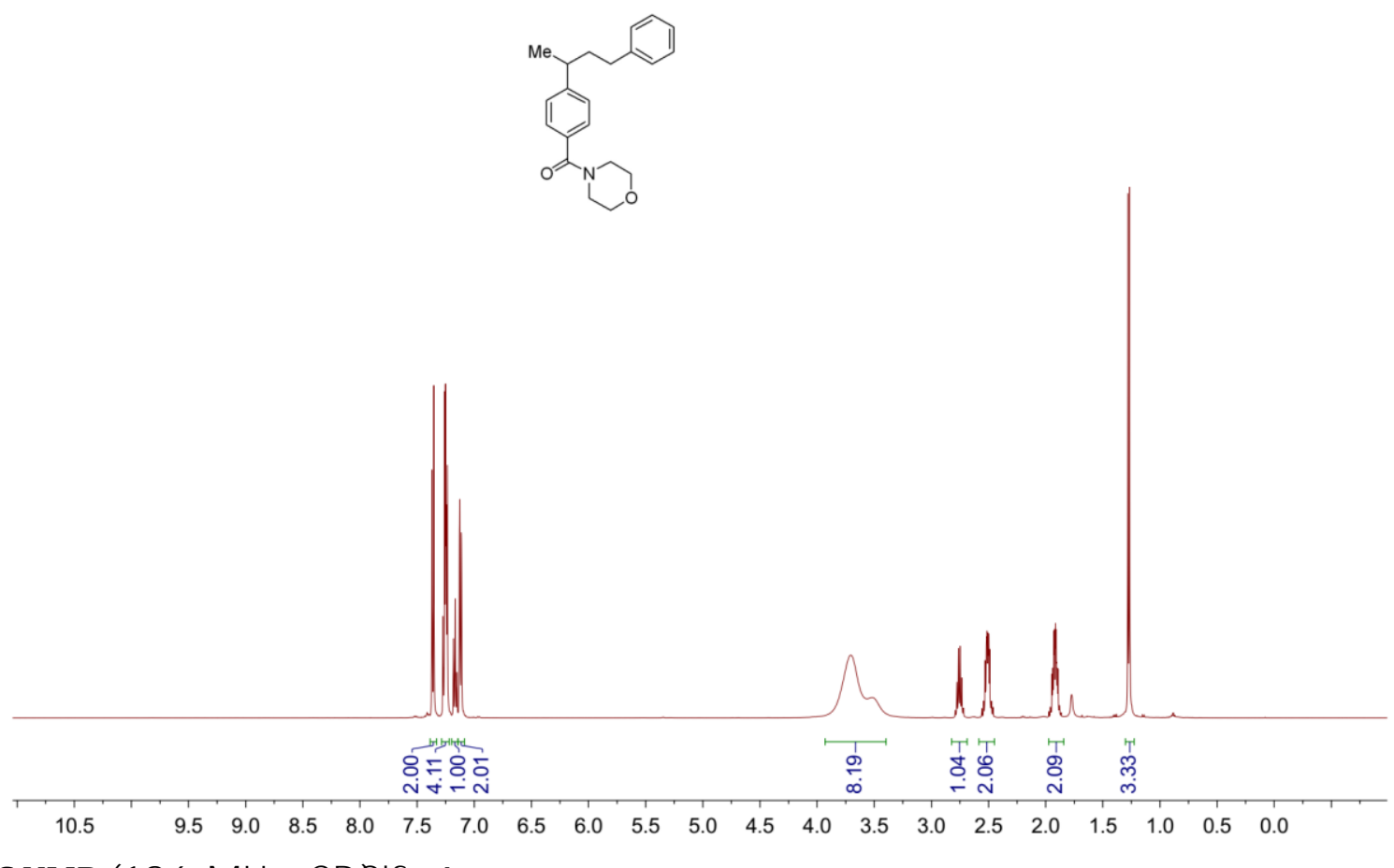

13C NMR (126 MHz, CDG) for $\mathbf{4 v}$

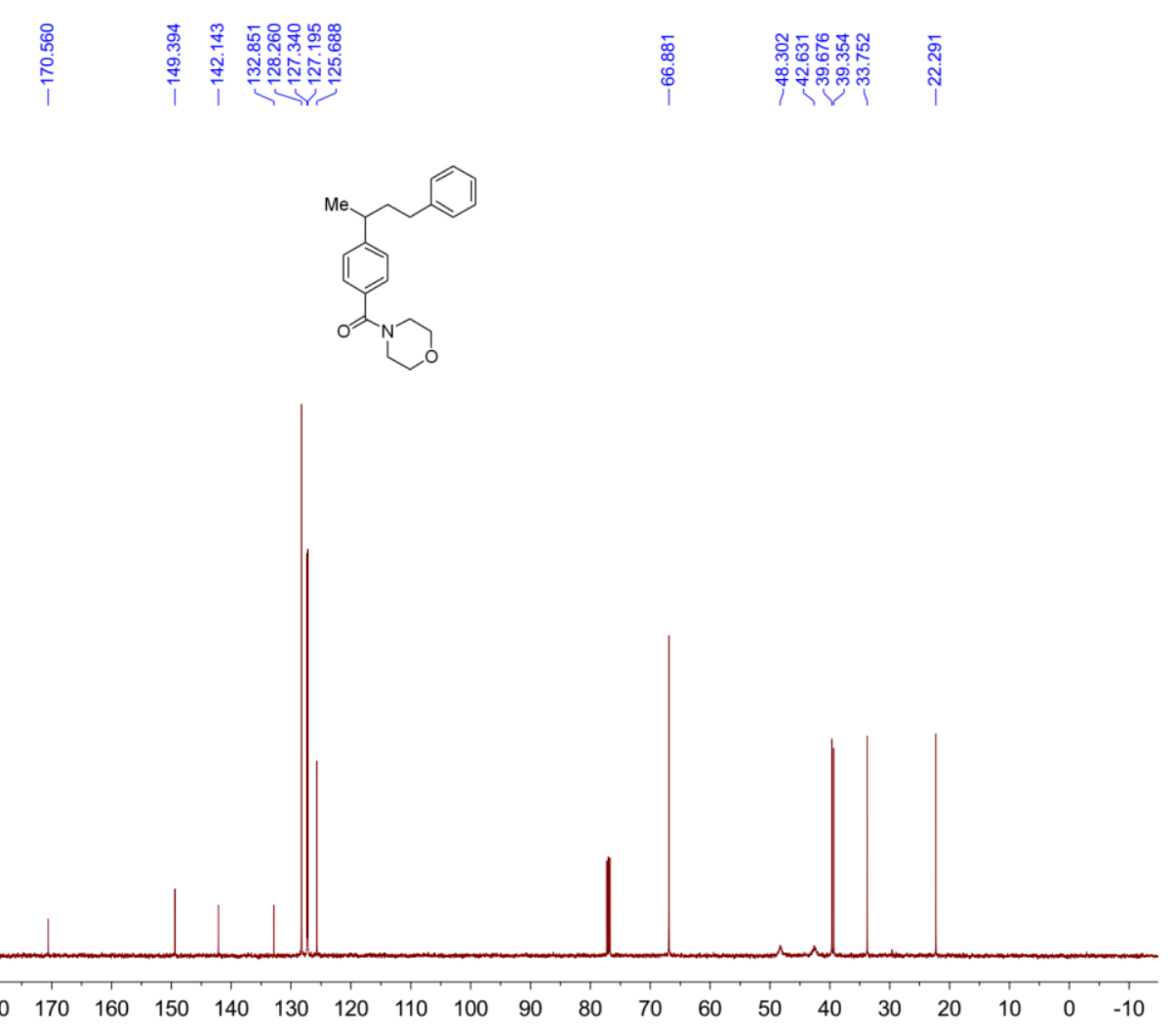

54 
1H NMR (500 MHz, CDG) for $\mathbf{4 w}$

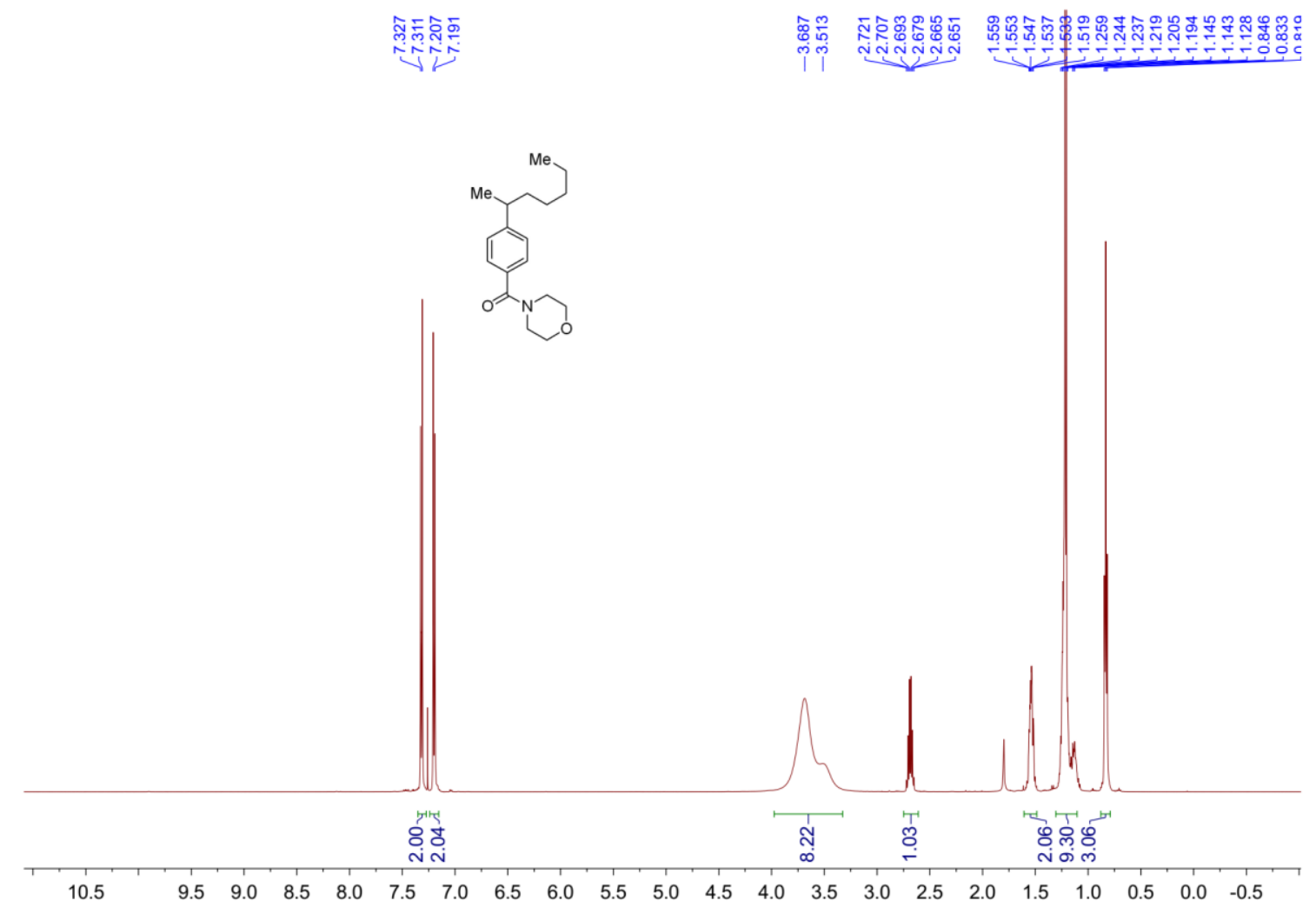

13C NMR (126 MHz, CDG) for $\mathbf{4} \mathbf{w}$

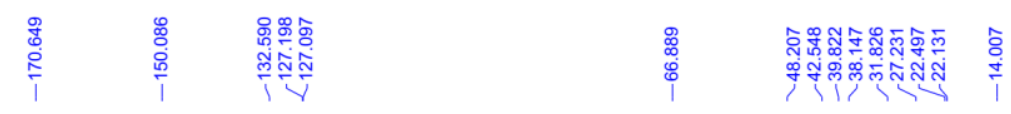

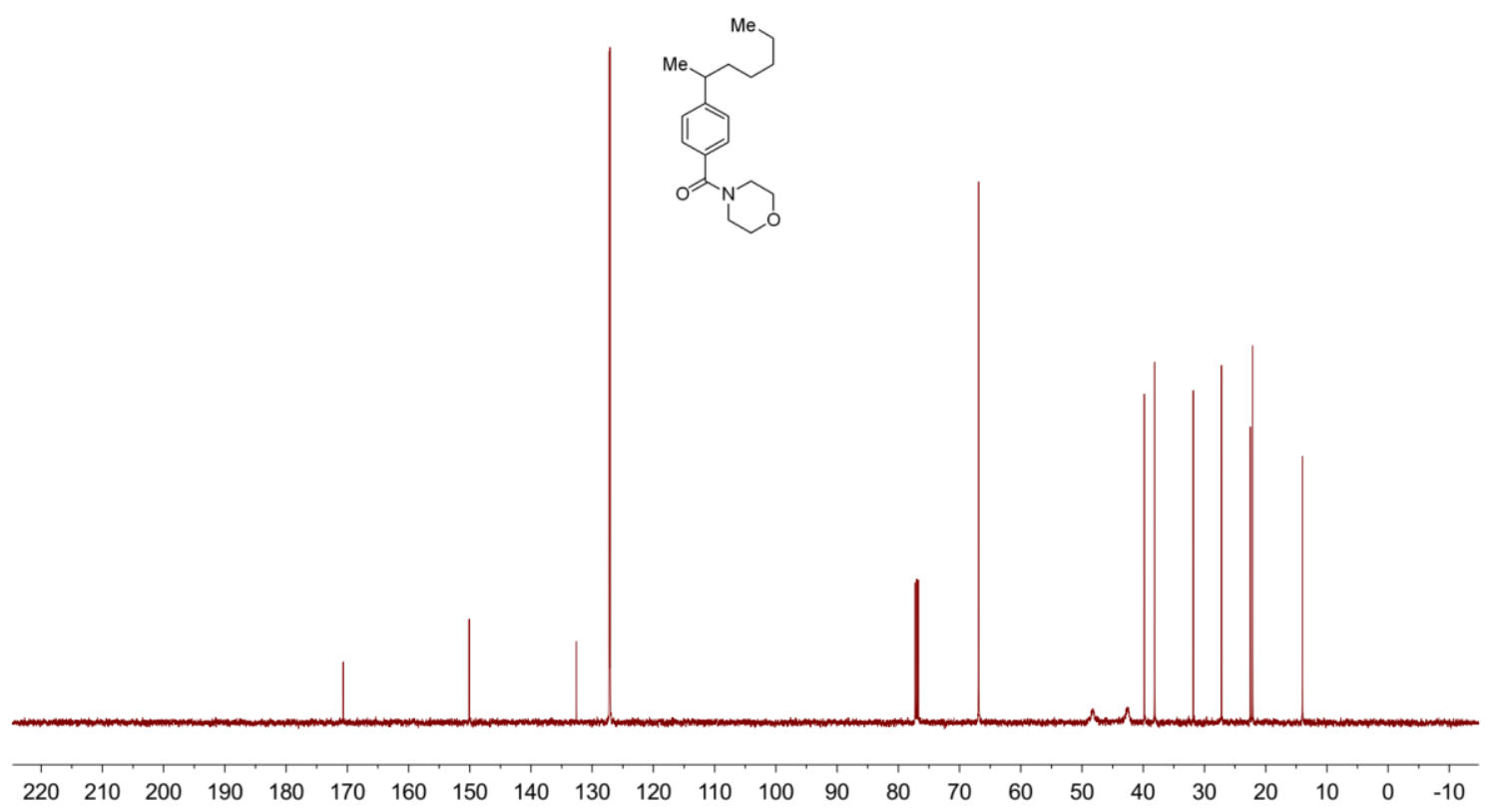


1H NMR (500 MHz, CDG) for $\mathbf{4 x}$
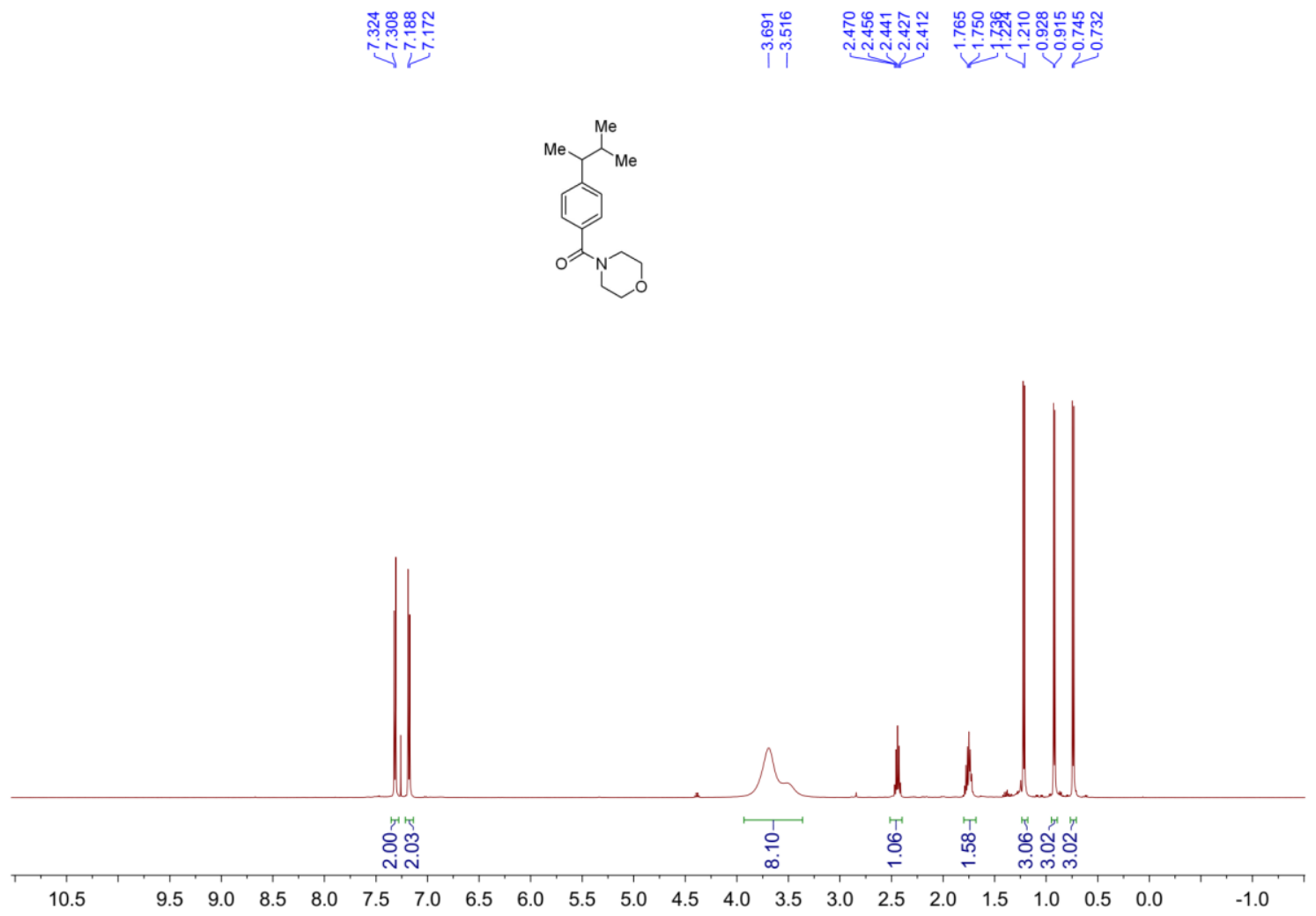

13C NMR (126 MHz, CDG) for $\mathbf{4 x}$

\begin{tabular}{|c|c|c|c|}
\hline 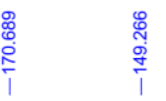 & 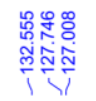 & $\begin{array}{l}0 \\
0 \\
0 \\
0 \\
1\end{array}$ & 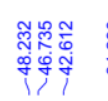 \\
\hline
\end{tabular}<smiles>CC(C)C(C)c1ccc(C(=O)N2CCOCC2)cc1</smiles>

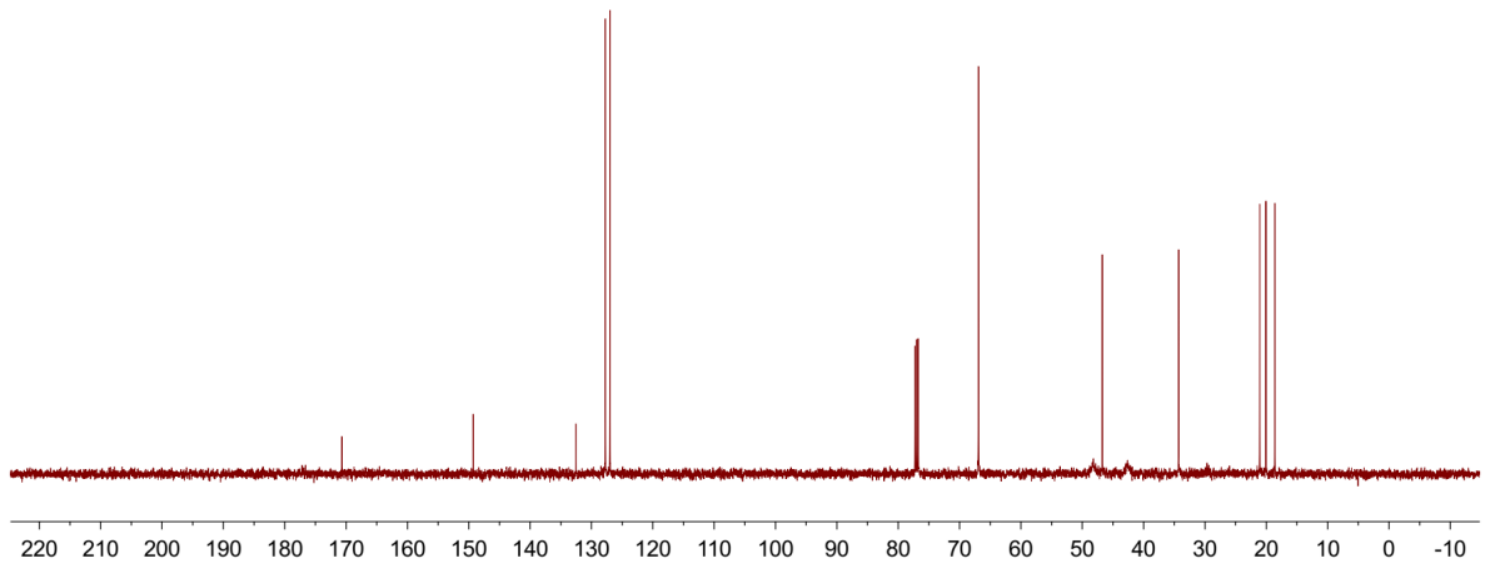


1H NMR (500 MHz, CDG) for $\mathbf{4 y}$
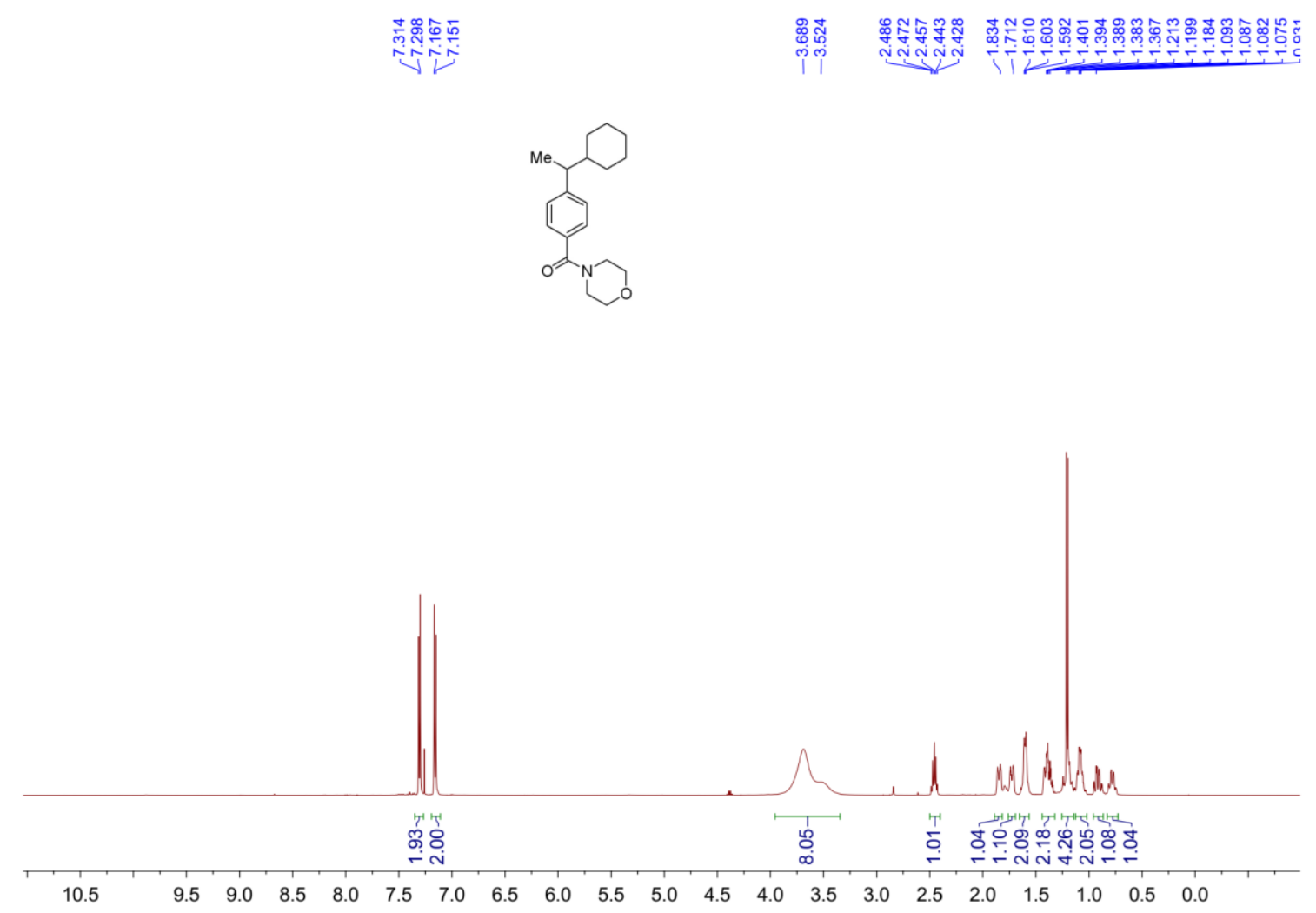

13C NMR (126 MHz, CDG) for $\mathbf{4 y}$

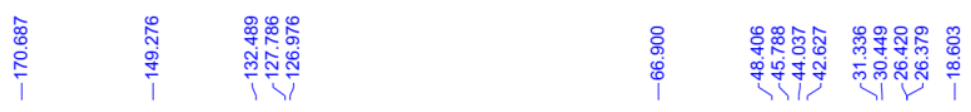

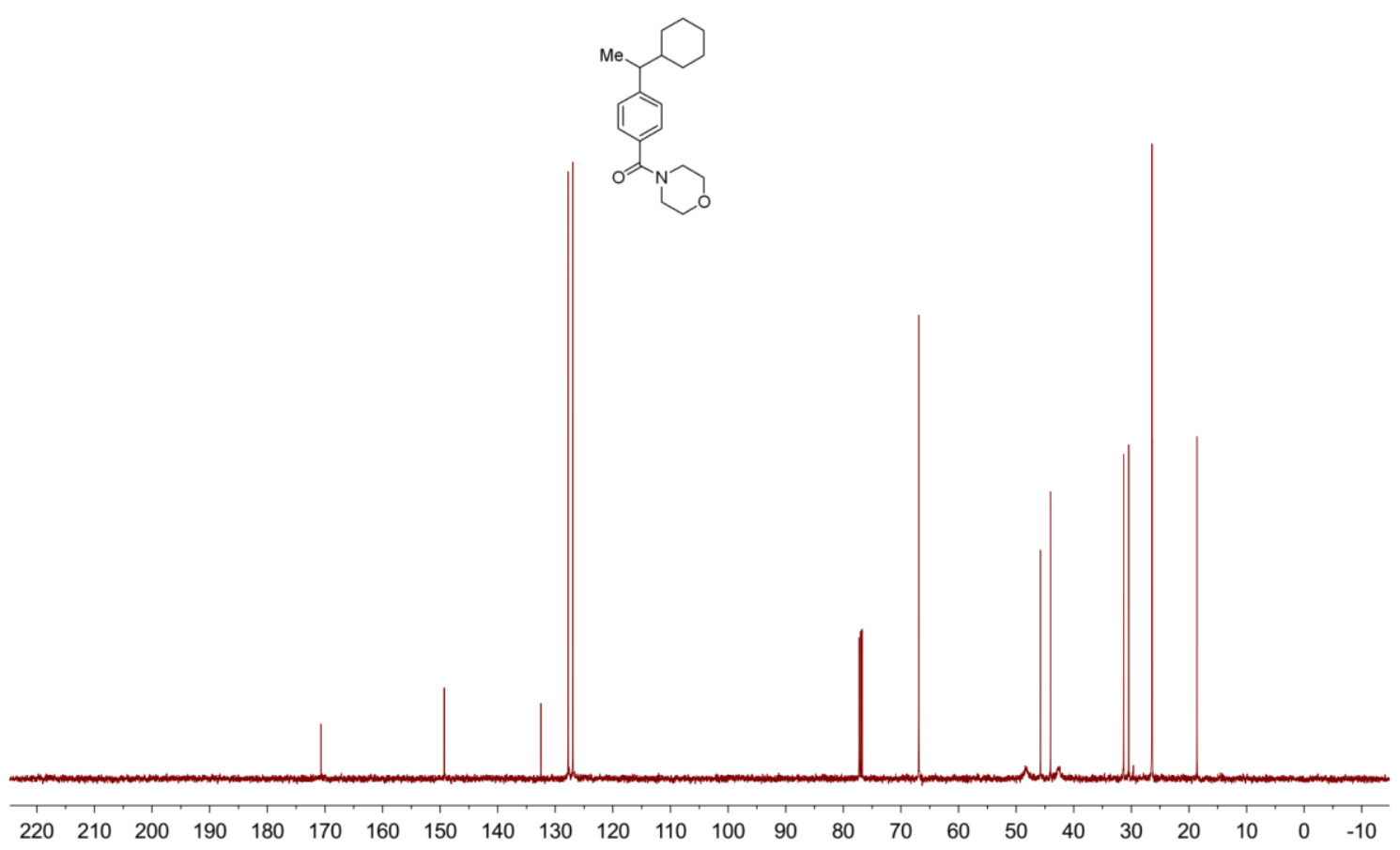


1H NMR (500 MHz, CDG) for $\mathbf{4 z}$

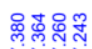

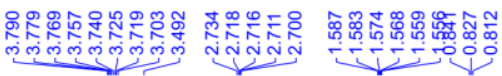

Nîn

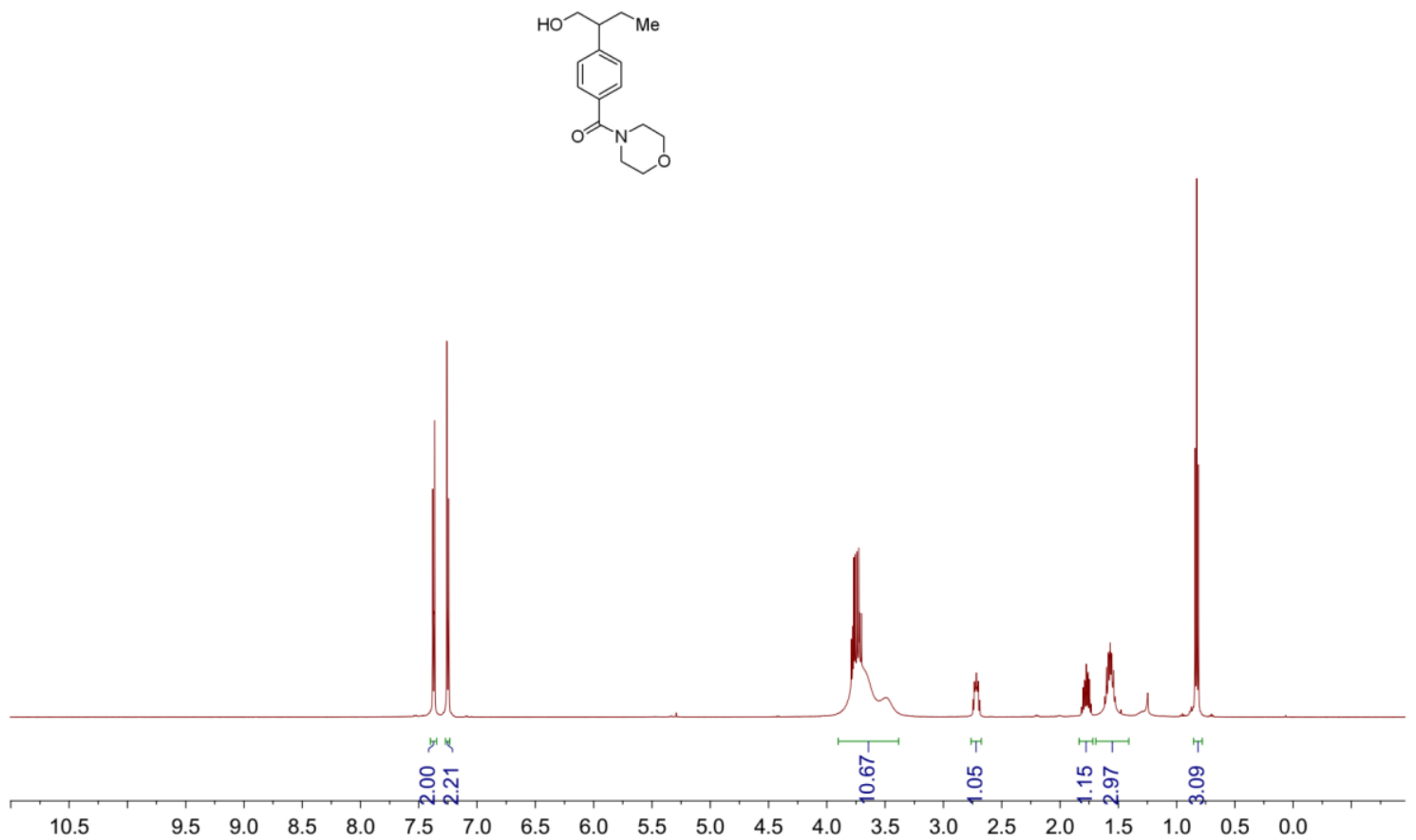

13C NMR (126 MHz, CDG) for $\mathbf{4 z}$
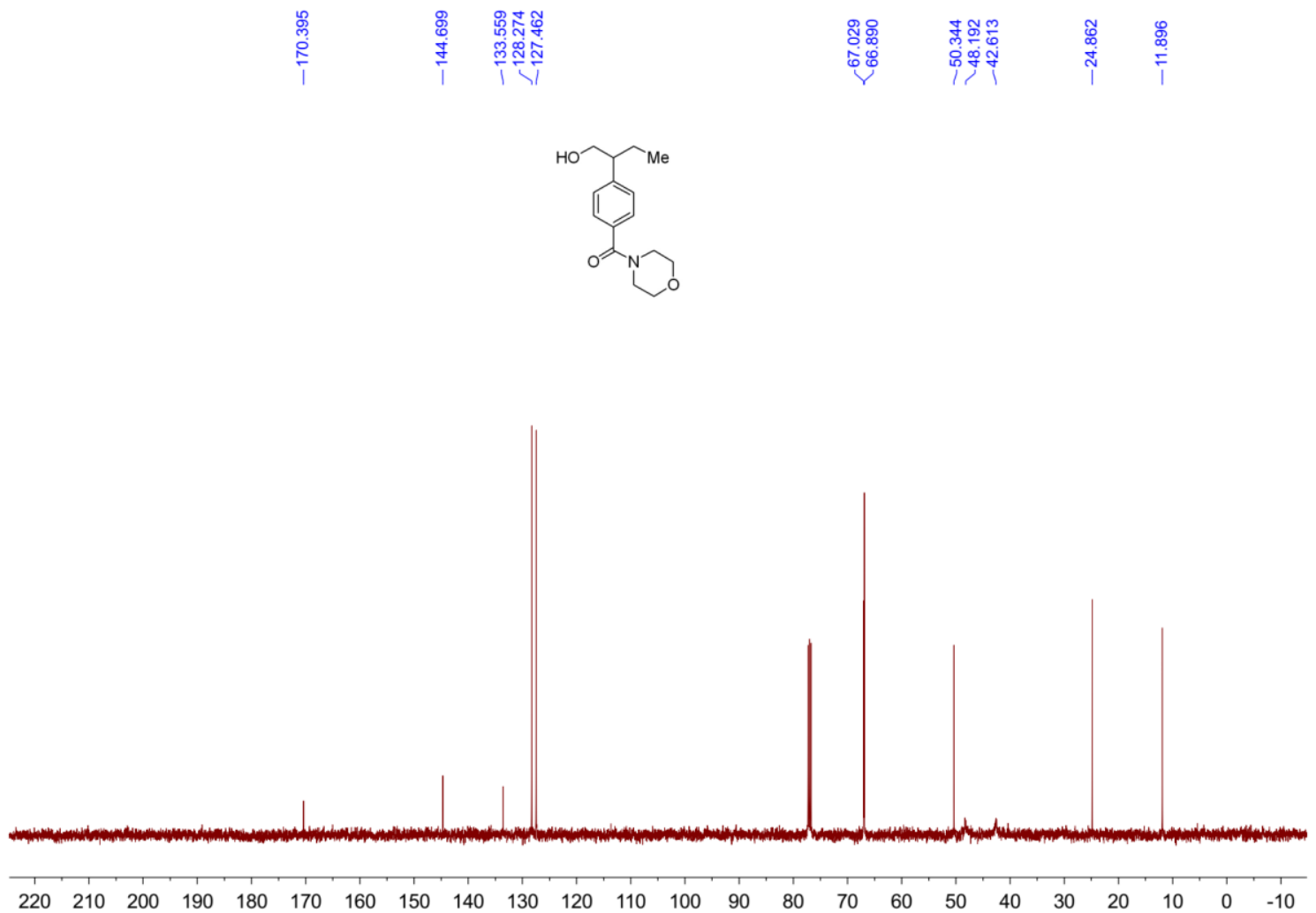
1H NMR (500 MHz, CDG) for 4aa

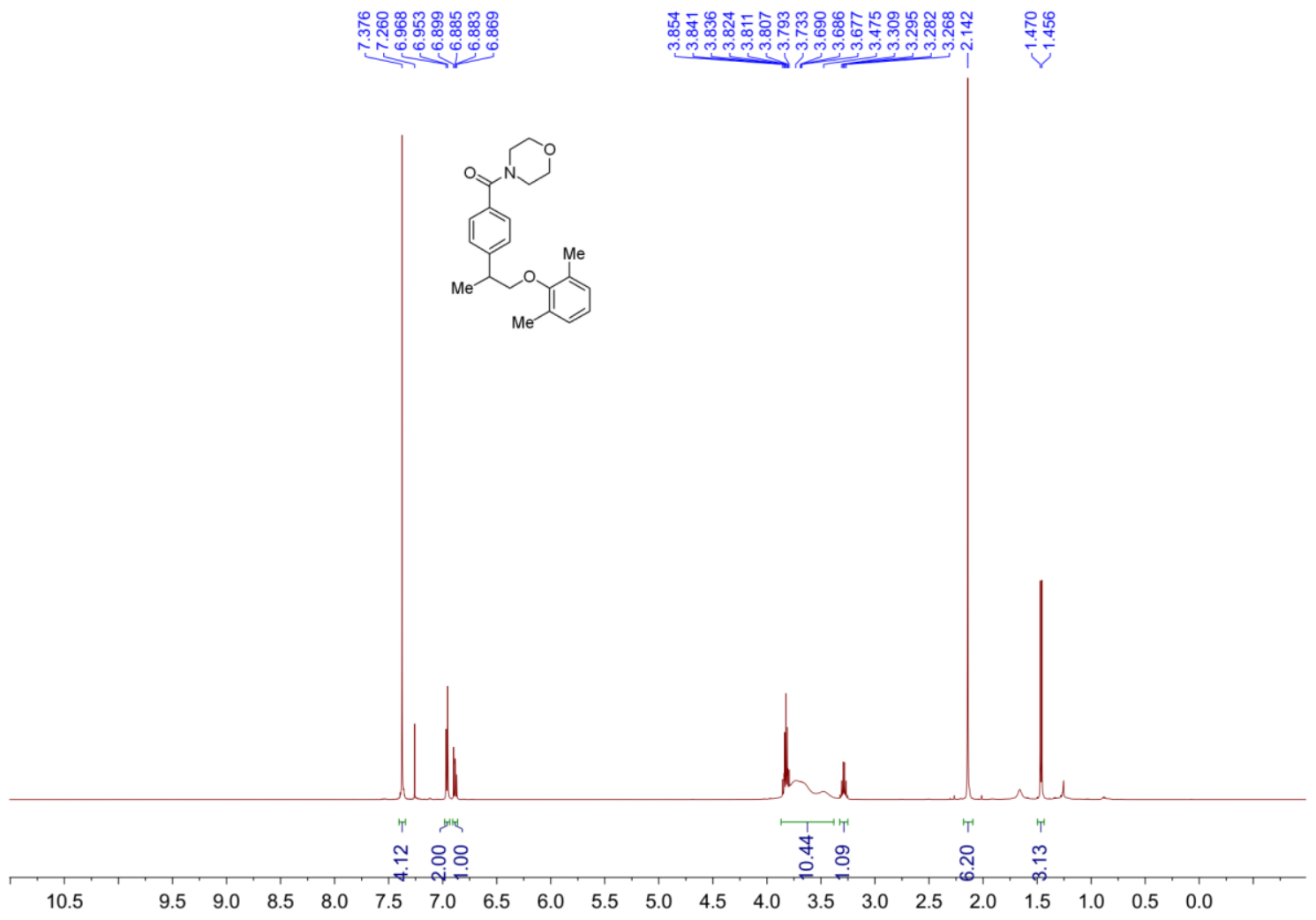

13C NMR (126 MHz, CDG) for 4aa

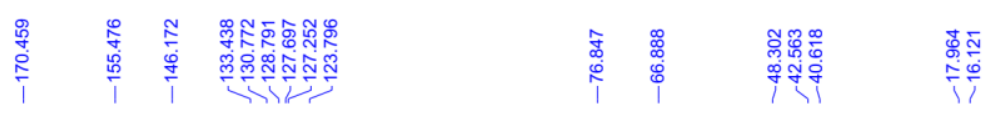

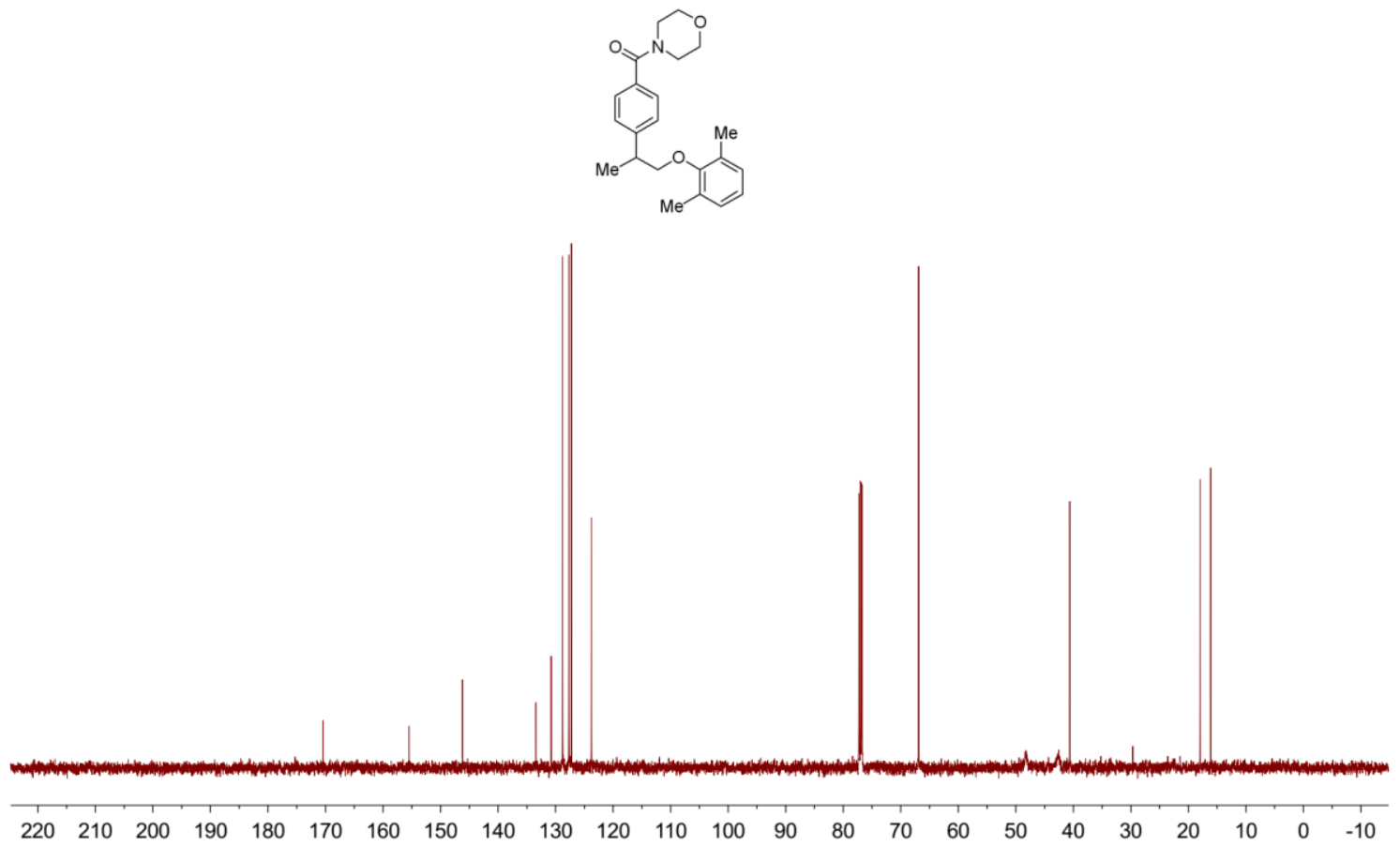


1H NMR (500 MHz, CDG) for $4 \mathbf{a b}$

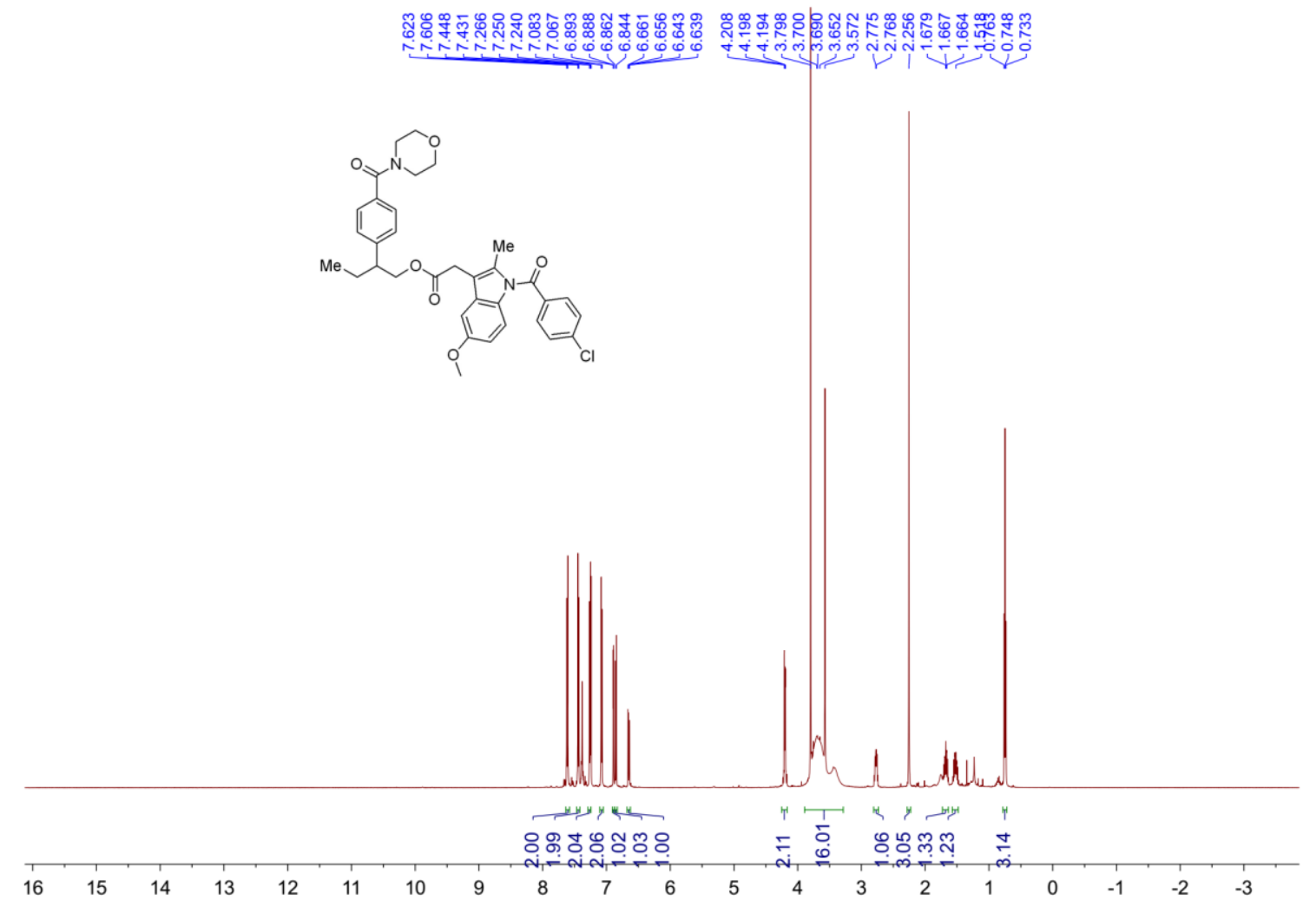

13C NMR (126 MHz, CDG) for 4ab
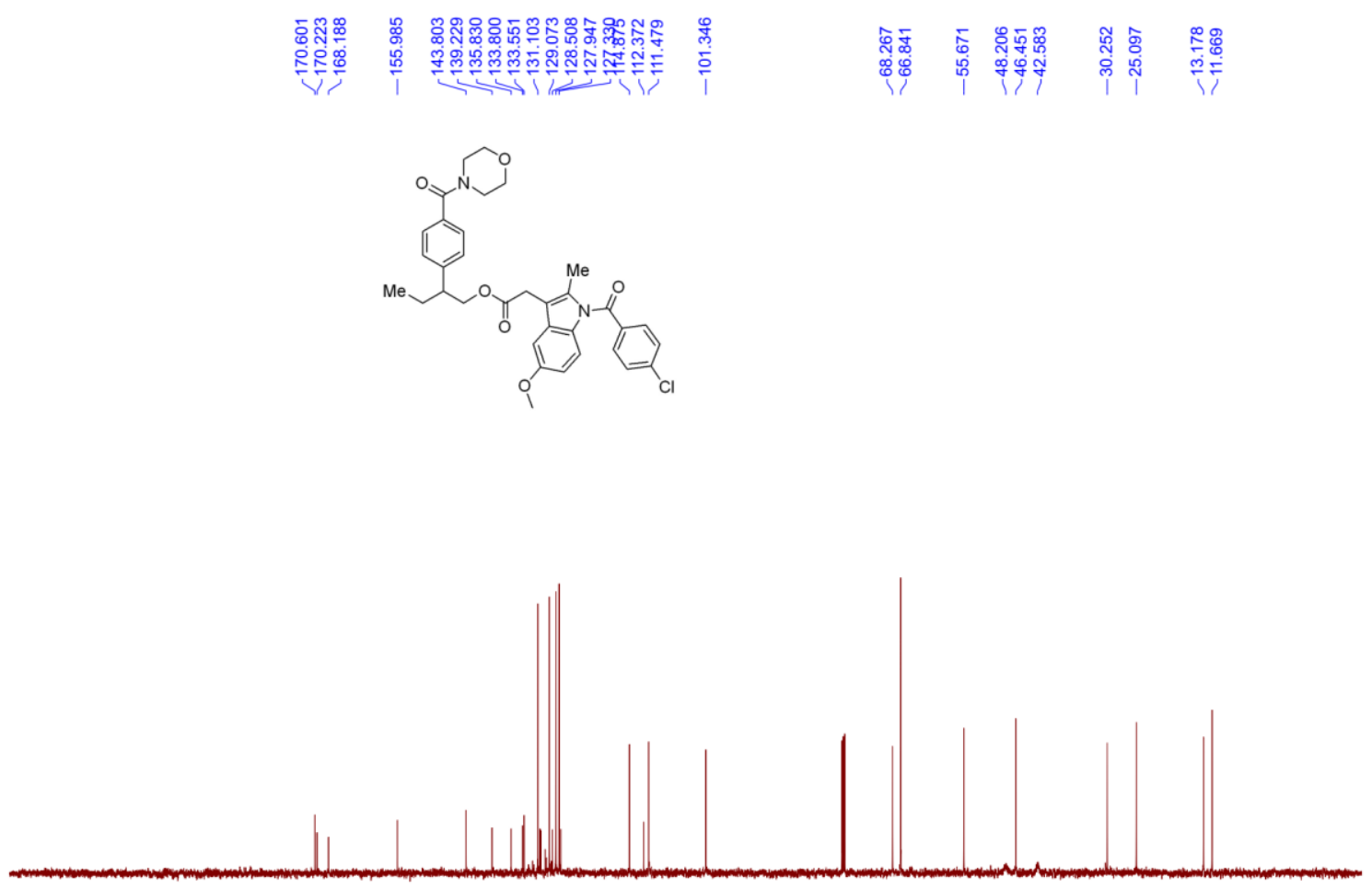

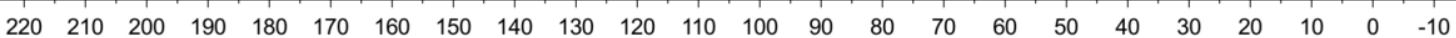


1H NMR (400 MHz, CDG) for 4 ac

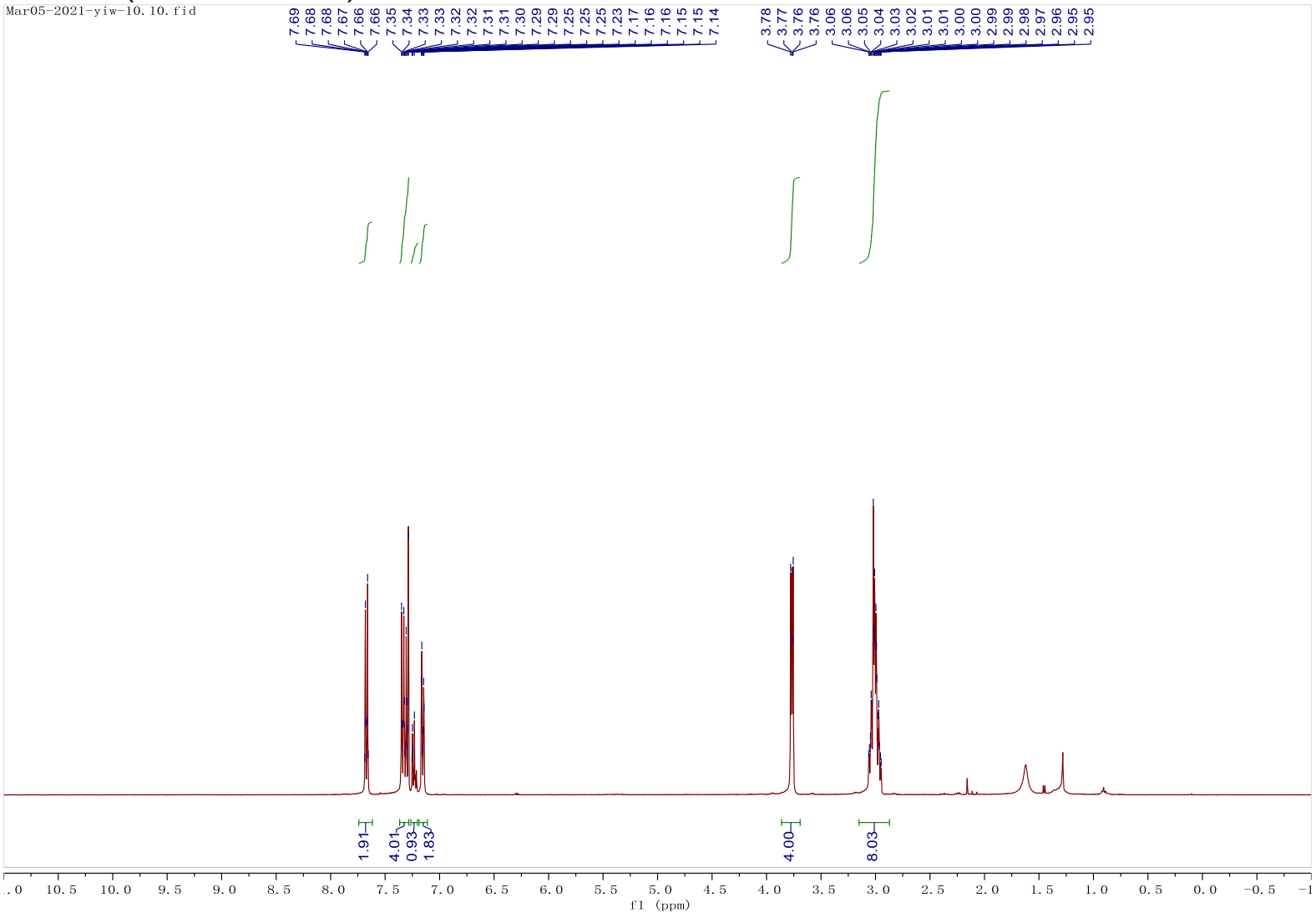

13C NMR (101 MHz, CDG) for 4 ac
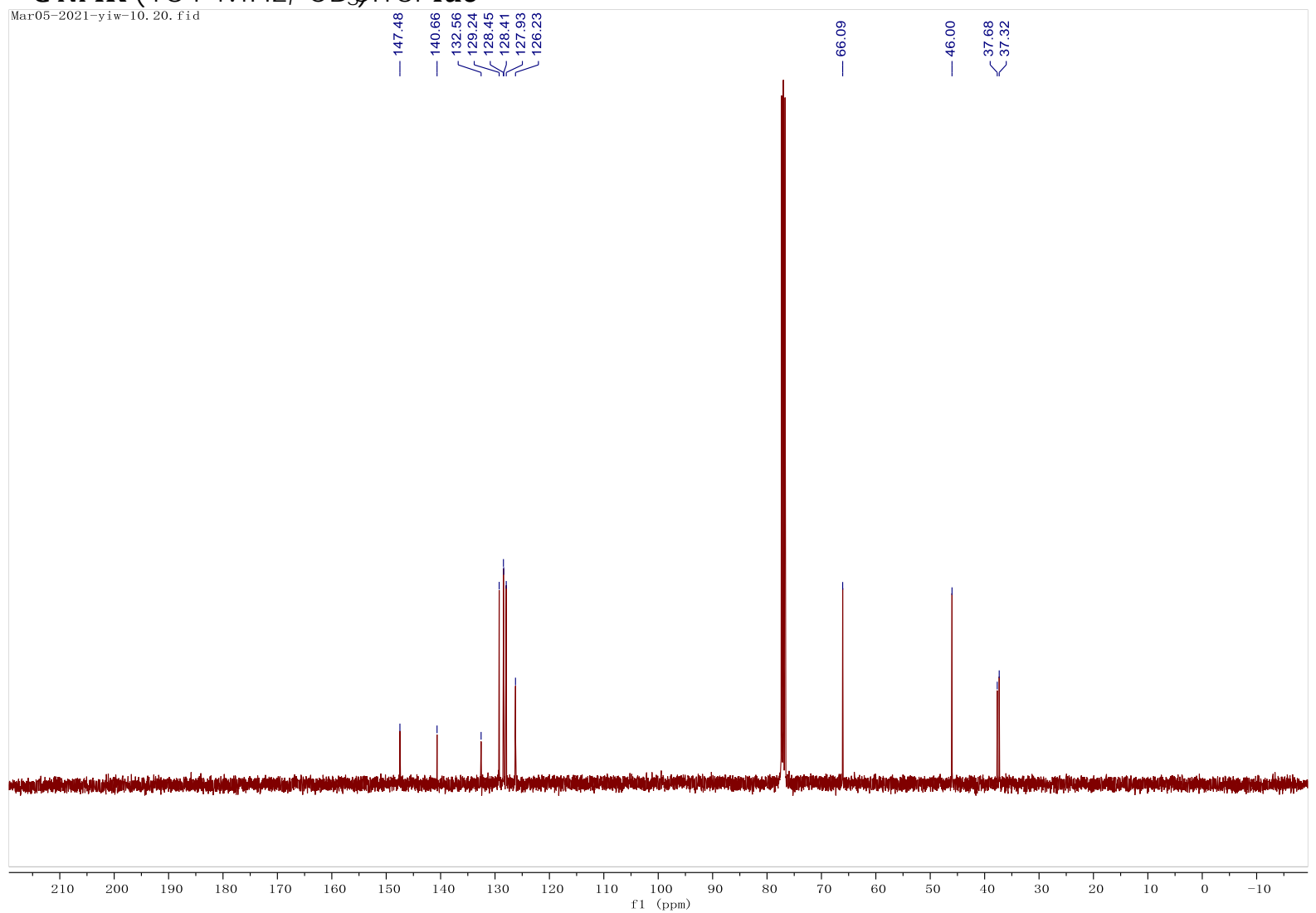
1H NMR (400 MHz, CDG) for 4ad

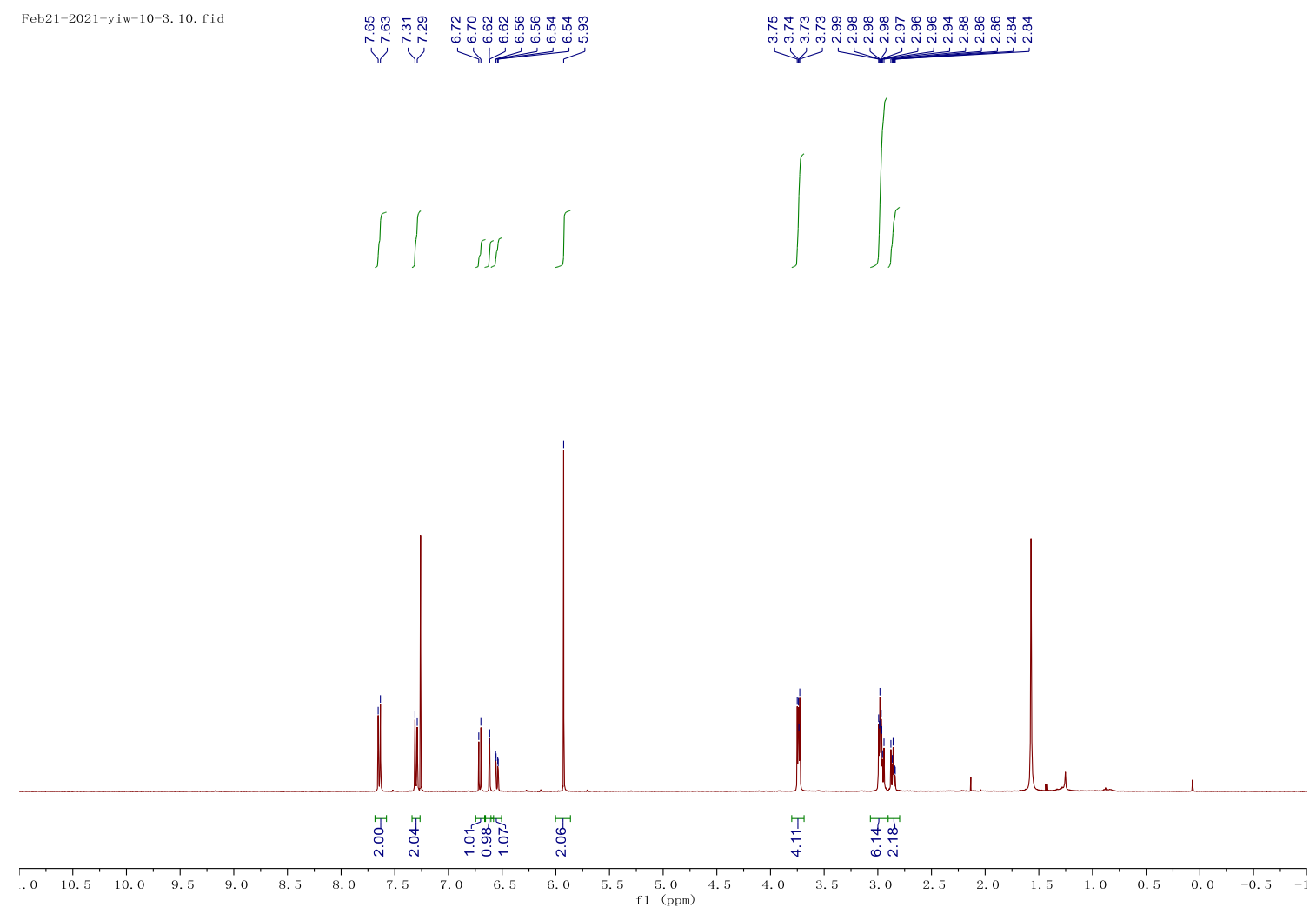

13C NMR (101 MHz, CDG) for 4ad

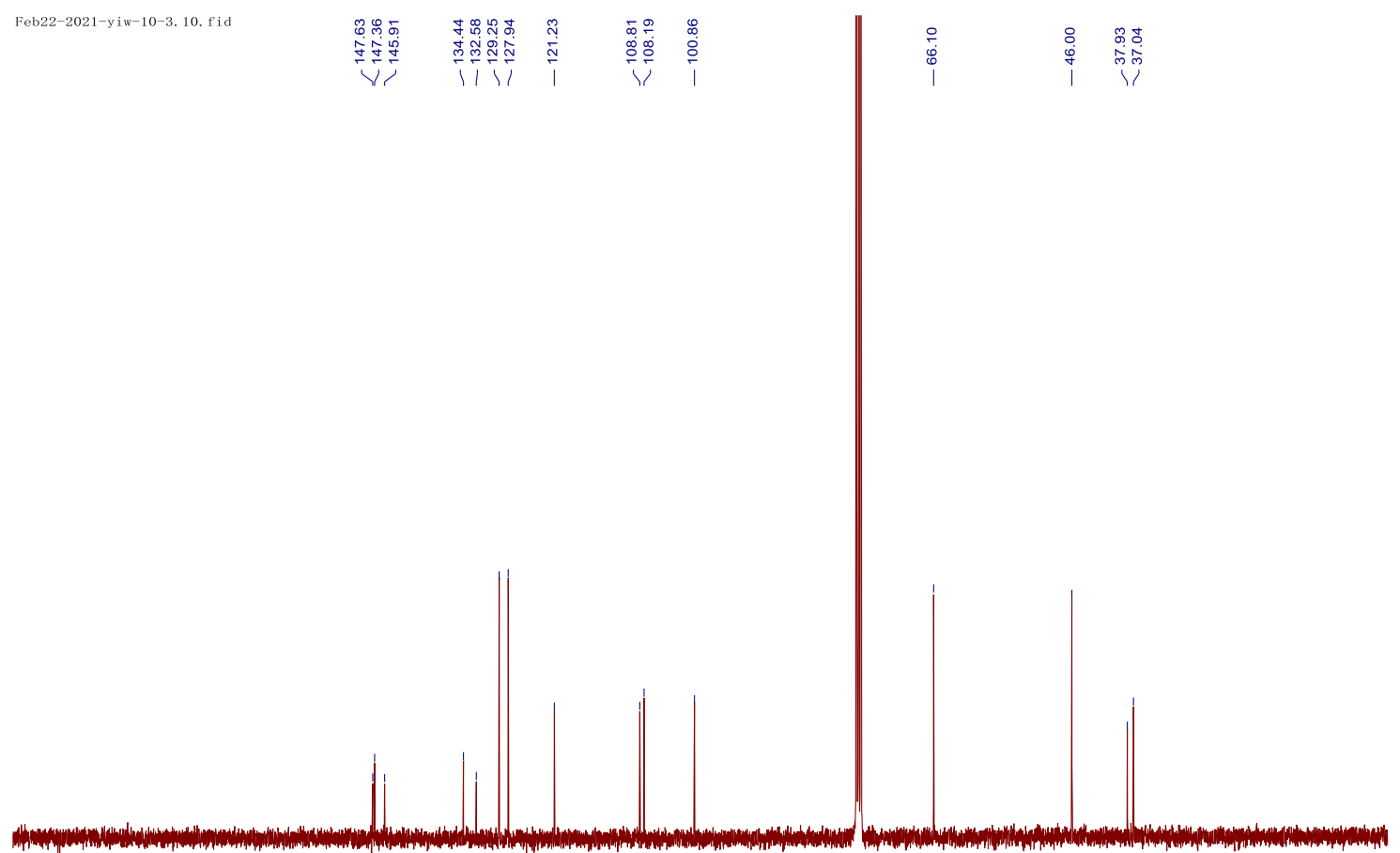

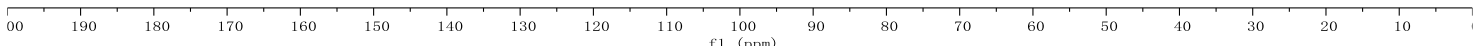


1H NMR (500 MHz, CDG) for 4ae

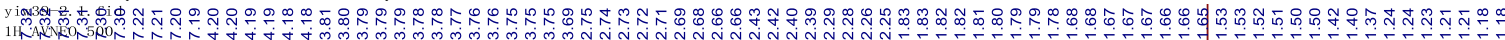

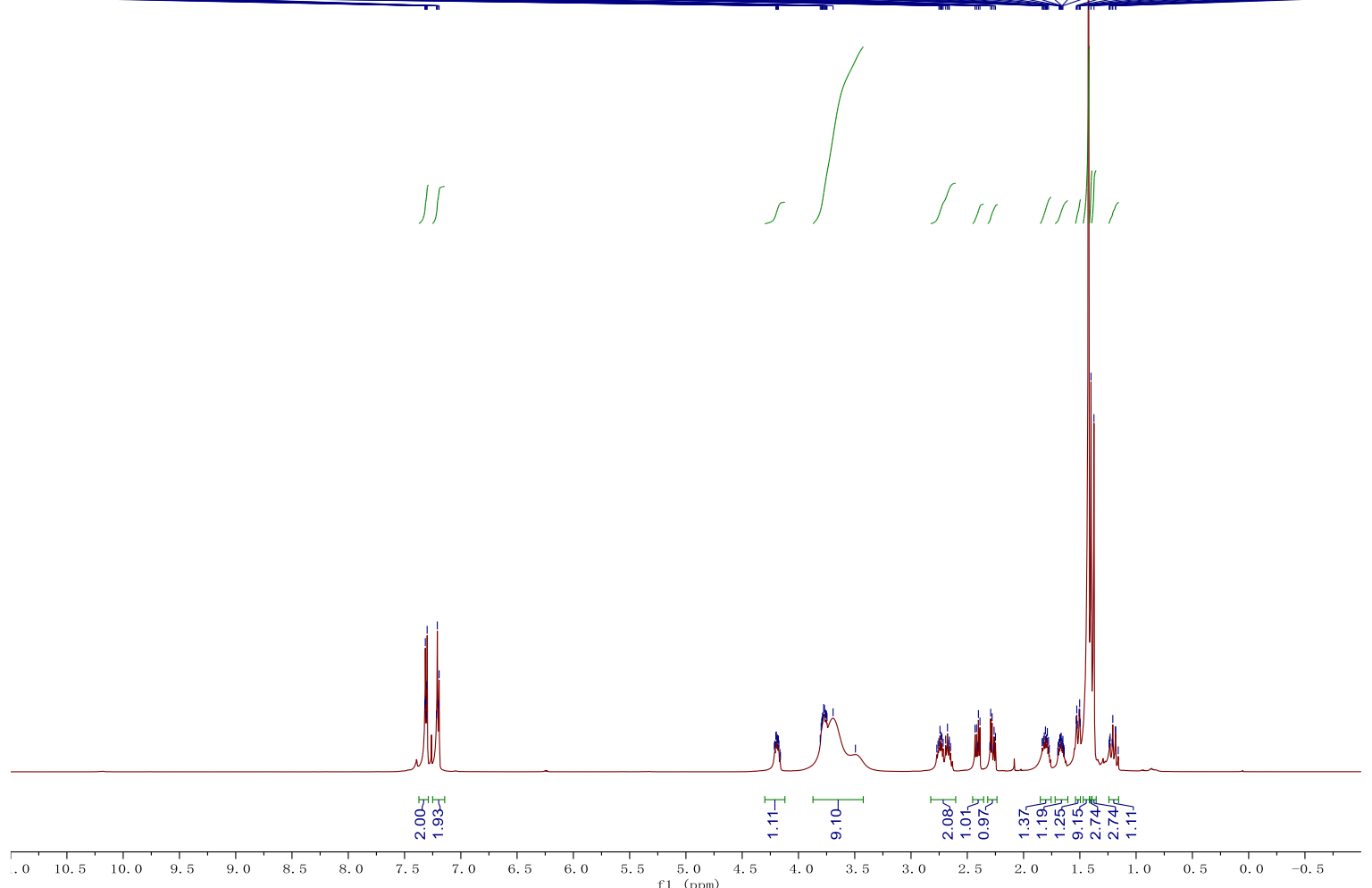

${ }^{13} \mathbf{C}$ NMR (126 MHz, CDG) for 4ae

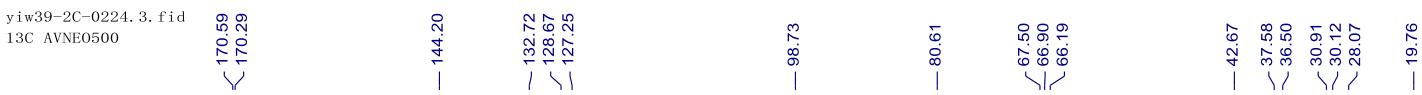

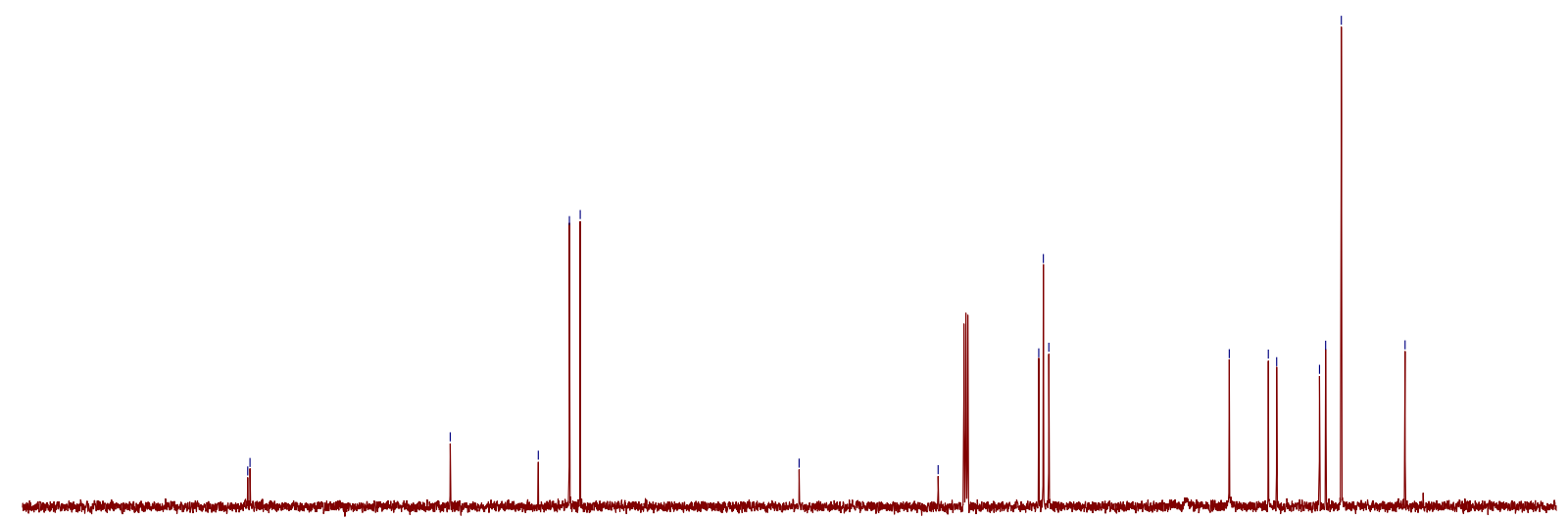

00

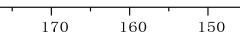

130

100
$(\mathrm{ppm})$ 
1H NMR (500 MHz, CDG) for 4af

yiw39-3-H. 1. fid
1H AVNEO 500

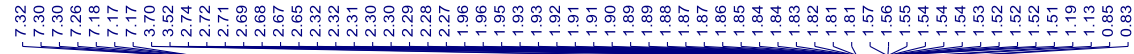
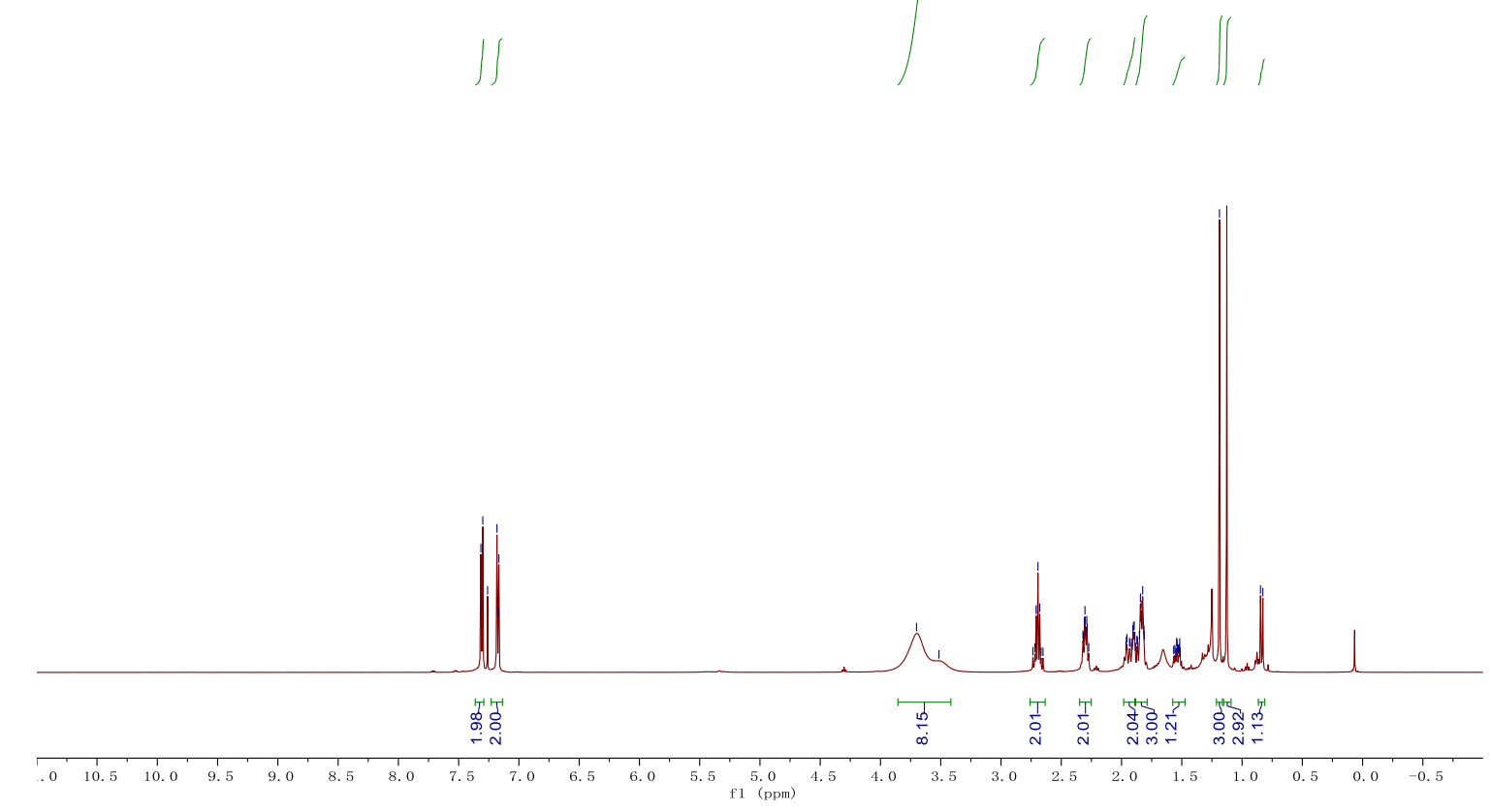

13C NMR (126 MHz, CDG) for 4af

13C AVNE0500

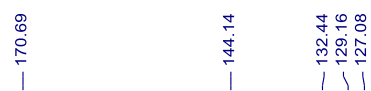

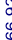

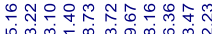

एु

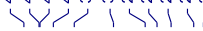

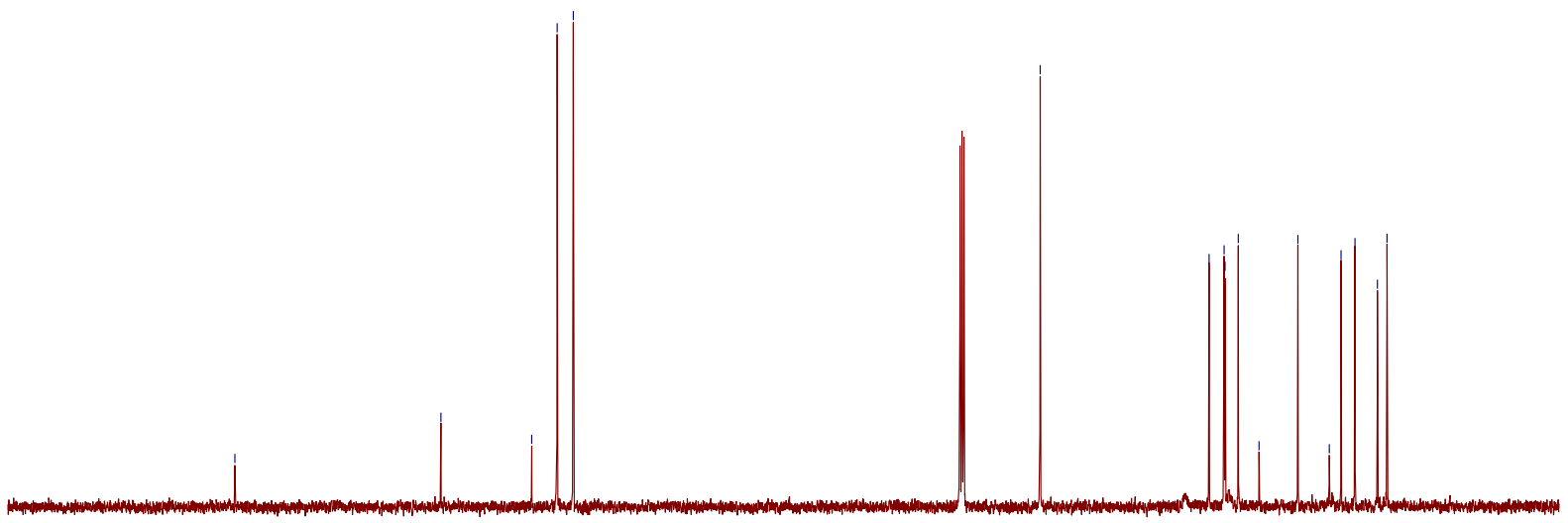

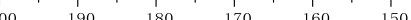
100 
${ }^{1} \mathbf{H}$ NMR (500 MHz, CDG) for $\mathbf{4 b}$

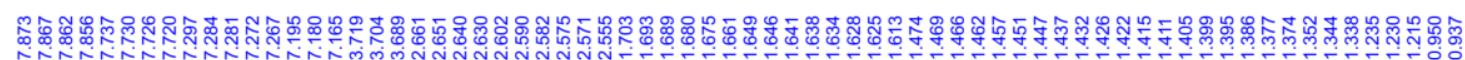
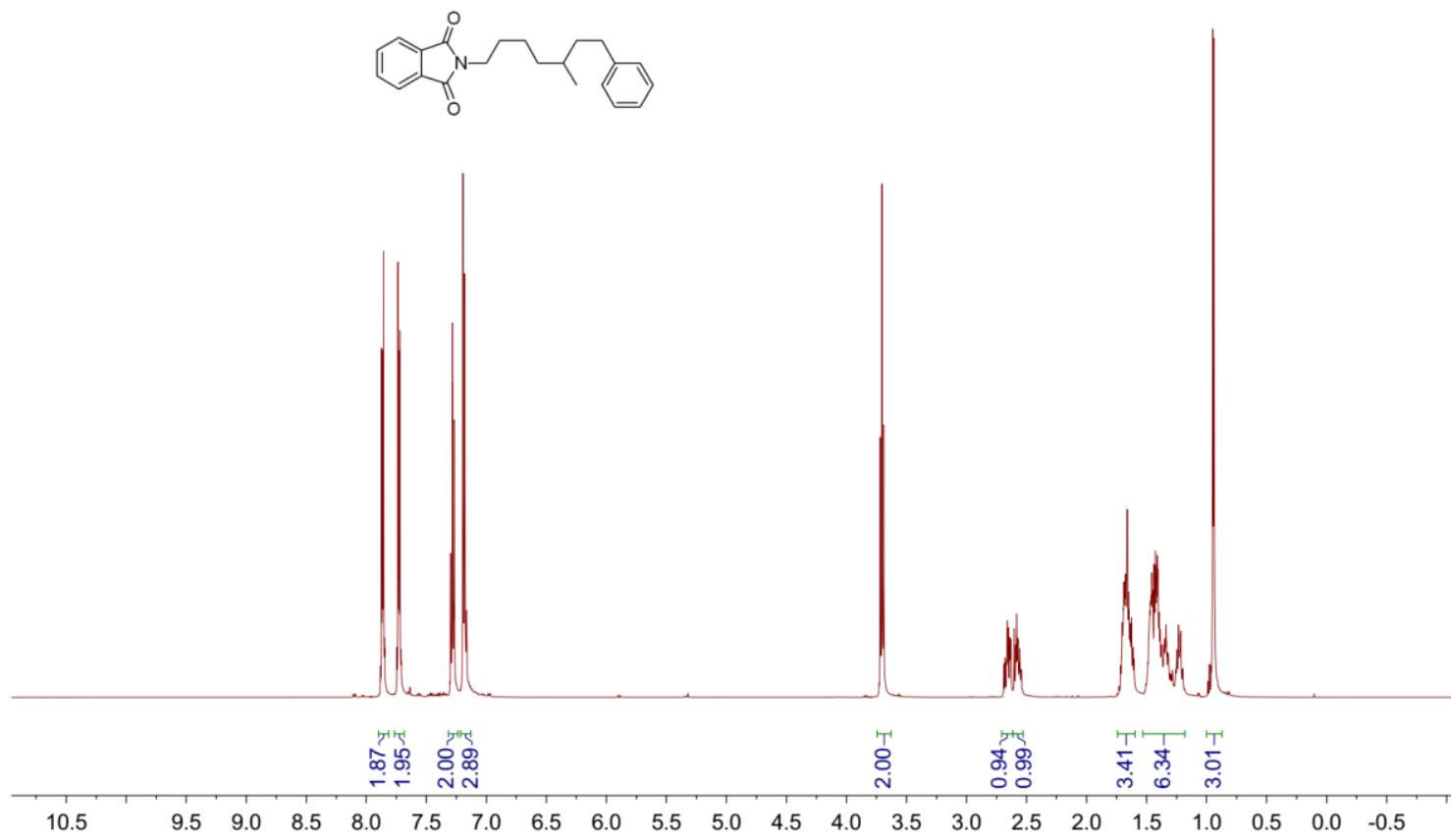

13C NMR (126 MHz, CDG) for $\mathbf{4 b}$
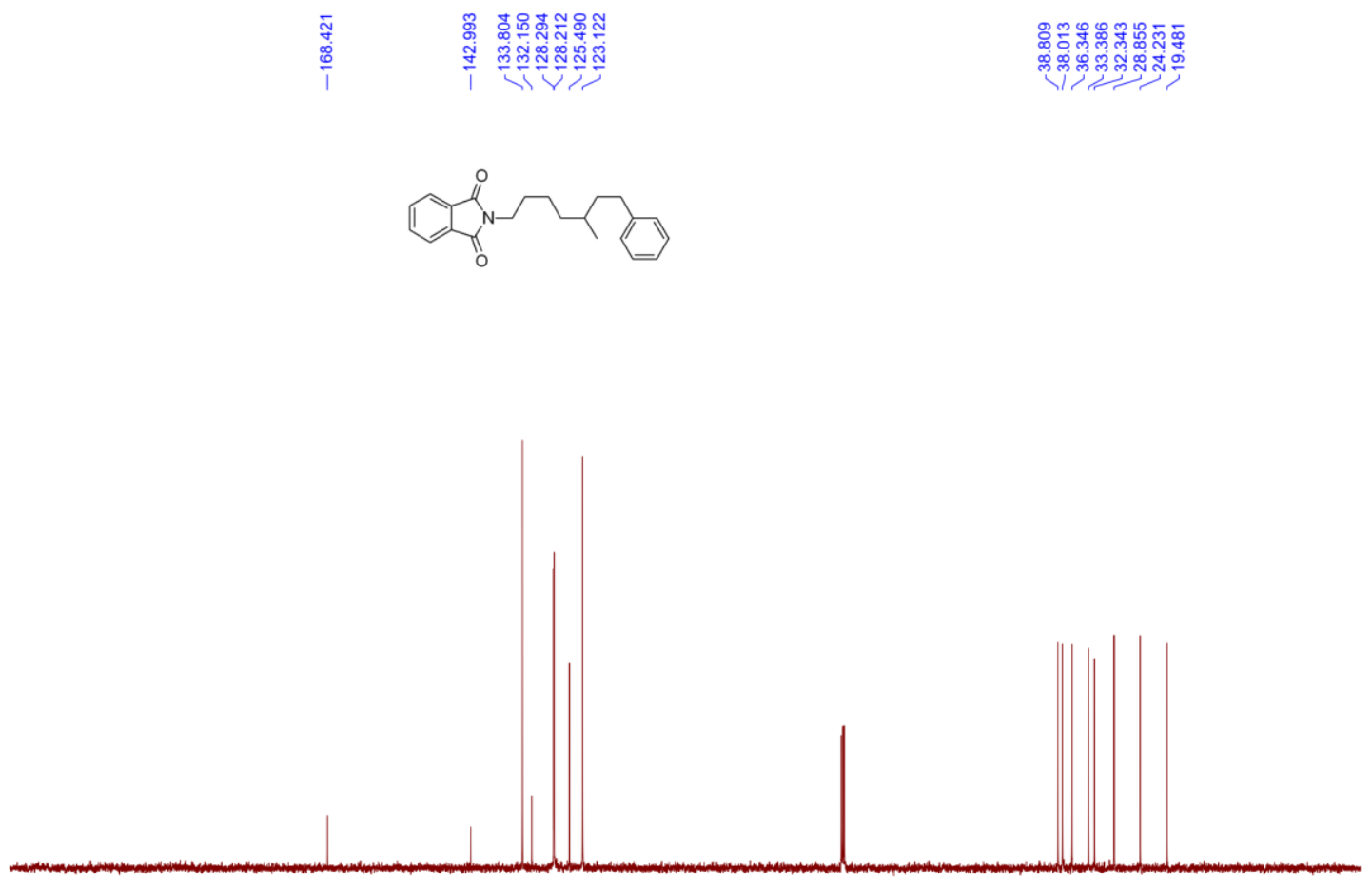

$\begin{array}{llllllllllllllllllllllllll}220 & 210 & 200 & 190 & 180 & 170 & 160 & 150 & 140 & 130 & 120 & 110 & 100 & 90 & 80 & 70 & 60 & 50 & 40 & 30 & 20 & 10 & 0 & -10\end{array}$ 
1H NMR (500 MHz, CDG) for $4 \mathbf{a g}$

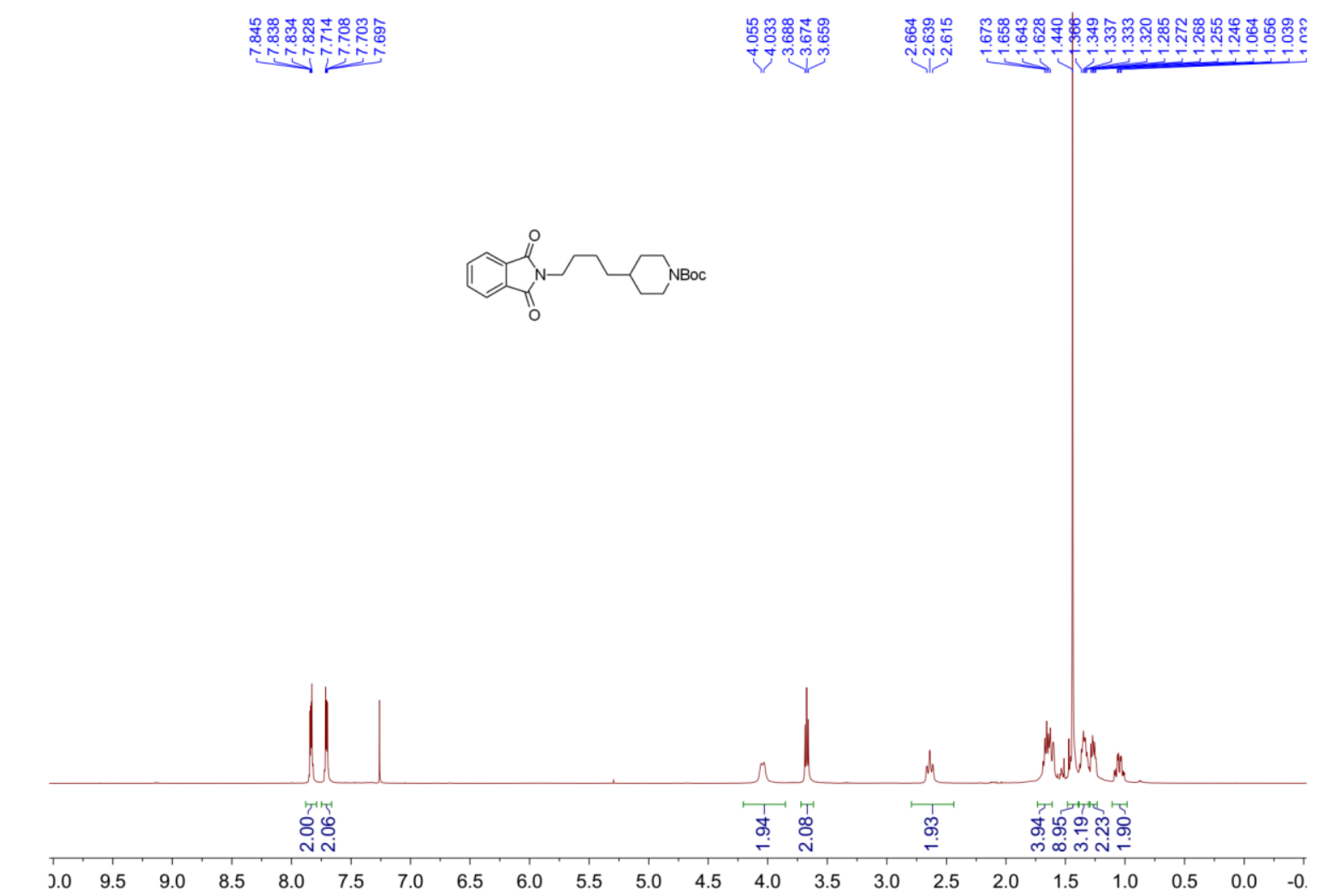

13C NMR (126 MHz, CDG) for $4 \mathbf{a g}$

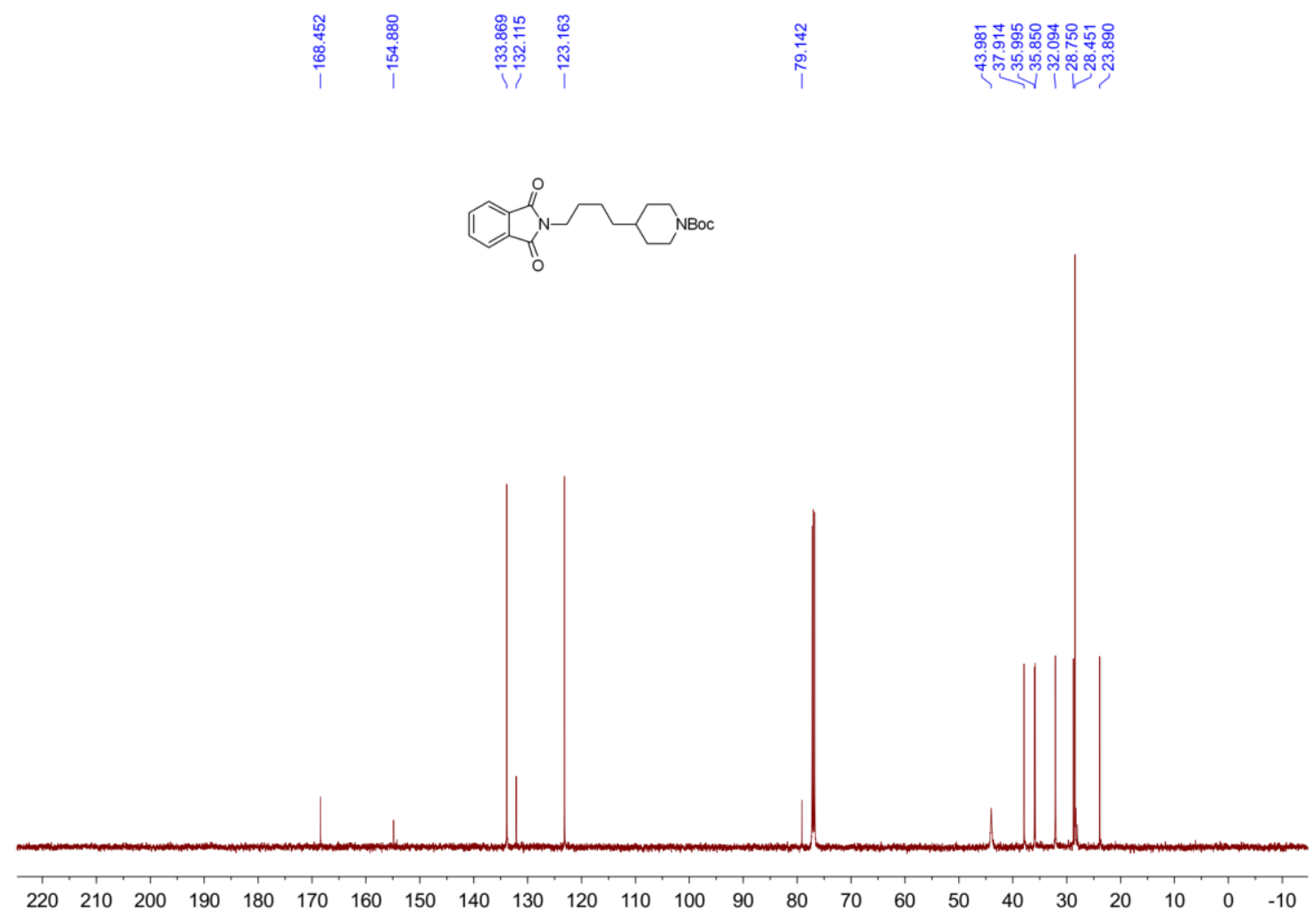


1H NMR (500 MHz, CDG) for 4ah

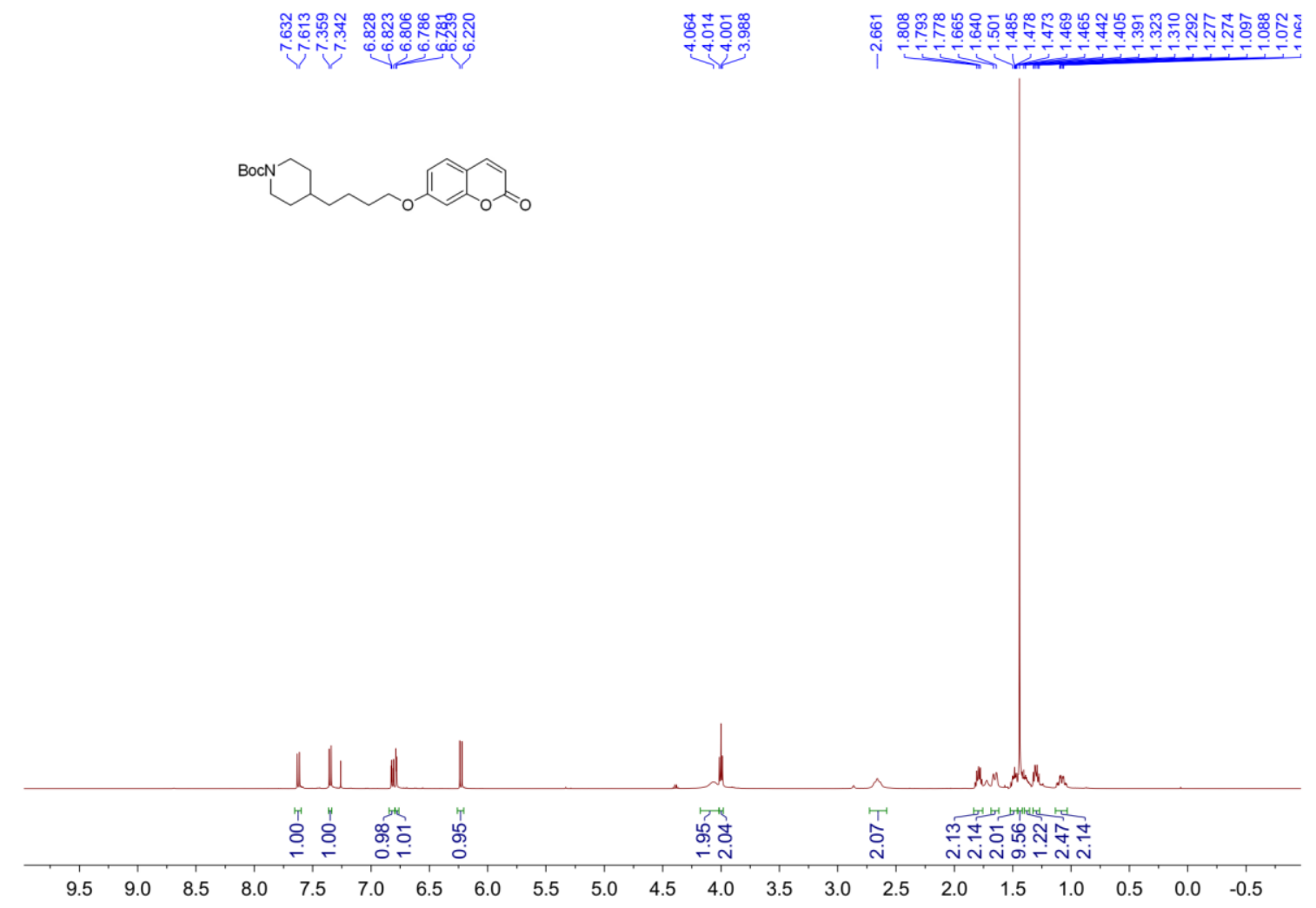

13C NMR (126 MHz, CDG) for 4ah
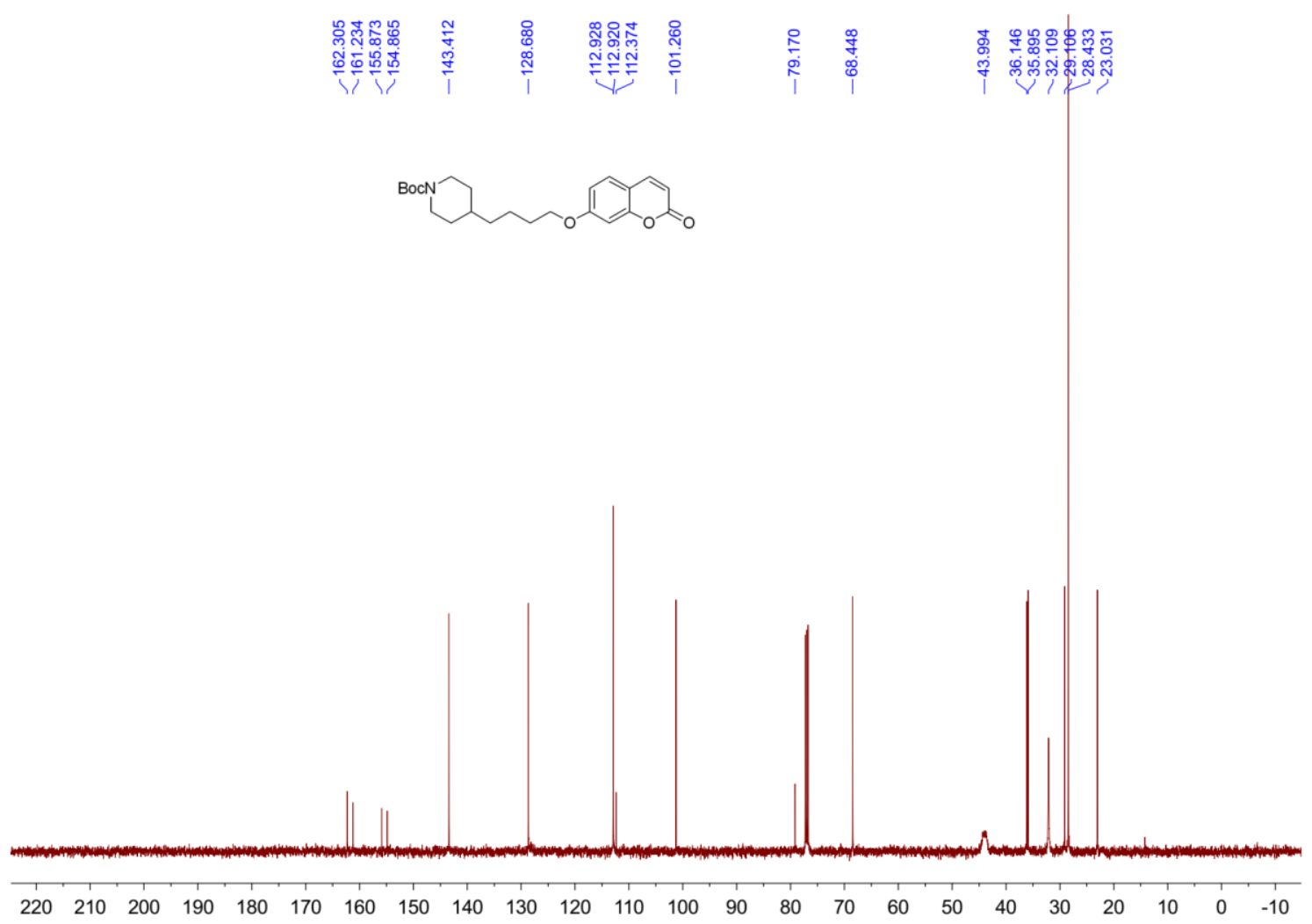
1H NMR (500 MHz, CDG) for 4ai
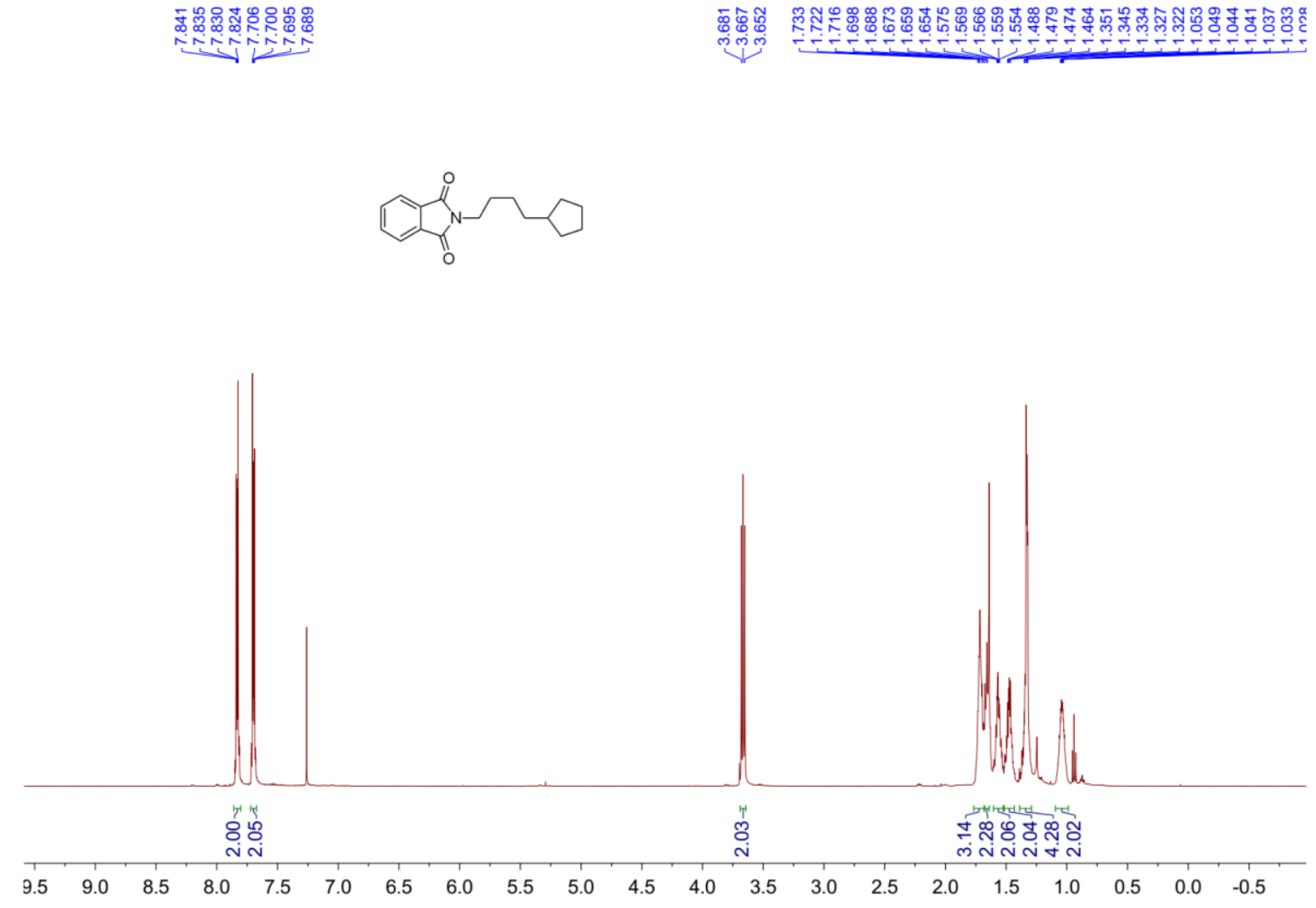

13C NMR (126 MHz, CDG) for 4ai

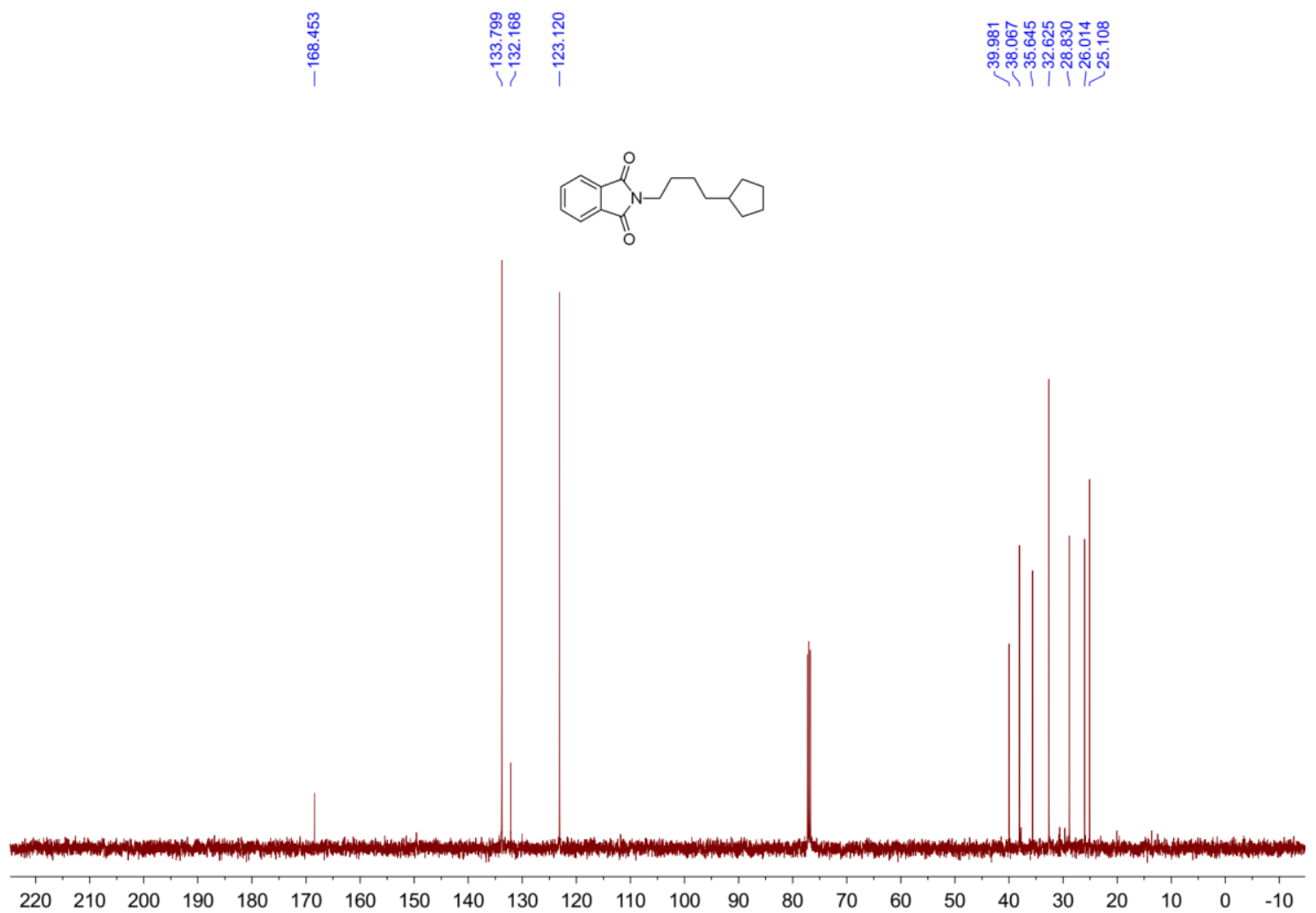


1H NMR (500 MHz, CDG) for 4aj

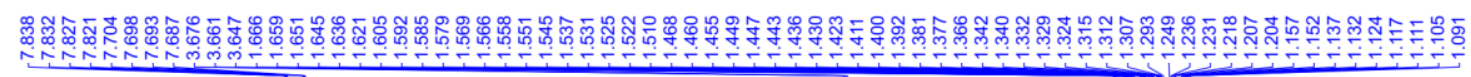

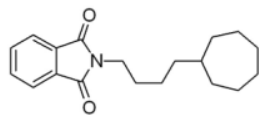

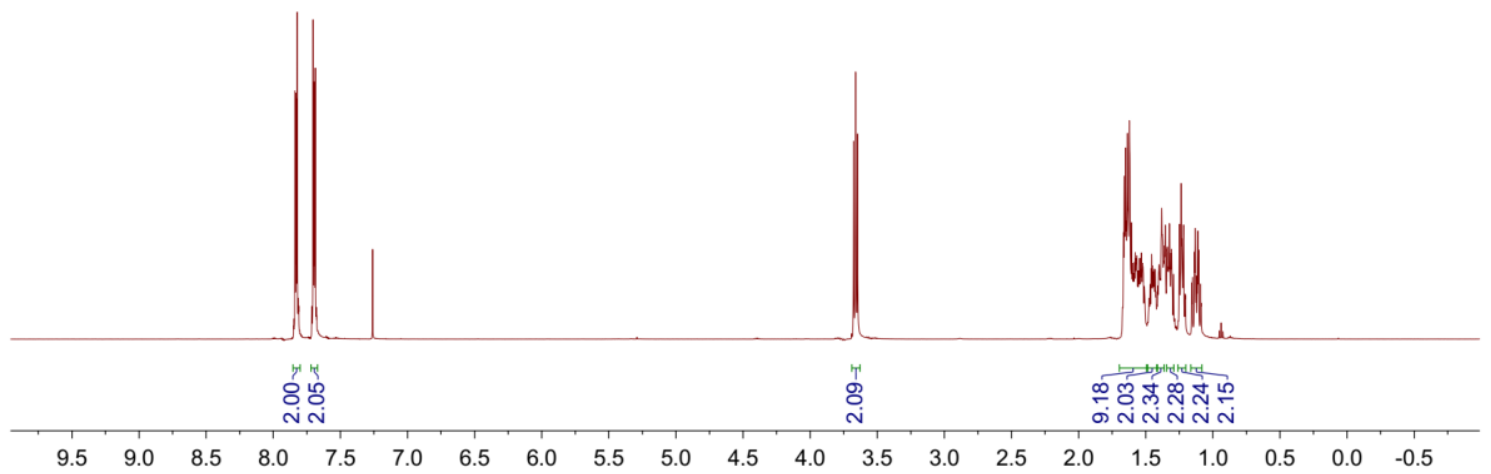

13C NMR (126 MHz, CDG) for 4aj

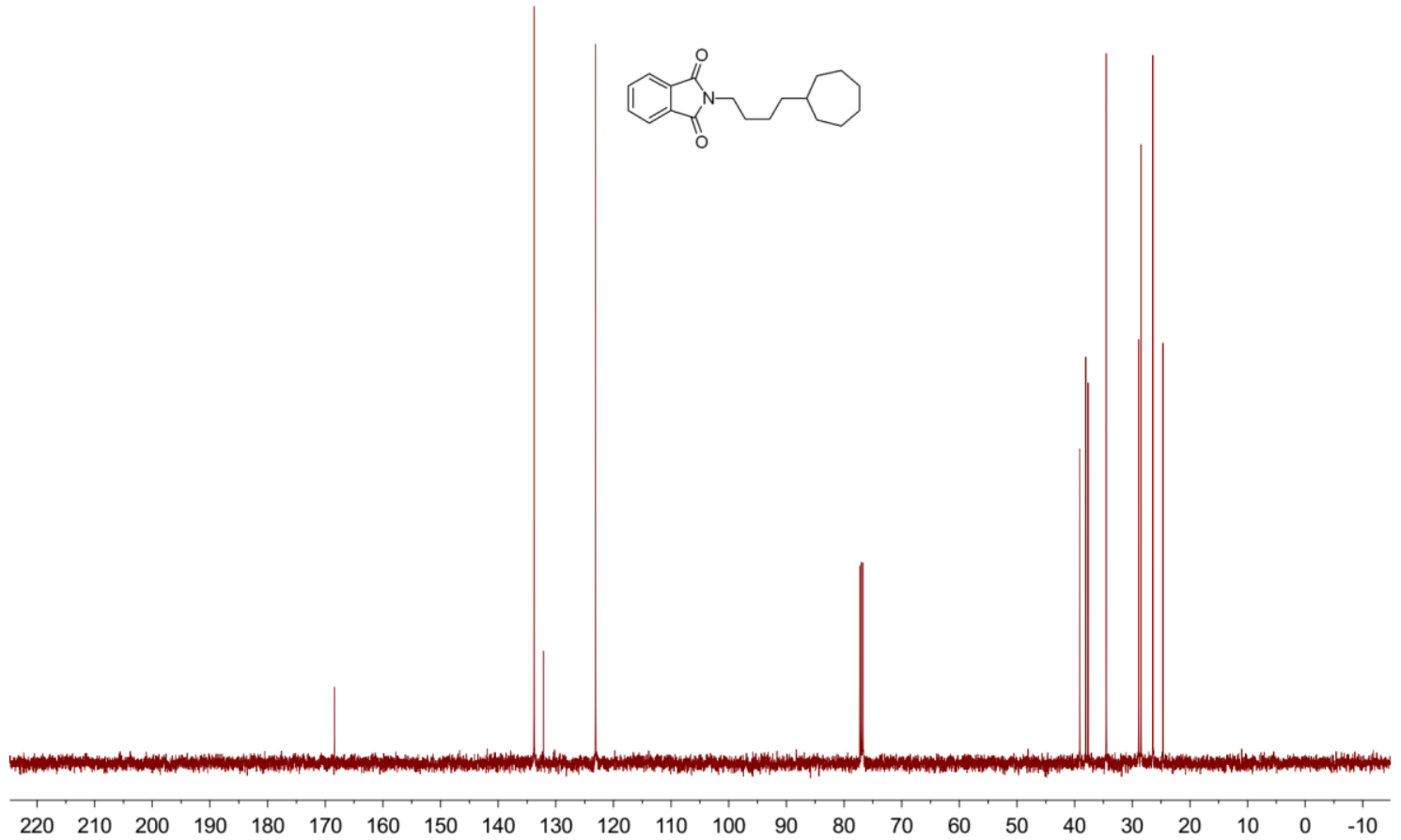


1H NMR (500 MHz, CDG) for $4 \mathbf{a k}$
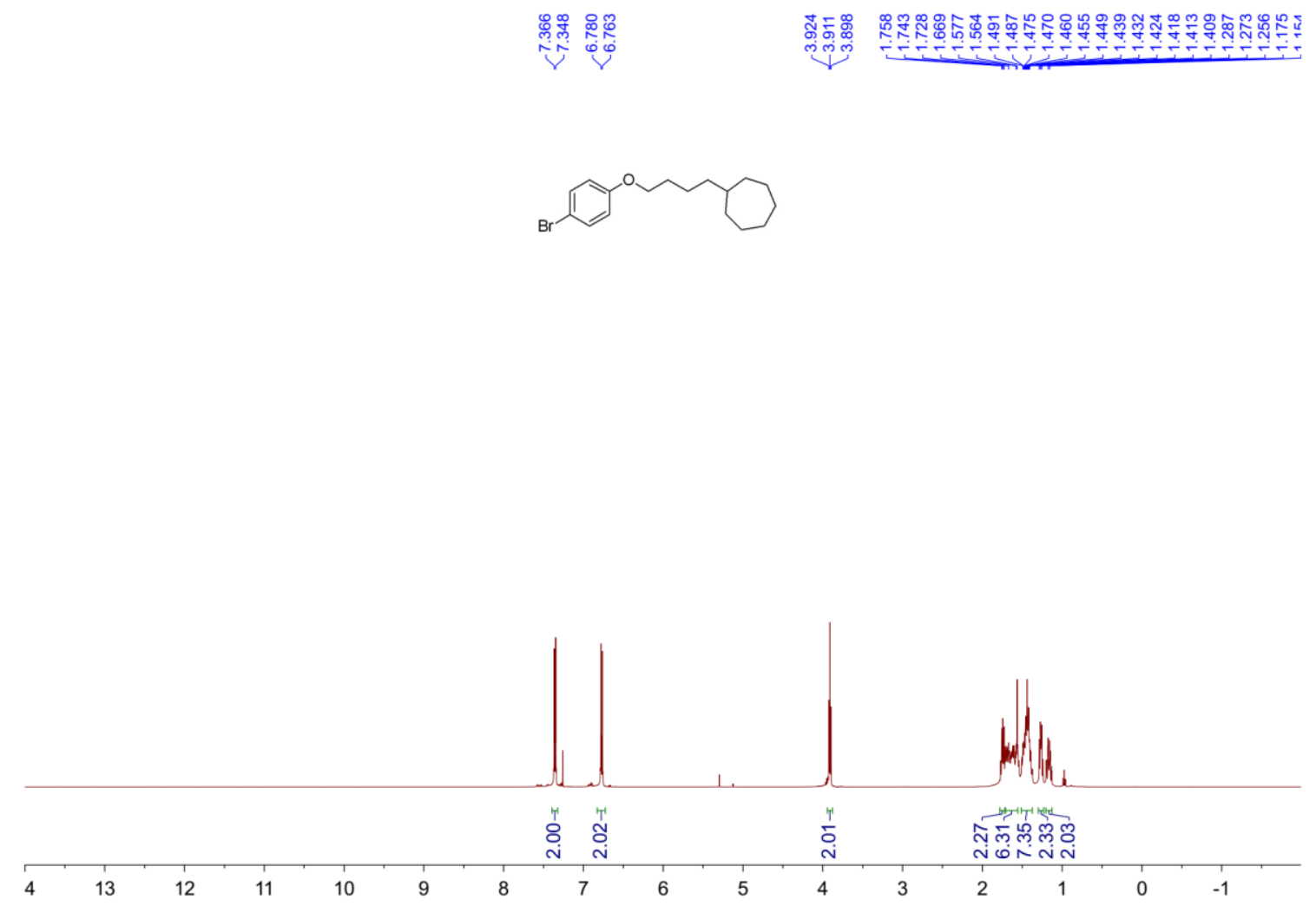

13C NMR (126 MHz, CDG) for 4ak
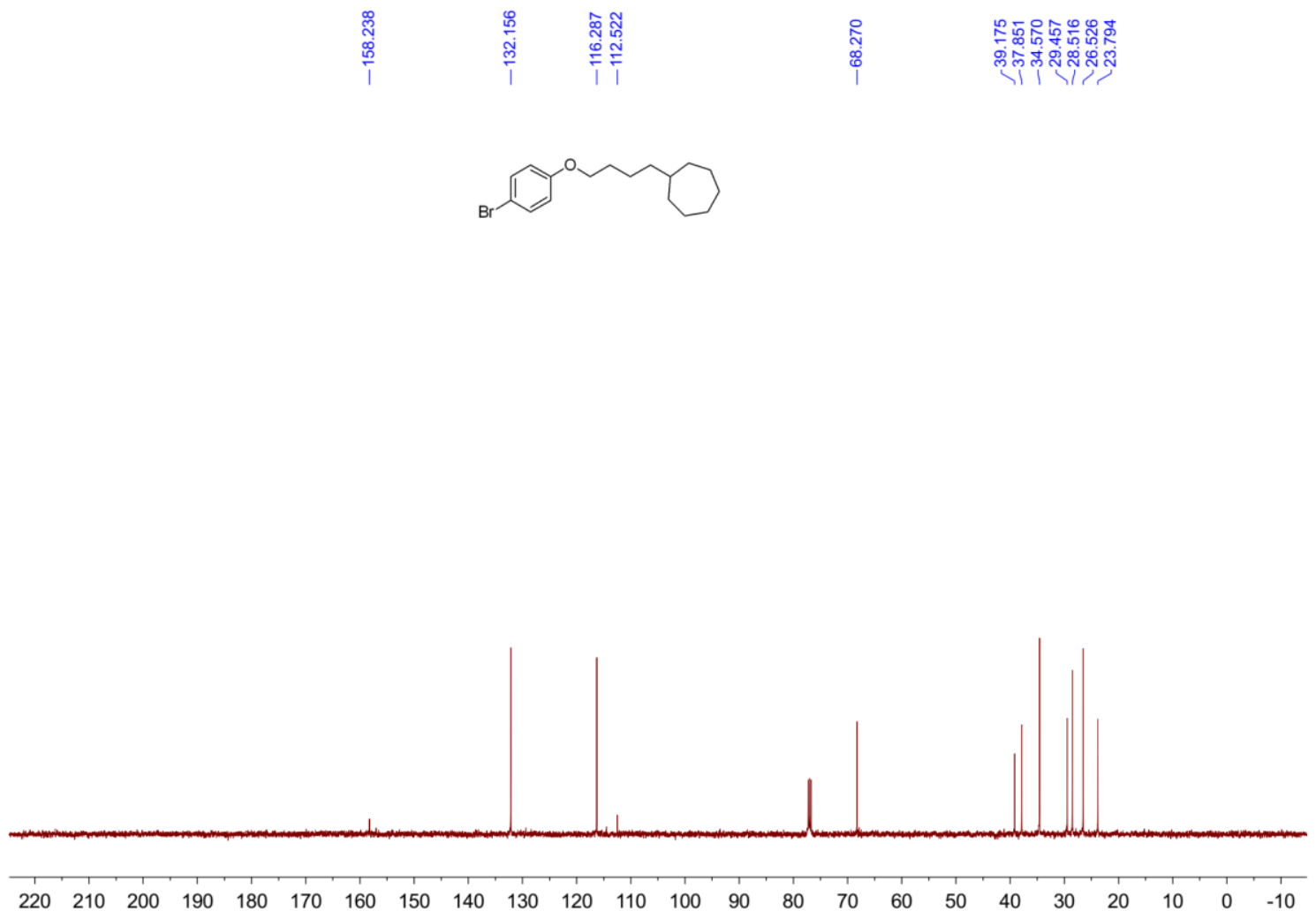
1H NMR (500 MHz, CDG) for 4al

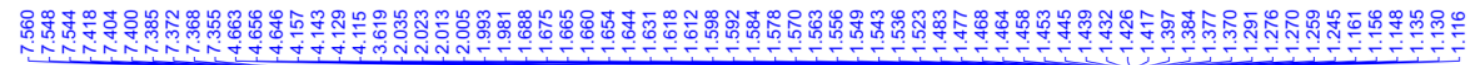

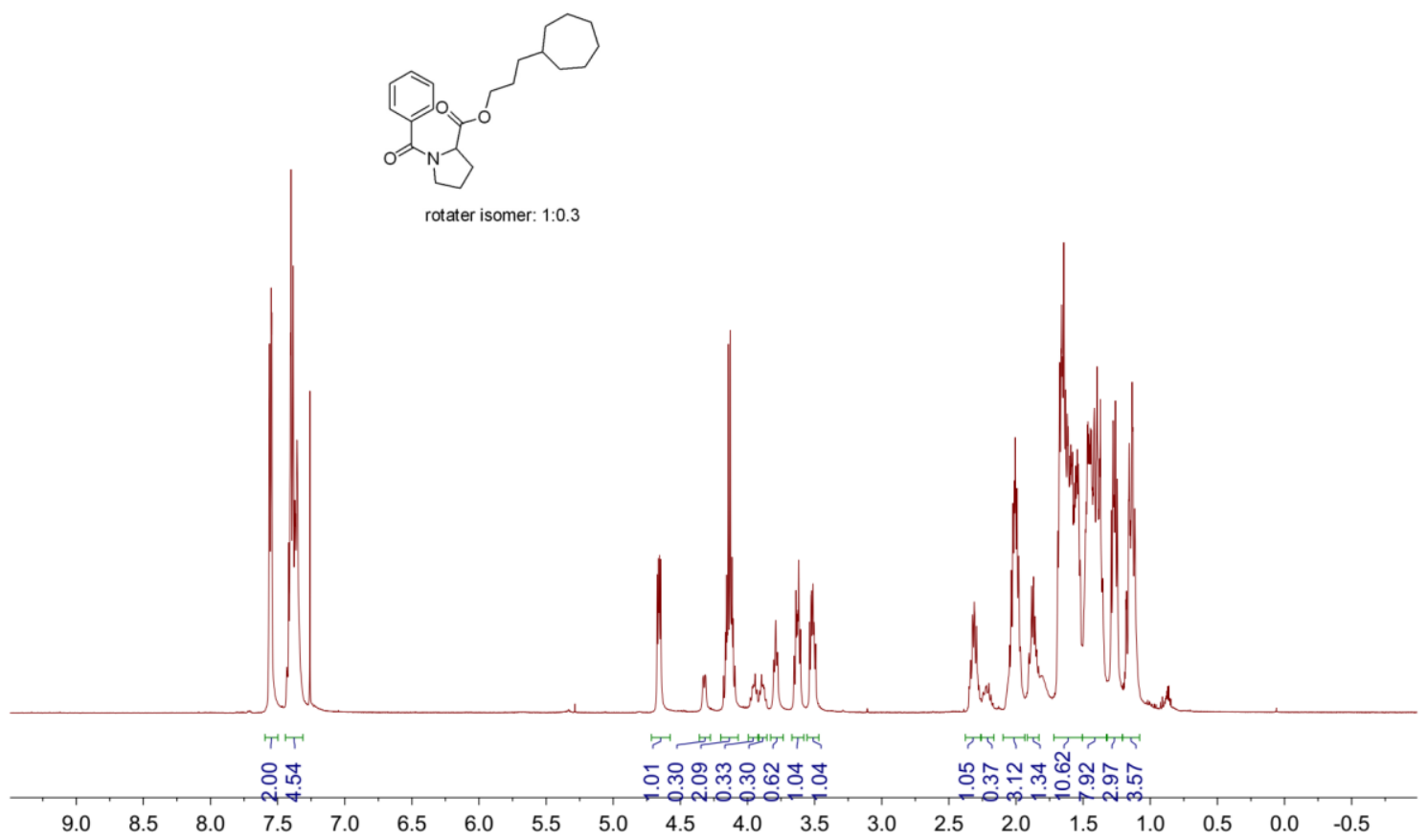

${ }^{13}$ C NMR (126 MHz, CDG) for 4al

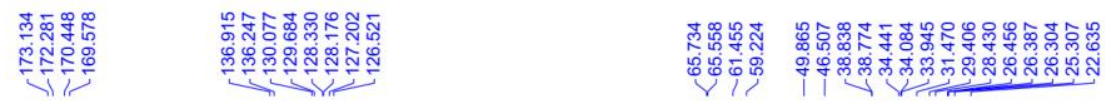

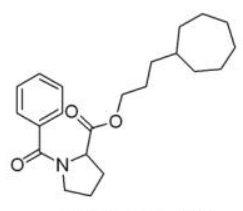

rotater isomer: $1: 0.3$

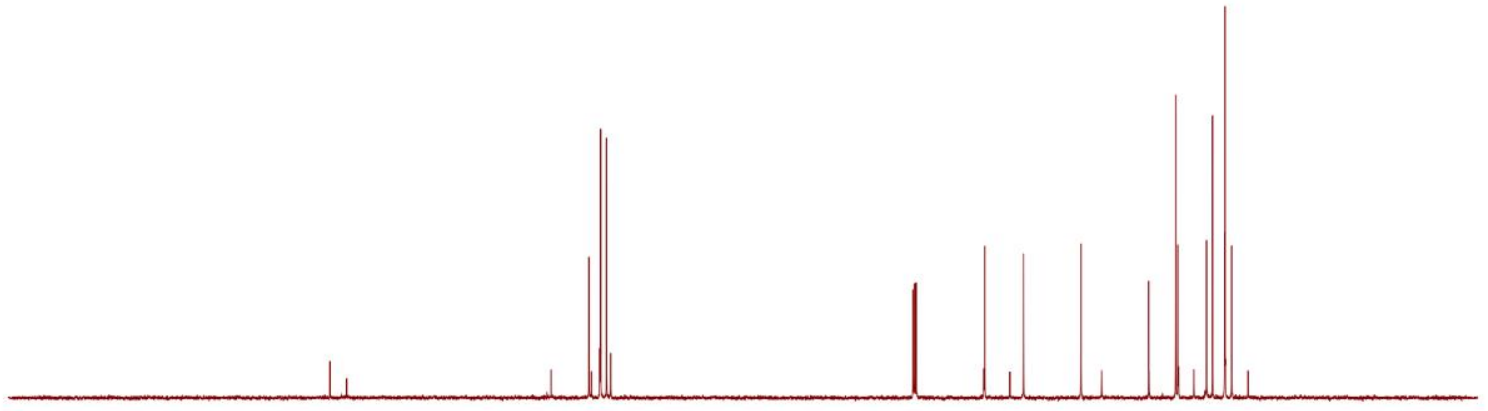

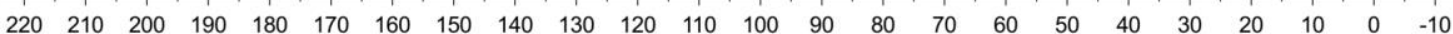


1H NMR (500 MHz, CDG) for 4am

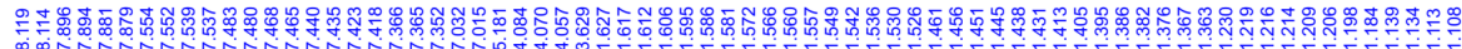
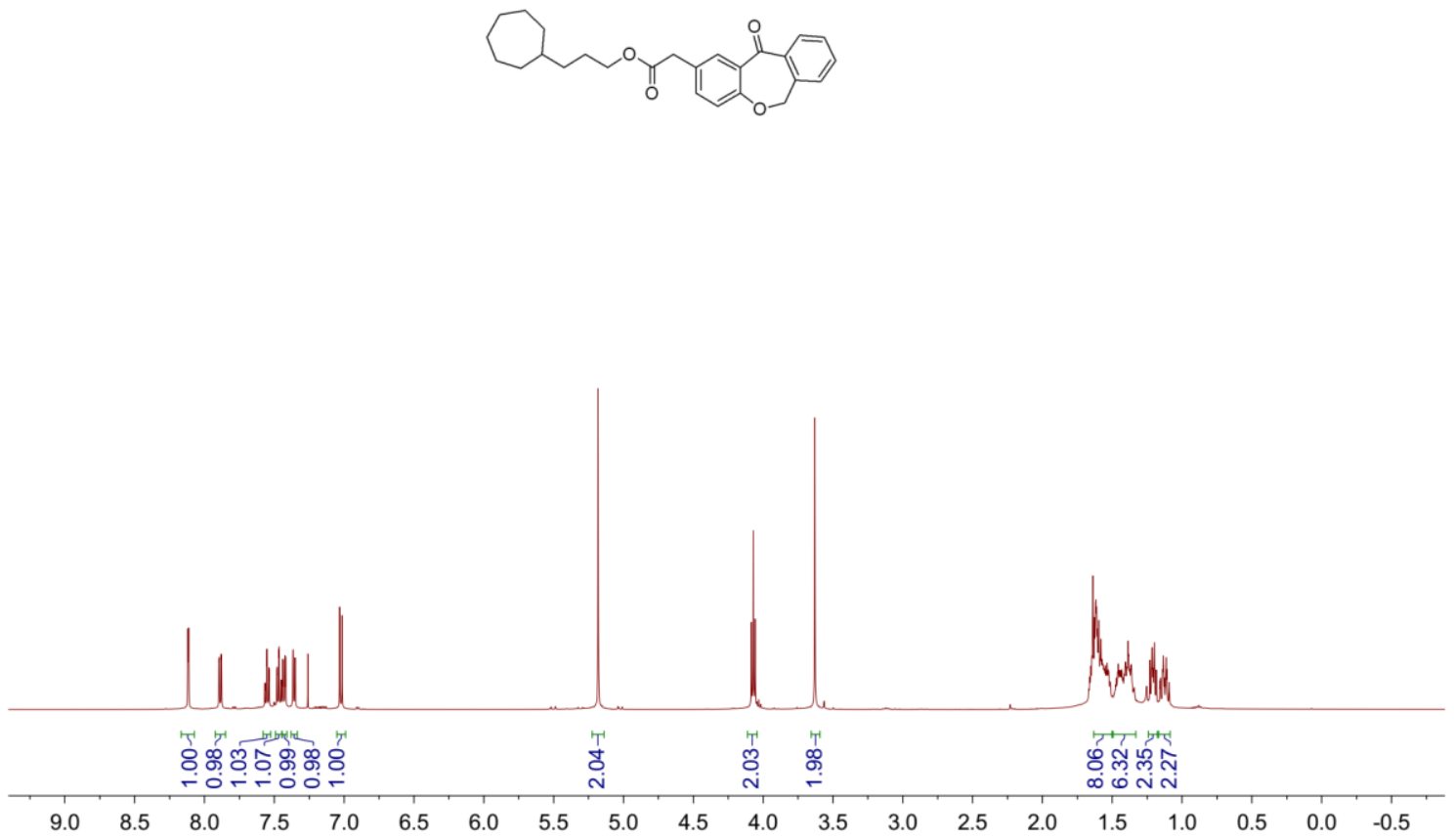

13C NMR (126 MHz, CDG) for 4am

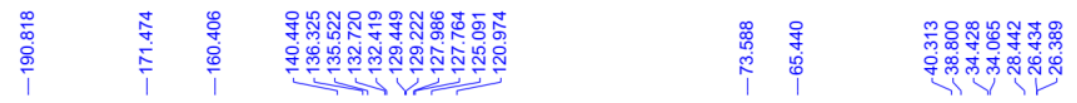
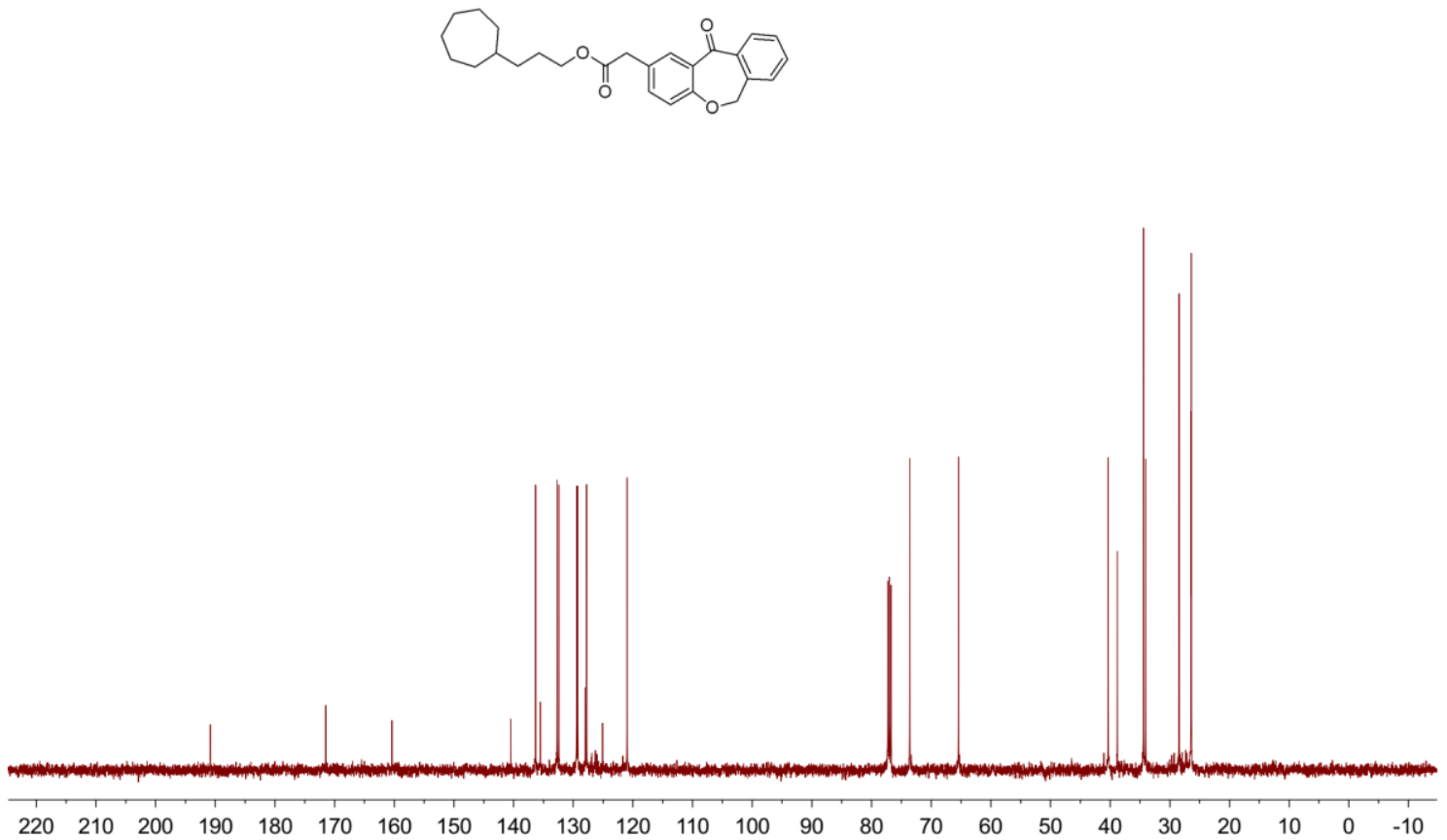
1H NMR (500 MHz, CDG) for 4an
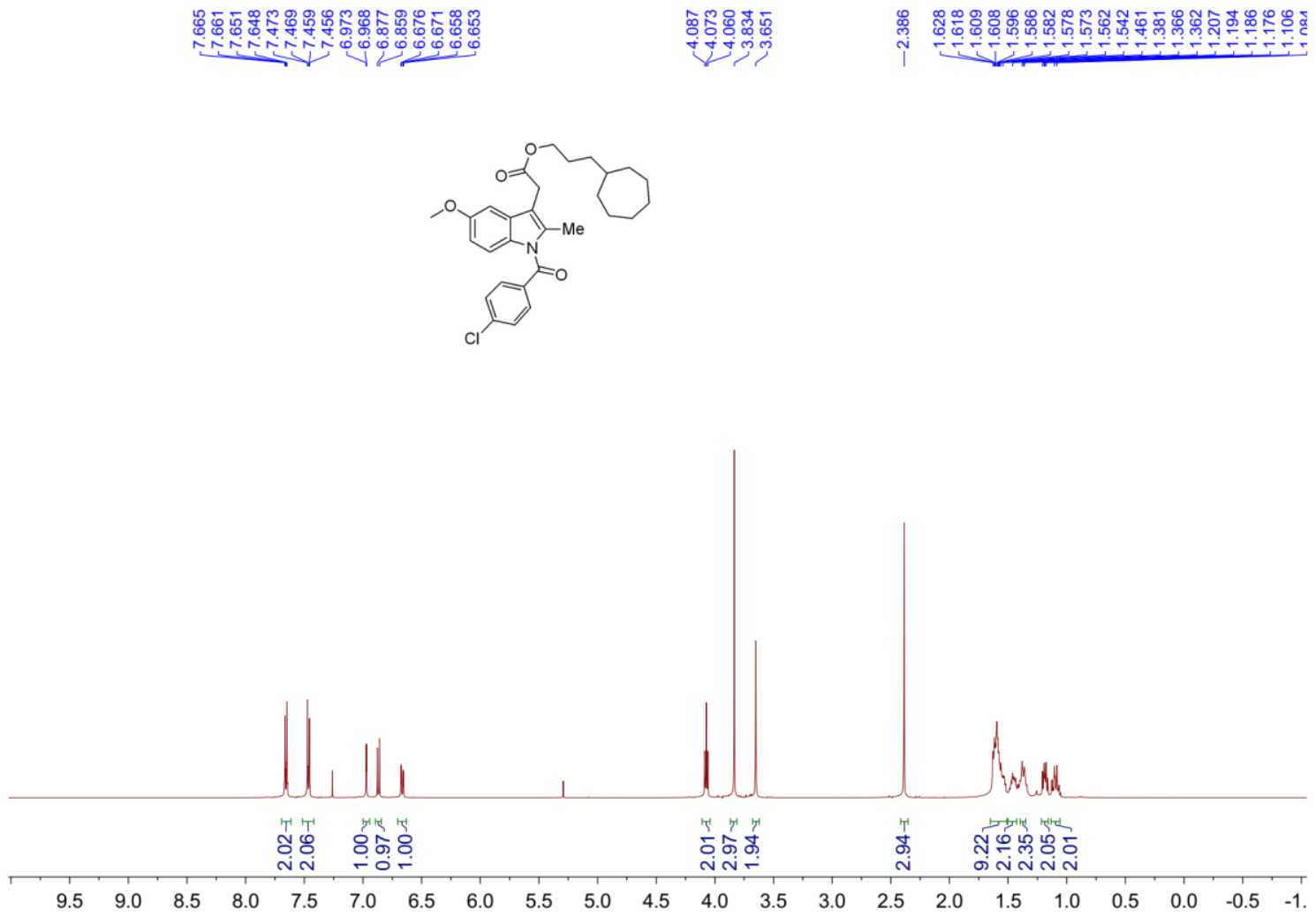

13C NMR (126 MHz, CDG) for 4an

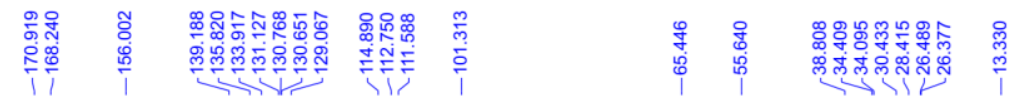
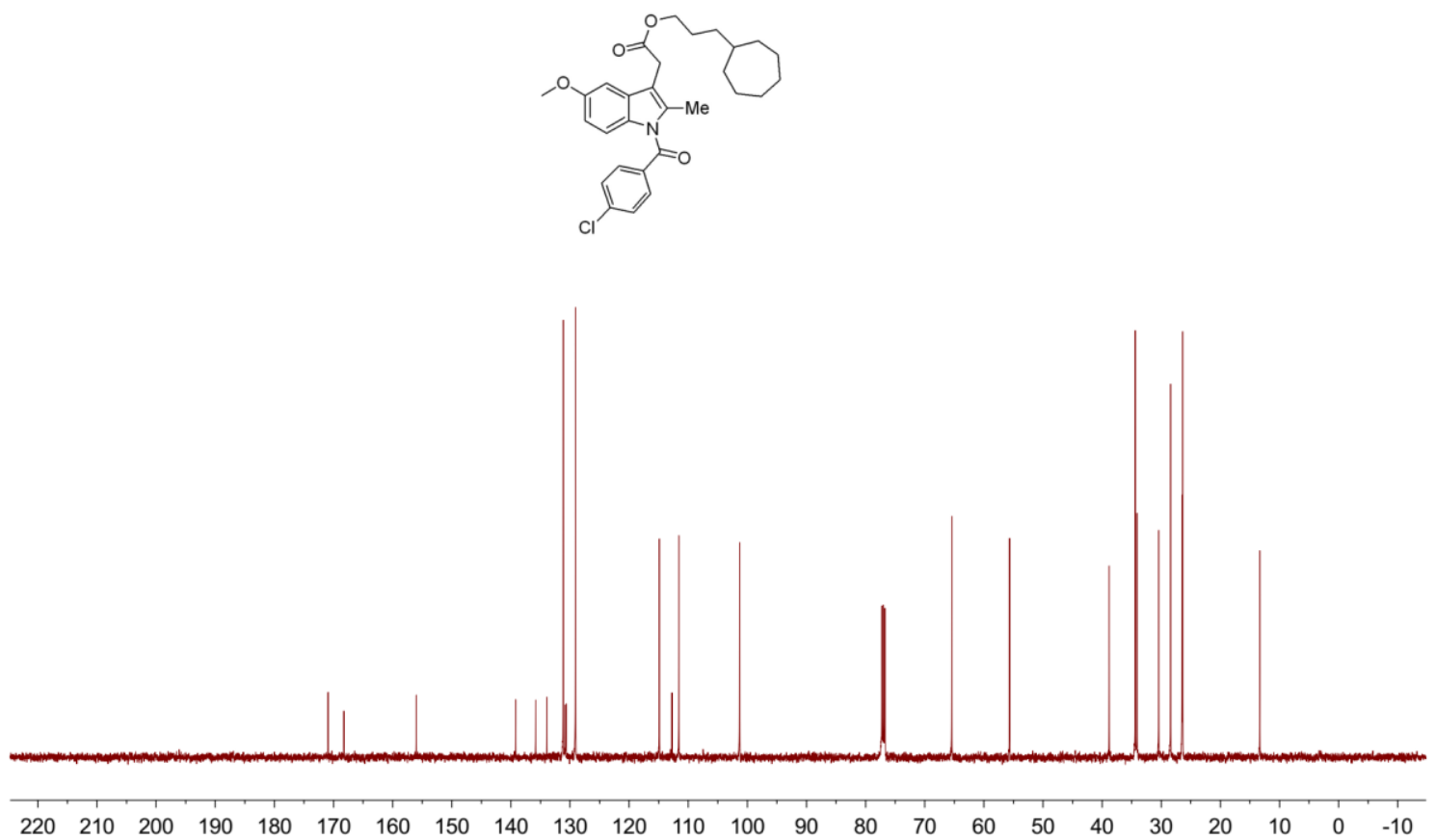
1H NMR (500 MHz, CDG) for 4 ao

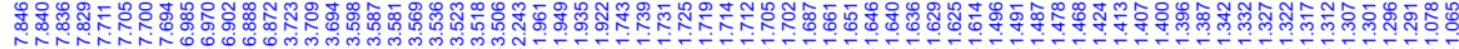
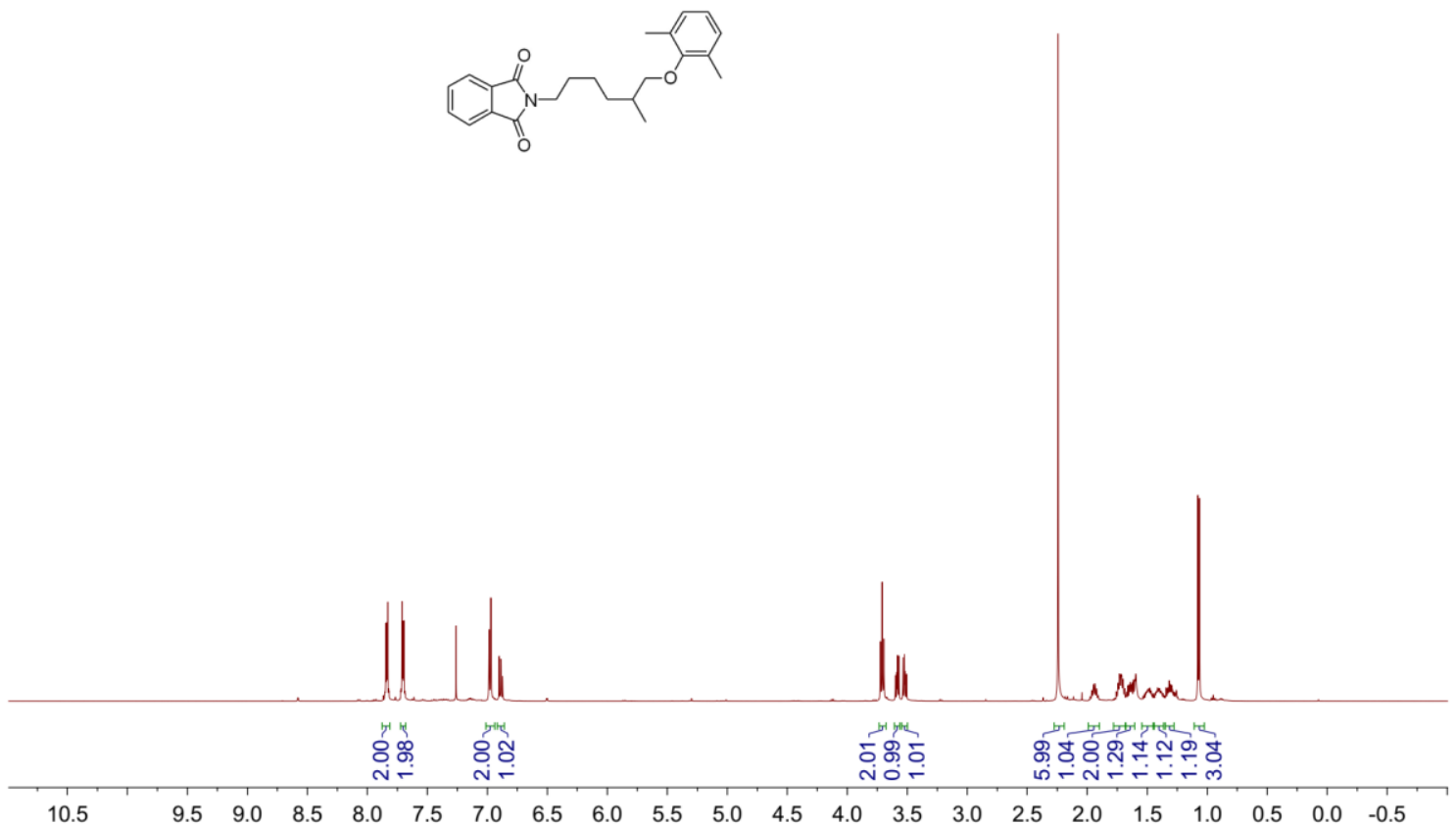

13C NMR (126 MHz, CDG) for 4ao

\begin{tabular}{|c|c|c|c|}
\hline 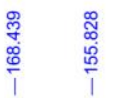 & 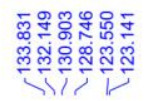 & $\begin{array}{l}\text { के } \\
\text { o } \\
0 \\
0\end{array}$ & 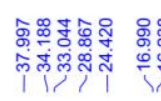 \\
\hline
\end{tabular}
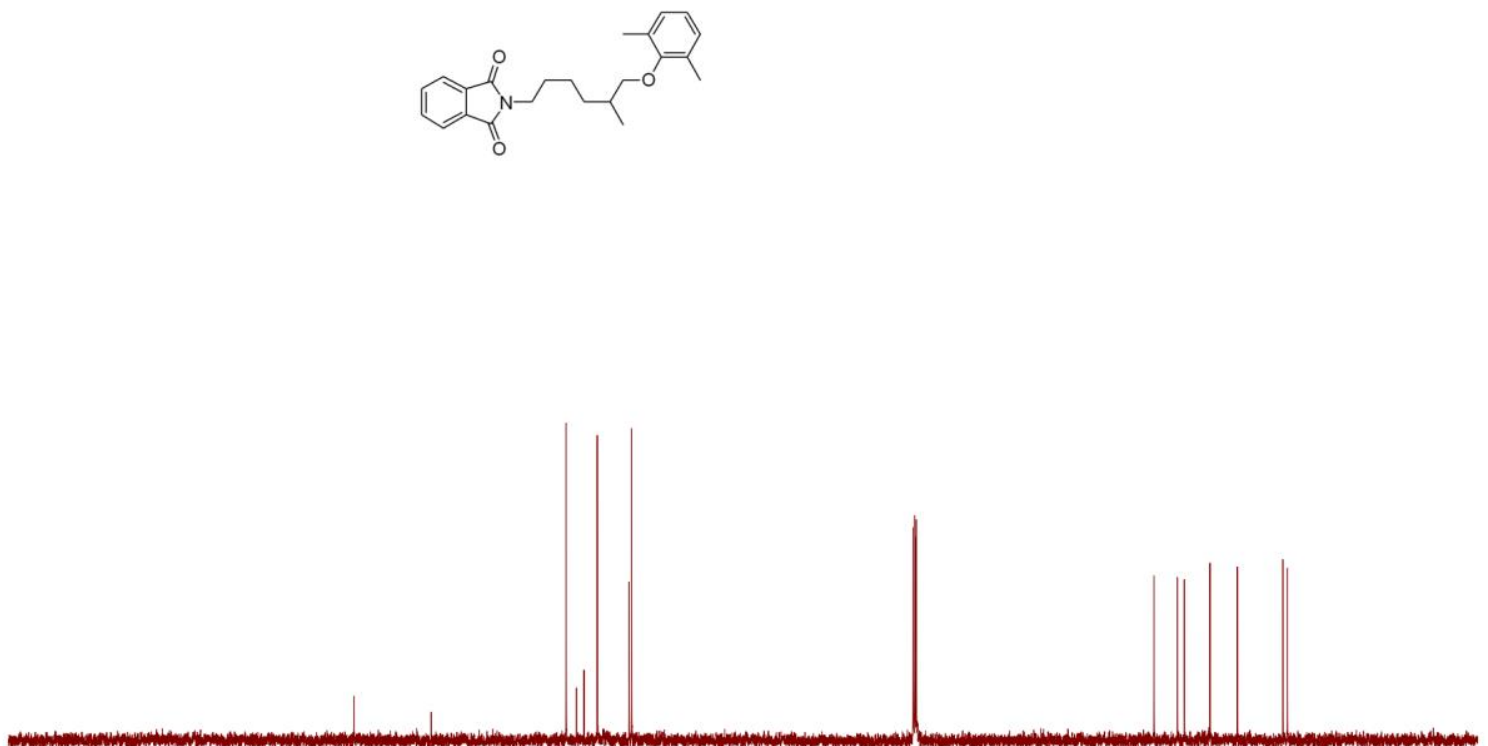

$\begin{array}{llllllllllllllllllllllll}220 & 210 & 200 & 190 & 180 & 170 & 160 & 150 & 140 & 130 & 120 & 110 & 100 & 90 & 80 & 70 & 60 & 50 & 40 & 30 & 20 & 10 & 0 & -10\end{array}$ 
1H NMR (500 MHz, CDG) for 4ap

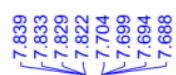

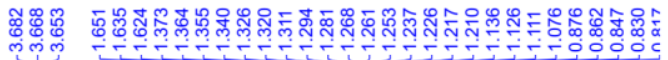

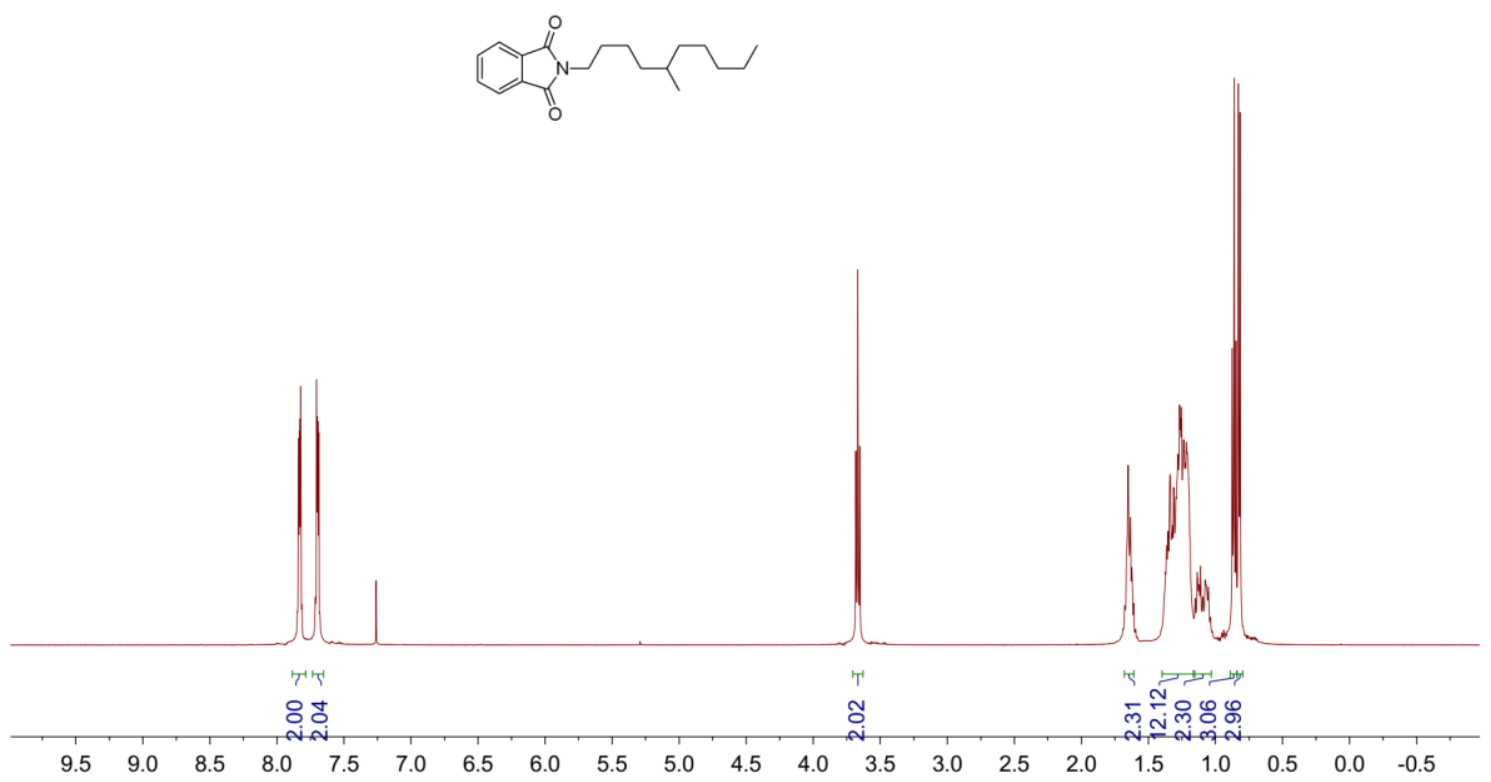

13C NMR (126 MHz, CDG) for 4ap

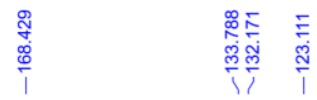

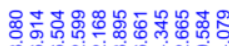

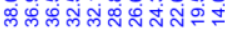
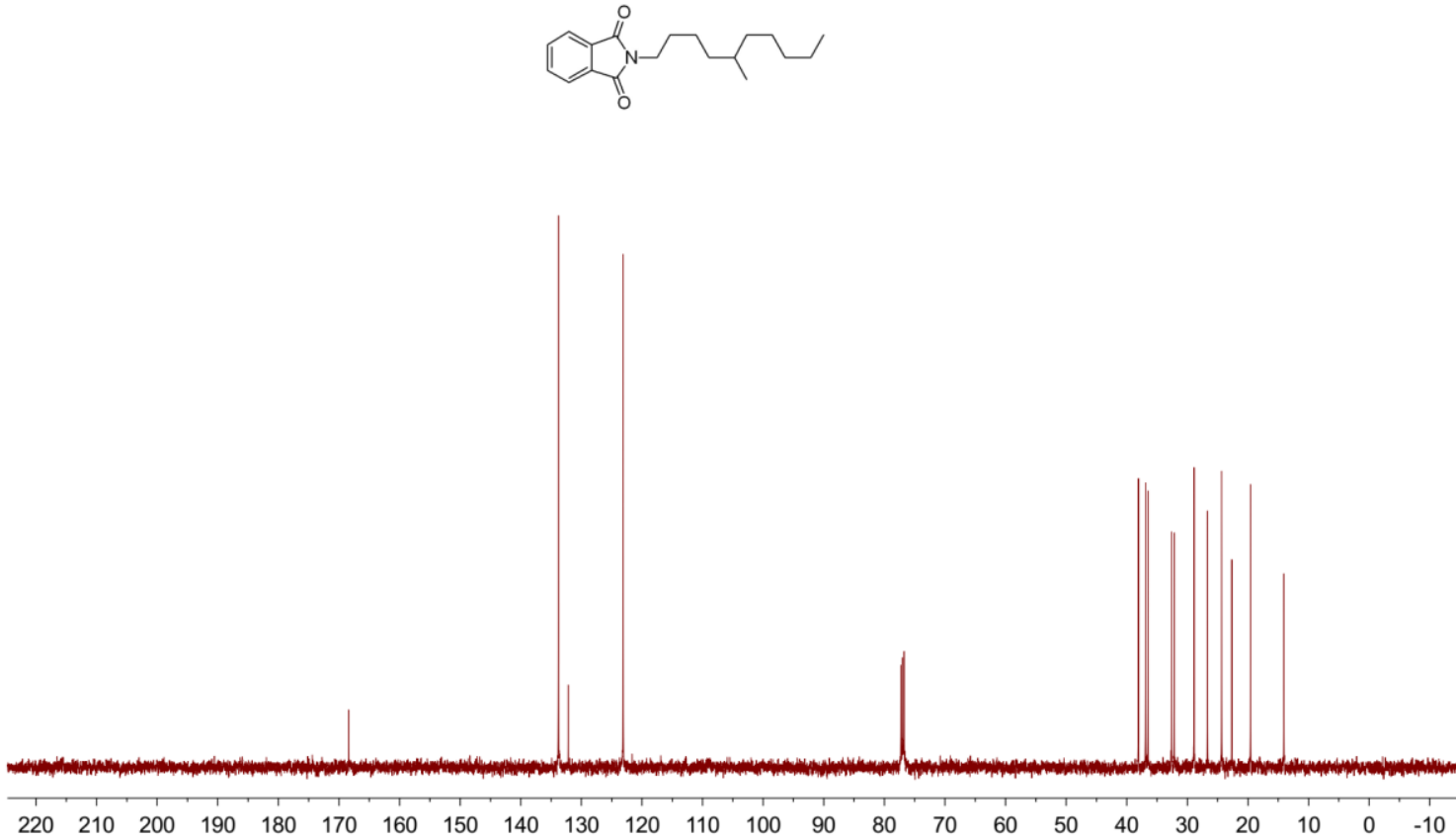
1H NMR (500 MHz, CDG) for 4aq
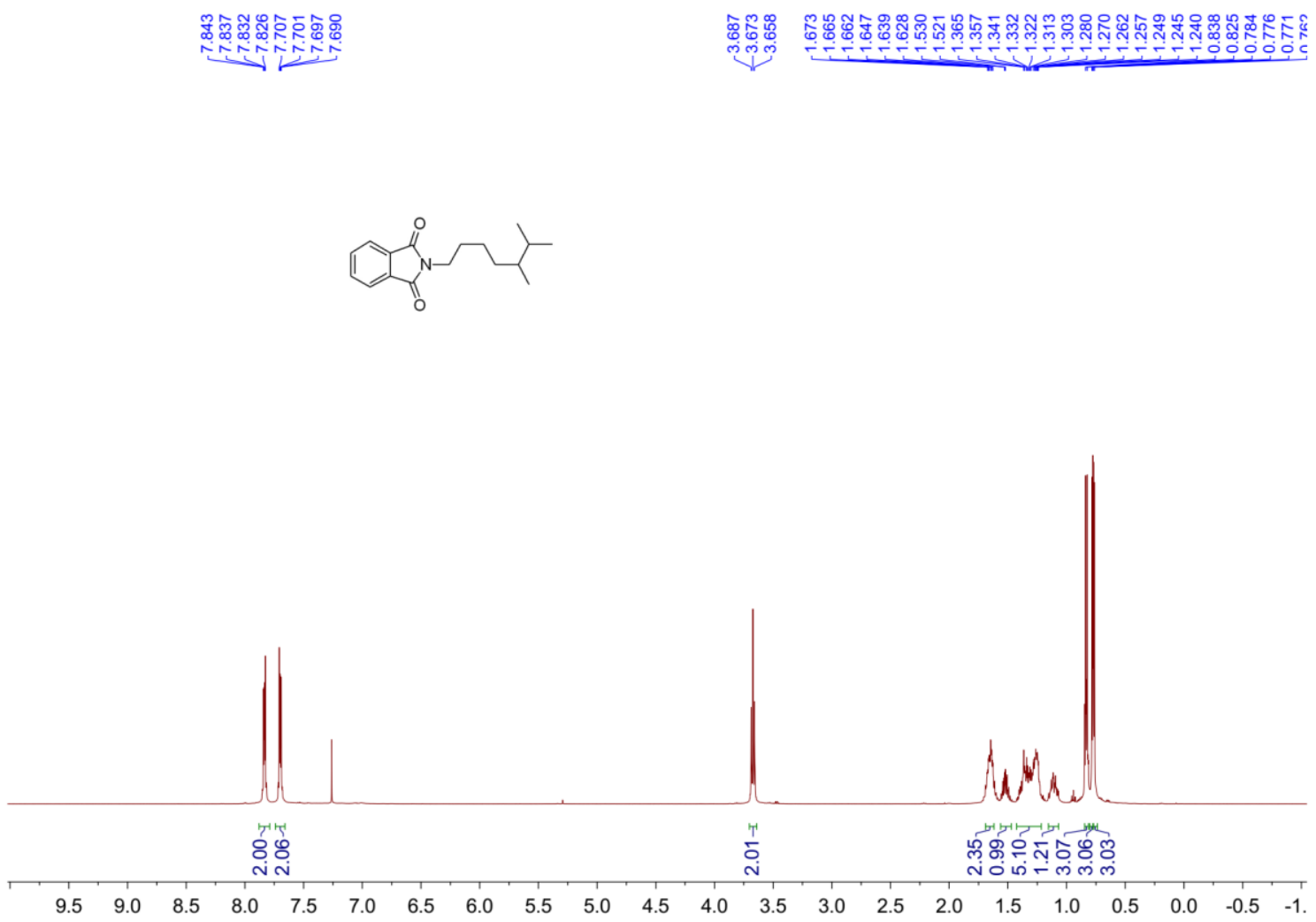

13C NMR (126 MHz, CDG) for 4aq

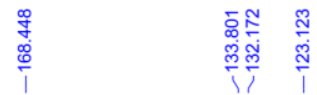

이유ํำ

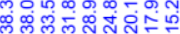
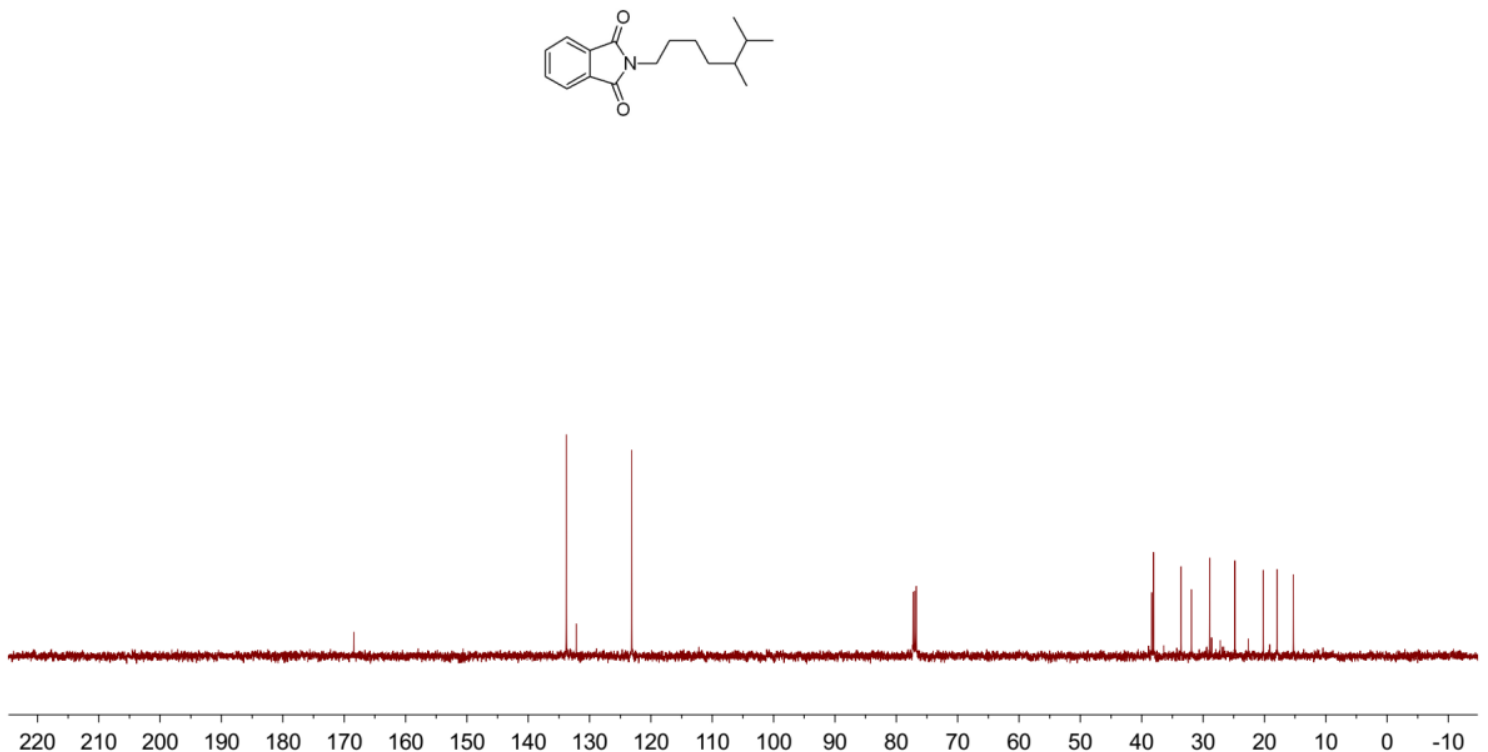
1H NMR (500 MHz, CDG) for 4ar

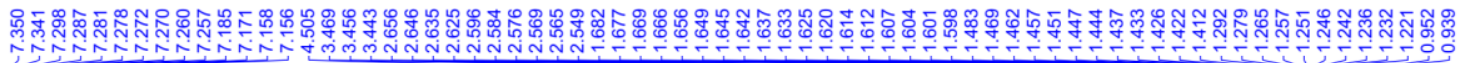
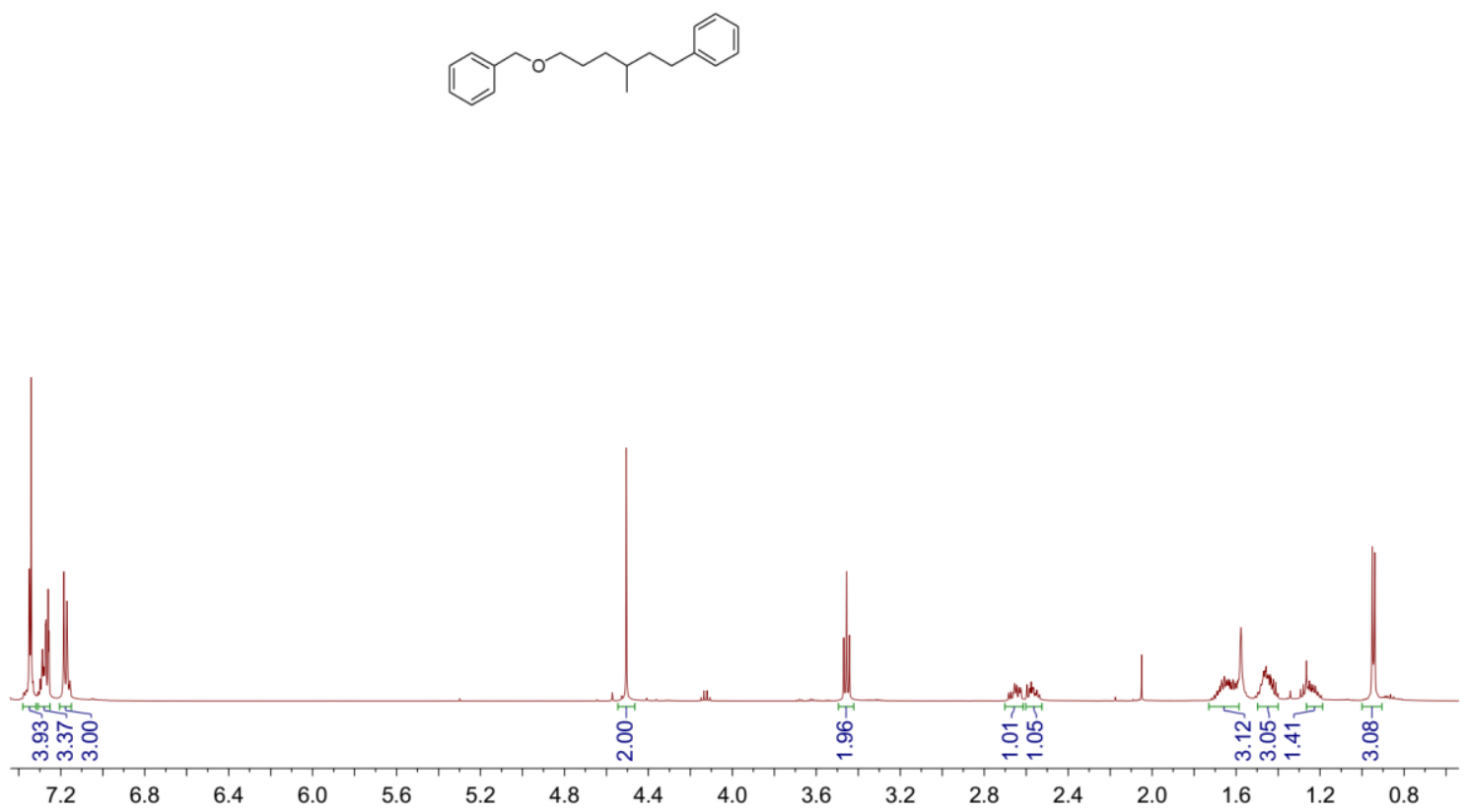

13C NMR (126 MHz, CDG) for 4ar

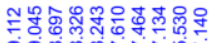

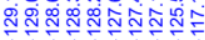

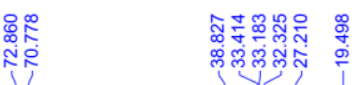
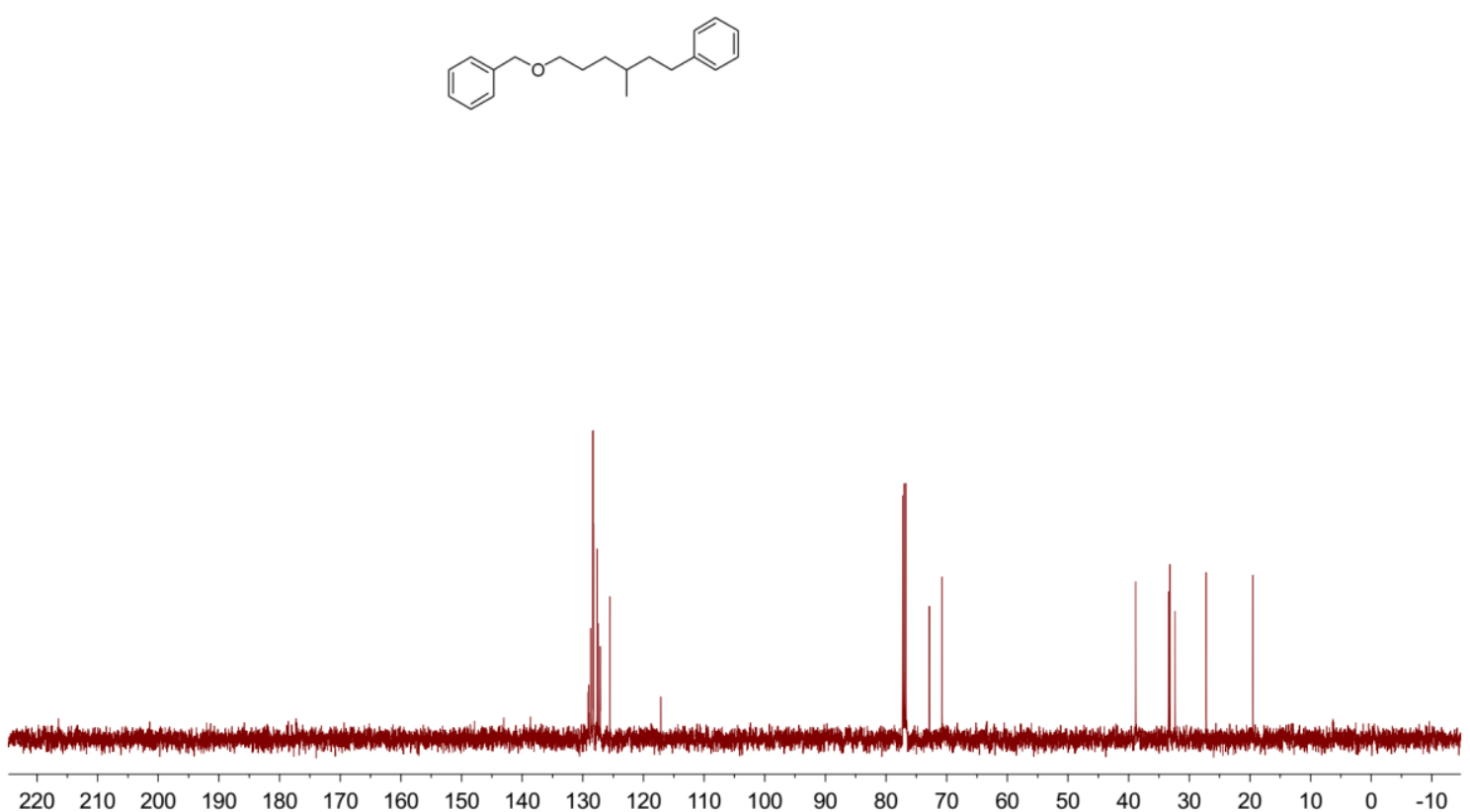
1H NMR (500 MHz, CDG) for 4as

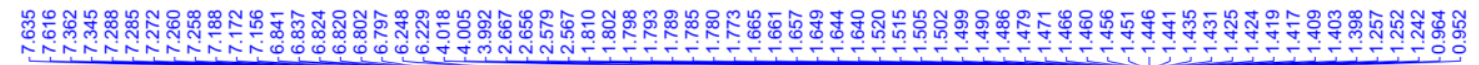<smiles>CC(CCCCOc1ccc2ccc(=O)oc2c1)CCCc1ccccc1</smiles>

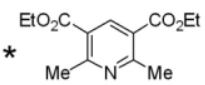

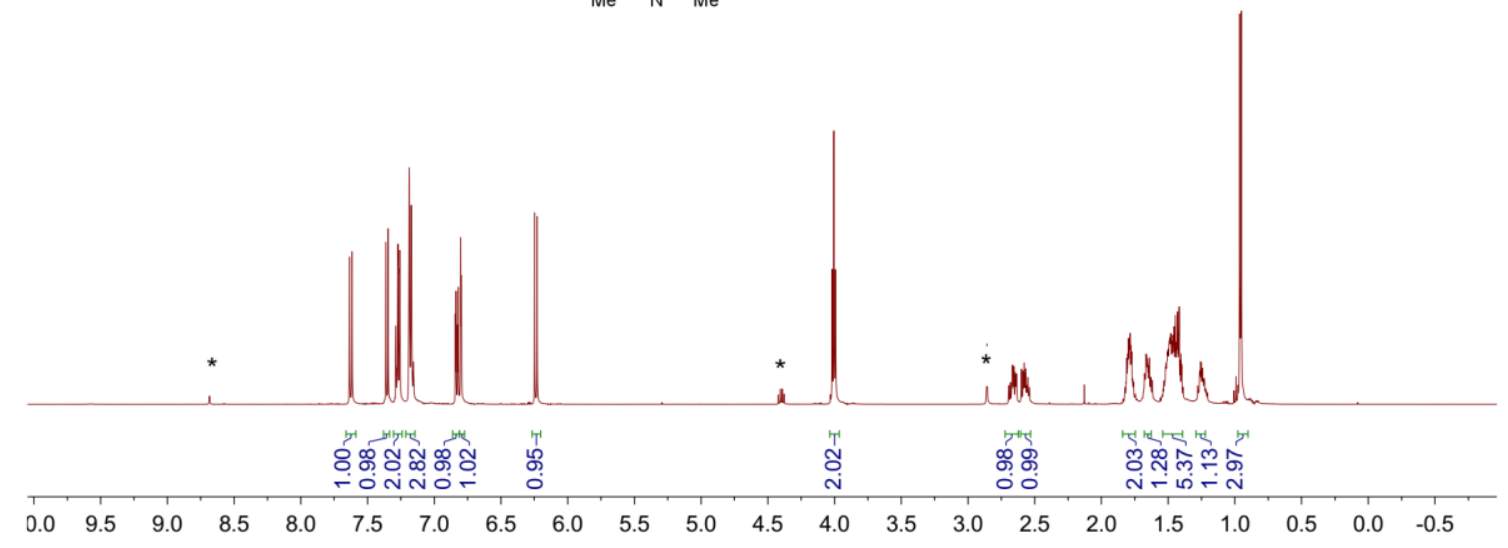

13C NMR (126 MHz, CDG) for 4as

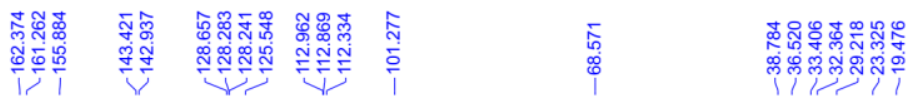
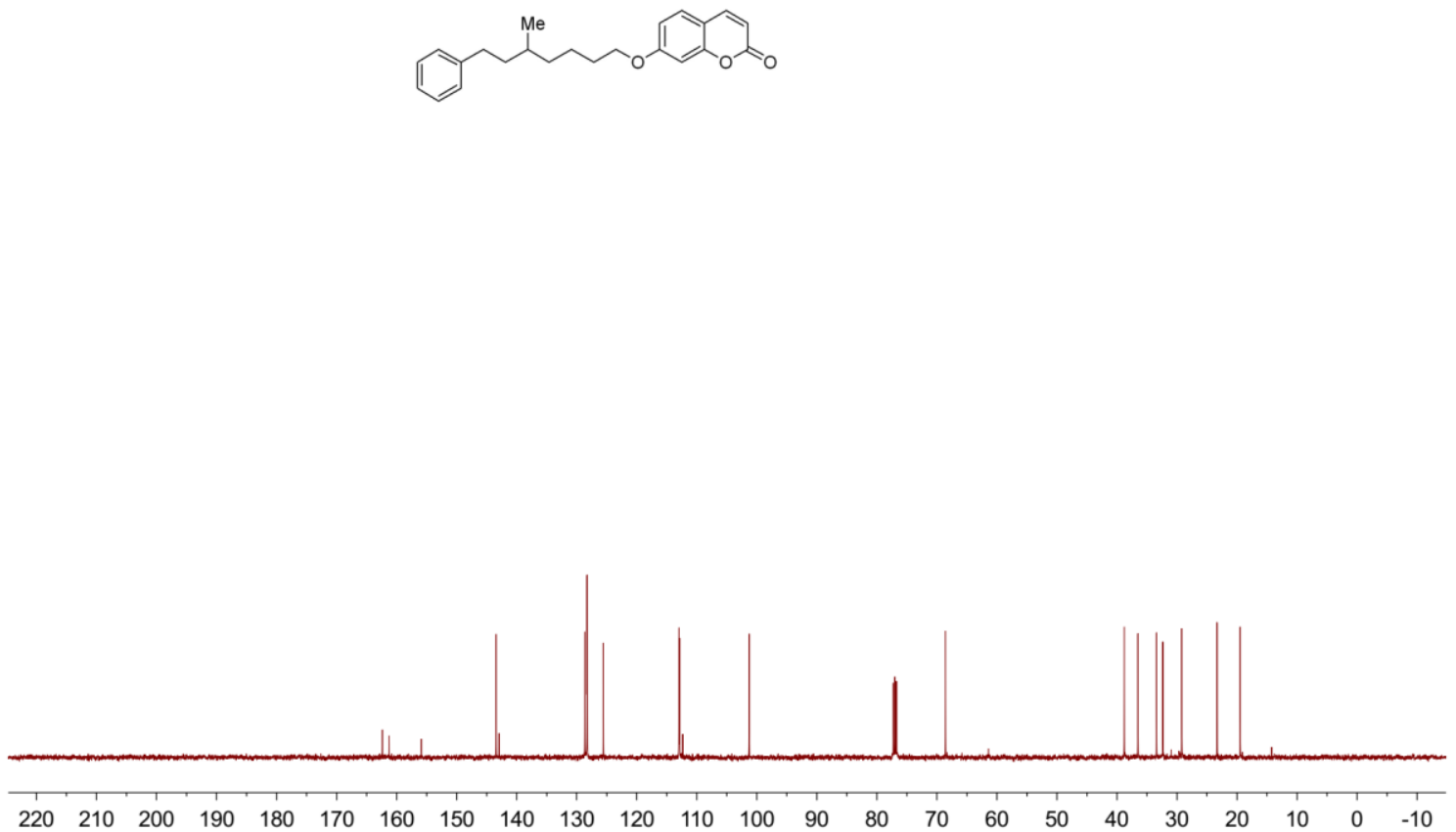
1H NMR (500 MHz, CDG) for 4at

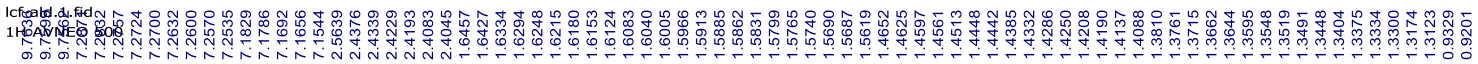

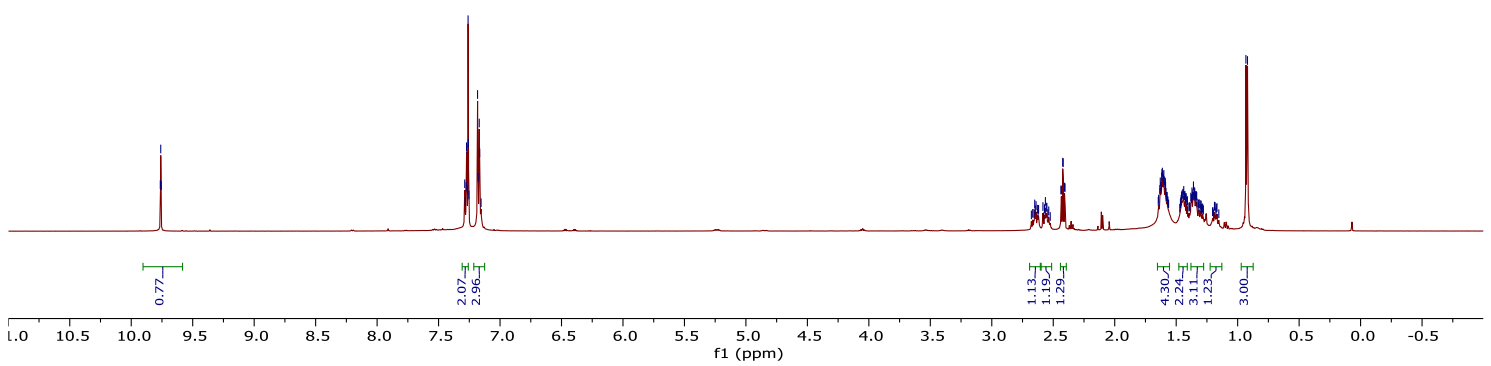

13C NMR (126 MHz, CDG) for 4at

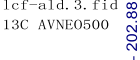



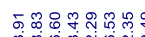

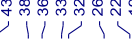

Y

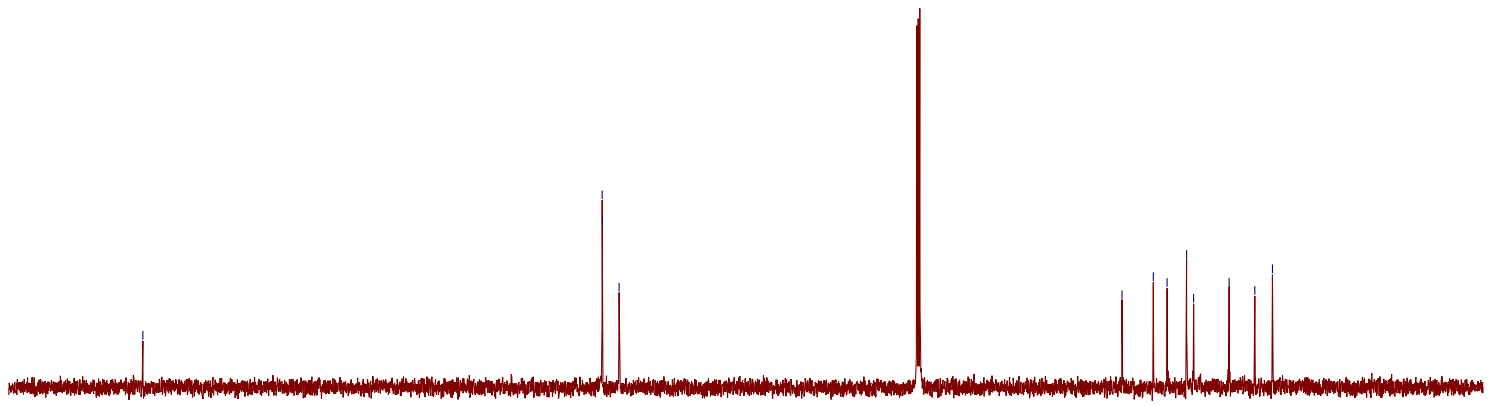

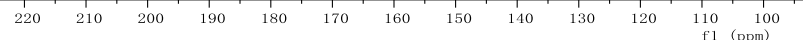


1H NMR (500 MHz, CDG) for 4au

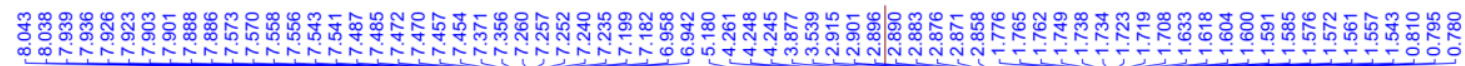

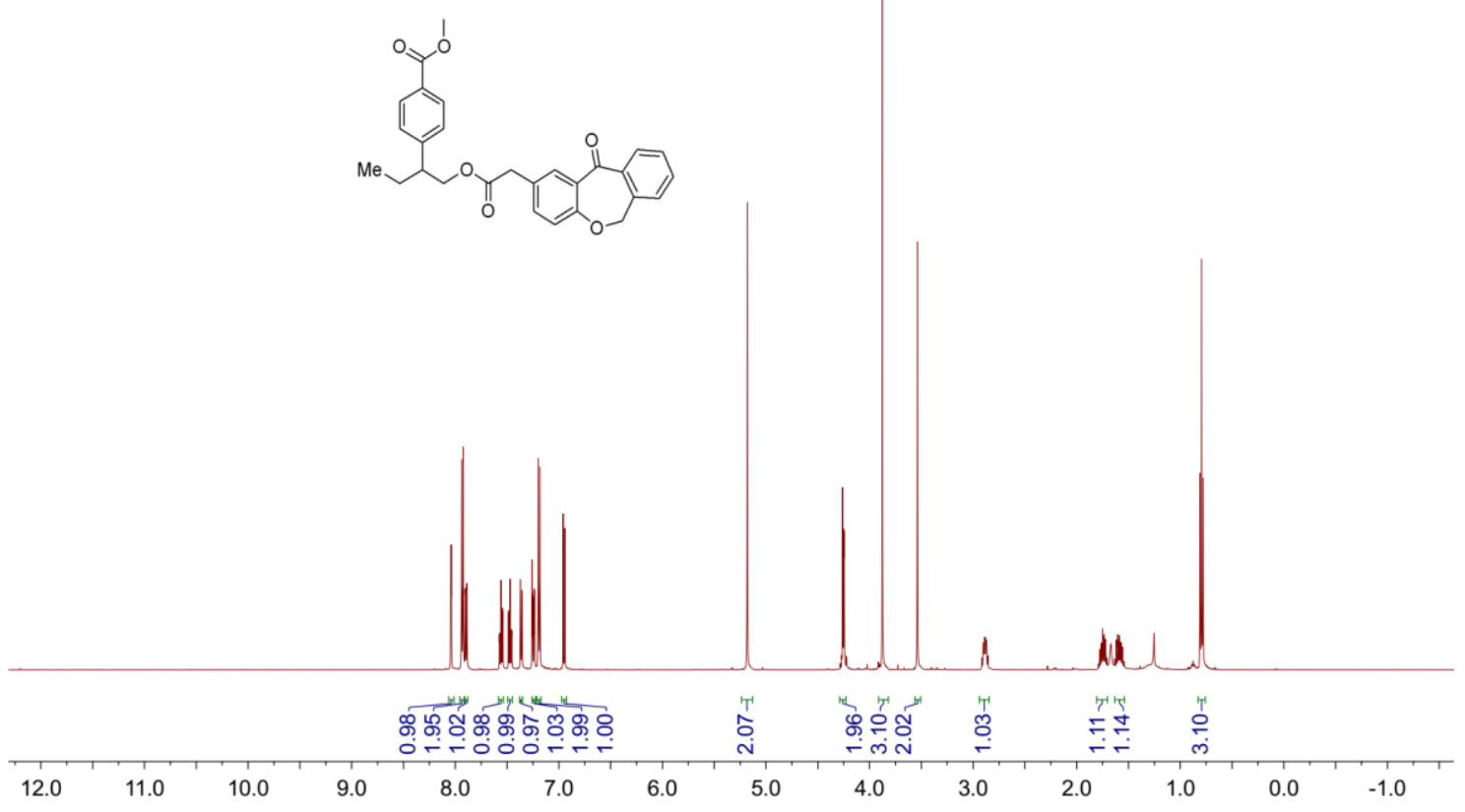

${ }^{13}$ C NMR (126 MHz, CDG) for $4 a u$

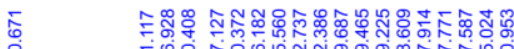

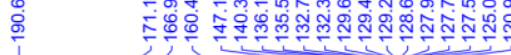

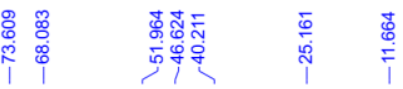
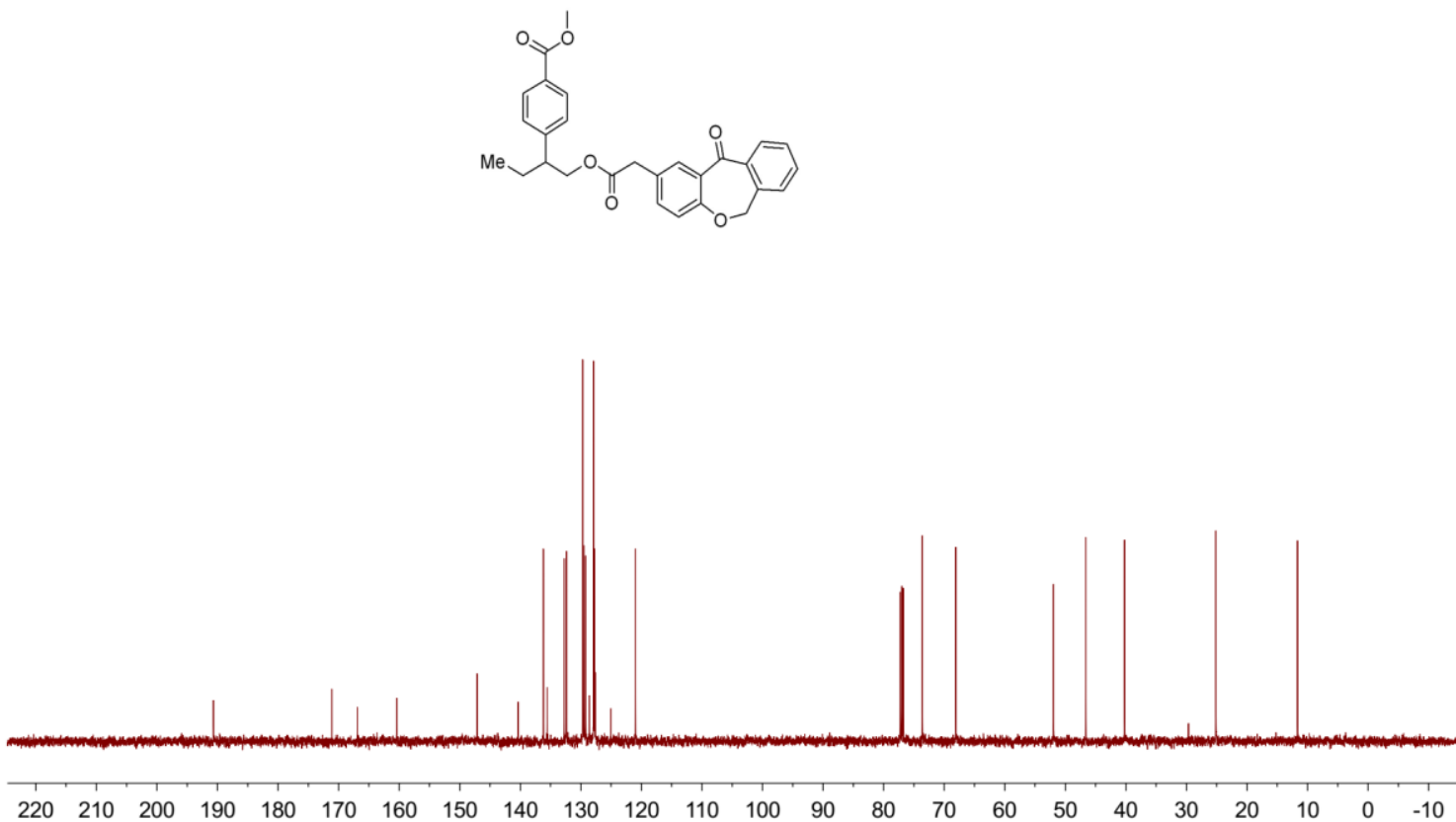
1H NMR (500 MHz, CDG) for 6

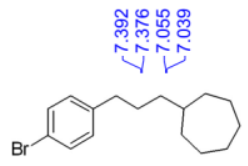

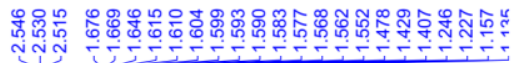

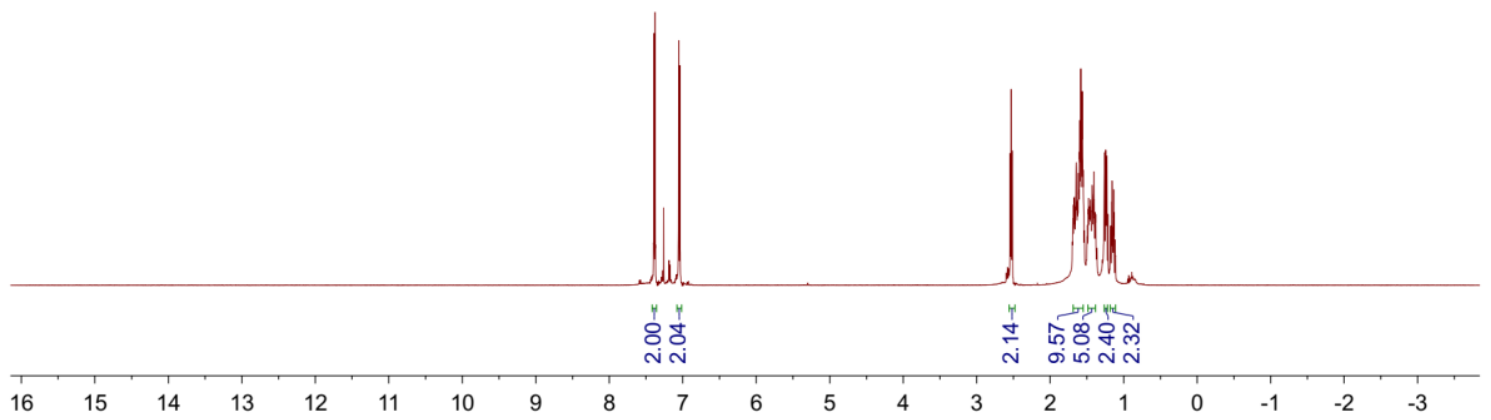

13C NMR (126 MHz, CDG) for 6

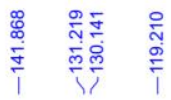

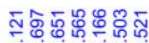

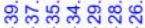<smiles>Brc1ccc(CCCC2CCCCC2)cc1</smiles>

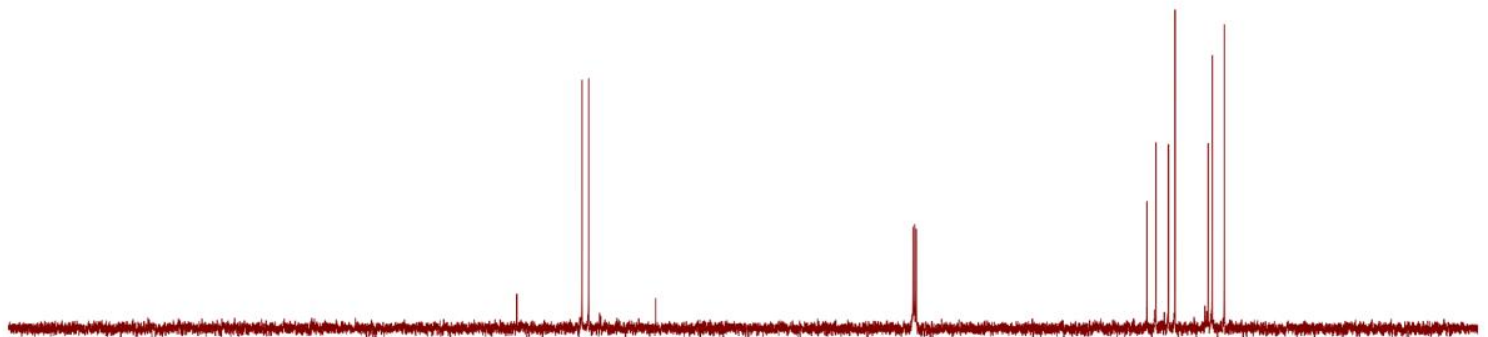

$\begin{array}{llllllllllllllllllllllll}220 & 210 & 200 & 190 & 180 & 170 & 160 & 150 & 140 & 130 & 120 & 110 & 100 & 90 & 80 & 70 & 60 & 50 & 40 & 30 & 20 & 10 & 0 & -10\end{array}$ 
1H NMR (500 MHz, CDG) for 7

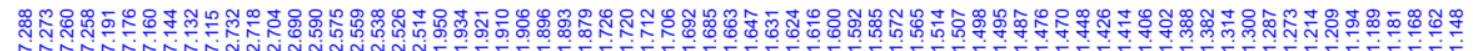

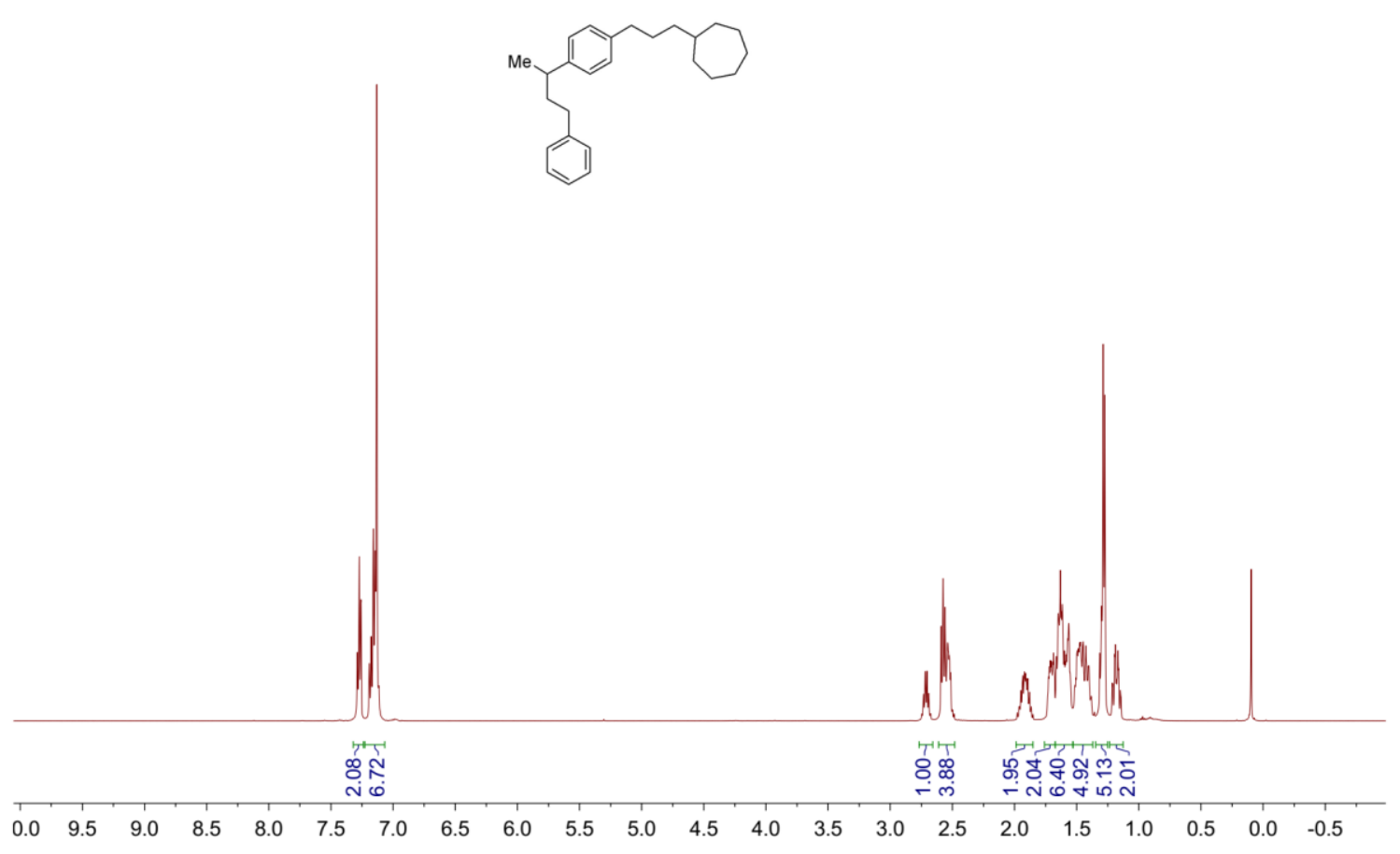

13C NMR (126 MHz, CDG) for 7

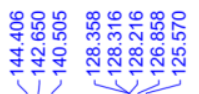

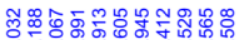

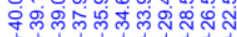
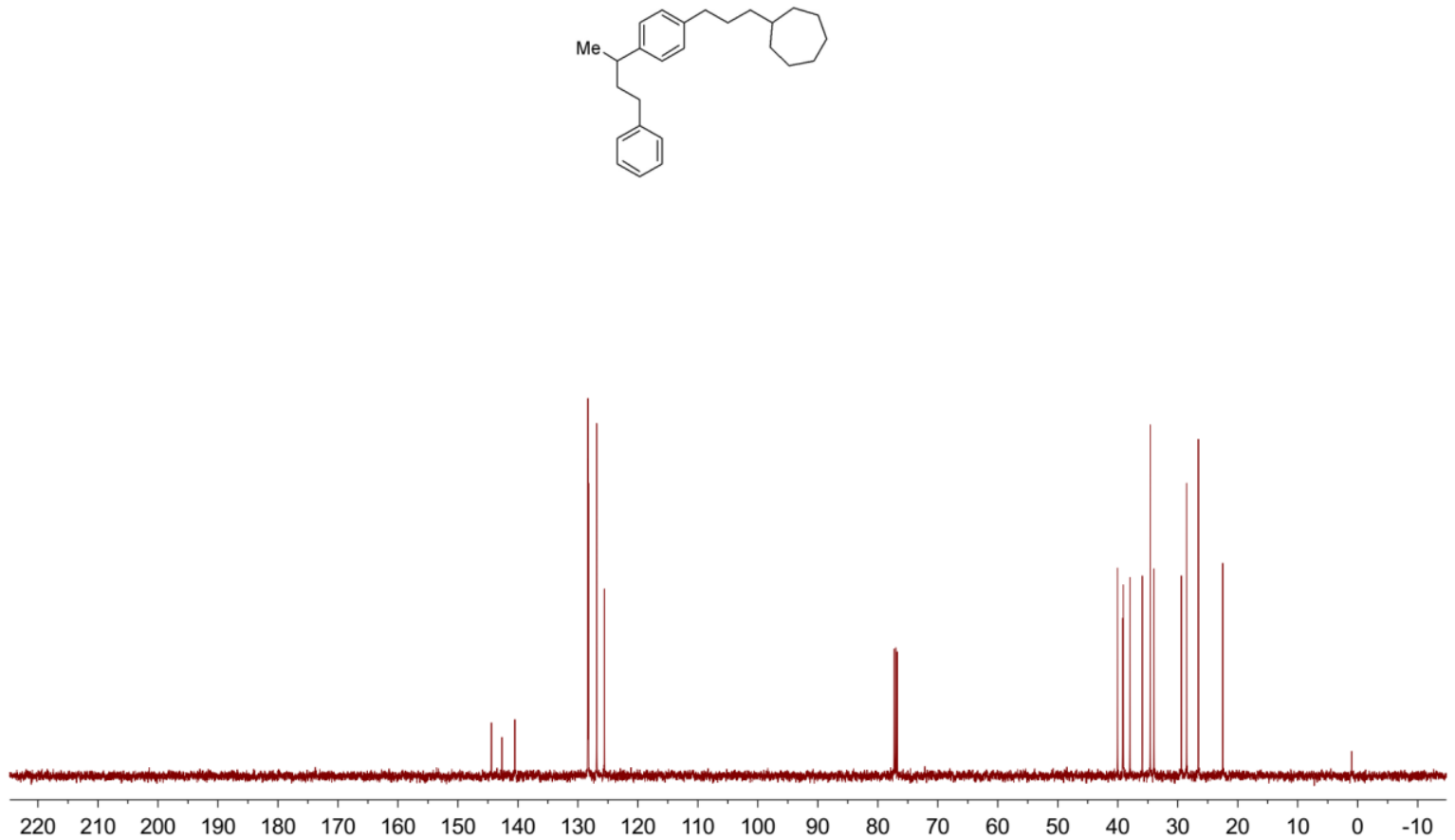
1H NMR (500 MHz, CDG) for 8

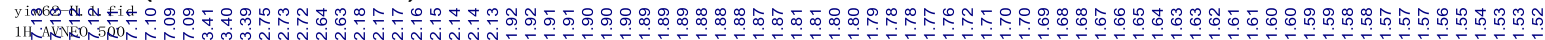

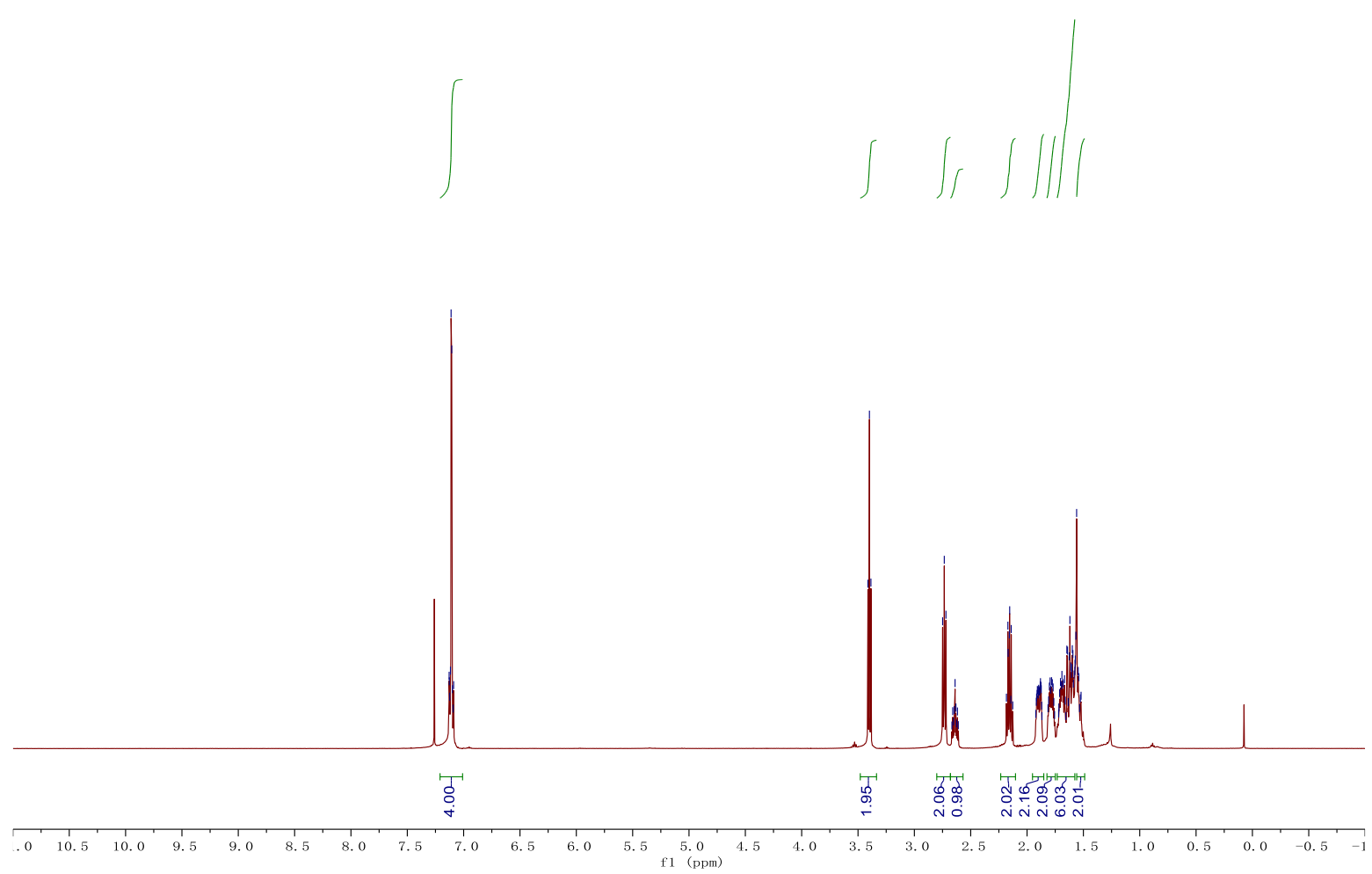

13C NMR (101 MHz, CDG) for 8

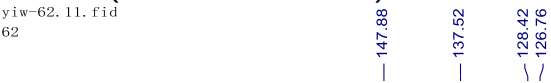

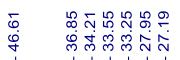

过

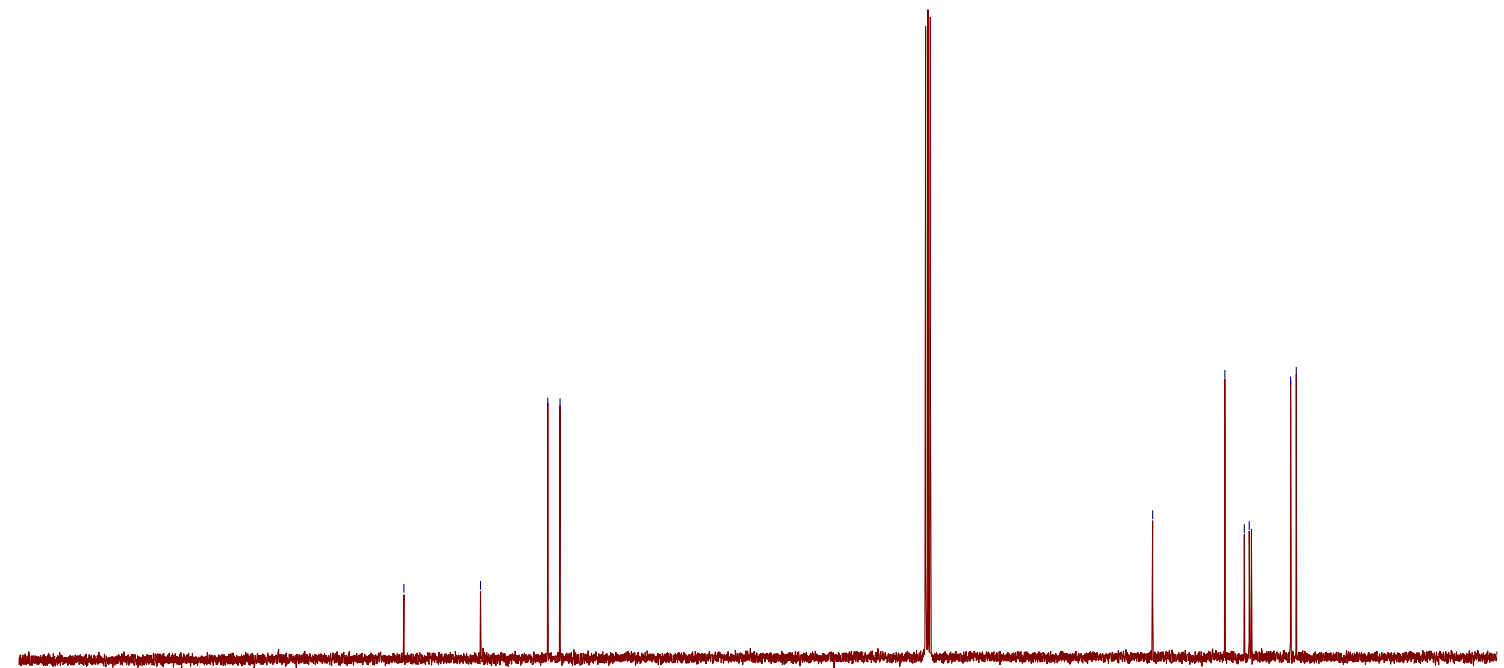

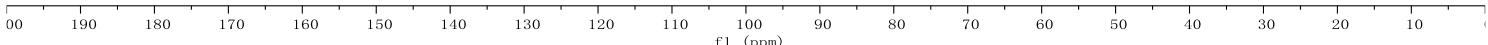


1H NMR (500 MHz, CDG) for 9

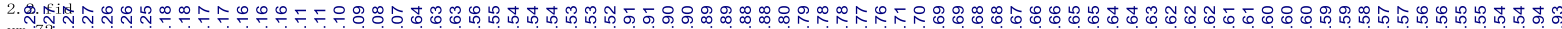

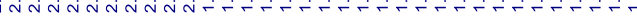

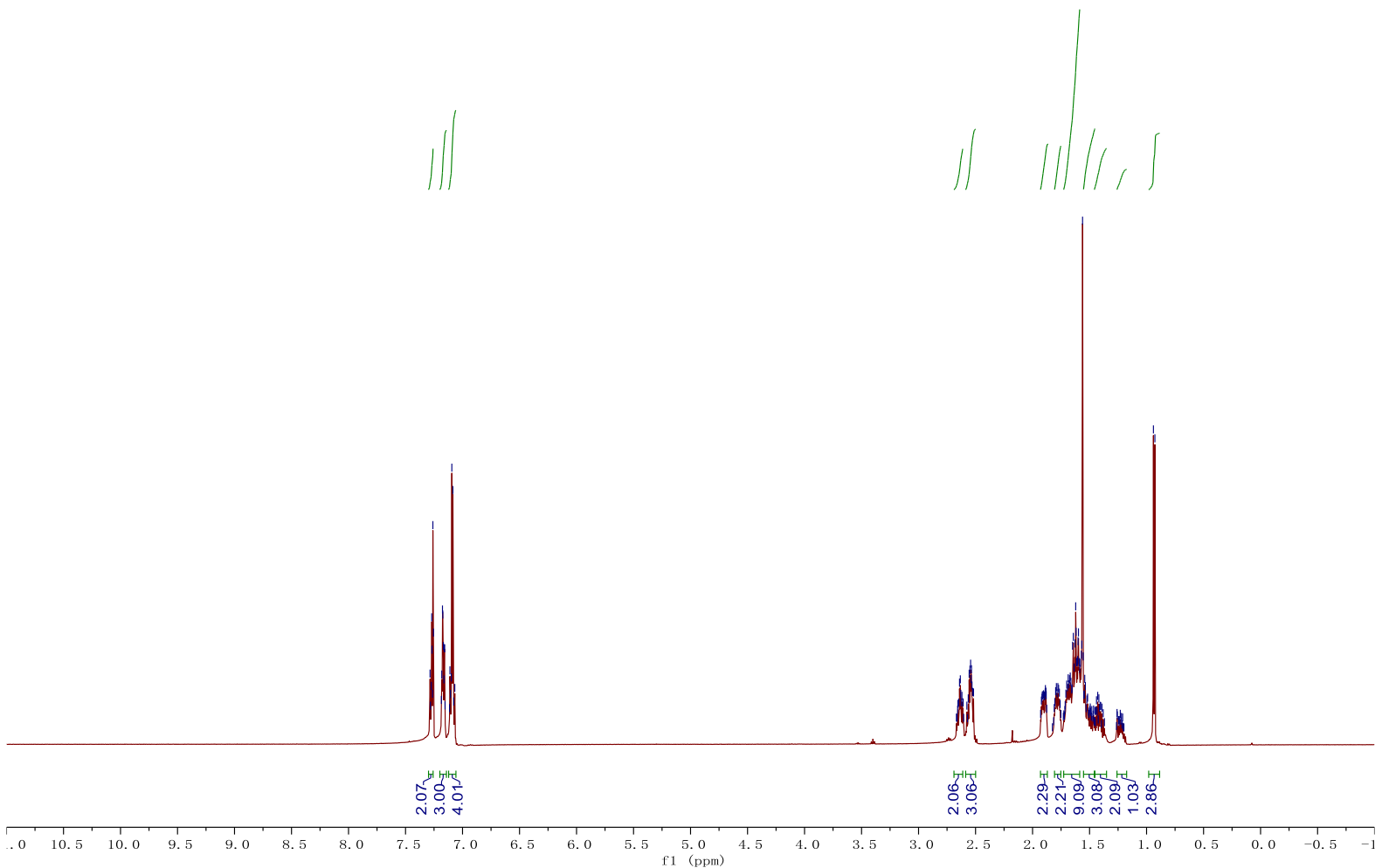

13C NMR (101 MHz, CDG) for 9
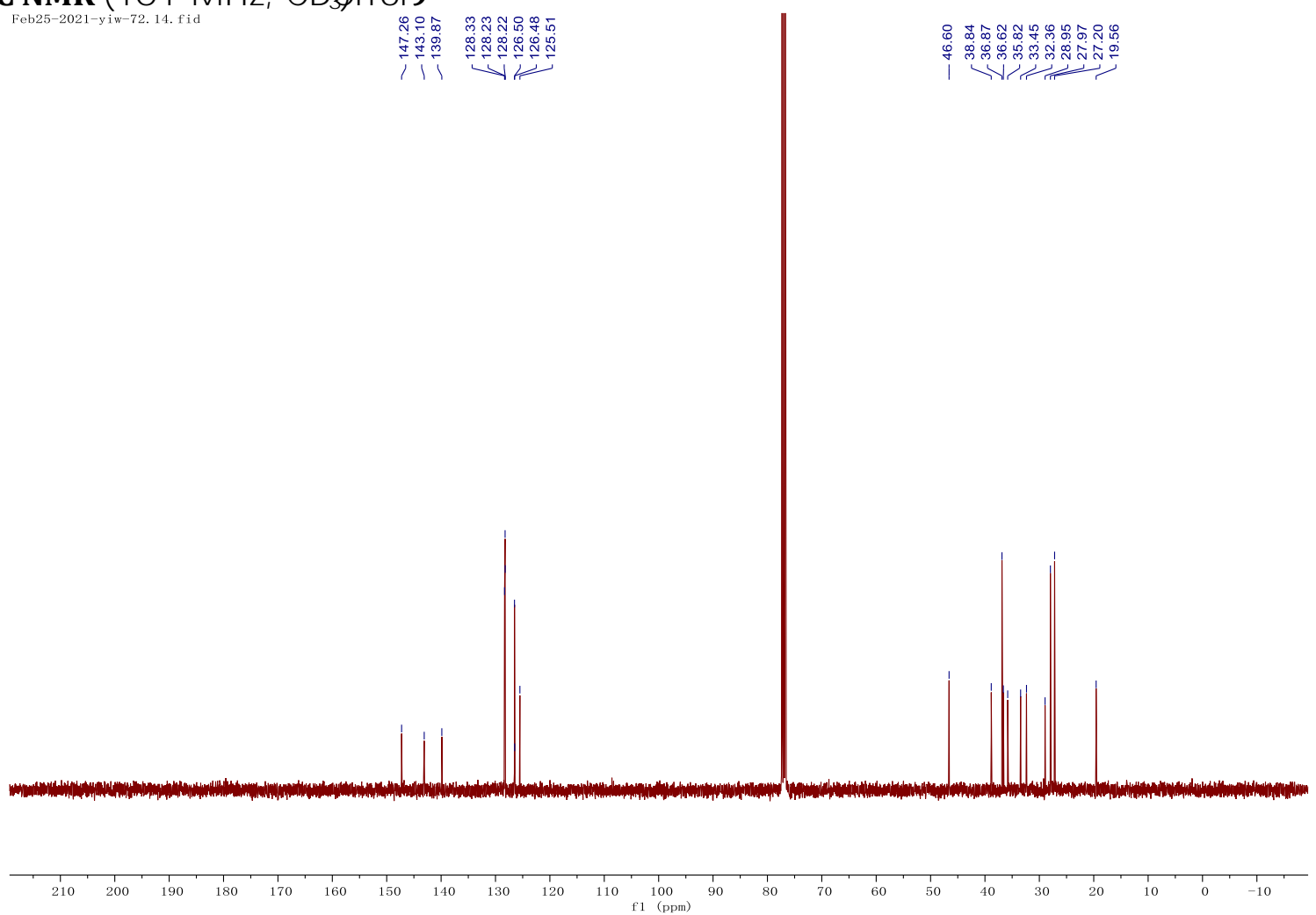
1H NMR (500 MHz, CDCb) for Ni-1

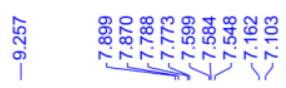

怘
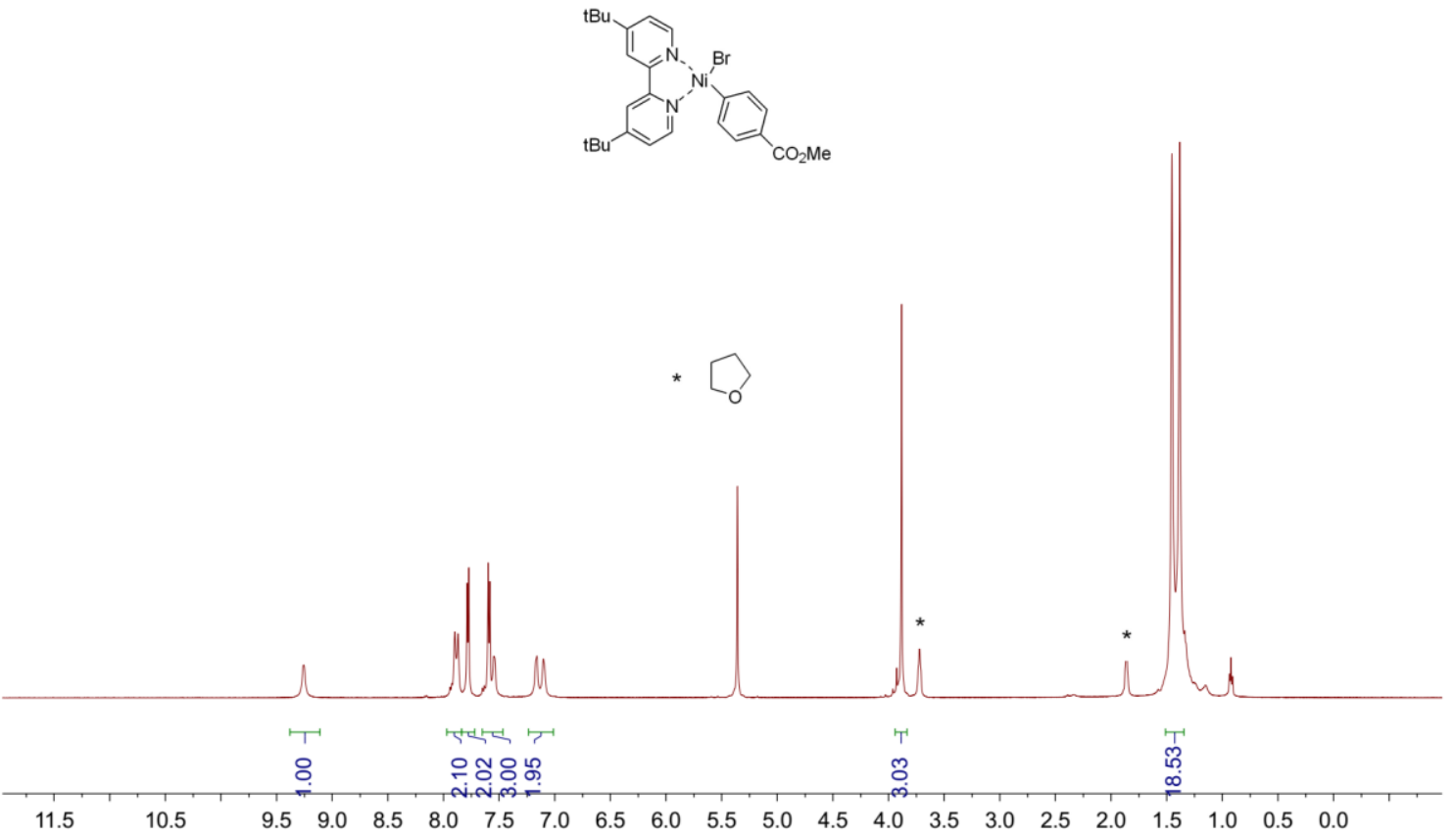